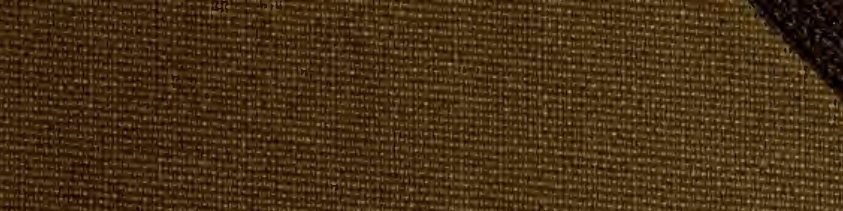

(2)

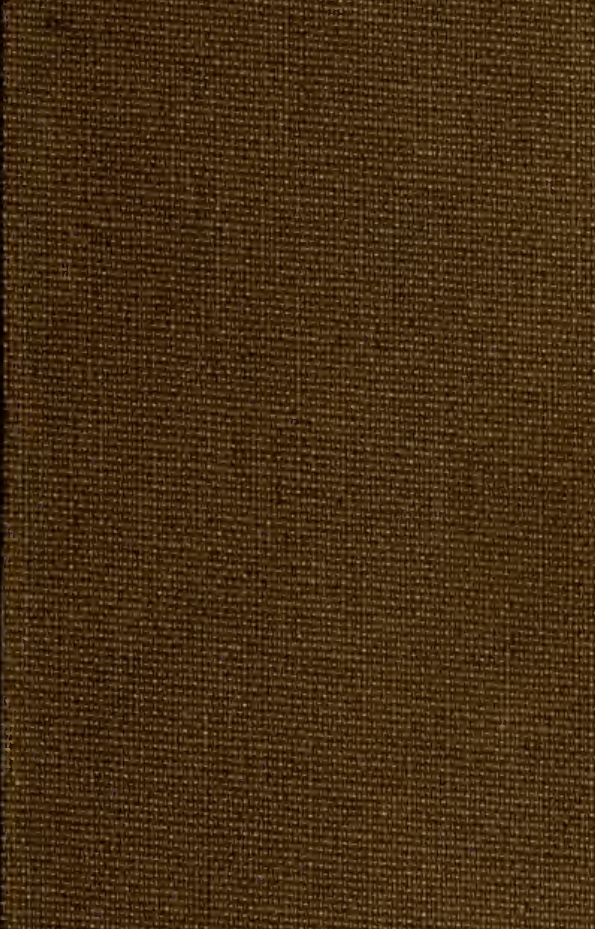

19. 


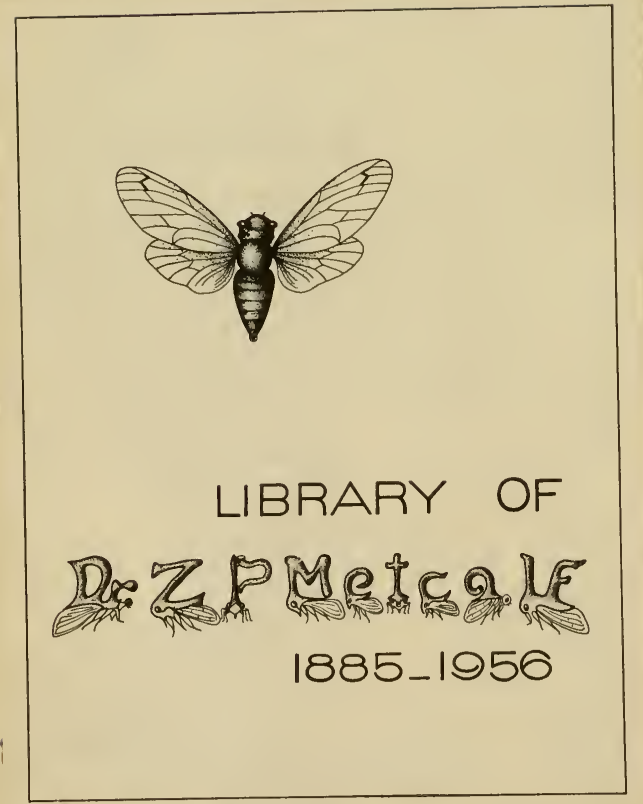








\title{
GLI INSETTI
}

\author{
INTRODUZIONE
}

\section{ALLO STUDIO DELL'ENTOMOLOGIA}

PER

\section{LORENZO GAMERANO $187 \% a: 219$}

DOTTORE IN SCIENZE NATURALI

ASSISTENTE AL R. MUSEO ZOOLOGICO DI TORINO

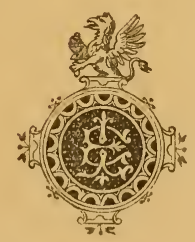

TORINO E ROMA

ERMANNO LOESCHER 
PROPRIETẢ LETTERARIA

Torino. - Stabilimento Tipografico Vincenzo Bona. 
ALLA MEMORIA

DI

\section{VITTORE GHILIANI}

CON AFFETTO DI DISCEPOLO RICONOSCENTE

QUESTO LIBRO

INTITOLA

L'A U TORE. 


\section{PUBBLICAZIONI DELLO STESSO AUTORE}

Polimorfismo nella femmina dell'Hydroplitus piceus. Atti della $R$. Accad. delle Scienze di Torino, vol. XII, 1877.

Considerazioni sul genere Lacerta Linn. e descrizione di due nuove specie. Ati R. Acad. delle Sc. di Torino, vol. XIII, 1877.

Dei caratteri sessuali secondari della Testudo ibera Pallas. Atti R. Accad. delle

Sc. di Torino, vol. XIII, 1877.

Descrizione di una nuova specie del genere Podarcis Wagl. Atti R. Accad. delle Sc. di Torino, vol. XIII, 1878.

Intorno all'anatomia della Nasiterna Pusio Sclat. Atti R. Ac. d.Sc.di Torino, vol. XIII, 1878.

Osservazioni intoruo agli Anfibi anuri del Marocco. Atti $R$. Accad. delle Sc. di Torino, vol. XIII, 1878.

Del Bombice del Salice nel contorno di Torino. Annali della $R$. Accad. d'Agricoltura di Torino, vol. XX, 1877.

Di alcune variazioni avvenute nella fauna entomologica del contorno di Torino. Ann. della R. Accad. d'Agricolt. di Torino, vol. XXI, 1878.

Osservazioni sulla struttura delle elitre di alcuni Coleotteri. Rendic. della Soc. Entomol. Ital., 1877. - Intorno a due Longicorni trovati per la prima volta in Piemonte. Ibid., 1878. - Sopra un caso dubbioso di parassitismo. Ibid., 1878. - Osservazioni intorno alle specie italiane del genere Oryctes. Ibid., 1878.

Descrizione di un nuovo genere e di una nuova specié di Ortottero piemontese. Atti R. Accad. delle Sc. di Torino, vol. XIII, 1878.

Note intorno ai caratteri sessuali secondari di alcuni Coleotteri. Atti R. Accad. delle Sc. di Torino, vol. XIII, 1878.

Descrizione di alcuni insetti mostruosi delle raccolte entomologiche del museo di Torino. Atti R. Accad. delle Sc. di Torino, vol. XIV, 1878.

Descrizione di una nuova specie del genere Chlaenius Bon. Atti R. Accad. delle Sc. di Torino, vol. XIV, 1878.

Osservazioni intorno al Phyllodactylus Doriae. Atti R. Accad. delle Sc. di Torino, vol. XIV, 1878.

Studi intorno alle specie del genere Geniates. Atti $R$. Accad. delle Sc. di Torino, vol. XIV, 1878.

Studi sul genere Discoglossus. Atti R. Acc. delle Sc. di Torino, vol. XIV, 1879.

Di alcune specie di Anfibi anuri esistenti uelle collezioni del R. Museo Zoologico di Torino, Atti R. Accad. delle Sc. di Torino, vol. XIV, 1879.

Osservazioni intorno ai caratteri sessuali secondari dell'Anguis fragitis. Atti $R$. Accad. delle Sc. di Torino, vol. XIV, 1879.

Ricerche intorno alla struttura dei peli-ventose dei tarsi dei Coleotteri. Atti $\boldsymbol{R}$. Accad. delle Sc. di Torino, vol. XIV, 1879. 
degli Insetti :

Chi non e preoccupato di presiudici, non cessa di inoltrarsi per essi in cognizioni della Natura; apprende dall'oprar loro la forza e l'indole dei di lei stromenti nella materia soggetta: da che trovandoli, or in terra, or in piante, or in sassi, fin nei durissimi scorli love penetrano a forza di lime invisibili, ove lasciarono impresse sempre vestigia d'arteficj maggiori della coinune meccanica) approfitta di tante notizie, che la lor minor parte prevale a quanto per loro studio ne trassero i noti Filosofi tutti insieme dagli animali grandi. Altrettanto $\mathrm{s}$ 'ha dalla notomia d'ouni insetto visibile tanto da poter anatomizzarsi : da qualunque loro sezione si cava una comparazione ed un progresso d'intendimento per quella de' grandi.

VALLISNIERI, Op. fis. med.. vol. I, p. 308 .

I Professori di Storia Naturale che ogni anno insegnano tutta la scienza, gli autori dei trattati scolastici e in generale quelli che non fanno altro che occuparsi della Storia Naturale, sogliono mostrare un certo disprezzo per quegli studiosi che essi chiamano dilettanti; vale a dire quei tali che mentre hanno per scopo principale della vita altre occupazioni, consacrano una parte del loro tempo ad un ramo limitato della Storia Naturale occupandosi esclusivamente di esso.

Questi cultori parziali della scienza sono molto benemeriti e meritano gratitudine e non disprezzo. Questi cultori sono per buona ventura molto numerosi presso le nazioni meglio incivilite.

Il Bollettino della Società Entomologica di Francia nella lista dei suoi membri ha militari, magistrati, ecclesiastici, commercianti, proprietari di poderi, maestri elementari e impiegati di ogni amministrazione. Senza essere naturalisti di professione, questi cosidetti dilettanti di entomologia rendono, ripeto, servizii segnalati alla scienza. 
La vita degli insetti è stata certamente molto studiata: ma questi piccoli esseri sono cosi maravigliosamente vari, gli atti della loro vita sono tanto diversi e i loro rapporti cogli altri animali e coll'uomo nella vita sociale sono così molteplici, che quello che ancora si ignora intorno ad essi è senza paragone più di quello che si sa.

I grandi concetti che oggi occupano le menti e promuovono le discussioni più ardenti e promettono un nuovo aspetto alle scienze, hanno il loro fondamento nella minuta osservazione dei costumi e delle forme. Ora la classe degli insetti è, fra tutte le altre del regno animale, quella che ha fornito e che fornisce tuttora maggiori elementi alla discussione.

Per la qualcosa non sarà mai sufficiente la gratitudine della scienza per quei suoi modesti cultori che pur non consacrando ad essa la parte principale della loro vita, cosi efficacemente tuttavia ne accrescono e consolidano l'edifizio.

Nessuno oggi pone più in dubbio la verità di questo asserto che - una nazione tanto può quanto sa - E per sapere di una nazione s'intende la maggior possibile diffusione del maggior numero di cognizioni in tutte le classi sociali, perchè oggi non basta più ad una nazione una grande coltura limitata ad una classe o ad un sol ramo del sapere.

Come conseguenza diretta di cio deriva il fatto che le nazioni più potenti del mondo sono oggi quelle presso le quali meglio si coltivano le scienze naturali e i vari rami di esse.

L'Italia fino ad oggi nell'entomologia ebbe qua e colà qualche cultore segnalatissimo, ma quei cultori parziali, quei dilettanti, di cui ho detto sopra, cosi numerosi segnatamente in Francia, sono in Italia piuttosto rari.

Ora è venuto il tempo, senza dubbio, in cui anche per questo rispetto l'Italia si spingerà avanti nella buona via. Perciò ho creduto opportuno di adoperarmi in questo senso secondo le mie forze e la mia cerchia di azione. 
Nello scorso inverno ho fatto un corso di entomologia agli studenti dell'Università di Torino coll'intendimento di agevolare loro quelle cognizioni fondamentali intorno agli insetti da cui riesce poi piacevole il muovere ad ulteriori ricerche ed osservazioni, ed ebbi la soddisfazione di vedere che non solo corrisposero gli studenti, ma anche in parte il pubblico che si mostrò voglioso di siffatti ammaestramenti.

Lo scopo che mi mosse a fare quell'insegnamento è il medesimo che mi spinge a pubblicare questo volume col quale io intendo di spianare la via ai principianti e segnare alcune norme generali per lo studio dell'entomologia.

Ho fatto vedere il manoscritto a parecchi colleghi ed amici e tutti me lo hanno criticato. Il biasimo principale mi venne da ciò che, secondo che mi dicono, mi sono esteso troppo intorno all'anatomia degli insetti. Non bisogna confondere, mi si dice, l'anatomia colla zoologia; lo studio diligente delle forme esterne e dei costumi basta largamente a soddisfare le esigenze più rigorose di una buona classificazione; l'anatomia è un altro studio. Io non credo che ciò si possa più oggi sostenere. Lo studio delle forme esterne non vivificato da quello dell'interna struttura non ha significato; si vedono le parti, ma non si comprende il perchè di esse; mentre lo studio della struttura rivela gli uffici e la ragione d'essere di ciò che si vede di fuori. Lo studio dell'anatomia è inoltre necessario all'intelligenza dei costumi degli insetti, per cui se io ho un rincrescimento si è piuttosto di aver fatto poco che non troppo in questa via.

Mi si parla pure delle sovverchie difficoltà che presentano le ricerche anatomiche; queste ricerche richiedono certamente un po' più d'attenzione, ma il mettere allo scoperto il canal digerente o i centri nervosi di un insetto non è cosa che superi le facoltà intellettuali di nessuno e la soddisfazione che si prova da queste ricerche è tale che molto più tenacemente invita il principiante a continuare. 
Mi fu fatto pure il rimprovero di non aver sviluppato in pari grado la trattazione dei vari ordini degli insetti. La ragione di ciò si è questa che certi ordini di insetti sono assai meno e altri assai più noti. Io mi sono esteso di più intorno ai primi, meno invece intorno ai secondi.

Questi sono gli appunti che mi vennero fatti prima ancora che il libro sia pubblicato; desidero ardentemente che me ne vengano fatti altri in molto più gran numero dopo la pubblicazione, perchè essi mi proveranno che il libro avrà trovato lettori e mi ammaestreranno per lo avvenire.

Torino, giugno 1879.

Lorenzo Camerano. 


\section{CENNO STORICO}

INTORNO ALLA ENTOMOLOGIA

Nell'anno 1668 pubblicavasi in Firenze un libro ornato di belle tavole, e contenente la relazione di varie esperienze intorno alla generazione degli insetti. Autore di questo libro (1) era Francesco Redi, celebre medico e non meno celebre poeta d'Arezzo.

Fra gli esperimenti dal Redi descritti nel libro testè menzionato, due, i più semplici e concludenti, che divennero in breve famosi, mi piace di riferire servendomi delle parole stesse del Redi, imperocchè queste cose non si potrebbero esporre in modo più chiaro e con stile più leggiadro.

"Io cominciai a dubitare, se per fortuna tutti i bachi delle carni dal seme delle sole mosche derivassero, e non dalle carni stesse imputridite, e tanto più mi confermava nel mio dubbio, quanto che in tutte le generazioni da me fatte nascere, sempre avea io veduto sulle carni, avanti che inverminassero, posarsi mosche della stessa spezie di quelle, che poscia ne nacquero; ma vano sarebbe stato il dubbio, se l'esperienza confermato non l'avesse. Imperciocchè, a mezzo il mese di luglio, in quattro fiaschi di bocca larga misi una serpe, alcuni pesci di fiume, quattro anguillette d'Arno, ed un taglio di vitella di latte; e poscia, serrate benissimo le bocche con carta e spago, e benissimo sigillate, in altrettanti fiaschi posi altrettante delle suddette cose, e lasciai le bocche aperte; nè molto passò di tempo che i pesci

(1) Esperienze intorno alla generazione degli insetti fatte dal sig. Francesco Redi e da lui scritte in una lettera al sig. Carlo Dati. Firenze, 1668. 
e le carni di questi secondi vasi diventarono verminose; ed in essi vasi vedevansi entrare, ed uscir le mosche a lor voglia; ma ne' faschi serrati non ho mai veduto nascere un baco, ancorchè siano scorsi molti mesi dal giorno, che in essi quei cadaveri furono serrati; si trovava però qualche volta per di fuora sul foglio qualche cacchione, o vermicciuolo, che con ogni sforzo e sollecitudine s'ingegnava di trovar qualche gretola da poter entrare per nutricarsi in quei fiaschi, dentro ai quali di già tutte le cose messevi erano puzzolenti, infracidate e corrotte ».

Gli esperimenti che il Redi fece intorno alla generazione degli insetti sono, come appare da quanto ho riferito, semplicissimi ed assolutamente concludenti; tanto semplici anzi, che non possiamo pensare senza meraviglia alla guerra che ebbero a sostenere da parte dei dotti per un lungo periodo di tempo. La guerra che si fece al Redi fu accanita, ed i suoi avversari, non avendo fatti certi, non avendo buone ragioni da contrapporre alle prove del Redi, ricorsero, come al solito avviene in simili casi, agli insulti. Lo si disse eretico, sovvertitore delle leggi, della morale e delle tradizioni sacre. La ragione di questa grande opposizione sta in ció che gli esperimenti del Redi venivano a scalzare, anzi a distruggere in massima parte la teoria allora predominante della generazione spontanea. Quando non si sapeva il modo d'origine di una datá sorta di animali si diceva senz' altro che essi si originavano spontaneamente dalle sostanze in cui gli animali solevano trovarsi. Cosi, ad esempio, la putredine della carne e del formaggio generavano vermi o, come dice elegantemente il Redi, bacavano. Era cosi comoda la teoria della generazione spontanea che sapeva male agli oppositori del Redi di abbandonarla e di rivolgersi alla fatica dell'osservazione e dello sperimentare.

Il Redi del resto aveva preveduto la lotta, ed aveva fatto stampare come epigrafe al suo libro un proverbio arabo cosi concepito: "Chi fa delle esperienze accresce il sapere. Chi è credulo aumenta l'errore ».

Gli esperimenti del Redi ebbero un'importanza capitale per la scienza. Ripetuti da altri in Italia, da Antonio Leuwenhoeck e da Giovanni Swammerdam in Olanda, furono il principio di una serie d'investigazioni intorno alla vita ed intorno alla strut- 
tura degl'insetti, dalle quali ebbe origine un nuovo ramo dello studio degli animali: l'entomologia.

Non si creda tuttavia che l'entomologia, o lo studio degli insetti, abbia cominciato solamente al tempo del Redi. Lo studio degli insetti, che dopo il Redi venne ridotto a scienza, cominciò, molto probabilmente, nello stesso modo in cui ebbe origine lo studio degli altri animali. L'uomo da principio, senza dubbio, divise gli animali, e quindi anche gli insetti, in due categorie: in animali che gli erano nocevoli ed in animali che gli erano utili, e cercó di difendersi dai primi e di trarre il maggiore giovamento possibile dagli altri.

Secondo gli storici Cinesi, la coltura del filugello sarebbe stata introdotta in Cina da uno dei primi monarchi, e risalirebbe a tempi anteriori a quelli di Omero (1). Leggesi pure che Mosè promette agli Ebrei di condurli in una terra ove scorre il latte ed il miele.

La religione contribui pure moltissimo a far rivolgere l'attenzione degli uomini agl'insetti.

I ministri di una religione molto antica, il Sabeismo, credettero di aver trovato nei costumi di certi insetti molto comuni, e che colpivano l'immaginazione della gente, immagini atte ad esprimere alcune idee del loro culto, e ne fecero oggetto di emblemi e di allegorie. Fra gli Egizii uno degl'insetti, ad esempio, che godevano la più alta stima e venerazione, era lo scarabeo sacro. Questo insetto, per alcune particolaritá della sua struttura e dei suoi costumi, aveva fatto rivolgere a sè l'attenzione degli Egiziani. Gli Egiziani lo consideravano come il messaggiero della primavera, e la sua riproduzione ed i suoi

(1) La coltura del filugello ha avuto origine in tempi antichissimi. Trovasi infatti nel Chow-king menzione dell'allevamento del filugello sotto il regno di Zao, 2200 anni avanti l'êra volgare. Il signor S-t Julien (Comptes rendus de T. Académie des Sciences, t. XXIV, p. 1071, 1847) fa risalire l'origine della sericoltura a tempi anche più antichi.

Oltre la Cina, una gran parte dell'Asia coltiva pure, da tempi antichissimi, il filugello. Si fu appunto dalla Persia, verso il decimo secolo, che venne introdotto a Costantinopoli, di dove poi si estese a quasi tutta l'Europa centrale e meridionale.

Verso il quattordicesimo secolo, venne pure la coltura del filugello introdotta in Italia, prima nella Sicilia poi nell'Italia continentale. 
accoppiamenti erano come un segno del risvegliarsi della natura. Il costume inoltre di questi insetti di riunire le particelle escrementizie in masse sferiche e di farle rotolare senza posa come il Sisifo della mitologia, e qualche speciale forma del loro corpo, fecero si che i sacerdoti Egiziani li prendessero per simboleggiare le azioni di Osiride e del Sole. La figura dello scarabeo sacro si trova riprodotta in varie maniere in quasi tutti i monumenti Egizii, e l'immagine di questo Dio tutelare, come si puó vedere dai monumenti stessi, accompagnava gli Egizii in tutti gli atti della vita, e discendeva con essi nella tomba. Menzionerò fra gli insetti simbolici degli Egiziani ancora l'ape e la formica. La prima era simbolo di un re, del popolo governato e della sua prosperità e fecondità; la seconda era simbolo dell'intelligenza e della prudenza.

Noi possiamo, da quanto ho detto sopra, riassumere le cause che diedero origine alla entomologia in due sole: il tornaconto e la superstizione.

Originatosi cosi lo studio degli insetti, esso si venne col volgere dei secoli allargando e completando, e non sarà, credo, al tutto inutile che io scorra il più brevemente possibile attraverso alle vicende di questo studio dai tempi più remoti fino a noi.

La storia della entomologia si puó dividere in otto periodi più o meno caratterizzati e più o menó ben separati fra loro. Questi otto periodi, coi principali autori che li caratterizzano, sono i seguenti:

$1^{\circ}$ Periodo. Tempi anteriori ad Aristotile.

$2^{\circ}$ Aristotile, n. in Stagira 354 a. G. C., m. in Calcide, 2910 284 a. G. C.

Plinio, n. in Verona 23 a. G. C., m. nella eruzione del Vesuvio 79 dopo G. C.

$3^{\circ}$ Alberto Magno, n. 1193 in Colonia, m. 1281 in Colonia.

E. Wotton, n. 1492 in Oxford, m. 1555 in Londra.

G. Rondelet, n. 1507 in Montpellier, m. 1566 in Realmont presso Alby.

U. Aldrovandr, n. 1522 in Bologna, m. nel 1605 in Bologna.

T. Moufet, n. 1550 in Londra, m. 1604 in Bulbridge.

$4^{\circ}$ S. Swammerdam, n. 1637 in Amsterdam, m. 1685 in Amsterdam.

F. Redi, n. 1626 in Arezzo, m. 1697 in Pisa. 
M. Malpighi, n. 1628 in Crevalcuore, m. 1694 in Roma.

LeuWenhoeck, n. 1632 in Delft, m. 1723.

Sibilla di Merian, n. 1647 in Francoforte, m. 1717 in Amsterdam.

Réaumur, n. 1683 alla Rochelle, m. 1757 in Bermondière-surMeine.

DE GEER, n. 1720 in Finnspäng, m. 1778 in Stockholm.

LYoNNET, n. 1706 in Maestricht, m. 1789 in Jan.

BoNnet, n, 1720 in Genf, m. 1793 in Genf.

F. Huber, n. 1750 in Genf, m. 1831 in Prégny.

$5^{\circ}$ Linneo, n. 1707 in Roerhult, m. 1778 in Hanmarby presso Upsala.

Latreille, n. 1762 in Brives, m. 1833 in Parigi.

S. C. Fabricio, n. 1745, m. 1808.

Roesel, n. 1705 in Augustenburg presso Armstadt, m. 1759 in Nürimberg.

$6^{\circ}$ Cuvier, n. 1769 in Montbéliard, m. 1832 in Parigi.

E. Geoffroy St-Hilatre, n. 1779 in Estampes, m. 1844 in Parigi.

$7^{\circ}$ Savignx, n. 1777, m. 1851.

Désan, n. 1780 in Amiens, m. 1845 in Parigi.

Olıvier, n. 1756 in Fréjus, m. 1814 in Aalfort.

Lacordaire, $\mathrm{n}$. in Recey sul Ource 1801.

$8^{\circ}$ Darwin, n. 1807 in Schremburg.

Del primo periodo, vale a dire dei primissimi studii intorno agli insetti, ho già parlato.

Aristotile e Plinio caratterizzano il secondo. Aristotile fece per l'entomologia ció che aveva fatto per gli altri rami della zoologia; diede carattere di scienza alle cognizioni che al suo tempo si avevano intorno agli insetti, ed ebbe il grande merito non solo di aver data una classificazione degli animali, ma anche, cosa della massima importanza, di aver dato opera allo studio della loro interna struttura. Alcune delle divisioni degli insetti ed alcune delle denominazioni di Aristotile sono tuttora conservate nella scienza. Molti fatti inoltre intorno alla vita degli insetti che vennero scoperti in questi ultimi tempi, erano già conosciuti da Aristotile. La partenogenesi delle api, ad esempio, era in questo caso.

Gli insetti facevano parte, per Aristotile, degli animalia exsanguia minora. Egli aveva già riconosciuti i principali 
ordini degli insetti, e separati gli uni dagli altri; i coleotteri dalle elitre clure e resistenti, gli ortotteri dotati della proprietà di saltare, gli emitteri, i lepidotteri, gli insetti con due e con quattro ali membranose (che noi oggidi distinguiamo col nome di ditteri, di imenotteri e di neurotteri), gli insetti ora alati, ed ora atteri, come le formiche e le lampiridi, e finalmente gli atteri propriamente detti. Troviamo inoltre negli scritti di Aristotile cenni intorno alla struttura dell'apparato del canto della cicala (cosi dolce, come ci tramandò la storia, agli orecchi greci), e numerose ed interessanti osservazioni intorno ai costumi delle api.

Colla morte di Aristotile mori anche lo studio della storia naturale inteso come lo intendeva quel grande filosofo, fatto, vale a dire, mediante la conscienziosa osservazione del vero. L'impulso dato da Aristotile allo studio della zoologia cadde con lui, e vedremo quale lunga serie di anni passerá e quante lotte sarà necessario sostenere prima che lo studio della zoologia torni a mettersi sulla strada splendidamente segnata da Aristotile. Plinio che caratterizza, per ciò che riguarda lo studio della storia naturale, il periodo Romano, non segui l'esempio di Aristotile, e le sue opere si possono considerare come una semplice enciclopedia di cognizioni, tolte qua e lá, spesso senza criterio, intorno agli animali.

Caduto l'impero Romano segui un periodo di lotta fra l'antica civiltà pagana e il cristianesimo. In questo periodo di transizione che giunge fin verso il 1000 dell'êra volgare, non troviamo traccia di studii zoologici. La coltura del resto in quel tempo era poca e poco sparsa e limitata in massima parte al clero. Nei conventi in cui era allora ridotto tutto il sapere del tempo, si commentavano gli scritti di Aristotile e di Plinio, e si discuteva a perdita di vista intorno alle loro parole.

Passata in Occidente, per opera delle Crociate, gran parte della coltura araba, lo studio della storia naturale rinacque, e noi lo vediamo dar mano all'alchimia ed all'astrologia. Nel secolo dodicesimo troviamo già le opere di Alberto Magno, nelle quali sono riunite buona parte delle credenze e delle cognizioni di quel tempo intorno agli animali. Le opere di Alberto Magno, preziose per la storia della scienza, non portarono tuttavia a questa alcun serio giovamento.

Ben più importante è l'opera di E. Wotton, il quale, nel 
quindicesimo secolo, scrisse un libro intitolato De differentiis animalium (1), prezioso sotto varii rispetti.

Dopo il Wotton, spiccano fra gli altri, nel sedicesimo secolo, il Rondelet, il Moufet e l'Aldrovandi. Francesi i primi due, italiano il terzo.

Il Rondelet segui in parte l'esempio di Aristotile, e studio molti animali dal vero. Egli si occupò tuttavia principalmente degli animali marini e soprattutto dei pesci.

L'Aldrovandi diè opera anche allo studio degli insetti; ma i suoi scritti contengono molti errori, e la zoologia, direi, fantastica vi si trova sparsa a larga mano (2).

Molto importanti per la entomologia sono i lavori del Moufet (3), i quali ci dànno un'idea esatta dello stato dello studio degl'insetti in quel tempo.

Il Redi, lo Swammerdam, il Leuwenoeck, caratterizzano il quarto periodo.

Il quarto periodo dello sviluppo della entomologia è pure caratterizzato dalla caduta del principio autoritario. Il rispetto troppo religioso per l'autorità dei primi naturalisti, ed in ispecial modo di Aristotile, già alquanto scosso dal Rondelet, il quale, come ho detto, aveva voluto osservare egli stesso dal vero molti fatti intorno agli animali, venne nel secolo decimosettimo, per opera principalmente del Redi, completamente abbattuto. L'esempio del Redi, per quanto i suoi avversari abbiano fatto, non cadde, e venne raccolto dal Malpighi, il quale, primo fra tutti, portò la sua attenzione allo studio dell'interna struttura degli insetti, struttura che egli dimostrò, contro alla comune cre-

(1) Il Wotton aveva riconosciuto negli insetti un piano di struttura al tutto diverso da quello degli animali superiori e neque spinam habent ut pisces, dice egli parlando degli insetti nel libro sopracitato.

(2) \& De animalibus insectis, libri septem. In quibus omnia illa animalia accuratissime describuntur, eorum icones ad vivum ob oculos ponuntur, tandemque etiam natura mores ac proprietates ita declarantur ut quidquid de iis dici queat, facile inde innotescat. His adjunctus est index geminus capitum et verborum copiosissimus ». Bologna, 1602.

(3) «Insectorum sive minimorum animalium theatrum, olim ab Edoardo Wottono, Corado Gasnero, Thomaque Pennio inchoatum; tandem Tho. Moufeti Londinatis opera sumptibusque maximis concinnatum, auctum, perfectum, et ad vivum expressis iconibus supra quingentis illustratum ». Londini, 1634. 
denza di quel tempo, essere molto complicata. Egli in un celebre lavoro (1) pubblicatosi a Londra nel 1669, ci fa conoscere l'organizzazione del bombice del gelso in tutti i suoi stadii di sviluppo. Egli ci descrive i principali centri nervosi, le particolarità più notevoli del canal digerente, (certi vasellini speciali che accompagnano il canal digerente portano appunto il nome di vasi Malpighiani) gli organi della riproduzione; e, cosa molto importante, l'apparato respiratorio ed il cuore. Il Malpighi fece colle sue scoperte far un gran passo alla entomologia.

Si scopriva in questo tempo, per opera dell'olandese Leuwenhoeck, un istrumento il quale doveva avere per le scienze enormi conseguenze. Si scopriva cioè il microscopio e lo si applicava allo studio della struttura degli animali. L'opera del Leeuwenhoeck intitolata Arcana naturae detecta, ope microscopium (2), che portò nuova e splendida luce intorno alla struttura e soprattutto intorno al modo di propagazione degli animali, fu il primo e splendido risultato della importantissima scoperta. Un naturalista pure olandese, contemporaneo del Leuwenhoeck, Giovanni Swammerdam, porto pure, colla sua Storia generale degli insetti, largo contributo alla conoscenza delle metamorfosi e della struttura degli insetti. Poco mancò tuttavia che l'opera dello Swammerdam andasse perduta. Lo Swammerdam condusse una vita infelice e mori a quarant'anni, quasi ignorato prima di pubblicare la sua opera. Ún medico celebre, il Boerhaeve, raccolse i manoscritti dello Swammerdam e li pubblicò a proprie spese, col titolo stabilito dallo Swammerdam 'stesso di Biblia naturae (3). Questo lavoro è importantissimo e viene anche oggidi consultato con frutto.

Una donna intanto, Maria Sibilla di Merian, veniva al principio del $17^{\circ}$ secolo a portare non piccolo contributo allo

(1) "Dissertatio epistolica de Bombyce, societati regiae, Londini ad scientiam naturalem promovendam institutae, dicata ». Londini, 1669.

(2) « Delphis Batavorum». Kroneveld, 1695.

(3) "Bybel der natuure door Jan. Swammerdam Amsteldammer. Of. histoire der Insecten, etc. Joannis Swammerdamii Amstalaed. Biblia naturae; sive Historia Insectorum in classes certas redacta, nec non exemplis, et anatomico variorum animalculorum examine, aenisque tabulis illustrata Insectis numerosis rariorum naturae observationibus ». Leydae. 
studio degli insetti. Innamoratasi essa della storia naturale, con raro coraggio intraprendeva un lungo viaggio per l'America Meridionale, e per tre anni rimaneva nel Surinam, osservando e dipingendo gli insetti di quella località. Ritornata in Europa, mandava alle stampe un'opera splendida di disegni (1) in cui era raccolto il frutto delle sue osservazioni.

Le cognizioni intorno agli insetti, ed in generale intorno agli animali, si andavano cosi gradatamente aumentando. Queste cognizioni, tuttavia, venivano ad accrescersi moltissimo per l'opera di uno fra i più grandi osservatori che vanti la entomologia, per opera di Réaumur. Si fu in età di oltre cinquanta anni che il Rẻaumur cominciò la pubblicazione della sua grande opera intorno agli insetti, col modesto titolo di Mémoires pour servir à l'histoire des Insectes (2). È veramente straordinaria la quantità dei fatti osservati da questo grande naturalista, è veramente meravigliosa la diligenza e la sua abilità nello sperimentare, e la sua abilità e fedeltà nel riferire le osservazioni fatte. Non troviamo, è vero, negli scritti del Rẻaumur

(1) « Metamorphosis Insectorum Surimanensium. In qua Erucae ac Vermes Surimanenses cum omnibus suis transformationibus, ad vivum delineantur et describuntur, singulis eorum in plantas, flores et fructus collocatis, in quibus reperta sunt; tunc etiam Generatio ranarum, bufonum rariorum, lacertarum, serpentum, araneorum et formicarum, exhibetur; Omnia in America ad vivum naturali magnitudine picta atque descripta ». Amsterdami, 1705.

Sibilla di Merian scrisse pure un'opera íntorno agli insetti d'Europa. - Essa venne stampata in lingua Olandese nel 1730, e più tardi tradotta in Francese col titolo seguente: - "Histoire des Insectes d'Europe, dessinés d'après nature, et expliqués par Marie Sibille Merian; où l'on traite de la génération et des différentes métamorphoses des Chenilles, Vers, Papillons, Mouches et autres Insectes, et des Plantes, des Fleurs et des Fruits dont il se nourissent.

(2) Parigi, 7 vol. in- $4^{\circ}, 1734-1742$. Il Réaumur scrisse pure varie opere molto importanti intorno agli insetti. Sono da ricordarsi principalmente le seguenti:

" Histoire des Guêpes». Mém. Acad. des Sciences. Paris, 1719, T. 21 p. 230.

"Histoire des Teignes ou des insectes, qui rongent les laines et les pelletteries ». Mém. Acad. des Sciences. Paris, 1728, T. 30, p. 139.

«De la mécanique avec laquelle diverses espèces de Chenilles et d'autres insectes plient et roulent des feuilles des plantes e d'arbres, surtout celles du chêne ». Op. cit., 1730, vol. 32, p. 57.

* Observations sur les insectes qui se multiplient sans accouplement et par la seule fécondité de chaque individu ». Op. cit., 1741.

" Histoire du ver lion mouche ». 0p. cit., 1753, p. 402. 
la forma splendida e l'abilità oratoria del Buffon; ma vi troviamo, cosa molto più importante, l'esattezza nei particolari, vi troviamo l'impronta di un osservatore abilissimo e cocienzioso.

L'opera del Réaumur fece nascere in altri il desiderio di proseguire gli studii intorno agli insetti. Al Rẻaumur, tennero dietro, fra gli altri, il De Geer (1) ed il Lyonnet (2). Il primo continuó le osservazioni del Réaumur, il secondo diede alla scienza un'opera intorno all' anatomia del bruco che rode il legno dei salici, opera che il Cuvier chiamò il capolavoro della anatomia e della incisione. $\dot{E}$ veramente meravigliosa la diligenza e l'abilità spiegata dal Lyonnet in questo studio. Egli ci rappresenta in tutti i loro particolari, i nervi, i muscoli, e i visceri della larva citata, con disegni molto esatti ed eleganti. Al tempo del Lyonnet l'utilità dei disegni, dirò di passaggio, nei lavori di storia naturale era già riconosciuta, i disegni tuttavia, erano grossolani, poco precisi e non corrispondevano allo scopo; erano anzi d'impaccio più che non di aiuto alla descrizione. Il Lyonnet volle incidere di propria mano le tavole del suo lavoro, le quali riuscirono realmente, come disse il Cuvier, meravigliose.

Nel diciottesimo secolo, per opera principalmente degl'insigni naturalisti or ora citati, lo studio della storia naturale si era alquanto diffuso, e gli osservatori si erano moltiplicati. Ricorderò di sfuggita il ginevrino Carlo Bonnet (3), il quale fece ricerche intorno alla respirazione delle larve del filugello ed intorno al modo di generazione degli afidi. Ricorderò anche Francesco Huber (4),

(1) «Mémoires pour servir à l'histoire des Insectes ». Stockholm, 7 vol., 17521778. Il De Geer, oltre a quest'opera capitale scrisse pure molte piccole memorie che vennero stempate nelle Memorie dell' Accademia di Parigi.

(2) "Traité anatomique de la Chenille qui ronge le bois de saule ". - La Haye, 1760.

(3) "Recherches sur la respiration des Chenilles. Mémoire Math. des Savants étrangers ». Paris, 1768, p. 276, vol. 5.

"Observations sur les stigmates des Papillons». 1768, vol. 5, p. 294.

- Lettre et Mémoire sur les Abeilles ». Journ. de Phys., 1775.

"Aphides variae». Eurres de Bonnet, tom. I.

(4) « Nouvelles observations sur les Abeilles ». Genève, 1792. - Notiamo pure fra gli scritti dell'Huber i seguenti:

"Sull'origine della cera ». Nuova scelta d'Opere, inter. 1804, tom. I, p. 52. 
pure ginevrino, il quale, cieco già dalla giovinezza, seppe tuttavia, servendosi di un servo devoto ed intelligente, condurre a termine la più bella e la più completa storia dei costumi delle api che si abbia anche oggidi.

Eccoci giunti così ai periodi più splendidi dell'entomologia, periodi che sono caratterizzati dai nomi illustri di Linneo, di Fabricio e di Cuvier.

Io non starò qui ad enumerare i meriti di Linneo, essi sono noti a tutti, e tutti sanno che egli fu il legislatore della zoologia e della botanica, tutti sanno di quale importanza per queste scienze sia stata l'applicazione ad esse della nomenclatura binomia.

Linneo si occupó molto degl'insetti ; ma le sue classificazioni sono classificazioni artificiali.

Al cittadino Latreille (1796) (1) spettava l'onore di tentare una prima distribuzione degl'insetti secondo l'ordine naturale, tenendo conto non più di un carattere qualunque, ma della somma delle rassomiglianze, del complesso dei caratteri.

In quanto a Fabricio, discepolo di Linneo, egli segui gli studii del maestro intorno agli insetti, e ne diede una classificazione fondata sopra gli organi masticatori (2). Egli commise molti errori, ma l'opera sua giovò tuttavia assai al progresso dell'entomologia.

"Sulle formiche, uso delle loro antenne, e loro rapporti coi pidocchi delle piante e coi gallinsetti ». Op. cit., p. 206.

" Sulla generazione delle Api ». Op. cit., p. 212.

(1) « Précis des Caractères génériques des insectes, disposés dans un ordre naturel, par le citoyen Latreille». Brive, Bordeaux, an. V (1796).

I lavori del Latreille sono molti, ed alcuni voluminosissimi. Ricorderò, fra gli altri, i seguenti:

"Histoire naturelle générale, et particulière des Crustacés et des Insectes » 14 volumi, dall'anno X all'anno XIII (1802-1805).

* Genera Crustaceorum et insectorum secundum ordinem naturalem in familias disposita, iconibus exemplisque plurimis explicata ». Parisiis et Argentorat, 4 vol., 1806-1809.

(2) «Systema Entomologicae sistens insectorum larves, ordines, genera, species, adjectis synonymis, locis, descriptionibus ». Flensburgi et Lipsiae, 1775.

"Entomologia systematica emendata et aucta, secundum classes, ordines, genera, species, etc. ». Hafniae, vol. 4, 1792-1794. 
Non voglio lasciare di citare il nome di un naturalista che visse in quel tempo, vale a dire A. J. Roesel. Il Roesel, diligentissimo osservatore e nello stesso tempo abilissimo disegnatore e colorista, ci lasciò parecchi volumi illustrati da stupendi disegni in cui sono registrate le osservazioni da lui fatte.

Il Roesel, a quanto pare, non dava molta importanza ai suoi studii intorno agli insetti, ed intitolava l'opera sua intorno agli insetti Insecten Belustigung (Divertimento intorno agli insetti) (1). Mentre, si noti, un'altra sua opera, non meno ammirabile per disegni e per abilità di osservazioni intorno agli anfibii anuri, chiamava Historia ranarum. Ciò, del resto, era in armonia colle idee di quel tempo, in cui i vertebrati soli si stimavano degni della osservazione e dello studio del filosofo.

Fino a quel tempo gli entomologi si erano per lo più limitati allo studio delle parti esterne degli insetti, e questo studio era stato portato molto avanti dal Latreille. Poco conosciuta invece era la struttura interna degli insetti. I lavori del Malpighi, del Bonnet, del Lyonnet e di altri, erano isolati e non potevano dare alcuna idea generale intorno alla struttura, intorno alle funzioni, in una parola, intorno alla vera essenza degli insetti.

Le classificazioni inoltre fino verso il finire del passato secolo avevano per oggetto la semplice determinazione delle specie. Il Cuvier fu il primo a stabilire l'importantissimo principio che la classificazione doveva dare una fedele e chiara idea delle affinità degli animali fra loro. Egli gettò le basi dell'anatomia comparata, e per mezzo di questa potè vedere come tutti gli animali si possano raggruppare intorno a quattro tipi principali.

Alle teorie di Cuvier, venivano poco dopo a contrapporsi quelle di un altro grande naturalista, il Geoffroy di St-Hilaire, il quale invece dei quattro tipi sosteneva l'idea dell'unità della costituzione degli animali.

Questa idea che diè luogo a tante discussioni fra il Cuvier ed il Geoffroy di St-Hilaire venne con grande giovamento

(1) «Der monatlich heraugegebenen Insecten Belustigung » 4 Parti, 17461761. Nürinberg. 
delle scienze accolta dal Savigny, il quale, compagno al Geoffroy nella spedizione d'Egitto del Bonaparte, si rese celebre per la pubblicazione di un lavoro intorno ai materiali entomologici raccolti in quel viaggio. A questa gloria, tuttavia, egli ne aggiunse in breve un'altra ben più grande. La sua opera intitolata Theorie des organes de la bouche des animaux invertébrés et articulés compris par Linné sous le nom d'insectes, pubblicatasi nel 1816 intorno alla teoria degli organi della bocca degli animali articolati, caratterizza veramente un periodo della storia della entomologia. Egli dimostrò come negli insetti e nei crostacei vi sia un piano generale di conformazione, e come in essi, ad esempio le mandibole e le mascelle, la proboscide succiante, ecc., non siano altro che trasformazioni di un dato numero di parti che si trovano in tutti gli insetti.

La teoria del Savigny o delle omologie esiste tuttora ed ha portata molta luce sopra un argomento molto difficile ed intricato. La scienza non potè sventuratamente trarre da questo grande naturalista tutto l'utile che si sarebbe potuto sperare. Divenuto cieco, condusse per 27 anni una vita piena di crudeli dolori fisici e morali.

Le idee intorno agli animali si erano intanto andate allargando e completando per opera dei naturalisti sopra citati. Si cominciavano a travedere leggi regolatrici delle forme animali, e del prodursi delle modificazioni degli organi; si cominciava in una parola a guardare sotto un gran punto di vista sintetico l'immensa congerie dei fatti osservati intorno agli animali.

Verso il principio del corrente secolo una scienza nuova sorgeva, l'embriologia, la quale studiando lo sviluppo degli animali portava intorno alla conoscenza degli organi ed intorno al loro variare idee più giuste ed esatte. Le metamorfosi degli insetti vennero diligentemente studiate e troppo lungo sarebbe l'enumerare tutti gli scrittori che si sono occupati di questo argomento. Si fu pure in principio del corrente secolo che il Baer ed il Rathke fecero i loro studii di embriologia. Il Baer giunse a conclusioni analoghe a quelle di Cuvięr. Egli riconobbe cioè nel regno animale quattro maniere principali di sviluppo, le quali corrispondono ai quattro tipi del Cuvier. Un anno dopo la pubblicazione del Baer, il Milne Edwards, fa- 
cendo ricerche intorno ai crostacei, annunziava un fatto di importanza capitale; che cioè nei primi stadi dello sviluppo tutte le specie dello stesso genere si rassomigliano e che le distinzioni fra specie e specie non si manifestano che nel progressivo sviluppo dell'animale.

Cadeva per queste ricerche una teoria la quale per molto tempo aveva tenuto il campo della scienza, la teoria, voglio dire, della catena degli esseri di Leibnitz e di Bonnet.

Verso il principio del corrente secolo gli entomologi cresciuti in numero cominciarono a dividersi in due schiere. Gli uni seguendo la scuola del Redi, del Malpighi, dello Swammerdam e del Roesel, si occuparono dei costumi e della struttura degli insetti. Gli altri rivolsero la loro attenzione alla caratterizzazione e alla descrizione esatta delle specie senza curarsi d'altro.

L'Europa allora più non bastó, e l'America, l'Africa, l'Asia portarono alla entomologia il loro splendido tributo. Naturalisti e pittori con febbrile attività si diedero a descrivere ed a rappresentare col disegno le nuove forme. Il numero delle specie di insetti descritti aumentó cosi rapidamente. Citerò a questo proposito un esempio.

Nel primo catalogo di coleotteri pubblicatosi dal conte di Dejan nel 1802 erano annoverate 910 specie. Nella $2^{a}$ edizione dello stesso catalogo stampatosi nel, 1821, il numero delle specie era già salito a 6692 , nella $3^{\mathrm{a}}$ stampatasi solo cinque anni dopo, le specie erano 20,909. Nell'ultima poi pubblicatasi un solo anno dopo le specie erano in numero di $22,399$.

Dal 1837 ai nostri giorni questo numero si è quasi triplicato e non si va lungi dal vero, facendo ascendere ad una sessantina di migliaia le specie di coleotteri conosciute. Si faccia ora il calcolo dei lepidotteri, degli ortotteri, ecc., e il numero delle specie degli insetti conosciuti sale a parechie centinaia di migliaia.

La moda s'impadroni essa pure degli insetti ed i più belli fra questi vennero trasformati in oggetti di ornamento. Si videro allora le scintillanti crisomele e gli splendidi buprestidi (1) legati in oro ed in argento servire da bottoni da

(1) Fra i coleotteri sono più in uso anche al giorno d'oggi i seguenti: Hoplia farinosa, comunissima nella Francia centrale - Chrysochroa bivettata e dives 
camicie, e i fosforescenti elateridi della America meridionale ornare di tremule luci le vesti delle signore, mentre le stupende farfalle azzurre appartenenti al genere Morpho, colle loro ali dai riflessi madreperlacei ne ornavano le chiome (1).

Molto tempo prima della moda, del resto, la cucina si era impadronita degli insetti. Sarebbe certamente cosa molto interessante il passare in rivista tutti gli insetti che presso i varî popoli nei tempi si antichi che recenti servirono e servono tuttora come cibo. Io mi limito tuttavia a riferire un elenco dei principali.

\section{Eleneo delle principali specie di insetti}

che presso $i$ varii popoli servirono e servono tuttora di cibo.

\begin{tabular}{c|c|c|c}
\hline Generi & Specie & Localitá & $\begin{array}{c}\text { Antori } \\
\text { che hanno osservato } \\
\text { il fatto }\end{array}$ \\
\hline
\end{tabular}

\section{Coleotteri.}

\begin{tabular}{c|c|} 
Scarabeus & sacer Linn. \\
Oryctes & boas Fab. \\
Lucanus & cervus \\
Prionus & coriarius \\
Macrodontia & cervicornis \\
Calandra & palmarum
\end{tabular}

Egitto
Sierra Leone
Europa
Europa
America
Indie Orientali

Dott. Clarke Lane. Luogotenente Sayer. Scopoli. Amoreux. Linneo. Linneo e Sibilla di Merian.

\section{Ortotteri.}

\begin{tabular}{|c|c|}
\hline Locusta & migratoria \\
\hline$»$ & \\
\hline » & gregaria \\
\hline » & cernensis \\
\hline$\gg$ & mahrattarum \\
\hline 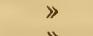 & viridissima \\
\hline$\gg$ & persarum \\
\hline
\end{tabular}

\begin{tabular}{|c|} 
Crimea \\
Egitto, Arabia \\
Arabia \\
Madagascar \\
Indostan \\
Europa \\
Persia, \\
(Acridophagi)
\end{tabular}

Linneo.

Erodoto. Aristotile. Ives's Travels. Forbes, Dampier. Id. Morier.

della China - Pachyrhyncus perlatus delle isole Filippine - l'Eutimus imperiatis e l'E. augustus del Brasile - Colaspis flavipes, ecc., Cassida variolosa, Praepodes Regatis, ecc.

(1) Erano in Francia, non è gran tempo, molto in uso il Morpho Rhetenor e Cypris che entravano a far parte delle acconciature delle signore, e costavano oltre a 250 franchi l'uwo. Altre specie non meno belle, ma più a buon mercato, erano pure molto in uso, fra queste principalmente i $M$. Menelas, Adonis, ecc. 


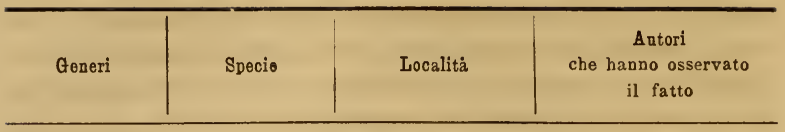

Rincoti.

\begin{tabular}{c|c|c} 
Tettigonia & antiquorum & Grecia \\
Eliano.
\end{tabular}

\section{Lepidotteri.}

\begin{tabular}{c|c|c|c} 
Papilio & (larve) & Ottentotti, China & $\begin{array}{c}\text { Sparman, } \\
\text { G. Stauton. } \\
\text { Cossus }\end{array}$ \\
liguiperda & Romani & Ray, Linneo.
\end{tabular}

Neurotteri.

\begin{tabular}{c|c|c|c}
$\underset{\text { Termes }}{»}$ & $\begin{array}{c}\text { fatale } \\
\text { arborum }\end{array}$ & $\begin{array}{c}\text { Caffreria } \\
\text { America Merid. }\end{array}$ & $\begin{array}{c}\text { Lichtenstein. } \\
\text { Koster. }\end{array}$
\end{tabular}

\section{Imenotteri.}

\begin{tabular}{c|c|c|c} 
Apis & mellifica & Barbaria Occid. & Dott. Halley. \\
Formica & cupia & America Merid. & Piso. \\
$»$ & $?$ & Ceylan & Humbold. \\
$»$ & $?$ & Callaway.
\end{tabular}

\section{Ditteri.}

Musca

$\gg$ $\stackrel{?}{\text { larve }}$
America Merid.

Europa
Pinto. Scopoli.

Dal soprascritto elenco si scorge come tutti gli ordini degli insetti abbiano dato e diano specie che servono di cibo all'uomo. Chi desidera maggiori particolari intorno a questo argomento consulti fra gli altri autori il Rev F. W. Hope (Observations respecting various Insects which at different times have afforded Food to Man Trans. Ent. Soc. London 1841-43, vol. III, pag. 129), dal quale scritto io ho tolto in parte l'elenco soprascritto. Si veda anche a questo proposito Piero Bargagli (Insetti commestibili, Rivista Europea. Firenze 1877, vol. II, fasc. V).

Tornando alla entomologia descrittiva diró come al principio del corrente secolo avessero il primato in questo genere di studi la Francia e l'Inghilterra. I lavori di Latreille, Olivier, Bosc, di Déjan, di Lacordaire, di Godart, di Duponchel, di Lucas, di Curtis, di Hope, di Westwood, di Doubbday e di 
molti altri, che qui sarebbe troppo lungo nominare, ne fanno fede. Sarebbe certamente cosa molto interessante ed istruttiva l'esaminare la parte che ciascuno dei grandi entomologi che ora ho citato ha avuto nel progresso della scienza: io mi limiterò tuttavia a dire un po' più minutamente di uno dei più celebri collettori e descrittori di questo periodo, del conte Déjan.

Il conte Déjan nacque in Amiens nel 1780. Cominciò a quindici anni la vita del soldato e prese parte alla maggior parte della grandi battaglie della rivoluzione e dell'Impero. Aiutante di campo di Napoleone I venne proscritto alla caduta dell'Impero e non rientró in Francia che nel 1818. La rivoluzione di luglio gli fece riprendere la vita degli affari che continuó fino al 1845, anno in cui mori.

L'amore per lo studio degli insetti sorse di buona ora in Déjan, il quale ebbe la fortuna di avere a guida dei suoi primi studi il Duméril. Questo amore per la entomologia non scemò mai in lui e lo segui in tutte le circostanze della sua vita fortunosa. Mi piace anzi di raccontare un fatto, il quale dà una idea chiara dell'indole di questo grande entomologo. Dall'anno 1802 al $1815 \mathrm{fu}$, come tutti sanno, un succedersi rapidissimo di guerre in Spagna, in Portogallo, in Austria, in Russia, e Francia. Il Déjan, durante queste guerre a cui egli prese parte attiva, non dimenticò gli insetti, e negli ozii del campo, ozii molto brevi a dir vero in quel tempo, durante le marcie, e sullo stesso campo di battaglia, egli non cessò mai di raccogliere insetti. Racconta egli stesso che aveva foderato con uno straterello di sughero la parte interna del suo elmo nel quale infilzava gli insetti che gli veniva fatto di trovare. Una volta fra le altre alla battaglia di Alcanizar egli scorge su di un fiore presso un piccolo ruscello, al momento di dare il segnale di caricare il nemico, un Cebrio ustulatus. Egli discende senz'altro da cavallo, infilza l'insetto nel suo elmo e carica il nemico. Nella battaglia l'elmo di Déjan venne maltrattato dalla mitraglia, ma con grande gioia di Déjan il Cebrio era rimasto intatto.

Dei lavori entomologici di Déjan io accenno a due soli, il catalogo dei coleotteri della sua collezione, di cui si pubblicarono varie edizioni, opera a cui principalmente si deve l'impulso grandissimo che prese allora lo studio dei coleotteri e 
lo Species, opera gigantesca, che secondo le idee dell'autore doveva comprendere la descrizione di tutti i coleotteri conosciuti. Egli non ne pubblicò che cinque volumi; questi tuttavia bastano alla gloria del Déjan. La sua collezione, la più vasta e completa che allora esistesse, egli stesso la vendè verso gli ultimi anni della sua vita, in cui i malanni e soprattutto la vista indebolita gli impedivano di poter proseguire lo studio degli insetti. La sua collezione venne venduta divisa in vari lotti. Una parte di essa si conserva preziosamente nel museo zoologico di Torino.

Mentre al principio del corrente secolo da una parte crescevano i collettori ed i descrittori, diminuivano invece gli entomologi, che si occupavano dei costumi e della struttura degli insetti, e ciò con danno della scienza, imperocchè la conoscenza delle parti esterne di un animale non basta per darci un concetto giusto dell'animale stesso. Si hanno tuttavia anche in questo tempo osservatori abilissimi che fecero osservazioni della massima importanza a questo proposito.

Ricorderò i lavori di Audonin, di Milne Edwards e di Carus intorno al sistema nervoso ed alla circolazione del sangue dei crostacei e degli insetti, il maraviglioso lavoro dello Straus Durkéim intorno alla anatomia della comune melolonta e i numerosi lavori di Leon Dufour e di Giorgio Newport intorno alla anatomia di un grande numero di insetti.

Il numero grande di naturalisti che in principio del corrente secolo e sul finire del precedente si occupavano dello studio degli insetti fece nascere in breve il bisogno di riunirsi per discutere certe questioni, per intendersi sopra certi punti di scienza. Questo bisogno fu la causa della formazione delle società entomologiche che caratterizzano gli ultimi due periodi della storia della entomologia. Il primo tentativo di società avente per iscopo lo studio degli insetti di cui si abbiano notizie sicure, è la Società Aureliana di Londra di cui si ignora l'anno preciso della fondazione, ma si sa tuttavia con certezza che fioriva verso l'anno 1745. I soci si riunivano per le discussioni nell'osteria del Cigno.

Un incendio che distrusse il luogo di riunione, mandò a male la società. - Nel 1762 se ne fece un'altra collo stesso nome, la quale tuttavia non durò molto. 
La Società Entomologica di Londra venne fondata nel 1780 , e durò due anni. Nel 1801 si formó la terza Società Aureliana che cadde anch'essa nel 1806. Nel 1806 si fece la Societá Entomologica, la quale pubblicò nel 1812 tre fascicoli col titolo di Transactions. In Francia, si fu nel 1832 che ebbe origine la Société Entomologique de France, la quale, insieme colla Società Entomologica di Londra, costituita nel 1833, tenne per molto tempo il primato negli studii entomologici per le sue importanti pubblicazioni.

Molte altre società entomologiche si formarono posteriormente a queste citate in quasi tutte le nazioni più civili di Europa e di America. Alcune divennero celebri e sono tuttora fiorenti. Numerose e splendide pubblicazioni intorno agli insetti furono il frutto delle società entomologiche, pubblicazioni che certamente contribuirono moltissimo all'avanzamento della scienza.

L'ultimo periodo in cui ho detto potersi dividere la storia della entomologia, è quello che è cominciato poco dopo la metå del corrente secolo e che si continua tuttora. Questo periodo si puó chiamare Darviniano.

Le nuove idee del Darwin esposte intorno alla specie, e le nuove leggi da lui scoperte, hanno aperti nuovi ed immensi campi alla osservazione ed hanno chiamato l'attenzione degli osservatori sopra certi fatti che per lo addietro erano trascurati o male interpretati. Le leggi della scelta naturale, della scelta sessuale, del mimismo, ecc., hanno modificato il concetto della specie, ed hánno dato una spiegazione soddisfacente di un grande numero di fatti.

La teoria di Darwin e della discendenza degli esseri ci fa conoscere le cause efficienti delle forme organizzate, e mentre prima di Darwin la zoologia e la botanica non si occupavano delle forme organiche, che come di cose isolate, senza darsi pensiero delle cause che hanno prodotto e che producono queste forme, le teorie darviniane ne vennero a spiegare soddisfacentemente la formazione.

Darwin non è il primo che abbia tentato di dare una interpretazione meccanica della origine delle forme animali e vegetali. È il primo tuttavia che sia riuscito a darla in modo chiaro ed a corredarla di dimostrazioni fondate sull'esame dei 
fatti. La teoria della evoluzione infine ci concede di farci un concetto chiaro della unità della natura in modo che noi possiamo spiegare per mezzo di cause meccaniche i fenomeni complicati del mondo organico nello stesso modo in cui si spiegano gli altri fenomeni fisici.

Gli insetti hanno fornito al Darwin, al Wallace e ad altri che si sono occupati della teoria della evoluzione, un grande numero di fatti ed un campo vastissimo di osservazione. Ed è certamente negli insetti, pel rapido succedersi delle generazioni, che le mutazioni che avvengono nelle specie o per legge di eredità o di adattabilità si possono manifestare più rapidamente e più profondamente.

L'Italia ebbe nello sviluppo della entomologia una parte molto importante, e basta che io ricordi i nomi di Redi, di Malpighi, di Spallanzani, perchè la mente ricorra con compiacenza a quei tempi splendidi per la scienza italiana in cui vivevano quei grandi. Gli autori citati appartengono alla categoria di quelli che si occuparono dello studio dei costumi degli insetti. L'Italia tuttavia non è priva di entomologi appartenenti alla seconda. In questa spicca soprattutto il Piemonte, nel quale, più che non in altra parte d'Italia, da oltre un secolo lo studio della entomologia conta insigni cultori.

In questo secolo e sul finire del precedente noi troviamo a Napoli il Costa, in Toscana il Rossi, a Milano il Bassi, a Genova lo Spinola. In Piemonte poi troviamo il Giorna, il Bonelli, il Gené, il Truqui, ed il Ghiliani. Il più celebre di tutti è certamente il Bonelli, il quale per primo ebbe il grande merito di aver dato una classificazione razionale dei carabici che in gran parte è ańcora in uso al giorno d'oggi. Un altro merito sommo del Bonelli si fu quello di aver compreso l'importanza delle faune locali e di aver cominciato a riunire materiali per una fauna entomologica del Piemonte. Il Genẻ ed il Ghiliani vanno celebri per i loro studi sulla fauna entomologica della Sardegna. Il Ghiliani poi, di cui piangiamo la recente perdita, riprese l'opera del Bonelli, e per tutta la sua vita con invitta pazienza e con invitto ardore raccolse e studio gli insetti del Piemonte. Le sue raccolte si conservano gelosamente nel Museo di Torino in pro del quale egli spese tutta la sua laboriosa vita. Egli non pubblicó che il resultato delle 
sue osservazioni sui lepidotteri. Questa pubblicazione è più che sufficiente tuttavia a collocare il Ghiliani fra i più insigni entomologi italiani.

\section{Bibliografia.}

G. Cuvier. - Histoire des sciences naturelles depuis leurs origine jusqu'à nos jours, chez tous les peuples connus. 1845.

Gravenhorst. - Dissertatio sistens conspectum Historiae Entomologicae, ecc., 1801.

LAPORTE. - Essai pour servir à l'Histoire des sociétés entomologiques. Ann. Soc. Ent. Fr. 1837.

Latreille. - De l'origine et des progrès de l'Entomologie. Mem. d. Museum, vol. VIII, 1822.

- Des insectes peints ou sculptés sur les monuments de l'Egypte. Op. cit. 1919. Fabricius. - Phisophia entomologica.

Groshans. - Prodromus Faunae Homeri et Hesiodi. Lugduni Batavorum. 1839. KLopscr. - Enstehung der Insecten nach Aristotiles. Isis., 1834.

HoPE. - On the Notions entertained respecting the Emblem. Scarabaeus. Trans. Ent. Soc. Lond. 1839.

II De Locke, il Mac Lay, il Mulsant, il Keferstein, il Clark, ecc., hanno pure qualche scritto più o meno importante, che riguarda le cognizioni degli antichi intorno agli insetti. 


\title{
II.
}

\section{GLI INSETTI}

\author{
IN RAPPORTO COLL'AGRICOLTLRA.
}

Prima di entrare a discorrere minutamente degli insetti io devo rispondere ad una domanda che probabilmente viene spontanea alle labbra di parecchi dei miei lettori.

A che cosa serve, mi si puó domandare, lo studio degli insetti? Quale è l'applicazione pratica della entomologia? Io potrei, pigliando ora l'occasione da questa domanda, fare una lunga dissertazione intorno alla importanza della scienza pura, e potrei dire, come lo studio e la ricerca del vero non abbia bisogno per essere fatto di uno scopo pratico, e come esso basti a sè stesso. Io tuttavia non insisterò sopra questo argomento, imperocchè io credo che tutti o quasi tutti i miei lettori siano persuasi della importanza della scienza pura. A quelli tuttavia fra i miei lettori che per aventura non lo fossero, io consiglio:

Di leggere uno scritto del Vallisnieri intitolato: Della nobiltà ed utilitá dello studio degli insetti (Op. fis. med., vol. I, p. 307);

Di meditare le parole che un illustre fisico inglese, John Tyndall, pronunziava in una lezione fatta al popolo più pratico del mondo, agli Americani, e che si trovano stampate nel suo libro intitolato La luce, e soprattutto poi vorrei che ricordassero le parole seguenti che il Cuvier ha detto intorno al nostro argomento:

"Ces grandes innovations pratiques sont la pure application des vérités d'ordre élévé qui n'ont pas été cherchées dans une intention pratique, mais qui ont été poursuivies pour ellesmêmes et par le seul amour de la science. Ceux qui les appliquent n'auraient aucune inclination à les conduire à une 
fin pratique. Engagés dans les hautes régions où leurs pensées les ont emportés, il poursuivraient difficilement les résultats pratiques, quoique nés de leurs propres nécessités. Ces ateliers qui s'élèvent de toutes parts, ces colonies si peuplées, ces vaisseaux qui sillonnent les mers, cette abondance, ce luxe, ce tumulte, tout cela vient des inventeurs de la science, et cependant tout cela leur paraît étrange ».

Per ciò che è della entomologia, sono note a tutti le principali sue applicazioni alle arti ed alle industrie. Tutti conoscono il filugello, le cocciniglie, le api, le cantaridi, ecc. Io non insisterò intorno a ciò, mi fermerò in vece un po' più a lungo a trattare dei rapporti che l'entomologia ha colla agricoltura, questione molto complessa e difficile ed intorno alla quale si fa oggi un grande discutere, ed intorno alla quale la comune della gente ha idee inesatte e confuse.

L'entomologia ha colla agricoltura legami strettissimi, e lo studio degli insetti in quanto essi sono utili o nocevoli alle varie sorta di piante dall'uomo coltivate è della massima importanza ed assai difficile. L'argomento è vastissimo, io non fo che accennarlo, imperocchè esso ci trarrebbe troppo fuori del nostro campo.

L'uomo, come è ben naturale, cercò in tutti i tempi di impedire i danni che gli arrecano gli insetti. La quantitá di opere che si sono scritte intorno agli insetti dannosi od utili alla agricoltura è veramente enorme. Tutte le nazioni più civili di Europa e di America, soprattutto in questi ultimi tempi, si sono dato pensiero di questa importantissima questione ed hanno promosso la formazione di società aventi per scopo speciale lo studio degli insetti considerati sotto questo punto di vista. Ma fino ad ora, non ostante gli sforzi dei molti naturalisti che se ne sono occupati, si è conchiuso ben poco e gli insetti continuano a distruggere i nostri vigneti, i nostri cereali senza dársi il benchè minimo pensiero delle nostre dissertazioni.

Io aggiungo subito, tuttavia, che la distruzione degli insetti nocivi all'agricoltura è impresa difficile e penosa come ogni altra che si propone di andar contro alle leggi di conservazione e di riproduzione delle specie. - Mentre l'uomo distrugge cento, la natura riproduce mille. 
Una ragione inoltre dello scarso risultato finora ottenuto intorno alla questione degli insetti nocivi all'agricoltura dobbiamo ricercarla nel relativamente piccolo numero di fatti e di esperimenti ben sicuri, nelle scarse cognizioni che si hanno dei costumi di molte specie di animali e nella persistenza di certe teorie erronee, ma che tuttavia vengono ancora da alcuni sostenute.

In questa, più forse che non in nessuna altra parte delle scienze naturali, è necessaria l'opera di molti e principalmente degli agricoltori, di quelli cioè che continuamente, per dir cosi, sul luogo dell'azione possono seguire i fatti in tutte le loro fasi. Al naturalista poi tocca di riunire tutti i dati dell'osservazione e quindi dedurre leggi generali, le quali tanto più si accosteranno al vero quanto maggiore sarà il numero dei fatti sopra cui si fonderanno. $\dot{\mathrm{E}}$ necessario soprattutto che le osservazioni e gli esperimenti vengano ripetuti il più possibile e nel maggior numero possibile di località diverse, tenendo conto di tutte le cause che possono esercitare una qualche azione sui varî fenomeni. In questo ordine di fatti l'analogia ha poca importanza e poco valore.

Le difficoltà che si incontrano nella distruzione degli insetti dannosi si possono dividere in due grandi categorie, in difficoltà intrinseche ed in difficoltà estrinseche. Le intrinseche, dirò col Costa di Napoli, che scrisse un bellissimo libro intorno agli insetti nocevoli agli olivi ( 1 ); " sono quelle che risultano dal costume stesso dell'animale, dalla parte vegetale che attacca, e da quanto altro puó riguardarlo. Tali difficoltà esser debbono vinte dalla scienza. A tale uopo si rende indispensabile la esatta e compiuta conoscenza della biologia di ciascuna specie » (2). Fino a tanto che noi non conosceremo bene il modo di vivere di un dato insetto, sarà vano proporre mezzi per distruggerlo. La scienza pura deve precedere l'applicazione. Conosciuti i costumi di una data specie di insetto nociva, la scienza consiglia varî mezzi per impedire i danni e per distrug-

(1) A. Costa, "Degli insetti che attaccano l'albero ed il frutto dell'ulivo, del ciliegio, etc. ». Napoli, 1857.

(2) Op. Cit., p. 12. 
gere la specie stessa. L'attuazione dei mezzi proposti dalla scienza, il che è còmpito non più del naturalista, ma dell'agricoltore pratico, presenta moltissime difficoltà, le quali costituiscono appunto le difficoltà estrinseche sopracitate.

I mezzi per cui gli insetti dannosi possono venire, se non intieramente distrutti, ridotti almeno tanto in numero da non portare danno sensibile alle piante coltivate dall'uomo, si possono dividere in mezzi che diremo naturali, nei quali l'uomo non ha azione alcuna, ed in mezzi che l'uomo stesso puó mettere in pratica.

Io non mi fermerò intorno ai secondi, dirò invece un po' più lungamente dei primi.

Si è detto e si è sostenuto accanitamente per lungo tempo che gli uccelli insettivori, i mammiferi insettivori ed i rettili sono i più efficaci ausiliari dell'uomo nella guerra contro agli insetti.

Questa opinione venne sostenuta da parecchi entomologi illustri, i quali ragionavano cosi: Vediamo crescere il numero degli insetti che annualmente devastano le nostre campagne, e vediamo scemare quello degli uccelli per la distruzione che se ne fa: ora siccome gli uccelli in gran parte si pascono d'insetti, lasciateli crescere in numero e gli insetti decimatori dei nostri prodotti agricoli scompariranno. Queste teorie fecero si che in quasi tutte le nazioni si prendessero dai governi provvedimenti speciali per proteggere gli uccelli. Questi provvedimenti non sono certamente da rigettare, imperocchè valgono ad impedire la smodata distruzione degli uccelli, ma non raggiungono punto il loro scopo e anche nei paesi dove vengono osservati col maggior rigore, gli insetti continuano l'opera loro come se niente fosse. Per ben comprendere come le teorie sopra enunziate siano erronee, conviene che noi facciamo anzitutto alcune considerazioni generali.

Considerando tutti gli insetti ed in generale tutti gli animali noi vediamo:

$1^{\circ}$ Che si possono dividere in due grandi schiere: animali carnivori ed animali fitofagi;

$2^{\circ}$ Che le specie animali si sviluppano in ragione diretta del nutrimento che è a loro disposizione.

Ne segue da ciò che sia le specie carnivore, sia le specie 
fitofaghe non possono svilupparsi al di là di un certo limite, oltrepassato il quale, esse distruggono la sorgente stessa del loro nutrimento, e l'equilibrio, rotto dall'eccessivo sviluppo dell'una e dell'altra sorta di animali viene cosi ristabilito.

Affinchè la legge ora enunciata appaia ben evidente ricorriamo ad un esempio pratico. Consideriamo una porzione qualsiasi di terreno incolto, supponiamo che in esso crescano molte sorta di erbe e di piante selvatiche, visitando questo terreno noi lo troveremo popolato da un grande numero di insetti, gli uni fitofagi che si nutrono a spese delle varie sorta di piante che colà crescono, gli altri carnivori che vivono alle spese delle specie fitofaghe. Supponiamo che per una qualche cagione le specie carnivore aumentino straordinariamente in numero, esse in breve distruggeranno in massima parte le specie fitofaghe, rendendo così un grande servizio alle piante che servirono loro di cibo, ma questo servizio sarà del tutto passeggiero; imperocchè, distrutte le specie fitofaghe, le specie carnivore dovranno emigrare o perire in massima parte per mancanza di nutrimento. Inoltre sarà quasi impossibile che alla strage non sfugga un qualche individuo delle specie fitofaghe. Or bene questi pochi individui rimasti, in grazia alla straordinaria fecondità degli insetti, arranno in breve ripopolato di specie fitofaghe il lungo; le specie carnivore essendo pel momento molto scarse, le fitofaghe piglieranno un grande sviluppo, ed in breve le piante, che servono loro di nutrimento, verranno a soffrire grandemente ed anche periranno. Alle specie fitofaghe verrà cosi a mancare il nutrimento e quindi anch'esse dovranno in massima parte perire; inoltre, il grande numero delle specie fitofaghe avrà fornito largo cibo alle specie carnivore, le quali avranno ricominciato a crescere in numero ed alla loro volta ricomincieranno la distruzione delle fitofaghe e cosi di seguito.

L'equilibrio quindi fra le specie che di tratto in tratto per l'anormale sviluppo di una parte di esse appare turbato, viene naturalmente stabilito senza che l'uomo vi entri per nulla.

Da ciò che abbiamo detto possiamo concludere che mancando le specie carnivore in una data località le specie fitofaghe non si moltiplicano indefinitamente e che le specie carnivore non sono indispensabili per frenare lo sviluppo delle specie fitofaghe. 
Ciò che ho detto intorno alle specie di insetti carnivore e fitofaghe lo possiamo ripetere applicandolo agli insetti in generale ed agli uccelli.

Gli insetti lıanno per nemici, fra gli animali delle altre classi, molti uccelli, micro-mammiferi, rettili, pesci, ecc. i quali fanno degli insetti loro nutrimento. Or bene, considerando di nuovo quel terreno incolto di cui ho già parlato, noi vi troviamo varie sorta di uccelli, di piccoli mammiferi insettivori, toporagni, ecc., varie sorta di rettili i quali tutti si nutrono di insetti. Supponiamo che il numero degli uccelli diminuisca. Che cosa succederà allora? A primo aspetto parrebbe, ed è ció che molti sostengono, anche al giorno d'oggi a torto, che gli insetti dovessero aumentar enormemente in numero. Or ció non avviene, aumenta invece il numero dei rettili, dei toporagni, ai quali è disponibile cosi maggiore copia di nutrimento. Se invece diminuissero i rettili ed i toporagni, aumenterebbero gli uccelli.

Supponiamo ora che gli insetti vengano per qualche causa speciale qualunque a scarseggiare, secondo sempre le idee dei sostenitori degli uccelli a distruggitori degli insetti, il numero degli uccelli e degli altri animali insettivori in quella località dovrebbe essere grande; ora ciò non è, mancando gli insetti, vengono pure a mancare gli uccelli, i toporagni ed i rettili, e ciò perchè viene loro a mancare il nutrimento. Una prova di quanto ho esposto la possiamo avere nel contorno stesso della nostra città. Basta che noi ci portiamo ad esempio in - Vanchiglia alla foce della Dora o della Stura, presso il R. Parco, per convincerci della esattezza dei fatti esposti. Le localitá sopra menzionate erano, una ventina d'anni fa, ricchissime di vegetazione arborea ed erbacea, grandissimo era pure il numero degli insetti che vi si trovavano, ed egualmente grande il numero degli uccelli, oggi invece, pel rovinoso deboscamento fatto lungo le rive della Dora e della Stura quelle località vennero ricoperte dalle piene dei fiumi di arida sabbia, la vegetazione ha diminuito molto, gli insetti sono pure enormemente diminuiti, e gli uccelli sono quasi al tutto scomparsi.

L'Agricoltura stessa, d'altra parte, esercita sugli insetti ed in generale sugli animali di una data località una grandissima influenza. 
Per spiegare più chiaramente questo concetto, torniamo al terreno incolto da me ripetutamente citato. Supponiamo che esso venga coltivato. L'agricoltore strappando le piante che ingombrano il terreno e sconvolgendo il terreno stesso coll'aratro distrugge un grande numero di specie di insetti, che o viverano sulle piante strappate, o compivano la loro metamorfosi nel terreno stesso.

In seguito alla seminagione una o due sorta di piante piglieranno il posto delle molte che l'agricoltore ha strappato. Poche pure saranno le specie di insetti che rimarranno, e saranno quelle precisamente che si nutrono delle piante seminate; ma queste essendo in grande numero, in breve il numero degli insetti crescerà pure e potrà crescere tanto da riuscire nocivo alle piante stesse.

Ciò non vuol dire tuttavia, come si crede in generale, che il numero degli insetti sia cresciuto. Prima della coltura della località citata vi erano molte sorta di insetti, oggi invece dopo la coltura le specie sono diminuite in numero, invece è cresciuto il numero degli individui appartenenti alle specie che rimangono.

L'agricoltura d'altra parte, è pure nociva allo sviluppo degli uccelli, distruggendo i boschi e i luoghi incolti coperti di cespugli.

Possiamo quindi conchiudere :

$1^{\circ}$ Che a superficie eguale, il numero totale d'insetti è eguale e nel luogo col tivato e nel luogo incolto, essendo eguale la quantità di cibo;

$2^{\circ}$ Che nel luogo incolto sono numerose le specie, poco numerosi gli individui che loro appartengono;

$3^{\circ}$ Che nella località coltivata sono poche le specie ed invece molto numerosi gli individui che loro appartengono;

$4^{\circ}$ L'agricoltura è contraria allo sviluppo non solo degli insetti, ma anche degli uccelli, dei rettili, dei piccoli mammiferi, e tende a favorire solo lo sviluppo di certe specie di animali.

Per ciò che riguarda gli insetti l'agricoltura ha un potentissimo ausiliare in una parte degli. insetti stessi. Oltre ai carnivori di cui ho già detto, vi ha un numero grandissimo di insettucci piccolissimi ed elegantissimamente coloriti, appartenenti in massima parte all'ordine degli imenotteri, i quali 
in svariate guise vivono alle spese di un numero grande di

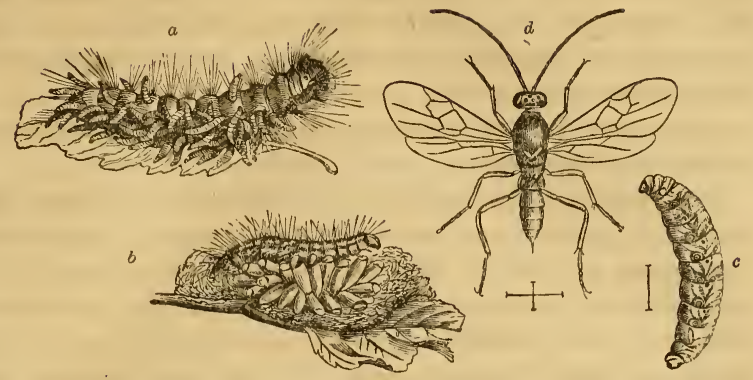

Fig. 1. - a Bruco della Cavolaia, dal quale escono le larve dell'Icneumone. $b$ Ninfa dell'Icneumone. - c Larva. $-d$ Insetto perfetto.

altri insetti attaccandoli in tutti i loro stadi di sviluppo dall'uovo all'insetto perfetto (1).

(1) Citerò, a mo' d'esempio, il catalogo delle specie parassite di una farfalla comunissima fra noi, del Bombice del salice, valendomi dell'opera del Rondani ("Degli insetti nocivi e dei loro parassiti, enumerazione con note ». Boll. Soc. Ent. Ital., vol. V, p. 4, 1873).

Musc. Doria concinnata. Mgn.

- Exorista noctuarum. Derv.

- Masicera sylvatica. Fall.

- Tachina larvarum.

- $\gg$ rustica. Fall.

Vesp. Bracon circumscriptus. Wsml.

- » pallidus. Gr.

- Campoplex assimilis. Gr.

- Cryptus analis. Grd.

- Mesochorus ater. Pitz.

- $\quad$ splendidulus. Gr.

- Pezomachus terebrator. Frst.

- Perilitus fasciatus. Hrtg.

- Pimpla instigator. Fb.

- » stercorator. Fb.

- Pteromalus eucerus. Rx.

- $\quad$ Halidayanus. Ritz.

- Rogas praerogator. Linn.

- Teleas punctulatus. Rtx.

- \ falenarum. Nees. 
Gli insetti parassiti servono moltissimo a frenare lo sviluppo degli altri insetti. È stato osservato in qualche caso che sopra dieci bozzoli di una farfallina Heterogynis penella $H$., nove furono invasi dall'insetto parassita che impedi lo sviluppo della farfalla stessa (1).

Anche pei parassiti è vera tuttavia la legge regolatrice dell'equilibrio fra le specie animali che ho sopra citato.

Ammettiamo finalmente per un momento che veramente gli uccelli e gli altri animali insettivori riescono realmente a distruggere gli insetti. Gli uccelli mangeranno gli insetti che loro sarà dato trovare senza fare distinzione fra specie carnivore e specie fitofaghe, senza fare distinzione fra parassita e non parassita, e quindi mentre da una parte saranno utili distruggendo le specie fitofaghe, dall'altra parte saranno dannosi distruggendo le specie carnivore ed i parassiti.

Supponendo anche che in una località ricca di insetti e di uccelli quest'ultimi crescano in grande numero e distruggano in un determinato periodo di tempo tutti gli insetti di quella località, che ne avverrà? Indipendentemente dalla mancanza di cibo che farà ridurre il numero degli uccelli insettivori, questi, cresciuti in numero, faranno alla lor volta aumentare il numero degli uccelli rapaci a cui servono di preda, i quali in breve li distruggeranno.

Da quanto ho detto sopra credo di poter conchiudere:

$1^{\circ}$ Che non ha molta importanza per l'agricoltura l'aumento o la diminuzione degli uccelli insettivori;

$2^{\circ}$ Affinchè realmente gli uccelli possano essere utili, bisogna che in un momento dato si trovino in quantità tale da distruggere in poco tempo tutti gli insetti, salvo poi a morire essi stessi di fame poco dopo.

Alcuni agenti esterni, dirò in ultimo, hanno pure grande influenza sullo sviluppo degli insetti. I grandi freddi, ed in generale le grandi variazioni di temperatura sono molto meno nocive agli insetti di quello che in generale non si creda, imperocchè le uova, le larve, le crisalidi e gli stessi insetti

(1) V. Ghiliani, «Ancora degli uccelli insettivori e degli insetti parassiti » Am. R. Accad. d'Agric. di Torino, vol. XVI, 1873. 
allo stato adulto, hanno una grandissima resistenza vitale (1).

Le grandi pioggie invece, le inondazioni, sono agenti che distruggono una grande quantità d'insetti. Ma in questo caso il rimedio, come facilmente si comprende, è peggiore del male.

Conchiuderò invocando di nuovo il concorso degli agricoltori, i quali soli colla diretta e diligente osservazione dei fatti, con esperienze diligentemente condotte possono portare qualche luce nella intricata questione del rapporto fra gli insetti e l'agricoltura.

\section{Bibliografia.}

Ratzeburg. - Die Forstinsecten oder Abbildung und Beschreibung der in den Waeldern Preussens, ete. Berlin.

J. Cur ris. - Farm. insects being the. nat. histor. and. economy. of the ins. injurius to the field crops of Great Britain, etc. London, 1860.

A. Dubors. - Traité d'entomologie horticole. Bull. de la fédération des sociétés d'horticulture de Belgique.

Borspuval. - Essai d'entomologie horticole. Paris, 1867.

W. T. HARris. - A Treatise on some of the insects injurious to vegetation. Boston, 1862.

Rıccr. - Memoria sugl'insetti più perniciosi al dipartimento del Metauro. Ancona, 1810.

Byle-Barelle. - Insetti nocivi all'uomo. Milano, 1824.

Passerini. - Molti lavori intorno a varie specie d'insetti nocivi inserti negli Atti della Accad. dei Georgofili nel Giornale Agrario Toscano e nei nuovi Annati delle Scienze nat. di Bologna.

C. Rondani. - Gli uccelli e gl'insetti dannosi all'agricoltura. Boll. del Comizio Agrario Parmense, 1868.

(1) Le uova della Sericaria mori L.resistettero, secondo lo Spallanzani, senza perire al freddo di $-50^{\circ}$. Vari bruchi osservati da Ross nelle regioni polari ripresero vita dopo una congelazione a $-42^{\circ}$, ripetuta quattro volte. Boisduval afferma pure che parecchie crisalidi non perirono sebbene si fossero agghiacciate. II Nicolet dice pure che una Podura stata congelata riprese vita. 
V. Ghuliañ. - Alcuni cenni sugli uccelli insettivi e sugli insetti parassiti. Ann. R. Accad. d'Agric. di Torino, 1871.

- Considerazioni sopra l'utilità dell' Entomologia e sua applicazione all' agricoltura. Giornale d'Agricoltura pratica, 1856.

- Insetti nocivi e legge sullo sbrucolamento. Ann. della R. Accad. d'Agric. di Torino, 1873, vol. XVI.

GENÈ. - Saggio sugli insetti più dannosi all'agricoltura, ecc. Bibliot. agraria. Milano, 1827.

- Istruzione sugli insetti più dannosi all'agricoltura nei Regii Stati, ecc. Torino, 1840.

G. SABBIONI. - Gli uccelli e gli insetti in rapporto coll'agricoltura. Giornale di Agricoltura del Regno d'Italia. Anno VII, 1870, vol. XIV.

E. De Betтa. - Degli Insetti nocivi all'Agricoltura, ecc., Atti Accad. d'Agricoltura, ecc. di Verona, 1865.

Apelle Der. - Insetti dannosi alle viti in Italia. Annali di viticoltura, ecc., in Italia. Milano, 1873.

Targion Tozzerti. - Relazione intorno ai lavori della Stazione e Acc. Agraria di Firenze. Ministero dell'Interno. Annali d' Agricoltura, 1878. 


\section{III. \\ ORIGINE DEGLI INSETTI}

Il Cuvier ed il Baer raggruppavano tutti gli animali intorno a quattro grandi tipi o piani generali di struttura.

A questa conclusione il Cuvier era giunto, studiando la struttura interna degli animali, il Baer studiando invece il loro modo di sviluppo.

Nella tavola seguente sono messe a confronto (per ciò che riguarda gli insetti) le classificazioni generali degli animali di Linneo, di Cuvier e quella recentissima, e che noi seguiremo, del Claus.

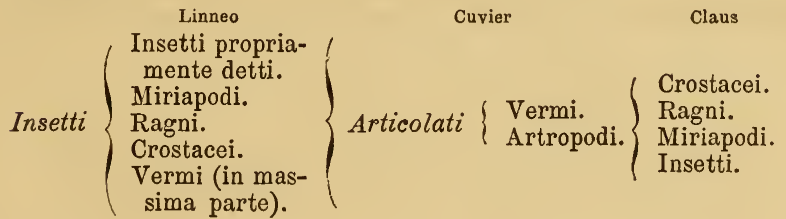

Modernamente il numero dei tipi in cui si intende diviso il regno animale è molto maggiore di quello che non ammettesse il Cuvier. Gli Articolati del Cuvier vennero divisi in due, nei vermi e negli artropodi, che comprendono i crostacei, i ragni, i miriapodi propriamente detti e gli esapodi.

I nuovi studi inoltre fatti intorno allo sviluppo degli animali e le nuove scoperte fatte nel campo della paleontologia hanno concesso ai moderni naturalisti non solo di stabilire la successione delle forme animali nel tempo, ma eziandio di stabilire in una maniera più o meno sicura la derivazione delle varie forme le une dalle altre. In una parola hanno fornito i mezzi 
per costrurre ciò che si chiama l'albero genealogico del regno animale (1).

Il tipo degli artropodi, come viene inteso oggigiorno, comprende:

$1^{\circ}$ Gli insetti propriamente detti provvisti di tre paia di zampe ;

$2^{\circ}$ I ragni aventi quattro paia di zampe;

$3^{\circ}$ I miriapodi provvisti di un grande numero di paia di zampe ;

$4^{\circ}$ I crostacei aventi un numero vario di paia di zampe, numero che tuttavia è inferiore a quello dei miriapodi.

Noi possiamo farci un'idea chiara del piano di struttura di un artropodo pensando ad una serie di animali identici saldati fra loro in linea retta, ciascuno costituente un segmento o zoonito, e ciascuno avente in sè gli organi della vita vegetativa e della vita animale.

Questo modo di intendere la struttura generale degli artropodi è applicabile pure agli articolati di Cuvier, e quindi anche ai vermi; nei quali anzi la cosa appare forse più evidente che non negli insetti.

Risalendo dalle forme più semplici dei vermi fino alle forme più complesse degli insetti, si vede che a poco a poco ciascun

(1)

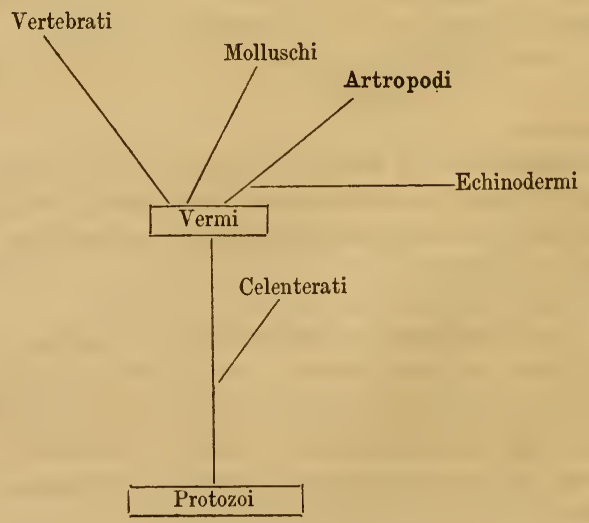


segmento tende a compiere un lavoro fisiologico distinto e l'omologia dei segmenti viene scomparendo. La divisione del lavoro fisiologico si fa sempre più evidente, e raggiunge il massimo suo grado negli insetti allo stato perfetto.

Il carattere più importante che distingue gli artropodi dai vermi consiste nella presenza sopra una parte degli anelli costituenti il corpo dei primi di appendici articolate, varie in numero, che servono alla locomozione. Inoltre, mentre nei vermi la locomozione si fa mediante il successivo spostarsi dei segmenti del corpo, e con movimenti di ondulazione del corpo intiero, negli artropodi questa funzione è localizzata in appendici speciali, e quindi, secondo la legge della divisione del lavoro fisiologico, essa si compie in un modo più perfetto.

E tuttavia molto probabile che gli artropodi abbiano avuto origine dai vermi articolati, e quantunque le zampe siano negli artropodi divise in articoli, quantunque il processo di differenziazione delle grandi divisioni del corpo sia molto più spiccato negli artropodi che non nei vermi, questi ultimi hanno tuttavia comune coi primi la forma del sistema nervoso, vale a dire un anello ed una catena gangliare ventrale, ed altre particolarità le quali, sebbene meno

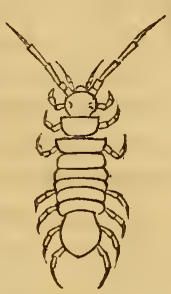

Fig. 2. importanti, servono nondimeno a provare sempre più la derivazione degli artropodi dai vermi.

Considerando inoltre l'intero tipo degli artropodi si vede chiaramente che essi sono animali con simmetria bilaterale molto evidente (1).

Esaminando il modo di sviluppo degli insetti e soprattutto il modo di sviluppo dello invoglio duro esterno che, come vedremo, piglia il nome di dermascheletro, noi possiamo farci un'idea

(1) Si ammetterebbe pure da qualche autore un altro genere di simmetria. Esisterebbe cioè una simmetria secondo un piano perpendicolare al primo sopracitato, una simmetria anteroposteriore. Le antenne e le altre parti dell'estremità anteriore sarebbero rappresentate nell'estremità posteriore da prolungamenti anali e dalle altre parti degli ultimi segmenti addominali. Quest'ultima maniera di simmetria è spiccata soprattutto in alcuni crostacei del genere Jara (Fig. 2). 
chiara dello sviluppo bilaterale, e acquistare una prova di più dell'esistenza della simmetria bilaterale negli insetti.

Consideriamo un segmento o zoonito. Nella maggiore sua semplicità, nei primordi del suo sviluppo il segmento si presenta in tutta la sua superficie formato da un tessuto omogeneo; una leggiera depressione trasversale, nella quale lo spessore del tessuto è un po' minore, indica solo il punto della sua separazione dagli altri segmenti.

Nel successivo svilupparsi del segmento, si cominciano a for-

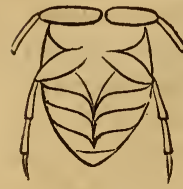

Fig. 3. mare delle areole più spesse e aventi l'aspetto dei punti di ossificazione dei vertebrati, questi spazi crescono poco a poco in grandezza e finiscono poi per occupare tutto il segmento.

Nel segmento giunto ad un certo punto del suo sviluppo si distinguono assai bene due archi, uno superiore, l'altro inferiore, che lasciano sui fianchi uno spazio membranoso più o meno esteso.

I due archi, il superiore o dorsale, l'inferiore o ventrale, risultano formati ciascuno da due parti simmetriche. La cosa

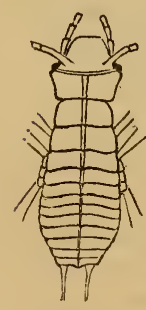

Fig. 4. ci appare evidentissima nelle larve di molti insetti. Le traccie tuttavia della primitiva divisione degli archi in due parti si conservano anche in qualche specie allo stato adulto.

Qualche caso di mostruosità mostra la cosa in un modo anche più evidente.

Il signor Verdiani Bandi ha osservato un caso di mostruosità molto raro e molto importante in un Rhizotrogus marginipes (1), in cui come si può scorgere dal disegno qui unito l'addome è diviso al metasterno in due lobi ben evidenti che si ricongiungono poi all'ultimo anello addominale (Fig. 3).

Nella larva della forficola auricularia, lo sviluppo caratteristico degli artropodi per mezzo della formazione primitiva di due metà identiche appare evidentissimo come si può osservare nella Fig. 4.

Ciò premesso, gli artropodi possono venire definiti così :

(1) Bollettino della Societì Entomologica Italiana, anno IX, p. 220. 
Animali con simmetria bilaterale, con corpo composto di anelli eteronimi aventi organi della locomozione articolati, provvisti di un cervello e d'una catena gangliare ventrale.

Negli insetti il di cui nome, dirò di passaggio, significa animale dal corpo formato da segmenti uniti fra loro (corpus intersectum); il corpo è diviso in un numero vario di segmenti, zooniti o metameri come si vogliono chiamare, in cui la divisione del lavoro fisiologico è stata portata molto innanzi. Noi troviamo già infatti il corpo degli insetti allo stato adulto, diviso in tre parti molto ben evidenti che compiono ad uffici speciali; queste parti sono: il capo, il torace e l'addome.

Ho detto nell'insetto giunto allo stato perfetto, imperocchè nell'insetto allo stato di larva in molti casi la testa solo è distinta dalle altre parti, e il resto del corpo è costituito da un numero più o meno grande di zooniti o metameri omonimi, senza che in essi si scorgano tracce nè di zampe, nè di divisioni indicanti il torace o l'addome (Fig. 5).

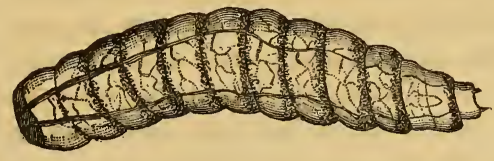

Fig. 5. Calliphora vomitoria.

Questo fatto è molto importante perchè viene a confermare una volta di più l'origine probabile degli articolati, e quindi anche degli insetti, dai vermi.

Ciascuna delle grandi divisioni del corpo degli insetti risulta dall'unione di un dato numero di segmenti o zooniti. Intorno al numero dei segmenti che debbono essere assegnati a ciascuna parte, non tutti i naturalisti vanno d'accordo. In generale si ammette che il capo risulti dalla fusione di quattro segmenti, tenendo conto delle quattro paia di appendici che, come vedremo, sono portate dal capo. Il torace è formato di 3 segmenti, di 9 o 10 , ed anche di 11 l'addome.

Ai segmenti costituenti il corpo degli insetti, vengono, come ho già detto, ad inserirsi numerose appendici.

Nel capo noi abbiamo nella parte superiore ed anteriore un paio di antenne, e un po' più in basso tre paia di appendici speciali che costituiscono gli organi masticatori. 
Il torace porta gli organi della locomozione. Questi possono essere di due sorta secondochè servono al camminare, al saltare e al nuotare, oppure al volo: nel primo caso pigliano il nome di zampe, nel secondo quello di ali.

Le zampe dipendono nel torace dagli archi inferiori dei zooniti, o metameri, le ali invece dagli archi superiori.

Le zampe sono in numero di tre paia.

Le ali sono in numero di due paia, uno inserto sul mesotorace e l'altro sul metatorace. Il protorace non porta ali.

Le ali negli insetti si trovano allo stato adulto, o in stadi di sviluppo molto vicini allo stato adulto, e sono certamente uno dei caratteri più importanti, e che determinano la superiorità degli insetti sugli altri artropodi. Esse tuttavia non bastano a caratterizzare gli insetti, imperocchè in molti, in forza di metamorfosi regressive, le ali mancano al tutto, e d'altra parte, in altri artropodi non insetti, ad esempio nei ragni, vediamo una tendenza al formarsi di organi destinati al volo (1).

L'addome non porta mai appendici articolate locomotrici negli insetti adulti (nelle larve vi hanno in molti casi appendici articolate, che pigliano il nome di false zampe, anche sulla parte inferiore dei segmenti addominali) meno un caso rarissimo che si osserva nelle specie del genere Japyx (Tisanuri) in cui i segmenti dell'addome presentano zampe rudimentali.

Nella figura 6 sono rappresentati schematicamente tre zooniti del corpo dell'insetto, nei quali si può vedere quale sia la posizione e il rapporto dei vari sistemi di organi fra loro (Fig. 6).

Gli insetti possono, tenendo conto di quanto abbiamo detto, venir definiti cosi :

Artropodi col corpo diviso in tre parti principali, capo, torace ed addome. Il capo porta le antenne e gli altri organi dei sensi. Il torace porta gli organi della locomozione (zampe, ali), e risulta costituito di tre segmenti ben distinti. $L$ 'addome contiene gli organi della riproduzione e consta in generale di dieci segmenti piu o meno separati fra loro e più o meno soiluppati.

(1) "Cambridge. On some new Genera and Species of Araneidea". Annals and Mag. of Nat. Hist. Vol. XIV, 1874, pag. 178. 
Definiti cosi gli insetti, cerchiamo quale sia la loro origine probabile e in che tempo questa origine abbia avuto luogo.

Le quattro classi in cui noi abbiamo detto dividersi gli artropodi, vale a dire i crostacei, i miriapodi, gli aracnidi e gli
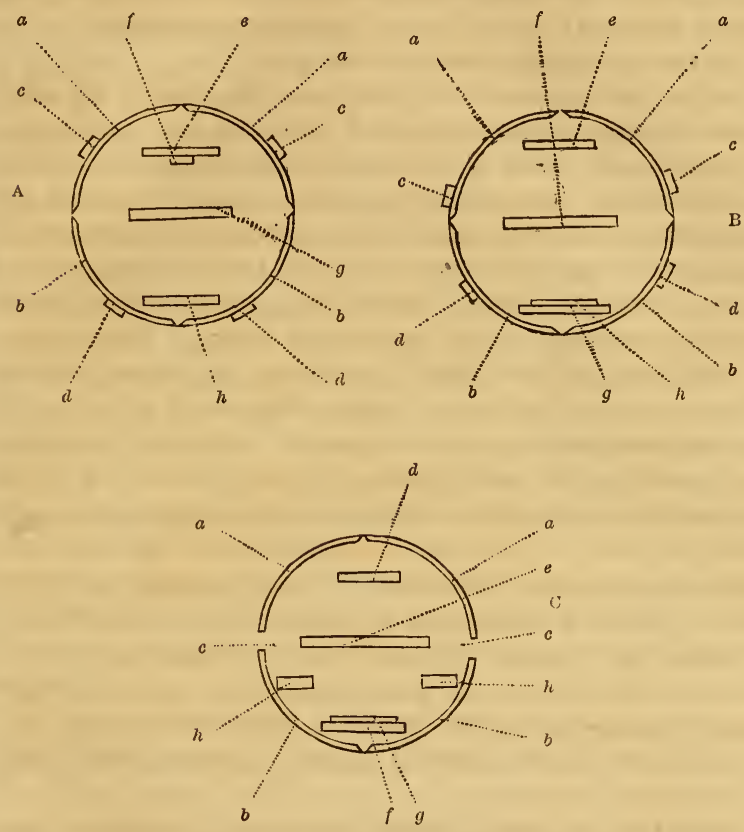

Fig. 6.

A. Capo, $a$ arco superiore, $b$ arco inferiore, $c$ antenne, $d$ appendici dell'apparato boccale, $e$ gangli cerebroidi, $f$ ganglio frontale, $g$ canal digerente, $h$ gangli sottoesofagei.

B. Torace. $a$ arco superiore, $b$ arco inferiore, $c$ ali, $d$ zampe, $e$ vaso dorsale, $f$ canal digerente, $g$ catena gangliare ventrale, $h$ grau simpatico.

C. Addome, $a$ arco superiore, $b$ arco inferiore, $c$ stimme, $d$ vaso dorsale, $e$ canal digerente, $f$ catena gangliare ventrale, $g$ gran simpatico, $h$ organi essenziali della riproduzione.

insetti possono venire unite in due grandi gruppi, secondochè gli animali che li costituiscono respirano l'aria libera, o l'aria sciolta nell'acqua, secondochè respirano per mezzo di trachee o per mezzo di branchie. Possiamo disporre la cosa così : 
Artropodi Branchiati / Crostacei (in massima parte).

$$
\text { » Tracheati }\left\{\begin{array}{l}
\text { Miriapodi. } \\
\text { Aracnidi. } \\
\text { Insetti. }
\end{array}\right.
$$

È senza dubbio probabile, dirò coll'Haeckel (1), che gli artropodi abbiano avuto origine dai vermi articolati: ma non sappiamo se l'intero tipo degli artropodi provenga da un solo ramo o da più rami di vermi articolati. Vi hanno ragioni per credere, ad esempio, che gli artropodi branchiati, e gli artropodi tracheati abbiano ciascuno avuto origine da un distinto ramo di vermi articolati. Considerando tuttavia come molto verosimile che i due grandi gruppi di artropodi sopra accennati discendano da un sol ramo di vermi articolati, siamo portati ad ammettere che gli insetti, gli aracnidi e i miriapodi si sono sviluppati posteriormente ai crostacei branchiati, ed anzi hanno avuto origine dal gruppo degli stessi crostacei branchiati.

Intricatissima si presenta pure la questione dell'origine degli insetti ed in generale dei tracheati dalle forme branchiate; io riferirò senz'altro a questo proposito l'opinione del Gegenbaur intorno a questo argomento, la quale, sebbene non sia ancora pienamente dimostrata, è tuttavia molto probabilmente conforme al vero:

«I rapporti che corrono fra le varie classi di tracheati, e fra queste ed i crostacei non possono vénir spiegate che coll'esame dei rapporti di queste classi col mezzo ambiente, tenendo calcolo in special modo degli organi respiratori. È cosa posta fuor d'ogni dubbio che la respirazione branchiale rappresenta il primo stadio e la respirazione tracheale uno stadio posteriore. Le condizioni anatomiche delle trachee, ed i loro rapporti colle aperture esterne delle stimme ci fanno supporre in esse uno stadio di sviluppo molto inoltrato, avvenuto in seguito ad una serie di trasformazioni. Le larve dei pseudonevrotteri (Efimere) ci presentano uno stadio analogo, quantunque transitorio, nelle loro branchie tracheali, le quali sono foggiate nello stesso tempo a mo' di membra. Noi tro-

(1) « Natürliche Schöpfungsgeschichte, gemeinverständliche wissenschaftliche Vorträge über lie Entwickelungslehre im allgemeinen und diejenige von Darwiu, Goethe und Lamarck im besonderen *. 1868, Berlino. 
viamo là un punto di contatto, e noi possiamo considerare queste parti che sono in rapporto colla vita acquatica, come lo stadio più semplice degli organi respiratori tracheali. Il fatto che questo sistema tubulare è primitivamente chiuso ci conduce a conchiudere che la prima forma degli organi precursori delle trachee dovette essere simile allo stadio che si osserva negli onisci..... Se dunque le forme chiamate dall'Haeckel protracheati che si rannodano ai crostacei branchiati, sono quelle che conducono alle forme provviste di branchie tracheali, noi dovremo considerare il gruppo in cui queste forme si trovano come il più vicino al ceppo primitivo. L'intiera classe degli insetti si accosta più al ceppo primitivo per mezzo dei pseudonevrotteri che non per mezzo degli aracnidi e dei miriapodi ».

Dai protracheati hanno avuto oxigine tre grandi serie di animali: i miriapodi, gli aracnidi e gli insetti. Questi ultimi sono legati ai protracheati per mezzo degli architteri (pseudonevrotteri), rappresentati al giorno d'oggi dalle efimere e dalle libellule, le quali presentano nel loro stadio larvale molti punti di contatto con certe forme larvali di crostacei branchiati.

Da un ramo degli architteri hanno avuto origine i neurotteri (Formicaleone, Friganea).

$\mathrm{Da}$ un altro ramo sono venuti gli ortotteri (Locusta, Grillo) e da questi i coleotteri (Carabo, Maggiolino, Coccinella), i quali differiscono dai primi essenzialmente per avere metamorfosi compiute.

Da un terzo ramo degli architteri, sono discesi gli emitteri (Afide, Notonecta, Pentatoma), e probabilmente da questi i ditteri (Mosca, Tafano).

Dai neurotteri poi hanno molto probabilmente avuto origine prima gli imenotteri (Ape, Formica), e poi i lepidotteri (Pieride, Bombice, Tignuola), i quali morfologicamente sono i più elevati fra tutti gli insetti, e sono anche quelli che sono apparsi gli ultimi alla superficie della terra.

Nel quadro seguente è disposto l'albero genealogico probabile degli insetti, e sono segnate le epoche geologiche in cui i singoli ordini degli insetti hanno presumibilmente cominciato ad apparire alla superficie della terra. 


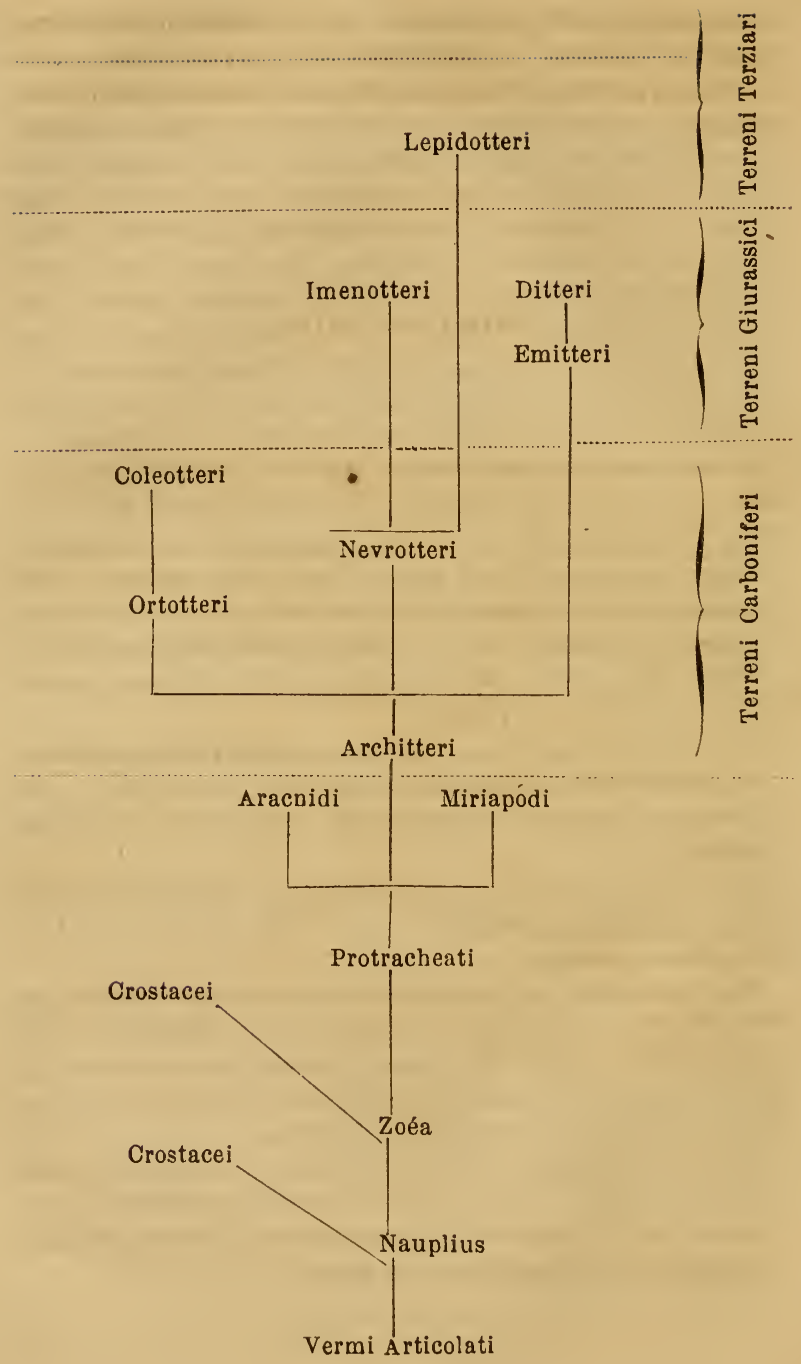


Riassumendo, noi possiamo dire che gli insetti hanno avuto origine dai crostacei, i quali alla lor volta derivano per mezzo delle due forme larvali Nauplius e Zoéa direttamente dai vermi articolati.

\section{Bibliografia.}

E. Haeckel. - Generelle Morphologie der Organismen. Allgemeine Grundzüge der Formenwissenschaft, mechanisch begründet durch die von $\mathrm{Ch}$. Darwin reformirte descendenztheorie. Berlin, 1866.

- Natürliche Schöpfungs geschichte; gemeinverständliche wissenschaftliche Vosträge über die Entwickelungslehre im allgemeinen, und diejenige von Darwin Goethe und Lamarck in besonderen. Berlin, 1868.

C. Gegenbaur. - Grundriss der vergleichenden Anatomie. Leipzig, zweite Auflage, 1878.

C. Claus. - Grundzüge der Zoologie. Marburg und Leipzig, 1876.

A. S. PACK ARD. - Guide to the Study of Insects. Salem., 1869.

L. Levckant. - Ueber die Morphologie unả die Verwandtschaftsverhältnisse der wirbellosen Thiere. Braunschweig, 1848.

S. C. Savigny. - Mémoires sur les animaux sans vertèbres. Parigi, 1816.

S. V. Audouln. - Sur les rapports naturels qui existent entre les appendices masticateurs, et locomoteurs des crustacés et ceux de même nature chez les insectes hexapodes et les Arachnides. Analise des traveaux de l'Acad. d. Scienc. en 1820, par G. Cuvier.

- Recherches anatomiqnes sur le thorax des animaux articulés et celui des insectes hexapodes en particulier. Annales d. Scienc. Natur., I, 1824.

Strrads-Dürckhe1м. - Considérations générales sur l'anatomie comparée des animaux articulés. Parigi, 1828. 


\title{
- IV. \\ LA SCELTA NATURALE
}

\author{
NEGLI INSETTI.
}

Molto spesso, passando in rivista il mondo degli insetti, cl vien fatto di imbatterci in fenomeni molto curiosi, in forme assai strane. Certe specie, ad esempio, per non citare che al-

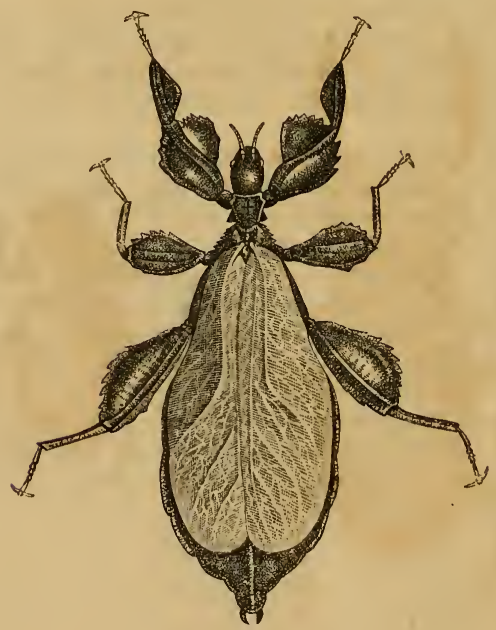

Fig. 7. Phyllium siccifolium.

cuni casi più spiccati (Phyllium) (Fig. 7), somigliano a foglie verdi, altre (Phasma, Bacillus) (Fig. 8) a ramoscelli secchi, altre a semi di piante, a granuli inorganici, a detriti vegetali, altre ancora (Cassida) a goccioline di rugiada. 
I due sessi inoltre negli insetti differiscono per lo più assai fra loro, sia nel colore, sia nella forma. Basterà ricordare a questo proposito il comune cervo volante in cui il maschio ha

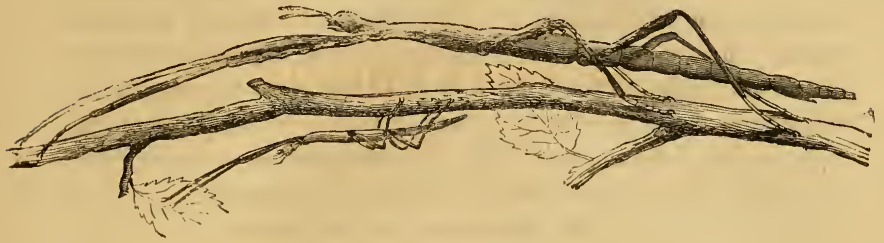

Fig. 8. Bacillus Rossii.

le mandibole enormemente sviluppate e che si prolungano anteriormente a mo' di corna, mentre la femmina è più piccola

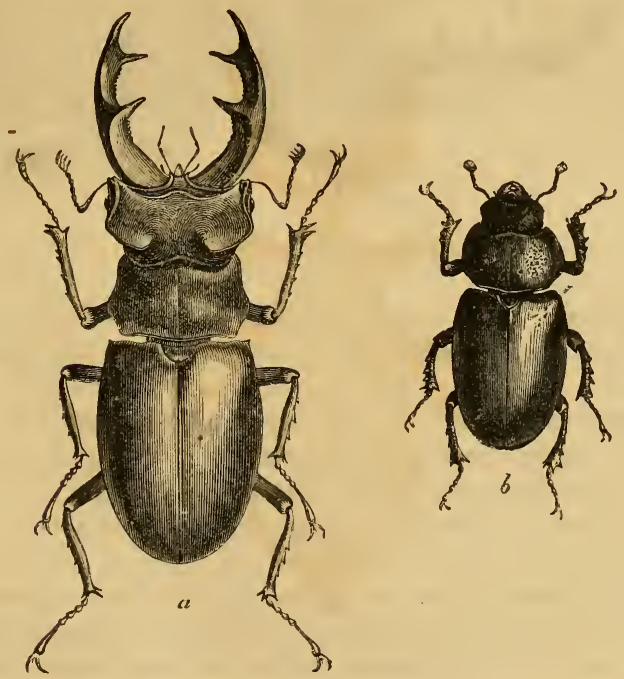

Fig. 9. Lucanus cervus. $-a$ maschio, $b$ femmina.

e le sue mandibole sono pochissimo sviluppate (Fig. 9). Molte specie poi di coleotteri esotici presentano differenze sessuali 
spiccatissime, tanto che i due sessi di una stessa specie vennero spesso classificati anche in generi diversi (Fig. 10).

Sorge spontanea innanzi a questi fatti in noi la domanda: Qual'è la ragione di queste strutture? come si sono originate? come si sono sviluppate? A questa domanda rimasta per tanto tempo senza soddisfacente risposta, venne risposto splendidamente da Carlo Darwin, il quale nel suo libro famoso intorno all'origine della specie, formolava fra le altre leggi regolatrici delle forme animali, due leggi importantissime, vale a dire:

$1^{\circ}$ La legge della selezione o scelta naturale;

$2^{\circ}$ La legge della selezione o scelta sessuale.

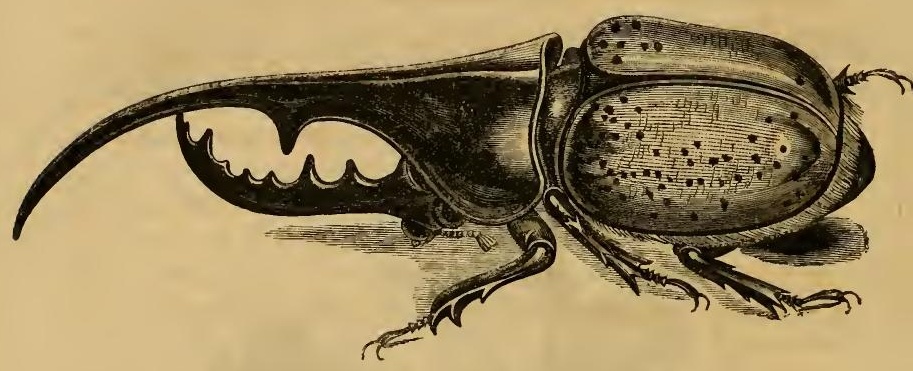

Fig. 10. Dinastes Hercules - máaschio.

Queste leggi si applicano assai bene al mondo degli insetti e ci danno una soddisfacente spiegazione dei fatti sopraccennati.

La lotta per la vita, la sopravvivenza degli individui più forti, sia fisicamente, sia moralmente, e la progressiva eliminazione delle specie e degli individui più deboli, ecco in poche parole in che cosa consista la legge che il Darwin chiama della scelta naturale.

Tutti gli esseri organizzati tendono a riprodursi rapidissimamente, secondo una progressione geometrica. Ciascuno degli esseri organizzati che durante la sua vita produce un numero più o meno grande di uova o di semi, secondochè consideriamo un animale o una pianta, deve in certi periodi della sua vita essere esposto a varie cause di distruzione; diversamente, per la legge delle progressioni geometriche, le specie verrebbero a 
moltiplicarsi in un numero cosi grande di individui che nessuna regione della terra potrebbe contenerli.

Ora siccome nasce un numero di individui superiore a quello che può vivere, deve esistere una lotta accannita, sia fra gli individui della stessa specie, sia fra gli individui di specie distinte, sia infine una lotta colle condizioni fisiche della vita stessa, e in questa lotta molti debbono perire. Si possono in una parola estendere all'intero mondo organico le leggi di Malthus intorno all'accrescimento delle popolazioni.

L'uomo, ad esempio, in cui la riproduzione è relativamente alla maggior parte degli animali lenta, può in soli 25 anni raddoppiare il numero dei suoi individui, e basterebbe qualche migliaio d'anni perchè non rimanesse più spazio alcuno sulla terra.

Il Darwin ha calcolato che una coppia sola di elefanti, che è fra tutti gli animali conosciuti quello che si riproduce più lentamente, potrebbe in soli 500 anni dare origine a quindici milioni di individui.

Se così è per gli animali superiori in cui la riproduzione è lenta, che cosa si dirà degli insetti, nei quali si producono in ciascuna riproduzione migliaia di uova, e in cui le riproduzioni si succedono rapidamente a brevi intervalli di tempo le une dalle altre?

Quasi tutti hanno un'idea della grande quantità di uova che una sola femmina del filugello può produrre. Suppongasi che il numero delle nova deposte sia solo di 200 e che la metà si sviluppi in maschi e l'altra metà in femmine. Queste ultime, supponiamo che diano ciascuna origine ad un numero di uova eguale a quello prodotto dalla prima femmina. Avremo alla fine dell'anno 20.000 uova. La metà di queste dia origine a femmine, le quali producano esse pure 200 uova ciascuna. In capo a tre sole generazioni, noi avremo 2.000.000 uova. Supponendo che le cose vadano sempre allo stesso modo, alla quarta generazione le uova saranno 200.000.000, alla sesta 2.000.000.000.000 (1). Si vede quindi, come dicemmo, che nel giro di pochi anni il numero degli individui provenienti da una sola coppia di insetti giungerebbe a una cifra enormemente grande.

(1) Una sola ape regina può in un sol giorno produrre oltre a 3000 uova. 
Ora in natura ciò non avviene punto. Ciascun individuo produce bensi un numero grande di uova, ma il numero degli individui adulti non varia in modo notevole, e si mantiene entro a certi limiti costanti.

Vi dev'essere naturalmente una ragione di questo fatto.

Io ho già accennato in un capitolo precedente, parlando dei rapporti dell'entomologia coll'agricoltura, ad una legge importantissima regolatrice dell' equilibrio delle specie animali fra loro, e del loro sviluppo, vale a dire, che lo sviluppo delle specie si fa in ragione diretta della quantità di nutrimento che è a loro disposizione.

Oltre alla quantità del nutrimento si deve anche por mente ad altre cause che influiscono sullo sviluppo degli animali.

Abbiamo da considerare in primo luogo l'influenza del clima. L'influenza del clima sullo sviluppo degli animali è molto grande, e il succedersi in periodi determinati di stagioni molto fredde e di stagioni molto secche è uno dei principali ostacoli al moltiplicarsi della maggior parte degli animali. Il clima inoltre agisce direttamente sugli individui delle varie specie, facendo perire i più deboli, indirettamente poi può pure favorirne, od impedirne lo sviluppo, avendo, come facilmente si comprende, una grande azione sulla quantità di cibo che è a disposizione degli individui stessi.

Il nutrimento e il clima sono certamente agenti importantissimi dell'equilibrio delle specie animali, ma non sono i soli che agiscono in questo senso; ad essi vengono ad aggiungersene altri risultanti dai mutui rapporti degli animali stessi fra loro. Tutti gli animali sono strettamente legati gli uni agli altri in quantochè per lo più gli uni servono di preda agli altri. Ne segue da ció che ciascuno lotta per ottenere il proprio mezzo di sostentamento: vi è lotta in una parola fra l'assassino e la sua vittima. Questa lotta può avvenire fra animali più o meno distanti fra loro nella scala zoologica, come è il caso di quasi tutti gli animali parassiti, oppure (e allora la lotta è ben più accanita), fra le specie appartenenti ad uno stesso genere, oppure àncora fra gli individui di una stessa specie; e quanto più le specie sono affini fra loro per struttura e per modo di vita, tanto più accanita e terribile è la lotta che ha luogo fra gli individui che ad esse appartengono. La ragione di questo ac- 
canimento sta in ciò, che in generale le specie affini frequentano la stessa località ed hanno bisogno dello stesso genere di nutrimento.

Conseguenza di questa lotta terribile fra due specie di uno stesso genere, è l'indebolimento ed anche l'eliminazione di una di esse e la prevalenza dell'altra. Conseguenza poi della lotta fra gli individui della stessa specie, è la modificazione e il perfezionamento della specie stessa colla sopravvivenza dei più forti, sia moralmente, sia fisicamente. Si possono trovare esempi di questa lotta in tutte le categorie di animali, io citerò due soli esempi tratti dagli insetti.

La Blatta germanica ha, sviluppandosi enormemente, cacciata dai luoghi dove essa abita la Ectobia Lapponica, mentre essa stessa cede a poco a poco il posto alla Periplaneta Orientalis specie molto più forte e robusta. La nostra Ape (come riferisce il Darwin nel suo libro Dell'Origine delle Specie) portata nell'Australia va via via distruggendo l'Ape indigena che è più piccola, più debole e sprovvista di pungiglione.

Nello stesso contorno di Torino (1) si possono osservare, sebbene in scala minore, fatti analoghi. Due specie di coleotteri carabici : il Gynandromorphus etruscus Quens, e l'Ophonus mendax Rossi, che una ventina d'anni fa erano comuni nel contorno di Torino, sono oggi rarissimi, la prima specie anzi è forse al tutto scomparsa. Un'altra specie invece pure di coleotteri carabici, l'Epomis circumscriptus Duft (specie non rara in Liguria e nell'Italia centrale e di cui al tempo del Bonelli, come risulta dai cataloghi del R. Museo Zoologico di Torino, se ne era preso qualche individuo presso il casamento delle vedove e nubili e che dal Bonelli in poi non era più stata trovata), pare si vada facendo da qualche anno più comune ed estenda la sua area di diffusione.

Il mondo quindi è un vasto campo di battaglia in cui gli gli esseri che lo abitano lottano continuamente per la loro esistenza e prosperità, sia cogli agenti esterni sia cogli organismi

(1) «L. Canerano. Di alcune variazioni avvenute nella Fauna Entomologica del contorno di Torino". Ann. della R.Accad. d'Agricol. di Torino, vol. XXI, 1878. 
di gruppi diversi che li assalgono, sia cogli individui della propria specie.

La lotta dell'esistenza è tanto più ardente ed accanita, quanto più sono simili le abitudini e gli istinti degli organismi che convivono in una determinata località.

È ciò che in ultima analisi accade nella società umana, in cui la concorrenza fra i vari individui è tanto più viva quanto più simili sono le loro aspirazioni.

Gli insetti, ed in generale tutti gli animali, hanno duopo, per lottare fra loro, di armi di offesa e di difesa. Queste armi esistono e sono svariatissime. Io citerò qualcuno dei più importanti mezzi di offesa e di difesa che si osservano negli insetti. Cominciamo dai mezzi di offesa.

Molti insetti hanno un apparato velenifero, il veleno del quale può in vari modi venir inoculato nel corpo dell'avversario. Per lo più vi ha un pungiglione ed un complesso di parti che, fatta la ferita, conducono in essa il liquido velenifero.

Gli organi veleniferi possono essere collocati presso alla bocca, come nei generi: Notonecta, Recluvius, Acanthia, ecc. fra i Rincoti, e nei generi: Culex, Simulium, Stomoxys, Haematobia, Glossinia, ecc. (1), fra i Ditteri; ovvero presso l'ano, come nei generi : Vespa, Apis, Bombus, Myrmica, ed in generale in tutta la divisione degli Imenotteri aculeati.

Le mandibole sono negli insetti in molti casi foggiate a mo' di organi potentissimi di offesa; hanno mandibole fortissime moltissimi Coleotteri, soprattutto carabici (generi: Megacephala, Cicindela, Anthia, Calosoma, Carabus, ecc.), e molti lamellicorni (generi: Lucanus, Chiasognathus, Lamprima, Pholidotus, Dorcus, Psalicerus, Platicerus, Macrodontia, Passandra). Nei Nevrotteri: Corydalis. Negli Ortotteri: Anostostoma.

Sul capo e sul torace di molti Coleotteri, soprattutto lamellicorni, si sviluppano corna molto robuste, le quali possono pure servire come armi di offesa. Generi : Megasoma, Megaceras, Chalcosoma, Scarabaeus.

(1) Glossinia morsitans. Westw (mosca Tsè-tsè). Questa specie è causa, a quanto raccontano $\mathbf{j}$ viaggiatori, di mortalità del bestiame nell'interno dell'Africa. 
Le zampe anteriori sono talvolta trasformate in armi di offesa molto formidabili.

Coleotteri, generi : Acrocinus. Ortotteri : Empusa, Mantis, Blepharis, Acanthops. Neurotteri : Mantispa. Rincoti : Nepa, Ranatra.

Possono pure essere annoverati come mezzi di offesa le somiglianze protettrici (somiglianze che, come dirò più sotto, sono anche nello stesso tempo mezzi efficacissimi di difesa) dei vari insetti, le quali concedono loro di appressarsi, non visti, alle loro vittime. Il genere Phyllium fra gli ortotteri, ha tutto l'aspetto di una foglia. Il genere Bacillus, pure fra gli ortotteri, ha l'apparenza di un ramo secco. Le casside, fra i coleotteri, sono appiattite e in generale di color verde, come le foglie sopra cui vivono. L'Harpalus ferrugineus che vive nei luoghi aridi e sabbiosi è di color giallo chiaro come l'arena. La larva del formicaleone è pure del colore della sabbia, ecc. (1).

Degli insetti gli uni usano delle armi (sopra enumerate) a viso aperto, altri invece accoppiano ad esse insidie più o meno ingegnose.

Sono nel primo caso, la maggior parte dei coleotteri carabici. Nel secondo alcuni fra i carabici stessi (genere Cicindela) (2). Fra i neurotteri, il formicaleone (3).

(1) Si noti tuttavia che nei Coleotteri carabici, questa categoria di mezzi di offesa è poco spiccata, e ciò forse perchè essi hanno già altre e potentissime armi nelle mandibole molto forti e robuste e nelle lunghe e veloci zampe. Sono invece molto sviluppate le somiglianze protettive nei fitofagi in generale, nei quali gli altri mezzi di oftesa sono più deboli.

Si noti ancora che in complesso le somiglianze protettrici e in una parola quel complesso di fenomeni che vanno compresi sotto il nome di fenomeni di mimismo si debbono piuttosto considerare come mezzi di difesa anzichè di offesa.

(2) La larva, ad esempio, della Cicindela campestris scava dei canaletti vertiticali nella sabbia, nei quali passa la maggior parte del suo tempo. Essa colloca il suo largo capo in prossimità dell'imboccatura in modo da chiuderla completamente. Quando un insetto passa sopra questa sorta di trabocchetto, essa ritrae bruscamente il capo, la vittima cade nella trappola e viene divorata.

(3) La larva del Mirmeleo formicarius Linn, scava nella sabbia mobile una sorta di imbuto, in fondo al quale essa si nasconde quasi al tutto sepolta nella sabbia. Quando un insetto, passando vicino all'imbuto a caso vi sdrucciola entro, essa gli getta contro col capo della sabbia per farlo rotolare al fondo, se ne impadronisce e succhia alla vittima le parti liquide e poscia ne rigetta la spoglia fuor dell'imbuto. 
Fra gli ortotteri, le mantidi (1).

Molto più vari e dirò anche più complessi sono i mezzi di difesa degli insetti.

Abbiamo anche qui come nei mezzi di offesa vari apparati ghiandolari destinati a produrre sostanze acri e corrodenti.

Nei coleotteri i generi Aptinus, Brachinus, Oxœna, Paussus hanno anzi un vero apparecchio esplodente, mediante il quale possono emettere violentemente, producendo uno scoppio più o meno intenso, una certa quantità di gas di odore disgustoso e di azione corrodente.

Un Rincoto, il Prionotus cristatus Am.-Serv., secondo il signor M. Dawis (citato da Kirby e Spence, nel loro trattato di entomologia), avrebbe la proprietà di essere elettrico (2).

Moltissimi insetti poi hanno secrezioni ed emanazioni fetide, le quali in qualche caso hanno un'azione corrodente più o meno spiccata.

I rincoti sono in gran parte puzzolenti. Spiccano fra gli altri per questa proprietà i generi Raphigaster, l'Acanthia, ecc. Nei coleotteri vi hanno molti carabici che hanno la stessa proprietà, generi: Carabus, Chlaenius, Panagaeus, Calosoma, ecc. Negli idrocantaridi osserviamo i generi Dytiscus e Gyrinus, che spandono quando vengono toccati, un liquido biancastro di odore irritante dall'articolazione della testa col torace.

Nei tenebrionidi osservansi il genere Blaps, che getta molto probabilmente dall'ano un umore nerastro e puzzolente, ed il genere Meloe, il quale lascia uscire dalle articolazioni delle zampe un liquido giallo che irrita fortemente la pelle.

Presentano fatti analoghi i generi Lytta, Mylabris, Coccinella, Megalopus, Timarcha, ecc. Nei lepidotteri le specie del genere Zygaena lasciano pure uscire dalle articolazioni

(1) Le Mantidi sono ortotteri eminentemente predatori che vivono sui cespugli. Questi insetti, scorta una preda, le si avvicinano con mille precauzioni, come farebbe un gatto verso un topolino, e quando le sono giunti a tiro rapidissimamente se ne impadroniscono colle zampe anteriori che sono enormemente sviluppate.

(2) Forse si tratta qui di un fatto analogo a quello che si osserva in alcuni miriapodi del genere Geophilus, tra i quali Linneo ne nominò uno Scolopendra electrica. La cosa tuttavia meriterebbe di essere confermata. 
delle gambe e delle antenne delle goccioline di un liquido speciale. Presentano inoltre emanazioni fetide fra gli ortotteri, la Periplaneta orientalis, ed alcune specie di Fasmidi, fra gli altri l'Anisomorpha bogotensis Goudot della Nuova Granata, la quale quando viene toccata spruzza a più di trenta centimetri di distanza un'umore lattiginoso molto acre. Fra i neurotteri vi è la Chrysopa merdaria e vari altri. Negli imenotteri osservasi l'Ophion merclarius Grav. e molte specie di formiche.

Alcuni insetti hanno pure emanazioni odorose, che all'uomo riescono piacevoli, ma che possono non esserlo a molti altri animali. Fra i coleotteri olezzano gradevolmente di muschio, alcune cicindele e qualche Pterostichus fra i carabici; il genere Necrophorus fra i palpicorni, ed il genere Aromia fra i longicorni.

I lepidotteri ci offrono la Sphinx convolvuli Linn. Ma in questo caso forse meglio che a difesa, l'odore serve come richiamo sessuale.

La fosforescenza di alcune parti può pure essere mezzo di difesa agli insetti. Credo tuttavia che la fosforescenza serva piuttosto come richiamo sessuale. Ne parlerò quindi un po' più a lungo trattando della scelta sessuale.

Trovano posto fra le armi di difesa i peli di varie larve di lepidotteri, peli i quali sono seghettati sui lati, e che nell'epoca delle mute della larva si staccano facilmente e penetrano producendo viva irritazione, nella pelle di chi tocca le larve o le spoglie recenti delle stesse.

Osservasi questo fatto nelle larve della Cnethocampa processionea Lim., della C. pityocampa Schiff., della Lithosia caniola Hubn., della Sitotroga cerealella Oliv.

$\grave{E}$ pure un mezzo efficacissimo di difesa la durezza più o meno grande dell'involucro esterno dell'insetto o dermascheletro.

Hanno un dermascheletro molto duro, fra i coleotteri quasi tutti i Buprestidi, generi Capnodis, Dicerea, ecc.; molti Tenebrioniti, generi Asida, Pimelia, Opatrum, ecc.; molti Lamellicorni, generi Cetonia, Scarabaeus, e molti Phanaeus, Copris, Onthophagus, Akis, ecc. e molti Corculionidi, generi Otiorynchus, Cleonus, Brachicerus, ecc. 
Fra gli Ortotteri quasi tutte le Forficole. Nei Rincoti i generi Nepa, Ranatra, ecc. Negli Imenotteri i generi Stilbum, Chrysis, ecc. Nei Lepidotteri il genere Sphinx.

In qualche caso inoltre, come ad esempio, nelle specie del genere Hispa fra i coleotteri ed in molti lepidotteri allo stato di bruco, il corpo è ricoperto da aculei e da spine più o meno robuste (1).

Veniamo ora a quei mezzi efficacissimi di difesa che sono le rassomiglianze protettrici, i fenomeni di mimismo.

L'uniformarsi del colore dell'animale al colore del mezzo in cui esso vive, costituisce uno dei mezzi più efficaci di difesa degli animali. Negli insetti noi troviamo quasi sempre il colore protettore più sviluppato in quelle specie le quali sono prive di altri mezzi di difesa.

Gli esempi dell'uniformarsi del colore dell'insetto col luogo in cui esso vive si incontrano nel mondo degli insetti ad ogni piè sospinto.

Io ho già parlato delle Casside e dell'Harpalus ferrugineus.

La Cicindela campestris, ad esempio, che vive per lo più nei luoghi erbosi, è di un bel colore verde, mentre la C. hybrida, la C. marittima, ecc., che vivono lungo le spiaggie del mare - nei luoghi aridi dell'interno delle terre, sono di un colore bruno-chiaro, più o meno dorato, ed è cosa assai difficile lo scorgerle quando sono posate sui ciottoli o sulla sabbia sotto i raggi cocenti del sole.

Lo stesso si dica per le specie del genere Omophron, e per quelle del genere Clivina, le quali vivono pure nei luoghi sabbiosi.

Gli Ortotteri poi ci offrono numerosissimi esempi di questa natura. La stessa specie ha colori diversi, secondochè abita luoghi ricchi di vegetazione od aridi.

In molti Rincoti, generi Pentatoma, Aphis, ecc., il colore del corpo è così somigliante a quello della foglia o del ramo su cui vivono che è cosa difficilissima poterli scorgere.

(1) Si osserva questo fatto soprattutto in vari lepidotteri esotici, ad esempio nel bruco della Ceratocampa regalis, il quale è per questa ragione ingiustamente molto temuto dagli indigeni dell'America del Nord. 
Nei lepidotteri poi la distribuzione dei colori sulle ali delle specie notturne e delle specie diurne viene pure a provare lo stesso fatto.

Nei lepidotteri diurni i colori vivaci sono collocati sulla parte superiore delle quattro ali, mentre la parte inferiore ha colori sbiaditi e poco appariscenti. Nei notturni invece sono le ali superiori che hanno colori bruni e poco appariscenti, mentre invece sono ornate di vivaci colori le ali inferiori. Nei lepidotteri diurni l'animale, quando è in riposo, ripiega le ali delle quali allora non rimane visibile che la superficie inferiore. I colori di questa armonizzano con quelli delle piante su cui l'insetto suole vivere. Nei notturni le ali inferiori sono ricoperte allo stato di riposo dalle superiori, le quali hanno pure colori armonizzanti col mezzo in cui l'insetto suole trovarsi (1).

(1) Credo utile per dare un'idea più chiara della cosa di riferire le parole stesse che il Wallace ha detto nel suo libro intorno alla scelta naturale ed intorno ad una specie di lepidotteri delle Indie: «L'esempio più notevole e più evidente che io conosca di una rassomiglianza protettrice nelle farfalle è quello della Kallima inachis, comune nelle Indie e della specie alleata di Malesia la Kallima paralehta. La superficie superiore di questi insetti è splendida ed evidentissima; la loro mole è grande e sono adorni di una larga striscia aranciata sopra un fondo celeste scuro. La superficie inferiore è di tinte molto variabili; su cinquanta esemplari non ve ne sono due esattamente uguali, vi si osservano tutte le gradazioni del grigio, del bruno, del rosso che si trovano nelle foglie morte, secche 0 in decomposizione.

« La sommità delle ali superiori termina in punta acuta, forma comunissima nelle foglie degli alberi e degli arbusti tropicali, e le ali inferiori si prolungano in una coda stretta e corta. Fra questi due punti corre una linea curva e scura che riproduce perfettamente la nervatura mediana di una foglia e da essa partono da ambe le parti alcune linee oblique che imitano le nervature laterali. Questi segni sono più visibili esteriormente alla base delle ali e interiormente verso il mezzo e la sommità. È veramente cosa curiosissima l'osservare il modo col quale le strie marginali e trasversali proprie di questo gruppo si sono modificate al punto da imitare la disposizione delle nervature delle foglie. Ma ciò non è tutto. Noi troviamo ancora che le farfalle rassomigliano alle foglie in tutti i loro gradi di decomposizione, ammuffite, bucherellate, macchiate irregolarmente di nero dalla presenza di funghi microscopici. Queste rassomiglianze tuttavia tornerebbero inutili ove con esse non concordassero le abitudini dell'insetto; se esso si posasse sulle foglie o sui fiori, se tenesse le ali distesé o movesse il capo e le antenne come i suoi congeneri, a nulla servirebbe il suo travestimento; noi possiamo tuttavia congetturare, seguendo l'analogia, che in questo caso come in 
Oltre alle somiglianze protettrici or ora accennate, gli insetti ne offrono altre egualmente efficaci che essi acquistano scimmiottando, per dir cosi, altri insetti i quali per qualche particolarità di struttura hanno minor numero di nemici e più facilmente sfuggono ai pericoli.

Molto frequentemente studiando i vari ordini in cui vengono divisi gli insetti, si trovano, ad esempio, coleotteri che hanno in tutto l'aspetto di certe vespe (1), ortotteri che rassomigliano a coleotteri (2), lepidotteri (3) e ditteri (4) che hanno il facies di certi imenotteri e via dicendo. Ma non solo questi fatti si osservano fra i vari ordini degli insetti, ma eziandio fra gli

altri, i costumi dell'insetto sono tali da mettere a profitto il travestimento. Io stesso ho potuto raccogliere a Sumatra gran numero di Kallima paralehta e posso assicurare l'esattezza dei seguenti particolari: Queste farfalle che frequentano le foreste seche e volano rapidissimamente non si fermavano mai sopra un fiore od una foglia verde. Esse si perdevano d'occhio soventi sopra un cespuglio o un albero morto dal quale, ed anzi talvolta dal luogo stesso su cui io fissava lo sguardo, dopo lunghe ricerche infruttuose, le vedevo tutto ad un tratto slanciarsi per sparire nuovamente alla distanza di venti o trenta metri. Ho trovato una o due volte l'insetto in riposo e ho potuto constatare allora la perfetta sua rassomiglianza colle foglie secche. Egli si posa sopra un ramo quasi verticale, tiene le ali esattamente ravvicinate e ricoprenti colla loro base il capo e le antenne. Le piccole code delle ali posteriori toccano il ramo e formano il peduncolo della foglia che è sostenuta dagli uncini del paio di zampe mediane, le quali sono sottilissime e poco evidenti, il contorno irregolare delle ali offre l'aspetto di una foglia raggrinzata. Noi abbiamo adunque le dimensioni, il colore, la forma, le macchie e le abitudini che si concordano insieme per costituire un travestimento che si può dire perfetto; il numero degli individui che godono di questa protezione, attesta sufficientemente la sua efficacia *.

(1) L'Emus hirtus, ad esempio, uno dei più grossi brachelitri dei nostri paesi, si confonde facilmente con certe specie di Imenotteri del genere Bombus o di genere affini. È pure molto somigliante alle vespe il genere Molorchus.

(2) L'Hypocephalus armatus del Brasile, specie rarissima e che per varie particolarità della sua struttura non si sa ancora bene in quale famiglia di coleotteri si debba collocare.

Questa specie, come si può vedere dalla Fig. 11, ha moltissima rassomiglianza pel complesso della struttura colla comune grillotalpa. Sfortunatamente non si conosce nulla dei suoi costumi e quindi non si può dire se come la struttura del corpo anche i costumi siano analogi a quelli della grillotalpa.

(3) Genere Sesia.

(4) Genere Volucella. 
insetti appartenenti allo stesso ordine (1). Questi fatti conosciuti da lungo tempo, ma che non vennero mai presi in considerazione, sono stati soddisfacentemente spiegati dalla legge moderna della scelta naturale. È stato dimostrato, dirò col Wallace, che «questi fenomeni sono regolati da leggi fisse e definite, le quali-sono in stretto rapporto colla legge generale della sopravvivenza del più adatto e della conservazione delle razze più favorite nella lotta per la vita ».

Anche nella difesa gli insetti fanno uso di astuzie, alcune delle quali sono molto curiose.

Gli stafilini, ad esempio, fra i coleotteri, che sono affatto innocui, rialzano gli ultimi segmenti dell'addome (come fa lo

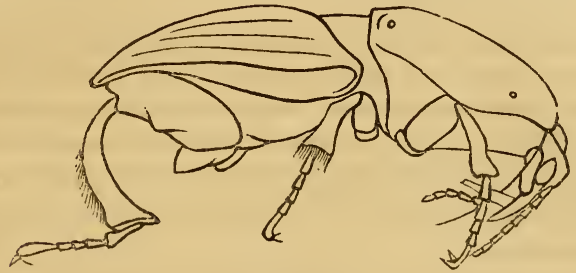

Fig. 11 .

scorpione) affinchè gli altri animali possano crederli armati di pungiglione.

Altri come i bruchi delle sfingi ricorrono a pose grottesche

(1) Citerò per dare un'idea chiara della cosa qualche esempio. La famiglia delle Eliconidi fra i Lepidotteri (famiglia che è ricchissimamente rappresentata nelle foreste dell'America meridionale) presenta forme molto belle per colori eleganti e per disegni svariati, la qual cosa non torna loro certamente di vantaggio. Esse tuttavia sono efficacissimamente protette da un odore fortissimo che tramandano. Per questa ragione molti uccelli le lasciano in pace. Or bene, il gruppo delle Eliconidi così efficacemente protette, viene imitato da altri generi di lepidotteri appartenenti a famiglie differenti dal genere Leptalis, ad esempio, fra le Pieridi, e dal gruppo delle Erycinidi, ecc. Le specie appartenenti a questi ultimi generi vengono confuse dagli uccelli insettivori e da altri animali pure insettivori, colle vere Eliconidi e quindi restono salve. Trovano esse così nella loro rassomiglianza colla specie più forte, un mezzo efficacissimo di difesa. Nei coleotteri un Longicorne, il Collyrodes Lacordairei ha precisamente la forma del genere Collyris, appartenente alla famiglia delle Cicindele. Il Doliops curculionoides ed altre specie affini, hanno molta rassomiglianza colle specie del genere Pachyrhynchus, pure appartenente alla famiglia dei Curculionidi. 
cercando di incuter spavento in questo modo ai loro nemici, oppure tentano di sfuggire ai loro nemici simulando la morte; ora avvoltolandosi a sfera come nei Coleotteri il genere Hypocyptus fra i Brachelitri, i generi Clambus ed Agathidium, nella famiglia delle Silfe e i generi Sphaeromorphus e $S y$ narmostes fra i Lamellicorni; negli Imenotteri i generi $\mathrm{He}$ dychrum, Stilbum, ecc.; negli Ortotteri le femmine attere delle specie del genere Perisphaeria nella famiglia delle Blatte; ora lasciandosi ad un tratto cadere a terra dalle foglie o dal tronco sopra cui stavano, e rimanendo immobili fra le erbe. Si osserva questo fatto principalmente in quasi tutti i generi appartenenti alle famiglie degli Isteridi, dei Birridi e dei $\mathrm{Bu}-$ prestidi fra i Coleotteri (l).

Accennerò finalmente ad un fatto singolarissimo che si osserva nella larva della Hydrophilus piceus Linn. La larva di questa specie che vive nelle nostre acque stagnanti, quando viene toccata schizza dall'ano un umore scuro che intorbida l'acqua, (un fatto analogo, come si sa, si osserva pure in vari cefalopodi) ed inoltre essa diventa floscia in modo da imitare perfettamente un corpo marcio.

Tutti i fatti, tutte le strutture sopra accennate sono il portato della scelta naturale. Il più piccolo grado di variazione, dirò col Wallace (2), delle specie, che noi consideriamo spesso come cosa puramente accidentale, anormale o troppo insignificante per meritare la nostra attenzione, è tuttavia il fondamento di tutte quelle analogie meravigliose che hanno tanta importanza nell'economia della natura.... « La rapidità delle riproduzioni, il continuarsi delle variazioni anche le più leggiere e la sopravvivenza dei più adatti e dei più forti, ecco le leggi che terranno sempre il mondo organizzato in armonia con se stesso e col mondo inorganico ».

(1) La stessa cosa, sebbene in grado un po' minore, si osserva pure in molte specie di Curculionidi, specialmente del genere Otiorinchus, e in qualche genere appartenente alla famiglia dei Crisomelini.

Fra noi è principalmente da notarsi fra le specie di Buprestidi che offrono quel singolare costume la Dicerca aenea Linn, la quale si lascia cadere dai tronchi lisci del Populus alba (sopra i quali si trova ordinariamente), solo che uno si avvicini ad una distanza di un dieci o quindici passi dal tronco stesso.

(2) Op. citata. 


\section{Bibliografia.}

C. DARWIN. - On the origin of species by means of Natural selection.

- The descent of man and selection in relation to sex.

G. Seidurzz. - Die Darwin'sche Theorie. Leipzig, 1875.

Weismans. - Studien zur descendenz Theorie, 1876.

0. Sснмирт. - Descendenzlehre und Darwinismus. Leipzig, 1873.

G. CAnestrixi. - La teoria dell'evoluzione esposta nei suoi fondamenti. Torino, 1877. 


\title{
V. \\ LA SCELTA SESSUALE
}

\author{
NEGLI INSETTI.
}

Scelta sessuale chiamo il Darwin la causa produttrice delle differenze che si trovano fra i sessi degli animali.

Rispetto a questa legge è necessario che noi anzitutto osserviamo:

$1^{\circ}$ Che la scelta sessuale agisce direttamente sui caratteri sessuali secondari, su quei caratteri cioè che differenziano i sessi all'infuori degli organi essenziali della riproduzione, vale a dire gli organi destinati a preparare gli ovuli nella femmina ed il liquido fecondante nel maschio.

$2^{\circ}$ Che i caratteri sessuali secondari si incontrano per lo più molto sviluppati solamente nei maschi.

$3^{\circ}$ Che il numero dei maschi è negli animali in generale superiore a quello delle femmine.

$4^{\circ}$ Che i maschi si sviluppano generalmente prima delle femmine, e sono prima di queste atti alla fecondazione.

Quest'ultima legge è principalmente applicabile agli insetti. La sproporzione numerica fra i sessi produce naturalmente la lotta fra i maschi pel possesso delle femmine.

Supponiamo che in una data specie di insetti i maschi siano al tutto somiglianti alle femmine, non presentino cioè nessun carattere sessuale secondario. Fra i maschi di questa specie, per la ragione sopradetta, vi sarà lotta accanita pel possesso delle femmine. In questa lotta avverrà ciò che io ho detto avvenire nella lotta fra gli individui di due specie diverse, sopravvivranno cioè quegli individui i quali saranno superiori agli altri per qualche particolarità di struttura o pel maggior 
sviluppo di qualche parte. Questi ultimi essendo i soli che possano dar opera alla riproduzione, accoppiandosi colle femmine, a misura che queste si sviluppano, daranno origine a maschi, nei quali quelle date particolarità di struttura che avevano concesso la vittoria ai loro progenitori saranno spiccatissime. A poco a poco l'altra sorta di maschi, quelli cioè più deboli, non potendo essi riprodursi o solo scarsamente, verranno a mancare. I maschi quindi saranno in un numero indeterminato di successive riproduzioni divenuti molto diversi dalle femmine, avranno cioè acquistato certi determinati caratteri sessuali secondari.

In questo modo si spiegano tutti quei caratteri sessuali secondari destinati ad accrescere la forza dei maschi. Ad esempio il grande sviluppo delle mandibole, delle zampe e delle ali.

Il caso ora citato di scelta sessuale è uno dei più semplici e la femmina vi ha poca azione.

Nella maggior parte dei casi tuttavia le cose si complicano e le femmine scelgono esse stesse i maschi e non solo scelgono i più forti, ma scelgono anche i più belli. In molte specie quindi la bellezza tiene luogo della forza. Di qui la ragione per cui nella massima parte degli insetti, ed in generale anche degli altri animali, i maschi sono più belli delle femmine. La bellezza dei maschi si è prodotta in essi nello stesso modo in cui ho detto essersi prodotto lo sviluppo delle mandibole, delle zampe, delle ali, ecc.; si è prodotta, cioè per mezzo di successive scelte da parte delle femmine, dei maschi che offrivano qualche carattere che per esse era bello ed elegante (1).

Gli effetti notevoli che la scelta naturale e la scelta sessuale hanno prodotto e vanno producendo tuttora negli animali, si spiegano colle leggi dell'eredità. Intorno ad esse io non dirò nulla, chè esse ci trascinerebbero troppo fuori del nostro campo. Chi desidera prendere conoscenza di queste leggi consulti le opere già ripetutamente citate del Darwin, del Wallace, dell'Haeckel e del Canestrini.

(1) Si consulti a questo riguardo l'opera del Darwis, L'Origine dell' uomo $e$ la scelta in rapporto col sesso. Traduzione italiana del prof. Michele Lessona. Torino, 1872, p. 185 e seg. 
Passiamo ora il pii rapidamente possibile in rassegna i principali caratteri sessuali secondari degli insetti.

I caratteri sessuali degli insetti si possono dividere in due grandi categorie, in quelli cioè che riguardano il colore ed in quelli che riguardano la forma.

A produrre i caratteri della prima categoria concorre molto probabilmente anche la scelta naturale e in qualche caso anzi pare sia essa sola che agisca. A produrre i caratteri della seconda categoria concorre invece quasi esclusivamente la scelta sessuale.

Citerò a questo proposito il brano seguente del Wallace (1): «Negli insetti, la femmina di cui il volo è più debole e che è ad un tempo più esposta e più importante del maschio ha bisogno di una protezione speciale. Per la quale cosa i colori delle femmine sono in generale più scuri e meno appariscenti che non quelli dell'altro sesso. Io attribuisco ció a questa e non a quella causa che Darwin ha chiamato selezione sessuale, e ciò perchè nei gruppi provveduti di una qualsiasi protezione che li dispensi dal nascondersi per sfuggire ai loro nemici, le differenze sessuali di colore o mancano o sono poco sviluppate, la qual cosa coll'ultima ipotesi sarebbe inesplicabile. In fatti, nelle Eliconidi e nelle Danaidi protette dal loro sgradevole odore, le femmine sono splendide ed appariscenti come i maschi e ne differiscono molto raramente. I due sessi sono pure quasi identici negli Imenotteri portatori di pungiglione. Nei Carabici, nelle Coccinelle, nei Chrysomelini, nei Teleforidi gli individui dei due sessi sono in egual grado splendidi e raramente diversi nel colore. I Curculionidi, che sono protetti dallo invoglio duro, sono brillanti nei due sessi ; infine i $\mathrm{Ce}$ tonidi ed i Buprestidi, che appaiono protetti dalle loro corazze dure e levigate, dai loro movimenti rapidi e dai loro singolari costumi, presentano poche differenze sessuali rispetto al colore; mentre la selezione sessuale si manifesta sovente con diverse strutture come corna, spine ed altre».

I casi in cui i due sessi si differenziano fra loro pel colore sono frequentissimi negli insetti ed abbondano specialmente nei

(1) Op. citata. 
lepidotteri. Sempre quando questa differenza esiste, il maschio è più brillantemente e più elegantemente colorito della femmina. Negli ortotteri raramente i due sessi sono diversi nel colore. Lo stesso si dica per i neurotteri.

Nei rincoti non sono rare, soprattutto negli emitteri, le differenze sessuali di colore.

Leggiere differenze di colore si osservano pure, ma non molto frequentemente, negli imenotteri e nei ditteri.

Più frequentemente invece che non nei ditteri e negli imenotteri il maschio differisce nei coleotteri dalla femmina pel colore.

Molto più numerosi e più vari sono i caratteri sessuali secondari che riguardano la forma. La piu importante delle differenze riguardanti la forma si è la mancanza, abbastanza frequente, in uno dei sessi degli organi del volo ed in qualche raro caso anche delle zampe.

Quando questa differenza sessuale si verifica è nella massima parte dei casi il maschio che è alato, ed è la femmina invece che è attera ed apoda (1). Troviamo esempi di questo fatto in quasi tutti gli ordini di insetti.

Nei coleotteri le femmine del genere Drilus sono attere, mentre i maschi sono alati.

Nei Lepidotteri le specie, ad esempio, del genere Heterogynis sono attere ed hanno zampe appena rudimentali, mentre ben sviluppate sono nel maschio le zampe e le ali.

Fra i Neurotteri le femmine degli Strepsitteri sono attere ed apode, mentre i maschi sono alati.

Molte specie di Rincoti presentano pure maschi alati e femmine attere.

I sessi negli insetti si differenziano fra loro, in generale, anche nella mole. Il maschio è ordinariamente più piccolo della femmina. In qualche caso tuttavia le cose vanno diversamente e il maschio è molto più grande della femmina.

Gli altri caratteri sessuali secondari riguardanti la forma, oltre

(1) Un Imenottero parassita forma un'eccezione alla regola, non avendo il maschio che ali rudimentali e non abbandonando mai la cella ove è nato, mentre la femmina ha ali ben sviluppate. Vedi a questo proposito DARwIN, Origine delPuomo. Traduzione italiana del prof. Michele Lessona, p. 197. 
a quelli or ora accennati, possono da noi venire divisi in due categorie, in quelli cioè che accrescono la forza dei maschi, ed in quelli che aumentano la loro bellezza.

Appartengono alla prima categoria, ad esempio, le mandibole, le zampe, e le ali molto sviluppate di molti maschi.

Hanno mandibole sviluppatissime fra i Coleotteri i maschi del Lucanus cerous, del Dorcus parallelepipedus e di molti altri Lamellicorni già citati nel capitolo precedente.

Il grande sviluppo delle zampe anteriori, di cui ho pure già detto nel capitolo precedente, è in molti casi proprio dei soli maschi.

I maschi di molte specie di insetti, soprattutto di coleotteri, hanno i tarsi delle zampe anteriori molto più robusti e sviluppati che non quelli delle femmine e sono tapezzati inferiormente da un grande numero di peli, i quali agiscono come ventose (1) e servono a tener ferma la femmina nell'atto dell'accoppiamento. Sono in questo caso quasi tutti i carabici e molti lamellicorni (2).

In altri casi (generi Hydrophilus, Dytiscus, ecc.) gli ultimi articoli delle zampe anteriori dei maschi subiscono una modificazione più profonda e si foggiano a mo' di due grosse ventose.

Nelle forficole fra gli Ortotteri, sono le appendici dell'apparato copulatore esterno che sono molto diverse nei due sessi. Nei maschi l'ultimo segmento addominale porta due appendici allungate e ricurve in modo da formare un robusto paio di tanaglie, colle quali l'insetto tiene ferma la femmina nell'atto dell'accoppiamento. Nella femmina le appendici dell'ultimo segmento dell'addome sono invece deboli e poco sviluppate.

In altri casi è la femmina che offre un qualche speciale carattere sessuale secondario. Le ali, ad esempio, della femmina del Ditisco comune e di altre specie di Idrocantari, hanno numerose e profonde solcature longitudinali, mentre quelle del maschio sono perfettamente liscie (3).

(1) Vedi più sotto al capitolo VI.

(2) Qualche volta (Geniates barbatus, ecc.) tal sorta di peli si trovano pure sul mento dei maschi e servono allo stesso uso.

(3) Anche questa struttura delle ali delle femmine favorisce l'accoppiamento. Probabilmente si ha qui un caso di scelta sessuale esercitata principalmente dal maschio. 
Le antenne, più sviluppate, in generale, nei maschi che non nelle femmine, sono forse di giovamento ai primi nella ricerca delle femmine stesse.

Svariatissime, ed in molti casi assai strane, sono le strutture che non servono ad altro molto probabilmente che ad accrescere la bellezza dei maschi.

Vengono in prima linea fra gli organi ornamentali le corna che fra i coleotteri ci presentano i maschi dei generi Megasoma, Megaceras, Scarabaeus, Copris, Oryctes, ecc.

Certi tubercoli poi, certe spine che si osservano sul corpo di molti maschi, servono pure molto probabilmente come organi ornamentali.

Mi rimane ancora, prima di lasciare la legge della scelta sessuale, da far parola di un'altra serie di caratteri sessuali secondari, molto importanti, ma che non entrano nelle categorie sopra accennate. Questi caratteri possono venir divisi in tre gruppi :

$1^{\circ}$ Secrezioni di odori speciali;

$2^{\circ}$ Fosforescenza;

$3^{\circ}$ Apparati musicali.

Molte delle secrezioni odorose di cui ho fatto parola nel capitolo precedente, oltre al servire come mezzo di difesa, servono pure come richiamo sessuale, soprattutto quelle che hanno odore di muschio, uno degli odori più sparsi nel regno animale e funzionante appunto come richiamo sessuale. In qualche caso è il maschio solo che possiede la proprietà di mandare un forte odore di ambra o di muschio come si osserva nella Sphinx convolvuli (1).

La fosforescenza di certe parti oltre al servire essa pure, come ho già detto, come mezzo di difesa spaventando vari nemici degli insetti, è pure un importante carattere sessuale secondario.

Molti sono gli insetti fosforescenti ; ricorderò i principali :

(1) Vedi a questo proposito P. Stefanelur: «Sull'odore di ambra o di muschio che tramanda la Sphinx convolvuli Linn ». Bull. Soc. Ent. Ital. Anno II, 1870, p. 280.

TARg10ni Tozzerri: "Sull'apparecchio che separa ed esala l'odore di muschio nel maschio della Sphinx convolvuli». Bull. Soc. Ent. Ital. Anno 1870, p. 358. 
Coleotteri : molti generi di Malacodermi, Lampyris, Phosphaenus, Luciola, ecc. Vari generi di Elateridi, Photophorus, Pyrophorus, ecc. Nei ditteri ha il capo fosforescente la Thyreophora cynophila Lat. Meig.

Nei Rincoti, in seguito ad un errore di Sibilla di Merian, si credette per molto tempo che il capo della Fulgora lanternaria fosse fosforescente. È oramai cosa posta fuori di ogni dubbio che la fosforescenza non esiste in permanenza nella testa. Si sospetta che questa sia fosforescente prima dell'accoppiamento nell'uno o nell'altro sesso.

Nei lepidotteri si sono detti fosforescenti gli occhi della Sphinx convolvuli Linn., i bruchi della Agrotis occulta Linn., e della Mamestra brassicae Linn.

Nel primo caso non si tratta di fosforescenza, ma bensì di luce riflessa. Nulla si vede nell'oscurità perfetta. Nel secondo caso non si tratta di un fatto costante, ma bensi di uno stato patologico dell'animale.

Chi desidera avere maggiori particolari intorno alla natura del fenomeno della fosforescenza ed intorno alla struttura dei vari organi che lo producono, consulti le opere seguenti:

Matreuccr. - Lezioni sui fenomeni fisico-chimici dei corpi viventi. Pisa.

Peters. - Ueber das Leucten der Lampyrís italica Mullers Archiv. 1841.

Korr,LIKER. - Ueber die Leuchtorgane von Lampyris Verhandl.

d. phys. med. Geseh. in Wurzb. T. 8. 1857.

Targionı 'Tozzettr. - Dell'organo che fa lume nella Lucciola, ecc.

Mem. Soc. Ital. Scienz. Nat., vol. I, 1865.

- Nuove osservazioni. Boll. Soc. Ent. Ital., anno II, 1870. Owzinnnikow. - Mém. de l'Acad. Imp. des Sc. de St-Pétersbourg, vol. II, 1868.

P. Panceri. - La luce degli occhi delle farfalle. Rendiconto della R. Acead. di Se. fisiche di Napoli, ottobre 1872.

Più numerosi forse che non i fosforescenti sono gli insetti capaci di produrre qualche suono. Ora gli apparati fonanti sono propri di un solo sesso, e in questo caso è il maschio che n'è fornito (1). Ora invece si trovano in tutti due i sessi.

(1) \& Sono felici, dice il poeta greco Xenarchus, i maschi delle cicale, perchè le loro mogli non hanno voce" . 
Nei Rincoti, come è noto a tutti, i maschi delle cicale producono nella buona stagione un suono acuto e stridente. Riferisco a questo proposito il brano seguente dell'Origine dell'uomo di Darwin: «Rispetto allo scopo di questa musica (parla del suono delle cicale) il dottor Hartman parlando della Cicada septemdecim degli Stati Uniti dice: "Si sentono ora (6 e 7 giugno 1851) i tamburi in ogni direzione. Credo che questi suoni siano gli inviti di nozze per parte dei maschi. Stando io ritto in mezzo ai fitti castagneti alti quanto me, dove centinaia di questi insetti mi attorniavano, osservai le femmine accorrere intorno ai maschi suonatori». Egli aggiunge: "In questa stagione (agosto 1868) un pero nano del mio giardino produsse una cinquantina circa di larve di C. pruinosa, ed osservai parecchie volte le femmine posare accanto al maschio mentre faceva risuonare le sue acute note ».

«Federico Müller mi scrive dal Brasile meridionale che ha sovente udito una lotta musicale fra due o tre maschi di cicale dotati di una voce singolarmente forte e posati ad una notevole distanza l'uno dall'altro. Appena il primo areva terminato il suo canto, il secondo cominciava immediatamente il suo; e dopo di esso toccava ad un altro e cosi avanti. Siccome v'ha molta rivalità fra i maschi, è probabile che le femmine non solo li scoprissero pei suoni che emettevano, ma che, come le femmine degli uccelli, fossero eccitate o lusingate dal maschio dotato di una voce più simpatica ».

Molto sviluppati sono pure gli apparati fonanti negli Ortotteri soprattutto in quelli appartenenti alle tre famiglie seguenti: Achetidae, Locustidae e Acrididae. Anche negli Ortotteri è il maschio che ha l'apparato del suono più sviluppato.

Nei Coleotteri per lo più ambedue i sessi possono produrre certe maniere di suoni, e in questo caso i suoni emessi servono come di mutuo richiamo.

Emettono suono fra i Coleotteri, fregando le elitre e l'addome, l'Oxycheila tristis Fab., e varie specie di Cychrus; fregando la base delle elitre sul primo segmento addominale, le specie del genere Necrophorus; fregando il pigidio contro i] margine delle elitre, l'Hydrobia Hermanni Fab., l'Hydrophilus piceus (probabilmente il suono emesso da questa specie è prodotto in questo modo), molte specie di Copris e di Trox 
fra i Lamellicorni, e di Acalles fra i Curculionidi. Moltissimi Longicorni poi producono una sorta di stridulazione fregando il protorace sul mesotorace. Accennerò in ultimo all'Anobium striatum, il quale percuote colla testa l'interno della galleria che si è scavata nel legno, producendo una serie di colpi secchi che servono di richiamo sessuale (1).

Colla scelta sessuale e coi caratteri sessuali secondari viene a legarsi strettamente una serie di fenomeni importantissimi, chiamati fenomeni di polimorfismo.

I fenomeni dagli Autori compresi colla denominazione di fenomeni di polimorfismo sono molto complessi e sono l'effetto di varie cause variamente operanti. Cosi, ad esempio, abbiamo una sorta di polimorfismo dipendente dal variare dell'età, una sorta di polimorfismo negli animali sociali dipendente dai vari uffici a cui l'animale deve attendere, una sorta di polimorfismo dipendente dalle stagioni (2), dall'adattamento, dal mimismo, ecc., e finalmente una sorta di polimorfismo prodotto dalla scelta sessuale. Io non mi occupo ora che di quest'ultima maniera di polimorfismo.

Ecco come il Wallace (3) definisce il polimorfismo: "Con questo nome io indico la coesistenza nella stessa località di due 0 più forme distinte che non sono collegate da forme inter-

(1) L'Acherontia atropos fra i Lepidotteri produce pure un rumore aspro sull'origine del quale non si hanno ancora cognizioni ben sicure.

(2) Citerò qualche esempio di questa maniera di polimorfismo che non è infrequente negli insetti, soprattutto nei Lepidotteri:

L'Anthocharis belemia Esp. ha in primavera delle belle macchie argentate sulle ali, mentre in estate le stesse macchie non lo sono e costituisce allora la varietà glauce Hubn.

L'Anthocharis belia Cr. Esp. presenta lo stesso fenomeno e la forma estiva a macchie non argentate costituisce la varietà ausonia Hubn.

La Vanessa levana Linn. ha il fondo delle ali di color fulvo in primavera, di color nero invece in estate (var. prorsa Linn.).

La Metrocampa margaritaria Linn. è in primavera molto più grossa che non in estate.

Le crisalidi dei Papitio Machaon Linn., podativius Linn., sono ordinariamente verdognole in estate, grigie invece in inverno.

Nei Coleotteri la Cassida murraea Linn. è verde in primavera ed ordinariamente rossa in estate.

(3) Op. citata. 
medie, e ciò nondimeno sono spesso prodotte da progenitori comuni. Queste forme distinte non si presentano, in generale, che nel sesso femminile, e i loro discendenti invece di essere forme ibride, forme cioè che presentano dei punti di rassomiglianza coi due progenitori, sembrano riprodurre in proporzioni variabili ciascuna delle forme distinte ».

Relativamente molti oramai sono i fatti di polimorfismo stati osservati negli insetti, e molto probabilmente « un esame diligente farà riconoscere il polimorfismo in molti casi che si suppongono essere semplici varietà ».

I Lepidotteri offrono, soprattutto quelli appartenenti al genere Papilio, molti casi di polimorfismo, e a questo proposito si consulti l'opera già ripetutamente citata del Wallace.

Casi di polimorfismo incontransi pure nei Rincoti e nei Coleotteri. Io mi limiterò a menzionare due casi di polimorfismo in insetti di questo ultimo ordine.

Il primo caso si osserva nelle femmine dell'Hydrophilus piceus Linn (1).

Le elitre, che son quelle che nelle femmine dell'Hydrophilus piceus Linn. presentano appunto il fenomeno del polimorfismo, mostrano, oltre alle altre particolarità, lungo il loro margine esterno una depressione in forma di solco, che cominciando agli angoli anteriori di esse, si prolunga verso il loro apice gradatamente diminuendo: nel maschio e nella prima forma di femmina, in quella cioè da lungo tempo conosciuta, queste depressioni si possono, per rispetto alle altre forme, dire liscie, non presentando esse che qualche striatura longitudinale, qualche linea di punti impressi, e qualche rugositå più o meno spiccata.

Le femmine appartenenti alle altre forme presentano tutte, quali piú quali meno sviluppato, un rialzo careniforme nella prima metà della solcatura anzidetta.

Questo rialzo in forma di carena, negli individui in cui si trova più evidente, si presenta come nella Fig. 13, e prende origine ad una distanza dalla base dell'elitra eguale a circa un

(1) I. Camerano: " Polimorfismo nella femmina dell'Hydrophitus piceus Linn. . Atti della Reale Accad. delle Scienze di Torino, vol. XII, 1877. 
settimo della lunghezza dell'elitra stessa: dapprima poco cospicuo, cresce poi a mano a mano nel discendere in basso, raggiunge un massimo di sviluppo, e gradatamente torna a diminuire per iscomparire al tutto ad una distanza eguale a poco più di tre settimi della lunghezza dell'elitra misurata a partire dalla base.

Negli individui in cui non è molto sviluppato, quantunque prenda origine presso a poco sempre alla stessa altezza, non giunge tuttavia cosi in basso. Il punto in cui comincia il rialzo dista dalla base dell'elitra di una quantità quasi costante, cioè di quattro o cinque millimetri; la lunghezza del rialzo è invece variabile: se ne hanno, per non citare che gli estremi, di m. 0,014 e di m. 0,006 .

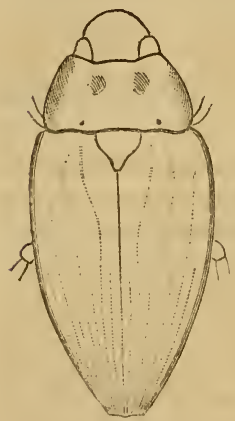

Fig. 12.

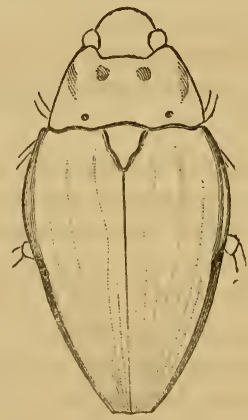

Fig. 13.

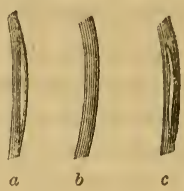

Fig. 14 .

Le femmine poi, che presentano il sopraddetto rialzo careniforme, possiamo ancora dividere in due categorie a seconda del modo in cui l'orlo esterno delle elitre, come si sa alquanto risvoltato in alto, si comporta col rialzo stesso. Nelle une forma col margine esterno del rialzo careniforme un solco bene spiccato (fig. $14 c$ ); nelle altre invece si fonde col rialzo, ed il solco, cosi evidente nella forma precedente, si trova qui ridotto all'ultima porzione del rialzo careniforme (fig. $14 a$ ). In altri termini, nella prima il rialzo si trova intieramente staccato dal margine esterno dell'elitra, nell'ultima, invece, non è libera che la sua estremità inferiore. 
Riassumo ad ogni modo, per maggior chiarezza, le forme descritte nella tavola seguente:

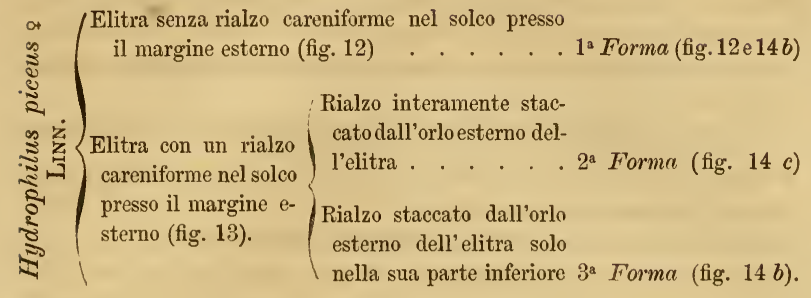

Fra la prima e la seconda forma non sono rari i passaggi: rarissimi invece fra la prima e la terza e fra la seconda e la terza.

Un caso di polimorfismo analogo al precedente ci offrono pure le femmine di varie specie del genere Dytiscus. Si consulti a questo proposito:

A. Preudhomme de Borke. - Notice sur les femelles à élytres lisses du Dytiscus marginalis Linn. Ann. de la Soc. Ent. de Belgique, vol. XII, 1868-69 e XIII, 1869-70.

I fenomeni di polimorfismo sopra accennati sono un argomento buonissimo per la dimostrazione della teoria della scelta sessuale; essi ci rappresentano le gradazioni intermedie dello sviluppo delle differenze fra i sessi, dei caratteri sessuali socondari, gradazioni che si sono accidentalmente conservate e che molto probabilmente tendono a scomparire.

\section{Bibliografia.}

DaRWIN. - Op. citata.

WALLACE. - Op. citata.

Canestrini. - Op. citata.

H. A. Pagenstecher. - Allgemeine Zoologie. Erster Theil, 1875. 
D. A. Weismann. - Ueber den Saison-Dimorphismus der Schmetterlinge. Annali del Museo Civico di Storia naturale di Genova, vol. VI, 1874, pag. 209, tav. VIII, IX.

Revtrer. - Remarques sur le polymorphisme des Hémiptères. Amn. Soc. Ent. Franc. 1875.

KraAtz. - Ueber Pterygo-Dimorphismus bei Caraben. Deutsche Ent. Zeitsch. 1877 , p. 64 , tav. I, $N^{\circ} \Pi$, fig. 1.

L. Camerano. - Note intorno ai caratteri sessuali secondari di alcuni coleotteri. Atti R. Accad. Scienze di Torino, vol. XIII, 1878.

V. Ghilianı. - Ermafroditismo e dimorfismo riuniti in un medesimo lepidottero. Boll. Entomol. Ital., anno IX, 1877.

De GEER. - Mémoire pour servir a l'histoire des Insectes, t. III.

Gadreav. - Essai sur la stridulation des Insectes. Ann. de la Soc. Ent., 1837.

Yersin. - Sur la stridulation des Ortoptères. Bull. de la Soc. Vaudoise des sc. nat., vol. III, 1847.

S. SCUDDER. - Notes on the stridulation of some New-England Orthoptera of the Boston Nat. hist. Soc., vol. XI, 1868.

Lannors. - Die Ton und Stimmapparate der Insecten in anatomisch-physiologischer Bezeihung. Zeitschr. für wissensch. Zool., vol. XVII, 1867.

- Ueber ein den sogennanten Tonapparat der Cikaden, ecc. Zeitschr für wissensch. Zool., 1872, t. XXII.

Westring. - Underretning om det of ham opdagede Stridulationsorgan hos en Arachnide. Kroyer's Nataurhostorik Tidskrift, 1842, vol. IV. - On Insekternes Stridulations organe. Op. eitata, $2^{a}$ ses., vol. II, 1847.

Solier. - Observations sur quelques particularités de la Stridulation des Insectes. Ann. Soc. Ent., 1837, vol. VI.

Latreille. - De l'organe musical des Criquets et dés Truxales. Ann. du Muséum d'hist. nat., vol. VIII.

Gruber. - Anhang zu der Abhandlungen über die Tonapparate der Locustiden. Zeitschr. für wissensch. Zool., 1872, vol. XXII.

Passerin1. - Osservazioni sopra la Sphinx atropos. Pisa, 1828.

Carus. - Ueber die Stimmwerkzeuge der italianischen Cicaden. Ann. Zur. Naturw., 1829.

Carlet. - Mémoire sur l'appareil musical de la Cigale. Ann. des scienc. nat., 1877 , ses. 6 , vol. V.

C. Lepori. - Nuove ricerche sopra l'organo sonoro delle Cicale. Boll. Soc.Ent. Ital. Anno I, 1869, p. 221. 


\section{INTEGUNENTO DEGLI INSETTI}

Il Malpighi, lo Swammerdam, il Leuwenhoek, il Lyonnet, lo Straus-Durcheim, l'Audouin, il Savigny, ecc., furono i primi, come io ebbi già l'occasione di dire, a far vedere la complicatezza della struttura degli insetti. Oggigiorno il numero dei lavori che si posseggono intorno all'anatomia degli insetti è molto grande. Le nostre conoscenze tuttavia intorno alla loro struttura non sono ancora molto sicure, e molti fatti devono ancora venire accertati.

Cominciamo il nostro studio dall'esterno. Cominciamo cioè da quel complesso di parti dure che, siccome tutti sanno, costituiscono l'involucro esterno degli insetti e che è chiamato dermascheletro.

L'esame della struttura intima della pelle degli insetti ci fa vedere che essa non è foggiata come la pelle dei vertebrati, con un derma connettivo, e con un epiderma cellulare, ma che essa consta invece di uno strato esterno chitinizzato (strato, dirò di passaggio, che molti autori distinguono col nome di epiderma) e di una membrana molle non chitinizzata (corrispondente al chorion di molti autori).

Un altro carattere che distingue pure la pelle degli insetti da quella della maggior parte degli animali superiori si è la mancanza in essa di fibre muscolari destinate ai movimenti della pelle stessa.

Inoltre vi ha un grande numero di canaletti (pori canali) che attraversano perpendicolarmente lo strato chitinoso, ora essendo di diametro finissimo, ora invece alquanto grosso, ora conservando per tutta la loro lunghezza un diametro eguale, 
ora invece allargandosi verso la loro parte esterna, talora in fine ramificandosi fra loro.

Molto vario è il contenuto dei pori canali. Essi possono contenere sostanze liquide di varia natura, oppure essere semplicemente ripieni d'aria, come avviene appunto in alcune specie del genere Hydrometra ed in altre specie appartenenti allo stesso gruppo di Rincoti. Questo fatto probabilmente è in rapporto colla loro vita acquatica.

Nei pori canali più grossi, infine, si possono trovare anche dei prolungamenti papillari molto sottili che partono dallo strato molle sottostante.

I pori canali dello strato chitinizzato vengono dal Leydig (1) considerati come omologi dei corpuscoli del tessuto connettivo.

Riassumendo, le parti essenziali che caratterizzano la pelle degli insetti sono:

$1^{\circ}$ Lo strato esterno chitinizzato;

$2^{\circ}$ Lo strato interno molle;

$3^{\circ}$ I pori canali.

\section{Strato ESTERno ChITINizZato.}

La superficie libera dello strato esterno ci si presenta molto variamente foggiata e molte volte ha certi disegni e certe linee disposte in modo da offrirci l'aspetto di un tessuto cellulare.

Questo fatto ci farebbe supporre facilmente la presenza di un sottilissimo strato cellulare epidermico, composto come negli animali superiori di cellule epiteliali pavimentose. Non pare tuttavia che questo strato esista e che si tratti di vere cellule.

I disegni variano assai ed ora vi hanno delle striscie finissime, ora dei tubercoletti, ora dei rialzamenti più o meno spiccati (ora lisci, ora seghettati), ora delle rughe e delle grinze, o intralciantisi confusamente fra loro, o disposte a mo' di stelle, ecc. (2).

Lo strato chitinizzato, esaminato nel suo complesso, appare

(1) «Lehrbuch der Histologie des Menschen und der Thiere».

(2) Si consulti a questo proposito la memoria del Cornalia intitolata: «Sopra i caratteri microscopici offerti dalle cantaridi e da altri coleotteri facili a confondersi con esse ". Memorie della Società italiana di Scienze naturali, vol. I, Milano 1865. 
formato da un grande numero di lamelle regolarmente sovrapposte o stratificate. Nei primi stadi dello sviluppo degli insetti è molle e non acquista che a poco a poco, e non in tutti i suoi punti in egual grado, la durezza della sostanza cornea, od anche, come avviene in qualche rarissimo caso per deposizione di sali calcari, la durezza dell'osso.

Raramente nello integumento degli insetti si osserva il fatto della presenza di sali calcari (cosa invece frequentissima in altri artropodi : miriapodi e crostacei). Il Leydig (1) ha trovato corpuscoli calcarei nella pelle delía larva dello Stratiomys chamaeleon.

Il modo di accrescimento dello strato chitinizzato è analogo a quello dell'epidermide degli animali vertebrati, si fa cioè dall'interno all'esterno e il tessuto si rinnovella di tratto in tratto collo staccarsi degli strati più vecchi e più esterni e col sostituirsi ad essi i più interni e i più giovani.

Questo rinnovellarsi dello strato più esterno della pelle costituisce negli insetti quel fenomeno che volgarmente è detto muta o cambiamento della pelle.

Questo fatto è molto importante per la vita degli insetti e merita che noi vi spendiamo intorno qualche parola.

Gli insetti mutano varie volte la pelle durante la loro vita larvale; raramente ciò fanno quando hanno compiuto il loro sviluppo e sono pervenuti allo stato di insetto perfetto. Le effimere, ad esempio, cambiano la pelle una volta ancora dopo che hanno compiuto la loro metamorfosi in insetto perfetto.

Ordinariamente l'insetto poco tempo prima del cambiamento della pelle cessa di mangiare, dal muoversi qua e là e cade in uno stato analogo a quello del letargo.

In questo tempo si osserva in generale la formazione di un leggiero essudato sieroso fra il vecchio strato epidermico e lo strato mucoso sottostante. Passato questo periodo, che chiameremo di riposo o di preparazione, ne succede un altro di lavoro in cui l'insetto si mostra molto agitato, irrequieto, si muove qua e là e cerca con ogni sforzo di svestire l'involucro esterno oramai divenutogli inutile.

(1) «Ueber Kalkablagerung in der Haut der Insecten ». Archiv. fur Naturgeschichte, 1860, I, p. 156, tav. VII, fig. 1, 2, 3. 
Nella maggior parte dei casi l'involucro si rompe nella regione dorsale e l'insetto libera prima il capo, poi il corpo e per ultimo le zampe.

Spesso, insieme collo strato esterno della pelle si stacca pure (come già aveva osservato lo Swammerdam) (1), la tunica interna od epiteliale che riveste internamente l'ultima parte del canal intestinale ed i grossi tronchi tracheali.

Appena uscito dall'involucro l'insetto ha colori sbiaditi. In qualche caso anzi è al tutto scolorito, e cerca per lo più di sottrarsi all'azione immediata della luce, spesso non senza avere, non si sa per qual ragione, mangiata molto avidamente la propria spoglia. Questo fatto non si verifica solamente negli insetti. Gli anfibi e molte lucertole che, come gli insetti, mutano varie volte la pelle, divorano pure avidamente la pelle stessa appena questa si è staccata dal loro corpo.

Il colore sbiadito e qualche volta al tutto mancante dello involucro esterno degli insetti che si osserva immediatamente dopo che essi hanno compiuto la loro metamorfosi, ha spesso fatto credere a casi di albinismo. Avviene non raramente nella comune blatta, ad esempio, di trovare alcuni individui, fra i molti che infestano le nostre case, che sono di color bianco. Questi individui sono quelli che hanno da poco tempo cambiato la pelle. Dopo ventiquattro ore al più il loro involucro esterno prende il colore bruno caratteristico della specie.

Un fatto analogo si osserva pure in molti Coleotteri carabici, ad esempio nei generi Harpalus (H. ruficornis Fal., H. griseus Panz., H. aeneus Fab.), Stomis, Broschus, ecc.

Il numero delle mute $o$ dei cambiamenti della pelle varia negli insetti a seconda non solo degli ordini, ma anche delle famiglie e dei generi. Ordinariamente vi hanno tre mute di pelle prima della metamorfosi in insetto perfetto. In qualche caso se ne osservano anche dieci come nella larva del Bombyx caja (2).

Gli antichi, dirò in ultimo, non avevano idee giuste intorno al fenomeno della muta della pelle negli insetti. Lo Swam-

(1) «Biblia Naturae», p. 129, 134, 239, ecc.

(2) Kirby e Spence: *Introduction to Entomology » t. III, p. 196, 1826. 
merdam, ad esempio, credeva che gli insetti nascessero rivestiti di una serie di involucri sovrapposti e che di essi si spogliassero a mano mano che crescevano.

Le ricerche dei moderni hanno invece posto fuori di dubbio che il nuovo rivestimento cutaneo, quello che rimane dopo la caduta del vecchio, non preesiste, ma si forma al tempo della muta e compie il suo sviluppo dopo la muta.

L'involucro esterno degli insetti, che nei primi stadi del loro sviluppo è molle e flessibile, si indurisce poco a poco col crescere dell'insetto e prende l'aspetto e la consistenza della sostanza cornea; in una parola, si chitinizza.

Il tessuto chitinoso, che ha l'aspetto in generale del tessuto corneo, è tuttavia da questo totalmente diverso nella sostanza, nella sua composizione chimica e si distingue facilmente per la sua proprietà di essere inattaccabile dagli alcali.

Esso anzi resiste all'azione di una soluzione concentrata e bollente di potassa e di soda, ed inoltre non dà, bruciandolo, che leggerissimi vapori ammoniacali. Anche quest'ultima proprietà serve a farlo agevolmente distinguere dalla sostanza cornea.

La chitina venne per molto tempo considerata come un principio immediato. Il Peligot (l) ha dimostrato invece che essa risulta composta da cellulosa e da una sostanza albuminoide.

La formola più recente della chitina è la seguente:

$$
\mathrm{C}^{9} \mathrm{H}^{15} \mathrm{NO}^{6} \text {. }
$$

Strato MOLLE NON ChitinizZato.

Lo strato molle non chitinizzato che si trova al disotto dello strato chitinizzato, è costituito in massima parte da sostanza connettiva che può assumere varii aspetti; ora presenta cellule ben evidenti, ora invece la struttura cellulare scompare e non si hanno più che nuclei trasparenti involti da una sostanza finamente granulosa.

Dei pori canali ho già parlato.

Ritornando allo strato esterno della pelle degli insetti, noi

(1) "Sur la composition de la peau des vers à soie ». Compt. Rend. de ''Acad. des Scienc., 1858, t. XVII. 
osserviamo sopra di essa un grande numero di escrescenze, di produzioni speciali variabilissime di forma e di ufficio. Queste produzioni (peli, squame, ecc.) sono costituite essenzialmente di tessuto chitinoso, e sorgono per lo più sopra i fori cutanei più cospicui. Essi sono così in comunicazione coi più grossi pori canali. Queste produzioni chitinose si possono considerare come semplici prolungamenti della pelle, esse sono foggiate a no' di tubo a fondo cieco o allungato come un pelo ordinario - dilatato in modo da costituire una vescicola peduncolata la quale appiattendosi prende la forma di una fogliolina o di una squama o scaglietta.

Si possono quindi dividere le escrescenze della parte esterna dell'involucro chitinoso degli insetti, tenendo conto della forma che esse presentano, in due grandi categorie:

1. Peli.

2. Squame.

Ripeto tuttavia che le scaglie ed i peli hanno la stessa origine e sono in fondo la stessa cosa.

Le forme dei peli sono molte. Io mi limiterò a dare un'idea delle principali.

Si ha anzitutto la peluria che riveste in molti casi varie parti dell'insetto, soprattutto le parti inferiori., I peli che la costituiscono sono finissimi e corti. Poi vengono i peli propriamente detti, i quali si trovano molto frequentemente sul corpo di molti insetti ed abbondano soprattutto sul corpo di molte larve di lepidotteri. La lunghezza dei peli propriamente detti puó variare da un millimetro o poco più ad un centimetro o ad un centimetro e mezzo.

In qualche caso, come ho già detto, i peli possono essere urticanti.

I peli possono essere o lisci, come nella maggior parte dei casi, 0 avere invece un grande numero di spine laterali, come, ad esempio, in qualche specie di imenotteri del genere Bombus.

In questo ultimo caso questi peli sono simili a quelli del corpo di alcuni chirotteri.

Dai peli propriamente detti si può passare molto facilmente alle setole ed alle spine, le quali in fondo non sono altro che peli rigidi od ingrossati. Le setole e le spine si incontrano per 
lo più esclusivamente nella parte inferiore dell'addome e sulle estremità.

Gli insetti acquaiuoli, soprattutto ditiscidi ed emitteri, presentano una forma speciale di peli, i quali, collocati ai lati delle zampe posteriori e mediane, trasformano le zampe stesse in remi. I peli in questo caso sono per lo più appiattiti ed hanno l'aspettò di laminette (Figure 15 e 16).

Altre forme di peli troviamo ancora senza allontanarci dalle zampe. Gli articoli dei tarsi in moltissimi insetti sono rivestiti inferiormente da numerosi e fini peli, i quali hanno forme che variano nelle varie famiglie e concedono all'animale di camminare sopra superficie lisce verticali. Questi ${ }^{\text {Tarso deil' }{ }_{\text {philus }} \text { piceus. }}$ peli funzionano in certi casi come ventose.

Un'altra categoria di peli in cui la forma e l'uffizio di ventosa è più spiccato si trovano pure rivestire gli articoli dei tarsi delle zampe anteriori per lo più molto sviluppati trasversalmente, raramente anche le mediane dei maschi di molti coleotteri. Questi peli che formano delle spazzolette molto fitte servono al maschio per tener ferma la femmina durante l'accoppiamento.

Variano questi peli moltissimo nella loro forma. Ora sono piccolissimi e la loro grossezza non supera qualche decimo di millimetri (Cicindela campestris, C. hybrida, ecc.). Ora invece possono avere la grossezza di circa mezzo millimetro, come si vede in vari lamellicorni.

Le figure qui unite rappresentano qualcuna delle forme più spiccate e caratteristiche di questi peli (Fig. 17).

Peli di tal fatta non si incontrano del resto solo nelle parti inferiori dei tarsi dei maschi, ma in alcuni coleotteri il mento dei maschi possiede

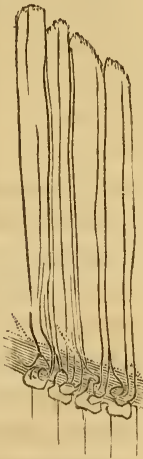

Fig. 16. Gyrinus natator (da Targioni Tozzetti). pure una fitta spazzolina di questi peli ventose, i quali compiono lo stesso uffizio di quelli dei tarsi; sono in questo caso molte fra le specie del genere Geniates.

La presenza dei peli ventose, che costituiscono uno dei più importanti caratteri sessuali secondari, il loro grande sviluppo 
nei soli maschi, si può facilmente spiegare colla legge della scelta sessuale.

Abbiamo finalmente un'ultima categoria di peli che sono sparsi sopra varie parti del corpo di certi insetti e che si possono chiamare peli tattili. Di questi parlerò più a lungo trattando del senso del tatto.

Diro in ultimo che i peli, oltre che nella forma, variano assai anche nel colore e possono essere o di un bianco argenteo, ed allora essi sono internamente ripieni d'aria, cio che si osserva in molti coleotteri soprattutto lamellicorni, ad esempio nei generi Cetonia, Polyphylla, Melolontha, ecc.; ovvero variamente colorati, ed allora la loro colorazione puó dipendere dalla presenza di un pigmento speciale o da speciali strutture della loro superficie. In quest'ultimo caso la loro colorazione è prodotta da fenomeni di interferenza luminosa.

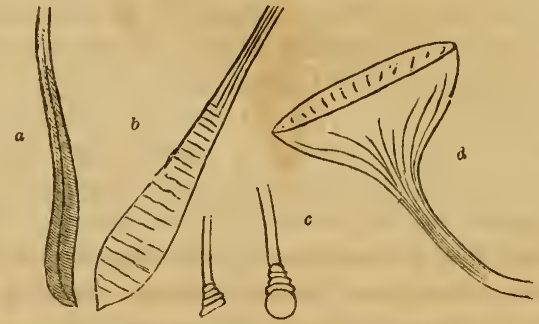

Fig. 17. - $a-$ Cicindela campestris. $-b$-Geniates barbatus. $c-$ Broschus cephalotes. $-d-$ Dolichus favicornis.

SQUAME.

Le squame, come ho già detto, non sono altro che peli molto dilatati ed appiattiti.

I lepidotteri sono fra tutti gli insetti quelli che meglio si prestano allo studio delle squame, in quanto che le loro ali portano un grande numero di queste produzioni epidermiche. Ad esse anzi devono i lepidotteri le splendidi colorazioni delle loro ali. Le squame non sono tuttavia esclusive alle ali dei lepidotteri, se ne osservano anche sulle ali di qualche neurottero (Friganea) e sulle elitre e sul corpo di molti coleotteri (generi Hoplia, Eutimus, Cyphon, ecc.), ed anche in qualche 
genere di ortotteri (Lepisma, ecc.). Sulle ali dei lepidotteri le squame sono disposte in serie embricate, ed ora ricoprono intieramente l'ala stessa (generi Vanessa, Pieris, Papilio, ecc.), ora ne lasciano allo scoperto delle porzioni più o meno grandi (generi Sesia, Hymenitis) (Fig. 18).

La forma delle squame varia non solo da specie a specie, ma anche nella stessa specie secondo la porzione dell'ala in cui esse si trovano impiantate. In generale le squame sono molto larghe nel mezzo dell'ala, si allungano invece e tendono ad assumere la forma di peli andando dal mezzo dell'ala verso il corpo dell'insetto e verso la periferia dell'ala stessa.

La figura 19 dà un'idea delle principali forme di queste produzioni epidermiche meglio di qualunque descrizione.

Le squame che ricoprono le ali dei lepidotteri appaiono co-

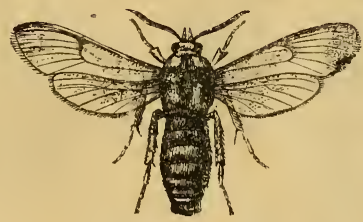

Fig. 18. Sesia apiformis.

stituite, come dice il Deschamps (1), da due e spesso anche da tre membrane sovrapposte. Questa struttura, dirò di passaggio, si può scorgere bene nelle scaglie che vennero rotte per qualche accidente.

Il Deschamps dice pure che i granuli da cui risulta la colorazione della squama si trovano sempre sulla membrana superiore, e che le strie quando esistono si trovano sempre sulla seconda lamina.

Le strie delle squame sono esse pure variabilissime nella forma; per lo più vi ha un grande numero di strie longitudinali finissime, le quali ora sono lisce, ora cordoniformi attraversate da altre, ora contengono fra loro dei tubercoli.

(1) Bernard Deschamps "Recherches microscopiques sur l'organisation des ailes des Lépidoptères s, Annal. des Sc. Nat., 2a Ser., vol. III, p. 113, tavola $3^{\mathrm{a}}$ e $4^{\mathrm{a}}$. 
Le strie finissime che solcano le squame sotto l'azione della luce dànno origine ad elegantissimi fenomeni di interferenza, i quali, combinati coi colori dello strato pigmentale delle squame stesse, dànno a queste tutte quelle colorazioni eleganti e svariate che noi ammiriamo sulle ali delle farfalle.

Intorno poi, dirò in ultimo, alla natura delle strie delle squame, alla loro origine, al loro uffizio, si sono fatte varie ipotesi. Il signor B. Deschamps (1), ad esempio, pone innanzi l'ipotesi che queste strie non siano altro che le ultime diramazioni dei rami tracheali che scorrono numerosissimi inferiormente fra le membrane alari. Questa ipotesi verrebbe a
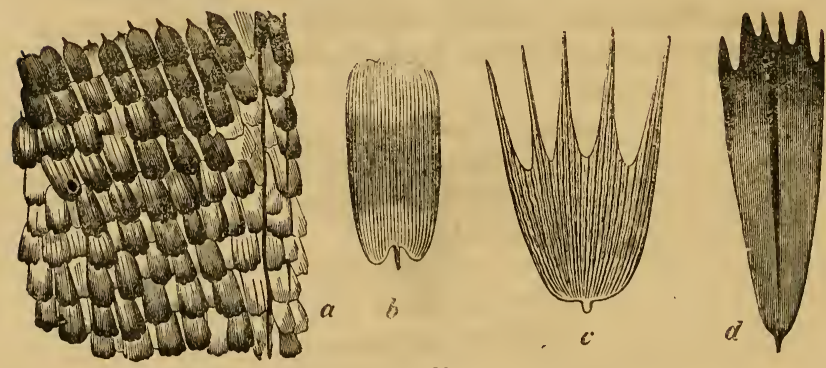

Fig. 19.

$a-b$ - Vanessa Io. $-c$-Acherontia Atrops. $-d$-Catocala elongata.

concordare con quella che considera le ali degli insetti come organi di respirazione esterni, come stimme ipertrofiche (2).

Oltre ai peli ed alle squame noi dobbiamo tener conto ancora di una sorta di polvere biancastra finissima che spesso ricopre a guisa di un bianco velo l'integumento esterno degli insetti.

Questa polvere biancastra è formata esclusivamente da granuli finissimi di cera che viene alla superficie esterna della pelle passando attraverso ai canaletti che, come ho già detto, solcano in gran numero lo strato chitinizzato.

(1) Op. cit., p. 129.

(2) F. Plateau «Qu'est-ce que l'aile d'un Insecte? * Stettiner entomologische Zeitung, XXXII, pp. 33-42, tav. 1, 1872. 
Il signor F. Dujardin (1) ha fatto uno studio diligente di questo fenomeno ed ha trovato che è molto grande il numero degli insetti che producono la cera. Noterò di passaggio che la cera prodotta dagli insetti è analoga a quella che si trova ricoprire la superficie esterna di molti frutti.

Fra gli insetti produttori di cera vengono in prima linea l'ape e vari altri imenotteri. Agli imenotteri tengon dietro immediatamente i rincoti, soprattutto le specie appartenenti alla famiglia delle cicale. In qualche specie di cicale (ad esempio, nella Lystra pulverulenta. Curier, Règne Animal. Insectes, tav. 97, fig. 2) la cera ci si presenta sotto la forma di filamenti relativamente molto lunghi che partono dagli ultimi segmenti dell'addome e dànno all'animale un aspetto strano.

Sono pure da annoverarsi fra le specie di insetti produttori di cera le seguenti:

Myxzoxylus mali,

Coccus o Lecanium vitis,

Aleurodes chelidonii,

Jasus prasinus,

Notonecta glauca (2),

Gerris lacustris (2),

Libellula depressa,

\section{» coerulescens.}

Dando ora un'occhiata complessiva alle colorazioni dell'integumento degli insetti, noi vediamo che esse possono provenire o dalla presenza di un pigmento speciale o da speciali strutture della superficie esterna, le quali dànno luogo a fenomeni di interferenza.

Il pigmento può trovarsi cosi uniformemente diffuso da non lasciare scorgere coi mezzi attuali di osservazione alcuna struttura speciale, ovvero può avere una struttura granulosa.

Il pigmento (3) inoltre può trovarsi o nello strato chitiniz-

(1) « Mémoire sur l'étude microscopique de la cire, appliquée à la recherche de cette substance chez les animaux et les végétaux ». Annal. des Sc. Nat., $3^{\text {a }}$ Ser., vol. XII, p. 250, 1849.

(2) In queste specie il fenomeno della produzione della cera è molto probabilmente in rapporto colla loro vita acquatica.

(3) Nell'involucro esterno degli insetti soprattutto nelle elitre di certi co- 
zato, o nello strato molle, o infine in tutti e due gli strati. Nella pelle degli insetti si trova un numero più o meno grànde di ghiandole, le quali o sono monocellulari come quelle di molti coleotteri, ovvero sono policellulari come le ghiandole odorifere delle specie dei generi Pentatoma, Acanthia, ecc., fra i rincoti, e Aromia, fra i coleotteri (1).

Talvolta le ghiandole cutanee sono in stretto rapporto coi peli, come si osserva nella pelle delle larve di molti lepidotteri del genere Bombyx. In questo caso questa sorta di ghiandole preparano un umore irritante, umore in cui è stato trovato una quantitá più o meno grande di acido formico. Di qui la ragione per cui i peli di molti bruchi (Bombyx pytiocampa, B. processionea, B. quercus, Liparis auriflua, Lithosia caniola) (2), quando vengono toccati, irritano la pelle dell'uomo e forse anche degli altri animali a integumento molle.

Il Leydig, dirò in ultimo, considera come appartenenti alla categoria delle ghiandole cutanee vari organi speciali di secrezione, come le ghiandole velenifere di molti imenotteri e le ghiandole anali di molti coleotteri: Dytiscus, Brachinus, ecc.

leotteri esiste un olio colorato analogo forse a quello che colora i peli dei mammiferi. Questo olio è bruno ad esempio nel genere Melolontha, rosso invece nel genere Crioceris. Si consulti a questo proposito Bernard Deschamps \& Recherches micoscropiques sur l'organisation des élytres des coléoptères ». Annal. $d$. Sc. Nat., 3a Ser., vol. III, p. 354, 1845.

(1) Per maggiori particolari intorno alle ghiandole cutanee degli insetti, vedi Leydig, Op. cit.

(2) Si consulti a questo riguardo Réadmur, Mémoires, ecc., t. II, p. 179. Padl Gervais et Van Beneden, Zoologie Medicale, Paris, 1859, vol. I, p. 353. 


\section{Bibliografia.}

KirbY et SpExce. - Introduction to Entomology, 1826.

Gegenbaur. - Op. cit.

L. Doforr. - Études anat. et physiol. sur une Mouche. Mém. de l'Acad. des Sc. Sav. étrang., 1845, vol. IX.

A. Opier. - Mém. sur la composition chimique des parties cornées des Insectes. Mém. de la Soc. d'Hist. nat. de Paris, 1823, vol. I.

LASSAIGNE. - Sur le téssu tégumentaire des Insectes. Comptes rendus de $\boldsymbol{l}^{t} A c$. des sciences, 1843, vol. XVI.

Paren. - Propriété distinctives entre les membranes végétales et les enveloppes des Insectes et des Crustacés. Comp. rend., 1843, vol. XVII.

Peligot. - Sur la composition de la peau des Vers à Soie. Comp. rend., 1858. vol. XVII.

Strauss DürckнEim. - Considérations sur l'anatomie comparée des animaux articulés, ecc., 1828.

Burmeister. - Handbuch der Entomologie, 1833, vol. I.

Castelnat. - Histoire naturelle des Insectes coléoptères, vol. I, 1840. Parigi.

N. B. Bross. - Klassen und Ordnungen des Thier Reichs, Artropoda, 1866.

H. Nicolet. - Recherches pour servir à l'histoire des Podurelles. Nouv. Mém. de la Soc. Helvet. des Sc. Nat., vol. VI, 1842, tav. 2.

L. Cameraxo. - Osservazioni intorno alla struttura delle elitre di alcuni coleotteri. Rendiconti delle adunanze della Società Entomologica Italiana, p. $13,1878$. 


\section{VII.}

\section{DERIIASCHELETR0}

Il dermascheletro degli insetti, come ho già detto, si può considerare diviso in tre parti principali che risultano formate da un numero vario di zooniti od anelli.

Il piano di struttura più semplice di un zoonito per ciò che riguarda l'involucro esterno è questo: il zoonito è essenzialmente composto da un cerchio chitinoso diviso in due parti od archi, uno dorsale e l'altro ventrale, fra i quali può venire a frapporsi, o un'appendice o uno stimma. L'arco dorsale è formato da due parti simmetriche che si riuniscono insieme secondo una linea longitudinale e costituiscono il tergum o notum. L'arco ventrale è formato da due parti che costituiscono lo sternum, e di due altri pezzi collocati lateralmente allo sternum che sono gli episterna e gli epimera.

La prima parte del dermascheletro o capo è costituita da cinque zooniti e porta un paio di antenne e le appendici dell'apparato masticatore. Il torace fatto sempre di tre anelli: protorace, mesotorace, metatorace, presenta nella parte dorsale un pronotum, un mesonotum ed un metanotum, e nella parte ventrale un prosternum, un mesosternum ed un metasternum, i quali portano tutti inferiormente, dipendenti dall'arco ventrale, due appendici articolate o zampe. I due ultimi segmenti poi portano pure superiormente due appendici od ali dipendenti dall'arco dorsale. L'addome conta al massimo undici segmenti, i quali, meno in qualche raro caso (Japyx, Campodea), sono sempre privi di appendici articolate (Fig. 20).

Gli ultimi segmenti dell'addome si modificano spesso a costituire gli organi copulatori esterni. 
Nella massima parte degli Ortotteri e dei Nevrotteri il numero dei segmenti dell'addome è di 11. Nei Rincoti è di 10 (Genere Acanthia). Nei Coleotteri è di 9. Nei Lepidotteri e negli Imenotteri di 8.

Dal modo di variare del numero dei segmenti dell'addome nei vari ordini, vediamo come la divisione del lavoro fisiologico si vada facendo sempre più grande risalendo dagli Ortotteri agli Imenotteri. Ciò viene a confermare l'opinione adottata dall'Haeckel e dal Gegenbaur, che cioè gli Ortotteri ed i Nevrotteri siano le forme più antiche; siano gli Archetipi da cui sono provenuti tutti gli altri insetti.

CAPO.

Il capo risulta costituito. essenzialmente da una parte superiore convessa o epicranio, spesso divisa in due da una sutura mediana. La parte anteriore dell'epicranio è detta fronte, la quale è limitata anteriormente dal clipeo, lateralmente dagli occhi, posteriormente dal vertice che costituisce superiormente la parte più sporgente del capo. La parte posteriore del capo viene detta occipite. Le parti laterali anteriori del capo sono distinte col nome di guancie,

e tempia vengono chiamate le parti late- Campodea fragilis vista rali e posteriori del capo stesso.

L'epicranio porta nella sua parte ante-

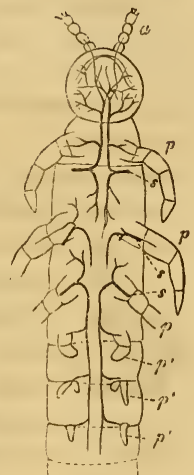
inferiormente $-a$ antenne $p$ piedi $-p^{\prime}$ piedi $r$
dimentali $-s$ stimma.

riore gli occhi e due appendici di forma svariatissima chiamate antenne.

Le antenne sono negli insetti sempre in numero di due, e sono formate da un numero più o meno grande di segmenti od articoli posti l'uno sopra all'altro, e dotati in generale di poca mobilità gli uni rispetto agli altri. Le antenne invece nel loro complesso sono mobilissime e possono a volontá dell'animale volgersi in tutte le direzioni.

La forma delle antenne è molto varia. Ora esse sono foggiate a guisa di un filamento sottile più o meno lungo, ed in questo caso si dicono filiformi; lamelliformi, sono quelle in cui gli ultimi articoli sono foggiati a mo' di lamine (Fig. 21); 
claviformi vengono chiamate quelle antenne in cui gli ultimi articoli sono ingrossati a mo' di clava; penniformi quando per la presenza di un grande numero di barbe laterali hanno l'aspetto di una penna.

Talvolta le antenne sono cortissime, altre volte invece superano due o tre volte la lunghezza del corpo dell'insetto, come si osserva in molte specie di Ortotteri.

In quanto agli usi delle antenne dirò poi trattando degli organi dei sensi.

Al capo, nella massima parte degli animali, tien dietro un collo. Negli insetti esso trovasi in qualche forma appartenente ai Coleotteri, ai Rincoti ed ai Nevrotteri; nella massima parte dei casi tuttavia esso manca.

TORACE.

Molto vario è lo sviluppo dei tre segmenti componenti questa parte del corpo degli insetti e il loro studio è irto di difficoltà.

Il protorace è molto sviluppato nei Coleotteri, negli Ortotteri e nella massima parte dei Rincoti. In questi tre ordini di insetti anzi quella parte che gli Entomologi chiamano corsaletto è costituita intieramente dal primo segmento toracico,

Fig. 21. Melolontha vulgaris. gli altri due segmenti, che sono relativamente poco sviluppati, non sono visibili superiormente salvo il mesotorace, il quale negli insetti appartenenti agli ordini sopra menzionati è visibile all'esterno per una piccola porzione della sua superficie, porzione che piglia il nome di scudetto.

Il protorace è invece poco sviluppato in molti Nevrotteri, negli Imenotteri, nei Lepidotteri e nei Ditteri. Qualche volta anzi è ridotto ad una semplice striscia distinta col nome di collare.

Il mesotorace è molto grande negli Imenotteri, nei Lepidotteri e nei Ditteri, e negli insetti di questi ordini costituisce tutta quella parte che gli Entomologi chiamano corsaletto, parte che come ho detto, è nei Coleotteri, negli Ortotteri, ed in buona parte dei Rincoti formata dal protorace.

Il mesotorace è invece poco sviluppato negli Ortotteri e nei Coleotteri; ha uno sviluppo un po' maggiore in una parte degli Ortotteri e in molti Nevrotteri.

Il metatorace è relativamente grande nei Coleotteri, negli 
Ortotteri ed in vari Nevrotteri, poco sviluppato invece nella maggior parte dei Rincoti, meno ancora negli Imenotteri, nei Lepidotteri e nei Ditteri.

Negli insetti a metamorfosi incompiuta (Ortotteri, Rincoti) le larve e le ninfe presentano i vari segmenti del torace con

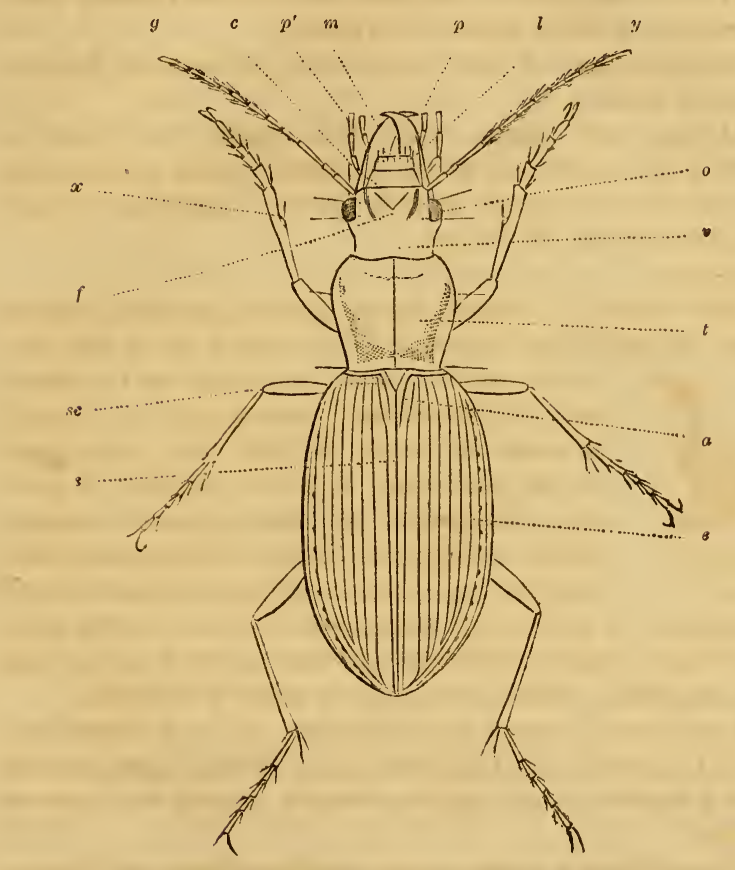

Fig. 22. Sphodrus planus visto superiormente.

$v$-vertex - $f$-frons - $c$ - clypeus - $l$ - labrum - $m$ - mandibulae - o- oculi $p$ - maxillarum lobus exterior - $p^{\prime}$ - palpi maxillares - $y$-antennae - $t$-prothorax - sc-scutellum - e-elytra - s-sutura elytrorum - $x$-lineola abbreviata - $a$ calcar superius tibiarum anticarum.

uno sviluppo analogo a quello che si incontra negli insetti perfetti. Negli insetti a metamorfosi compiuta le cose vanno un po' diversamente. Le larve dei Lepidotteri hanno i segmenti toracici che hanno fra loro un eguale sviluppo. Nelle larve 
dei Coleotteri invece il primo segmento o protorace è notevolmente più sviluppato che non gli altri due.

Il primo segmento toracico ha appendici solamente sull'arco ventrale. L'Acrocinus longimanus fra i Coleotteri longicorni fa tuttavia eccezione a questa regola, inquantochè il suo protorace

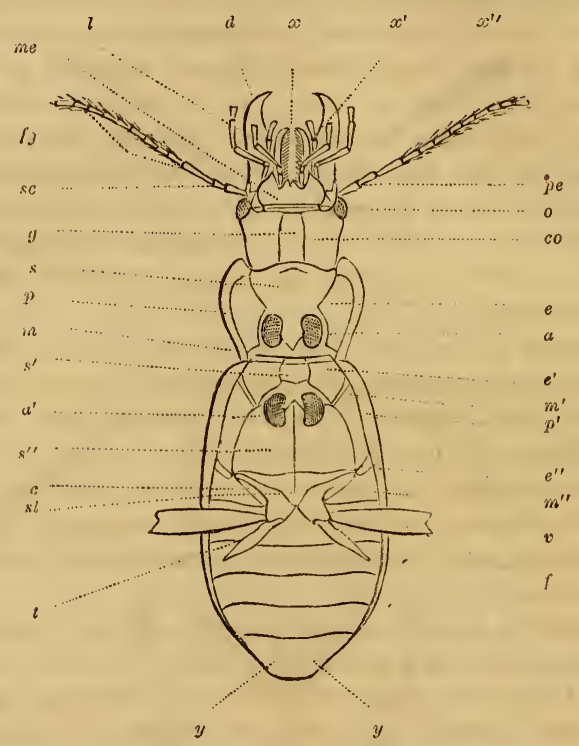

Fig. 23. Sphodrus planus, visto inferiormente.

co-collum - g-gula - o-oculi - me-mentum - sc-scapus antennarum - pe pedicellus antennarum - fg-flagellum antennarum $-d$-mandibulae $-x-m a x i l-$ larum lobus exterior $-x^{\prime}-$ maxillarum lobus interior palpiformis $-x^{\prime \prime}-$ palpi maxillares - $l$-palpi labiales - $p$-epipleura pronoti $-s$ - prosternum - e-episterna prothoracis - $m$-epimera prothoracis - a-acetabula $-s^{\prime}$ - mesoternum - $e^{\prime}$-episterna mesothoracis - $m^{\prime}$ - epimera mesothoracis - $a^{\prime}$ - acetabula intermedia - $p^{\prime}$ - epipleura elytrorum - $s^{\prime \prime}$ - metasternum - sl-metasternellum $-e^{\prime \prime}$ - episterna metathoracis - $m^{\prime \prime}$ - epimera metathoracis - c-coxae posticae - $t$-trochanter - v-segmentum primum ventrale - y-sextum ventrale.

è armato superiormente da un paio di spine mobili che si possono considerare come i rappresentanti di due appendici dorsali.

Gli altri due segmenti toracici hanno appendici e sull'arco dorsale e sull'arco ventrale. Cominciamo il loro studio da queste ultime o dalle zampe. 
Le zampe possono essere di due sorta: possono constare cioè di parti dure articolate fra loro, ovvero essere semplici tubercoli molli subcilindrici o conici sprovvisti di parti dure. Vengono dette zampe vere le prime e false zampe le seconde.

Le zampe vere non sono mai in numero maggiore di tre paia e si trovano sempre ed esclusivamente sopra ai segmenti toracici. Le false zampe possono invece essere in un numero vario di paia, cinque od otto, e per lo più sono collocate sui segmenti addominali. Inoltre non si trovano mai nell'insetto perfetto, ma solo nello stadio larvale. Le false zampe possono poi venire divise in intermedie ed anali a seconda del luogo in cui si trovano, queste ultime sono anche dette piedi spingitori. Le false zampe sono ben spiccate sopratutto nelle larve dei Lepidotteri.

Le zampe vere constano di un numero vario di parti dure articolate fra loro ed hanno il loro punto d'inserzione sull'epimero dell'arco ventrale e sull'orlo laterale dello sterno. In questo punto abbiamo una vera articolazione che ha lo stesso piano di struttura che si osserva nell'articolazione di un condilo nella cavità cotiloidea degli animali superiori.

In qualche caso l'articolazione è assai debole e manca una vera cavità.

Le parti di cui nel massimo numero dei casi è costituita la zampa vera sono le seguenti (Fig. 24) :

$1^{\circ}$ L'anca, la quale per mezzo del trocantino, appendice piccolissima collocata nella sua parte interna, si trova in stretto rapporto coll'epimero;

$2^{\circ}$ Il trocantere, che tien dietro all'anca colla quale è articolato;

$3^{\circ}$ La coscia, che ha forma allungata ed è la parte più robusta della zampa;

$4^{\circ}$ La gamba sottile, allungata e che si presenta molto variamente foggiata;

$5^{\circ}$ Il tarso costituito da parecchi articoli il di cui numero varia da uno a cinque;

$6^{\circ}$ Le unghie od uncini che si trovano impiantate sull'ultimo articolo dei tarsi.

Di tutte le parti or ora enumerate, la gamba ed i tarsi sono 
quelle che più variano nella loro struttura e di queste io ho gia avuto varie volte occasione di parlare.

Le zampe, considerate nel loro complesso, hanno una struttura che è in stretto rapporto col genere di vita dell'insetto.

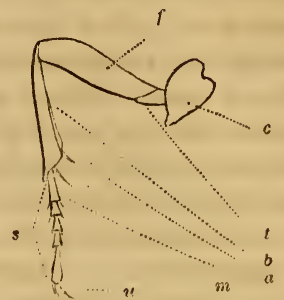

Fig. 24. Zampa di un Carabus.

$c$-coxa - $t$-trochanter - $f$-femur - b-tibia - a-calcaria apicalia $s$-tarsus $-m$-primo articolo del tarso $-u$-unguiculi.

Nei Coleotteri carnivori, ad esempio nei Carabici (Carabus, Cicindela, Megacephala, Tetracha, ecc.), insetti predatori per eccellenza, le zampe sono molto robuste ed allungate. Nei Coleotteri scavatori (Necrophorus) sono molto robuste le zampe posteriori. Nei Coleotteri saltatori (Sagra, Haltica, ecc.); nella Smicra clavipes (Fig. 25) fra gli Imeńotteri le zampe poste-

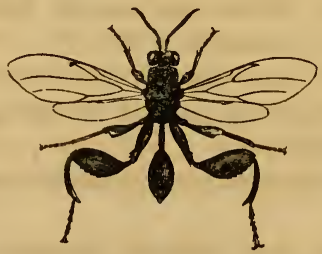

Fig. 25. Smicra clavipes (molto ingrandita).

riori sono pure molto ingrossate e servono al salto. Negli insetti acquaiuoli (Dytiscus, Hydroporus, Hydrophilus fra i Coleotteri; Belostoma, Notonecta fra i Rincoti), le zampe posteriori e qualche volta anche le mediane si modificano in modo da pigliare la forma di remi, si appiattiscono cioè e si rivestono lateralmente di una serie di peli foggiati a mo' di laminette, come ho già detto. 
Le zampe, dirò in ultimo, possono atrofizzarsi, ed anche mancare al tutto in seguito al non uso, ed io ho già avuto occasione di parlare delle principali forme apode che si incontrano nei vari ordini degli insetti.

Veniamo ora a parlare delle appendici dell'arco dorsale del mesotorace e del metatorace, vale a dire delle ali.

Le ali considerate nel loro complesso si possono considerare costituite ciascuna da un sacco cutaneo appiattito di cui le due parti si sono saldate fra loro dal lato interno in modo da formare una sola lamina. La saldatura non è tuttavia completa in tutti i punti dell'ala e rimangono liberi nello spessore dell'ala stessa degli spazii canaliformi più o meno numerosi, nei quali scorrono vasi tracheali, e intorno ai quali si accumula principalmente il tessuto chitinoso. Questi spazii vuoti costituiscono quelle parti delle ali che si chiamano nervature.

Le nervature variano moltissimo e nel numero e nella forma e nella disposizione, e il loro studio ha grande importanza nella entomologia sistematica.

Le ali sono per lo più fatte da un tessuto sottilissimo e trasparente come è il caso pei Ditteri, per gli Imenotteri e pei Nevrotteri. Noterò di passaggio che negli insetti di questi ordini le ali presentano spesso bellissimi colori prodotti da fenomeni d'interferenza luminosa.

Nei Coleotteri il tessuto chitinoso si inspessisce molto nel primo paio di ali e riempiendo tutte le areole lasciate dalle nervature, dà alle ali stesse una consistenza coriacea. Il primo paio d'ali dei Coleotteri piglia per la sua speciale struttura il nome di elitrè. Ciò che si è detto pel primo paio di ali dei Coleotteri si può ripetere pure per molti Rincoti e per qualche Ortottero.

Delle varie colorazioni delle ali membranose e delle elitre, ho già parlato.

Le ali negli insetti non sono mai più di quattro, possono essere due sole, le altre due essendo rudimentali, o mancare affatto.

Hanno quattro ali gli Ortotteri, i Nevrotteri, i Rincoti, i Coleotteri, gli Imenotteri ed i Lepidotteri.

Ne hanno due sole (il secondo paio è rudimentale al tutto ed è rappresentato dai bilancieri) i Ditteri. 
Tutti gli ordini di insetti ci offrono esempi di specie prive intieramente di ali. La mancanza di queste parti si spiega colle leggi del non uso (1).

Col non uso si spiega pure la mancanza delle ali membranose in molti Coleotteri, soprattutto carabici, ed in qualche caso anche il saldarsi fra loro delle elitre (Cycrhrus).

Lo stesso si dirà per le forme attere ed apode degli altri ordini.

Nello stesso modo possiamo renderci ragione della mancanza degli occhi che si osserva in molti insetti.

Fra i Coleotteri si sono descritte al giorno d'oggi oltre a cento e sessanta specie cieche, le quali tutte vivono in fondo a buie caverne (2).

Mancanza più o meno compiuta di occhi si incontra pure nel Cephalocteus histeroides Linn., ed in varie specie di Pediculidi e Mallofagidi fra i Rincoti; nel genere Typhlopona West. fra gli Imenotteri. Fra i Ditteri troviamo i generi Braula (B. caeca Nitz, parassita delle api) e Nycteribia (parassita

(1) Per dare un'idea delle modificazioni che il non uso di certe parti produce nelle parti stesse, riferirò il brano seguente dell'Origine delle Specie del Darwin: — "Il signor Wollaston ha scoperto questo fatto nótevole, che cioè sopra le 500 specie di Coleotteri che abitano l'isola di Madera, 200 hanno le ali così difettose che non possono servirsene, e che dei 29 generi che sono propri a quest'isola, non meno di 25 hanno tutte le loro specie in queste condizioni. Vari fatti mi hanno condotto a pensare che l'atrofia più o meno compiuta dell'organo del volo in $\mathrm{m}$ così grande numero di Coleotteri di questa località debba essere il risultato della scelta naturale combinata cogli effetti del difetto di esercizio di quell'organo. È stato osservato a questo proposito che in vari luoghi i Coleotteri sono spesso trasportati dal vento in mare dove periscono. Ora il signor Wollaston ha osservato che i Coleotteri di Madera stanno nascosti fino a tanto che il rento cessa, e che la proporzione delle specie sprovviste di ali è maggiore nelle isole deserte ed esposte ai venti di mare che non a Madera stessa........ Negli insetti di Madera i Coleotteri antofili ed i Lepidotteri che debbono per forza far uso delle ali per procurarsi il nutrimento invece di averle ridotte le hanno al contrario molto sviluppate».

(2) Pochi relativamente erano in principio del corrente secolo gli artropodi ciechi conosciuti. Ma negli ultimi trent'anni, se ne scopersero un grande numero e siamo debitori di queste scoperte alle accurate ricerche fatte nelle caverne. Basti il dire che dall'anno 1844 in cui dallo Sturm si pubblicò il primo Anophthalmus (A. Schmidtii Sturm) si contano al giorno d'oggi oltre cinquanta specie di questo genere tutte cavernicole, o per lo meno ipogee. 
dei pipistrelli). Nei Strepsitteri finalmente sono cieche le femmine delle specie dei generi Xenos, Stylops, ecc.

Sempre colla stessa legge del non uso ci è dato di spiegare la mancanza della bocca, nelle specie del genere Oestrus fra i Ditteri, nei maschi delle specie del genere Coccus e negli individui sessuati della Phylloxera vastatrix (esiste la bocca negli individui partenogenitici) fra i Rincoti e l'essere la bocca stessa al tutto rudimentale ed inservibile in molti Bombiciti (Lepidotteri), in molti Friganidi (Nevrotteri) e in molte Efimere (Pseudo-Ortotteri.

Nello stesso modo noi possiamo trovare una ragione della mancanza dell'ano nelle specie del genere Oestrus (si trovano tuttaria ancora vestigia di tubo intestinale) e nelle larve delle api, di molti altri aculeati e di vari Imenotteri parassiti (1).

Riguardo poi all'addome dirò che gli ultimi segmenti presentano in qualche caso (Podura) una sorta di coda articolata che può ripiegarsi al disotto dell'addome stesso e scattare a guisa di una molla servendo cosi alla locomozione che in questo caso si fa per salti.

Delle appendici poi risultanti dalla trasformazione degli ultimi segmenti addominali e costituenti l'apparato copulatore esterno parlerò trattando degli organi della riproduzione.

Alcune fra le parti sopra menzionate del dermascheletro presentano non infrequentemente delle anomalie e delle asimmetrie più o meno importanti.

Le asimmetrie che osservansi negli insetti si possono dividere in due categorie, vale a dire, in asimmetrie costanti ed in asimmetrie accidentali.

Costanti diconsi quelle che si incontrano in quasi tutti gli individui di una specie e che vengono trasmesse ereditariamente. Presentano asimmetrie di tal sorta fra i Coleotteri, ad esempio, l'Hister inaequalis Fabr., nel quale la mandibola sinistra del maschio è quasi sempre molto più lunga della destra. Fra gli Ortotteri troviamo la Locusta vorax Stoll., in cui non solo la bocca, ma anche le gambe sono fra loro asim-

(1) In questi ultimi i residui della digestione, a quanto pare, sono raccolti in fondo al ventricolo e vengono poi espulsi dalla bocca nel passaggio dallo stato di larva a quello di ninfa. 
metriche. In molti Grilloidei ed in molti Locustini l'organo stridulante del maschio è asimmetrico. Nel genere Heterotoma (Ortotteri Tisanuri), le antenne sono quasi sempre asimmetriche e variano da due a cinque articoli.

Asimmetrie accidentali sono quelle che si osservano solo a

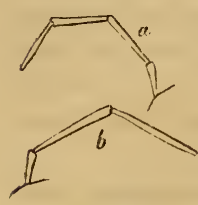
caso in qualche individuo di una data specie, ma non sono caratteristiche della specie stessa.

Troppo lungo sarebbe l'enumerare tutte le specie di insetti in cui sono stati osservati casi di asimmetria, casi che si incontrano il più delle volte $o$ nelle antenne o nelle appen-

Fig. 26.

Dieuches luscus. dici boccali o nelle ali. Si consulti a questo proposito :

G. Krantz. - Ueber missgebildete Kaefer deutsche Entomol. Zeit. XXI, 187\%, p. 58, tav. I, n. II.

L. Camerano. - Deserizione di alcuni insetti mostruosi, ecc. Atti della R. Acead. delle Scienze di Torino, vol. XIV, 1878.

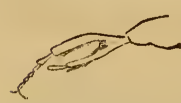

Fig. 27. Chlaenius holosericeus.

Sono pure non infrequenti negli insetti altre sorta di mostruosità e sopratutto quelle che provengono dallo sdoppiarsi di un organo. Questo genere di anomalie si osserva ordinariamente o nelle antenne o nellé zampe.

Anche negli insetti, come in quasi tutti gli altri animali, si

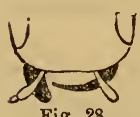

Fig. 28.

Ocypus similis (mandibola destra
mostruosa).

hanno casi di albinismo e casi di melanismo. Intorno a queste e ad altre mostruosità che, a quanto pare, dipendono da cause che agiscono durante la metamorfosi, non abbiamo fino ad ora cognizioni molto sicure. Si consulti a questo proposito:

A. Barthélemy. - Sur les monstruosités naturelles et provoquées chez les Lépidoptères. Ann. de Sc. Nat., 5a ser., I, 1864. 


\section{Bibliografia.}

Straus. Burmeister. Gegengaur. Kirby e Spence. Bronn. - Op. citat.

Lacaze Dethiers. - De l'armure génitale des Insectes, Ann. sc. nat., serie 3a, 1849-1853, vol. 12-14-17-18-19.

Aunouis. - Recherches anatomiques sur le thorax des animaux articulés et celui des insectes hexapodes en particulier. Ann. Sc. Nat., $1^{\text {a }}$ ser., 1824.

- Anatomie comparative des parties solides des Insectes. Ann. gén. d. scienc. phys., 1820, vol. VII.

LA TREILle. - De la formation des ailes des Insectes, ecc. Ann. gén. des sciences phys., 1819.

Newmans. - Osteology or External anatomy of Insects. Ann. Sc. nat., $3 \mathbf{3}$ ser., 1853, vol. XIX.

NEWPORT. - Insecta. Todd's Cyclopædia, t. II, p. 918.

LA TREILLE. - De quelques appendices particuliers du thorax de divers Insectes.

Mém. du Muséum, vol. VII, pl. I, 1821. 


\title{
FUNZIONI DI RELAZIONE
}

\author{
LOCOMOZIONE.
}

La vita di relazione è negli insetti molto attiva, gli organi dei sensi e della locomozione sono molto sviluppati e la divisione del lavoro fisiologico è portata molto oltre. Per questo rispetto anzi gli insetti sono superiori agli altri invertebrati.

Cominciamo dalla locomozione. Gli insetti camminano, saltano, nuotano e volano e talora anche strisciano. Ecco riassunte in poche parole le varie maniere di locomozione degli insetti. Noi abbiamo già veduto con quali organi gli insetti possono attuare queste varie maniere di locomozione. Ci rimane a dire dei muscoli e del modo in cui agiscono le varie parti costituenti gli organi stessi.

Siamo ben lontani oggidi dall'avere cognizioni sicure intorno alla struttura dei muscoli degli insetti. Una cosa tuttavia è sicura, ed è che negli insetti il sistema muscolare è in generale molto sviluppato (e ciò è in rapporto colla celerità e colla forza dei loro movimenti), e che i muscoli degli insetti si differenziano da quelli dei vertebrati per avere le loro fibre non riunite in fasci, e quindi facilmente separabili le une dalle altre. Negli insetti, come pure nei crostacei e negli aracnidi, i muscoli sono striati e i loro fasci elementari presentano secondo il Leydig un canale centrale, nel quale una serie di nuclei forma una colonna serrata. I muscoli degli insetti sono per lo più bianchicci e privi di colore; fanno eccezione tuttavia i muscoli toracici di molte specie, i quali sono di color giallo-bruno.

Negli insetti i muscoli sono molto numerosi, sempre più numerosi nell'insetto perfetto che non nella larva. Il loro numero 
tuttavia non è cosi grande come credeva il Lyonnet, il quale nella larva del Cossus ligniperda annoverò 4061 muscoli. Il Lyonnet considerò come muscoli le fibre elementari che, siccome ho detto, sono negli insetti facilmente separabili le une dalle altre.

Nell'età adulta i muscoli del capo, nei Coleotteri, ed in generale in tutti gli insetti nei quali il capo è incassato nel torace, sono in numero di quattro paia, vale a dire, un paio di muscoli estensori superiormente, due paia di muscoli flessori inferiormente, l'uno robusto, l'altro un po' più debole, e finalmente un paio di muscoli laterali. Negli Imenotteri $e$ in tutti quegli insetti in cui il capo non è incassato nel torace, i muscoli sopradetti esistono allo stato rudimentale e talvolta anche qualche paio manca al tutto.

Nel capo poi si debbono ricordare tutti i muscoli destinati ai movimenti delle varie appendici articolate: antenne, palpi, mandibole, mascelle, ecc. Le antenne poi oltre a tre muscoli principali, esterno, flessore, elevatore, hanno pure una serie di piccoli muscoli fra i vari articoli, destinati ai movimenti degli articoli stessi. Notero in ultimo che il massimo numero dei muscoli si trova negli insetti masticatori, il minimo invece nei succiatori.

Il torace è la parte del corpo che contiene i muscoli più voluminosi e piú robusti. Una parte di questi sono destinati ai movimenti dei tre anelli toracici, gli altri sono incaricati dei movimenti delle zampe e delle ali. Le ali hanno ciascuna due muscoli estensori ed un solo flessore. Le zampe ne hanno invece un numero molto maggiore $\theta$ ciò in ragione dei movimenti svariatissimi che queste parti possono eseguire.

Meno sviluppati che non nelle due parti del corpo or ora menzionate sono i muscoli dell'addome.

L'addome si unisce al torace per mezzo di quattro muscoli più o meno robusti, i quali si riducono ad uno solo quando l'addome è unito al torace per mezzo di un peduncolo. Un'altra serie più o meno numerosa di muscoli unisce fra loro i vari segmenti addominali, muscoli che nei casi di completa saldatura dei segmenti (qualche Coleottero) sono allora appena rudimentali. L'addome, finalmente, contiene pure una serie di muscoli destinati ai visceri, ed una serie di muscoli destinati 
ai movimenti dell'apparato copulatore esterno negli insetti in cui questo apparato è sviluppato.

La disposizione dei muscoli negli insetti allo stato larvale varia secondo che gli insetti hanno metamorfosi compiuta od incompiuta. Nelle larve di insetti a metamorfosi incompiuta i muscoli hanno una disposizione analoga a quella dell'insetto adulto, salvo naturalmente i muscoli delle ali che non si sviluppano che nell'ultimo stadio. Nelle larve invece di insetti a metamorfosi compiuta, la disposizione dei muscoli è assai diversa da quella che si osserva nell'insetto perfetto, e ricorda la disposizione dei muscoli addominali. Il loro numero tuttavia è più grande, e son disposti a strati in modo che la direzione delle fibre è diversa nei vari strati. Ora le fibre hanno una direzione longitudinale, ora invece una direzione obliqua.

Nella larva si trova pure un sistema di muscoli destinati a muovere le zampe toraciche. Una speciale serie di muscoli finalmente, mediocremente sviluppati, mette pure in movimento, quando vi sono, le zampe addominali o false zampe.

La maniera più frequente di muoversi che si osserva negli insetti è il camminare. Gli insetti possono correre rapidamente 0 muoversi a stento. Ora la locomozione è ridotta a semplici movimenti di strisciamento simili a quelli dei serpenti, come è il caso di molte larve di Ditteri e di Lepidotteri. Ora peli e spine speciali agevolano la locomozione, ora finalmente appendici articolate determinate rendono il camminare dell'insetto piú rapido è più sicuro. Sono in generale buoni camminatori quegli insetti che volano poco (Coleotteri, soprattutto le specie appartenenti alla famiglia delle Cicindele e dei Carabici). Cattivi camminatori invece quelli che hanno le ali molto sviluppate (Lepidotteri).

Varie sono le maniere in cui gli insetti possono eseguire il salto. Ora il salto si fa per mezzo di un movimento generale del corpo come si osserva in parecchie larve di Ditteri, ora per mezzo dello scattare delle zampe posteriori, le quali sono in questo caso molto più sviluppate che non le mediane e le anteriori (molti Ortotteri e molti Coleotteri), ora per mezzo di organi appositi diversi dai citati, quali sono le appendici addominali delle Podure fra gli Ortotteri. Un'ultima maniera di salto finalmente è quella che si osserva in parecchie specie 
di Elateridi, salto che si compie per mezzo del protorace e del mesotorace (1).

Le ultime due maniere di locomozione degli insetti, il nuoto ed il volo, appartengono ad una stessa categoria di fenomeni. Gli organi solo si modificano col cambiarsi del mezzo in cui si compie il movimento. La leva che fa da propulsore deve essenzialmente offrire una larga superficie nel momento della spinta, e invece ripiegarsi, o presentare una stretta superficie, durante lo slancio. L'azione di questa leva naturalmente deve essere più potente quando l'insetto si muove nell'aria che non quando si appoggia sull'acqua. In molti animali ed anche negli insetti stessi, come ha osservato recentemente il Lubbock (2) nella Polynema natans fra gli Imenotteri, le ali possono pure servire al nuoto.

Il nuoto, come il salto, puó compiersi negli insetti in varie guise, or con movimenti dell'intiero corpo, come si osserva in molte larve di Ditteri e in qualche larva di Pseudonevrotteri, ora per mezzo delle branchie esterne che fanno uffizio di natatoie, come nelle larve delle friganee, ora per mezzo delle zampe posteriori e qualche volta anche delle mediane che sono foggiate a mo' di remi (molti Coleotteri e vari Rincoti) (3). Le

(1) Questi insetti fanno uso del salto non per trasportarsi da luogo a luogo come è il caso per tutti gli altri insetti saltatori, ma bensì semplicemente per rimettersi sulle loro gambe quando sono caduti sul dorso, cosa che avviene loro assai frequentemente essendo lor costume, per sfuggire a qualche pericolo, di lasciarsi cadere a terra come corpo morto dai trouchi degli alberi contro ai qualiper lo più sogliono stare. Questi insetti, quando sono rovesciati sul dorso, non potendo per rimettersi in piedi far uso delle zampe, che sono relativamente molto corte, ricorrono al salto. Lo sterno del loro mesotorace presenta una cavità nella quale viene a mettere capo una pretuberanza corrispondente che si trova sul protorace. Quando sono coricati sul dorso, alzano la parte mediana del loro corpo in modo da formare un arco colla concavità che guarda il suolo, poi con un movimento rapidissimo ricacciano la parte rialzata contro il suolo. L'urto che ricevono in questo nod̉o concede loro di innalzarsi ad una certa altezza in aria. Nel salto l'animale si volta e ricade sulle sue zampe.

(2) « On two new aquatic Hymenoptera (Trans. Linn. Soc. 1863; t. XXIV).

(3) Fra i Coleotteri che fanno vita acquatica, alcuni non nuotano, ma camninano semplicemente sul fondo o strisciano alla superficie dell'acqua (Berosus, Helophorus). Fra i Rincoti i generi Gerris, Halobates, ecc., si muovono pure rapidamente strisciando alla superficie dell'acqua. 
larve delle libellule finalmente si muovono nell'acqua per mezzo di impulsi che ricerono cacciando fuori rapidamente l'acqua che esse introducono nel loro addome per mezzo dell'apertura anale.

Caratteristica degli insetti è l'ultima maniera di locomozione di cui ci rimane a dire, il volo.

L'energia e la rapidità del volo dipendono non solo dalla forza muscolare dell'insetto, ma anche dalla posizione, dalla forma e dalla consistenza degli organi del volo. I Coleotteri, ad esempio, nei quali il primo paio di ali è duro e resistente, sono poco volatori, mentre invece gli Ortotteri, i Ditteri, gli Imenotteri e i Lepidotteri hanno volo rapidissimo (1).

La forza muscolare degli insetti, dirò in ultimo, è molto grande ed è proporzionatamente maggiore di quella dei vertebrati. Si consultino a questo proposito gli scritti del signor F. Plateau, Bulletin de l'Academie royale de Belgique. Bruxelles, 1865, vol. XX, e 1866, vol. XXII.

(1) Secondo le ricerche del Marey, il numero dei battiti delle ali, in un minuto secondo, considerando le oscillazioni intiere, è di 330 nella mosca comune, 190 nell'ape, 28 nella Libellula, 9 nella comune Cavolaia. 


\section{Bibliografia.}

Milne Edwards, Gegenbaur, Straus, Bronn, ecc., op. eitate.

Aмгсі. - Sulla fibra muscolare. Nuovo cimento, 1859, vol. IX.

G. A. WAgNer. - Ueber die Muskelfaser der Evertebraten. Arch. für Anat., 1863, p. 211.

Schneider. - Ueber die Muskeln der Würmer. Arch. für Anat., 1864, p. 590.

Kunkel. - Sur le développement des fibres musculaires striées chez les Insectes. Comptes rendus de $\vec{C}$ Acad. des Sciences, 1872, vol. LXXV, p. 359.

IммаN. - On the power by which Insects are enabled to adhere to smooth perpendicular surface. British Associat. Trans., 1854, p. 109.

Krarup-Hausex. - Essai d'une théorie du vol des Oiseaux, des Chauves-Souris et des Insectes. Copenhagues, 1869.

Plateau. - Reflexion et expér. sur le vol des Coléoptères. Bibl. univ. de Genève, 1869.

Chabrier. - Essai sur le vol des Insectes, 1822.

- Explication du vol des Insectes. Ann. des Scienc. Nat., 1829, vol. XVI.

Pettigrew. - La locomotion, 1874. 


\title{
FUNZIONI DI RELAZIONE
}

\author{
SISTEMA NERVOSO.
}

Per molto tempo il sistema nervoso venne negato agli insetti. Il Malpighi e lo Svammerdam furono i primi a riconoscerlo ed a descriverlo in qualche specie; ulteriori ricerche poi hanno fatto conoscere la sua esistenza e in parte anche la sua struttura in tutti o per meglio dire in quasi tutti gli insetti. Dico quasi tutti, imperocchè ultimamente il Leon Dufour (1) - annunziò la mancanza del sistema nervoso in una specie di Nevrottero appartenente al genere Nemoptera. La cosa tuttavia meriterebbe di essere verificata (2).

Negli insetti dunque, come negli animali superiori, noi troviamo pure quel sistema di organi incaricati di regolare le funzioni, di raccogliere le sensazioni ricevute dai vari organi posti alla periferia, o dagli organi interni, e di trasmetterle sotto forma di volizioni agli organi stessi.

Secondo le ricerche moderne, negli insetti esistono, come negli animali superiori, due sistemi nervosi, un sistema nervoso cioè della vita animale ed un sistema nervoso della vita vegetativa.

Il sistema nervoso della vita animale ha un piano di struttura al tutto diverso da quello degli animali superiori. Esso è costituito da un cingolo esofageo e da una serie di gangli uniti

(1) * Note sur l'absence dans le Nemoptera lusitanica d'un système nerveux appréciable ». Ann. des Scienc. Nat., $4^{\text {a }}$ ser., vol. IV, 1855.

(2) Reca meraviglia il fatto che nessuno, per quanto io so, abbia cercato, potendo avere disponibile fresca la specie sopra nominata, di verificare un fatto così importante. 
fra loro da cordoni nervosi disposti nella parte inferiore del corpo al disotto degli organi della nutrizione. Il cervello e la catena gangliare degli insetti non possono, anatomicamente parlando, in verun modo essere paragonati al cervęllo ed al midollo spinale dei vertebrati (come venne fatto da molti autori) essendo il punto di partenza di questi organi nelle due serie di animali, come ha dimostrato il Gegenbaur, al tutto diverso.

Considerato nella sua forma più semplice, il sistema nervosi) degli insetti ci presenta due serie parallele di gangli corrispondenti a ciascun segmento o zoonito. Questa disposizione, che
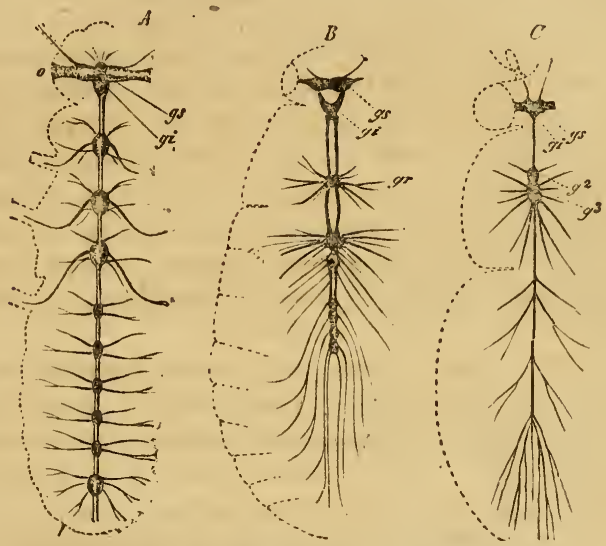

Fig. 29. Sistema nervoso di vari insetti.

$A$ termite $-B$ ditisco - $C$ mosca. - gs gangli cerebroidi coi nervi che vanno alle antenne ed agli occhi - gi gangli sottoesofagei - gr, $g^{2} g^{3}$ gangli della catena ventrale saldati fra loro - a gangli toracici divisi coi rami nervosi che si distribuiscono alle estremità inferiori - 0 occhi e nervi che a questi arrivano dai gangli cerebroidi.

si potrebbe dire primordiale, non si osserva ora che nello stato larvale delle forme più semplici, imperocchè in seguito, col centralizzarsi delle varie parti del corpo, anche i gangli si sono riuniti fra loro, non solo trasversalmente (il che si osserva in una maniera più o meno evidente in tutte le forme di insetti anche nelle più semplici), ma anche longitudinalmente in modo che la loro disposizione ed il loro raggruppamento corrisponde negli insetti più perfezionati precisamente alle tre 
parti principali del corpo: capo, torace, addome. Il grado di centralizzazione quindi del sistema nervoso non è molto grande nella larva, cresce nella ninfa, cresce ancora e raggiunge il massimo suo grado nell'insetto perfetto.

Nel capo trovasi il cingolo esofageo, il quale è costituito superiormente da una massa biloba formata dalla riunione di due gangli che pigliano il nome di gangli cerebroidi: questi gangli si uniscono per mezzo di due commessure nervose al ganglio sottoesofageo che dà principio alla catena gangliare ventrale. In qualche caso, si noti, $i$ due gangli cerebroidi si fondono insieme così intimamente che non appare più traccia esternamente della loro primitiva divisione. Il volume del ganglio o meglio dei gangli cerebroidi, comparato con quello del corpo, secondo le ricerche del Dujardin (1), varia molto negli insetti, nell'ape è eguale ad un centosettantaquattresimo del volume totale del corpo, in una specie di Icneumonide invece è solo un quattrocentesimo del volume del corpo. Sopra questo argomento tuttavia sono necessarie altre e più numerose osservazioni.

Dai gangli cerebroidi finalmente, partono i nervi, per lo più molto sviluppati, che vanno agli occhi, alle antenne ed al labbro superiore.

Intorno alla struttura intima dei gangli cerebroidi le nostre cognizioni sono molto scarse. Credo utile a questo proposito di ricordare colle parole stesse dell'autore un fatto osservato dal Dujardin (2):

«Si l'on verse sur le cerveau frais, mis à nu, le liquide destiné a le consolider, il ne devient pas uniformément blanc et opaque, mais on voit paraître d'abord des traces de circonvolutions, qu'on achève d'étudier plus facilement quand l'immersion dans le liquide est assez prolongée. On voit alors sur le sommet du cerveaux des Hyménoptères, vers l'endroit qui

(1) - Sur le système nerveux des Insectes 3. Ann. des Scienc. Nat., 3a serie, vol. XIV, 1850.

(2) Op. citata.

Per ciò che è della struttura istologica dei gangli e dei nervi degli insetti, si consultino principalmente le opere seguenti:

Helmнolтz: - De Fabrica systematis nervosi Evertebratorum, Besol., 1842.

Lexplg. - Handb. d. Verg. Anat., I. p. 214. 
correspond aux stigmates, des circonvolutions régulières, plus ou moins distinctes, comparables à celles des mammifères. Si l'on enlève ensuite les portions de substance pulpeuse ou corticale qui masquent ordinairement ces circonvolutions, on finit par les voir tout-à-fait à nu, et l'on reconnait qu'elles appartiennent à une substance encore plus blanche et plus consistante ».

La catena gangliare ventrale risulta costituita dal ganglio sottoesofageo, dai gangli toracici (1) e dai gangli addominali.

I gangli toracici sono in numero di tre paia, uno pel protorace, uno pel mesotorace ed uno pel metatorace. Dal primo partono i nervi che vanno al primo paio di zampe, dal secondo quelli che vanno al secondo paio di zampe ed al primo paio di ali, dal terzo quelli che vanno all'ultimo paio di zampe ed al secondo paio di ali. L'origine di questi nervi è invariabile in tutti gli insetti.

Nello stato larvale di molti insetti i tre gangli toracici sono separati fra loro, ad esempio nel bruco del baco da seta, in molti insetti invece allo stato perfetto questi si uniscono in parte od intieramente fra loro (ad esempio nella farfalla del baco da seta).

Negli Ortotteri, nei Nevrotteri e nei Coleotteri il ganglio del protorace non si salda coi seguenti. Nell'ape adulta il ganglio del protorace rimane pure isolato, ma si saldono invece fra loro gli altri due.

La fusione dei gangli in una massa sola è indizio di superiorità e non si osserva che nelle forme più perfezionate degli insetti.

Il numero dei gangli addominali non supera nella massima parte dei casi il numero di nove. Nelle larve per lo più sono distinti fra loro; negli insetti adulti si uniscono fra loro a costituire una sola massa più o meno allungata che molte volte si salda col ganglio del metatorace, come è il caso, ad esempio, nella Melolonta adulta.

(1) Il ganglio sottoesofageo risulta pure formato dalla fusione di due gangli e si unisce anteriormente ai gangli cerebroidi e posteriormente col primo ganglio toracico e dà origine ai nervi destinati al labro inferiore, alle mascelle ed alle mandibole. 
Da quanto precede appare adunque che negli insetti dal cervello partono principalmente i nervi degli organi dei sensi e dalla catena gangliare, quelli del movimento e della sensitività.

Il sistema nervoso della vita vegetativa, osservato per la prima volta dallo Swammerdam, poscia dal Lyonnet, e studiato ultimamente soprattutto dal Newport e dal Blanchard, comprende una serie di piccolí gangli e di nervi che da questi derivano disposti intorno ai gangli cerebroidi. I nervi del sistema nervoso della vita vegetativa si possono dividere in tre categorie, vale a dire, nervi stomacali, nervi del vaso dorsale o dell'apparato circolatorio e nervi delle trachee o dell'apparato respiratorio.

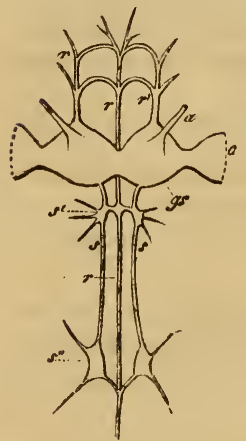

Gangli cerebroidi e sistema nerroso viscerale del Bombyx Inori - gs gangli cerebroidi - $a$ nervo che va alle antenne - o nervo ottico $-r$ ramo impari del sistema nervoso viscerale $-r$ sue radici dai gangli cerebroidi $-s$ nervi pari del sistema viscerale $-s s^{\prime \prime}$ loro rigonfiamenti gangliari.

Fig. 30 .

Il sistema nervoso stomacale consiste in una catena di piccolissimi gangli, ora semplice ora doppia, almeno nella sua parte posteriore, collocata sulla faccia superiore del canal digerente.

I gangli ed i nervi del sistema circolatorio e del sistema respiratorio sono collocati ai lati dell'esofago e mandano dei filamenti finissimi al cervello, al vaso dorsale ed alle trachee.

Finalmente entra pure a far parte del sistema nervoso della vita vegetativa una serie di piccolissimi gangli che è collocata sopra alla catena gangliare ventrale. Queste due catene gangliari si uniscono fra loro per mezzo delle anastomosi che si fanno fra i nervi che da esse derivano.

La prima parte del sistema nervoso della vita vegetativa, 
venne, considerando le sue funzioni, dal Newport paragonata ai nervi pneumogastrici degli animali superiori. La seconda parte, che è quella destinata alla innervazione degli orifizi respiratori, di parte del canal digerente e degli organi della riproduzione, venne dal Blanchard paragonata al gran simpatico degli animali superiori.

\section{Bibliografia.}

Ileydig, Milne Edwards, Gegenbaur, Straus-Durckheim, L. Dufour. - Opere citate.

Brandt. - Remarques sur les nerfs stomato-gastriques. Ann. des Scienc. Nat., $1^{\text {a }}$ serie, 1828 , vol. XIV.

BLANCHARD. - Recherches anat. et zoologiques du système nerveux des Insectes. Ann. des Scienc. Nat., $3^{\text {a }}$ serie, 1846, vol. V.

- Métamorphose des Insectes. Paris 1868.

- Du grand sympathique chez les animaux articulés. Ann. des Scienc. Nat., $4^{\mathrm{a}}$ ser., X. 1858.

NewPort. - Art. Insecta (Todd's Cyclop., vol. II, p. 950, fig. 409).

- On the nervous System of the Sphinx Ligustri. Philos. Trans. 1832, T.122. - 1834, T. 134.

- On the structure, relations and development of the nervous and circulatory Systems, ecc. Philosoph. Transact., 1843.

S. Müller. - Ueber ein eigenthümliches, dem Nervus sympathicus analoges Nervensystem der Eingeweide bei den Insekten. Nov. Act. Acad. Leopold. Carol. XII, 1825.

A. Yersin. - Recherches sur les fonctions du système nerveux dans les animaux articulés. Bullett. de la Soc. Vaudoise d. Scienc. Nat., V, 1856. 


\title{
X. \\ FUNZIONI DI RELAZIONE
}

\author{
ORGANI DEI SENSI.
}

La vita di relazione degli insetti, molto estesa, importa naturalmente un grande sviluppo ed una grande squisitezza degli organi dei sensi; giá da lungo tempo insigni naturalisti ed anatomici diedero opera allo studio degli organi dei sensi degli insetti, e molto scrissero sopra questo argomento. Le nostre cognizioni tuttavia in proposito sono al giorno d'oggi ancora poche e poco precise.

Cominciamo dal senso del tatto, fra tutti i sensi quello che manca più difficilmente negli animali e ćhe primo si incontra salendo nella scala zoologica.

Negli insetti il tatto, per quanto almeno se ne sa oggi, è in generale ben sviluppato. Nello stadio di larva, quando l'integumento è molle, le terminazioni nervose tattili sono collocate nello spessore della pelle stessa e quindi il tatto è sparso per tutto il corpo. In questo caso la sensitività tattile della pelle viene per lo più molto accresciuta dalla presenza di un grande numero di peli (che d'ordinario si osservano sul corpo delle larve, ora disposti a ciuffi, ora a striscie, ora ricoprenti l'intera superficie del corpo, ora semplici, ora invece spinosi, ora corti, ora invece assai lunghi, ecc.) che sono alla loro base in diretto rapporto colle terminazioni nervose della pelle stessa.

Nell'insetto adulto la sensitività tattile si centralizza e si riduce in organi determinati, e quindi, sempre per la legge della divisione del lavoro fisiologico, si fa più perfetta e squisita.

La pelle dell'adulto è in generale dura ed è priva di ter- 
minazioni nervose speciali, e, salvo il caso degli insetti ciechi (soprattutto coleotteri), non ha peli tattili.

A quanto pare, il tatto negli insetti è localizzato nelle antenne, nei palpi ed anche, in qualche caso, nei tarsi anteriori rivestiti da numerosi peli tattili.

Dai gangli cerebroidi e dai gangli sottoesofagei partono parecchi filamenti nervosi, i quali vanno a far capo alle antenne ed ai palpi, e presentano numerose terminazioni gangliformi. Queste ultime sono in diretta comunicazione con una categoria speciale di produzioni cutanee chiamate bastoncini tattili.

Terminazioni nervose con bastoncini tattili di una mosca. - $n$ nervo - $g$ rigonfamenti gangliari $-s$ bastoncini tattili $-c$ peluzzi della cuticola.

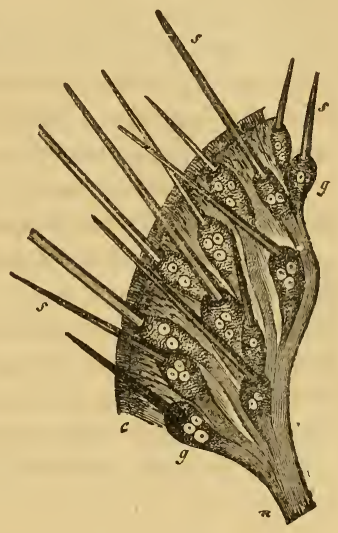

Fig. 31 .

I bastoncini tattili, oltre al trovarsi più o meno numerosi sulle antenne e sui palpi, si trovano pure in molti casi sui tarsi. Forse i peli costituenti le spazzolette copulatrici dei maschi servono pure come organi di tatto.

Negli insetti succiatori, Ditteri, ecc., molto probabilmente la proboscide funziona anche come organo di tatto.

Secondo certi autori, in fine, i filamenti spesso lunghissimi che si trovano sull'ultimo segmento addominale servirebbero pure in certi casi come organi di tatto. La cosa tuttavia non è ancora ben sicura e richiede nuovi studi.

L'ipotesi sopra enunciata, che le antenne od i palpi funzio- 
nino come organi di tatto, è senza dubbio molto probabile. Infatti, studiando l'insetto mentre è vivo, noi vediamo che egli muove continuamente queste parti e spingendole innanzi a sè cerca di riconoscere i luoghi e gli oggetti. E col contatto reciproco delle antenne, come tutti sanno, che le api $\theta$ le formiche si comunicano certe sensazioni speciali. Molto probabilmente la squisitezza del tatto è negli insetti molto grande e per mezzo di questa essi possono ricevere dai vari oggetti un grande numero di sensazioni diverse e di cui noi non abbiamo idea (1).

Negli insetti il gusto esiste certamente, come ce lo dimostra il fatto dello scegliere che essi fanno il loro cibo e l'avidità che in generale essi mostrano per le sostanze zuccherine. In qualche caso, come ad esempio in molte larve, esso risiede nell'ipofaringe che è molle ed umida. Negli insetti succiatori esso trovasi, a quanto pare, nella proboscide.

Il gusto è più squisito negli insetti succiatori e nelle specie fitofaghe che non nelle specie carnivore.

L'odorato non solo esiste negli insetti, ma vi è molto squisito. Esso è forse il più sviluppato fra tutti i sensi, ed è quello che ha maggior importanza nella vita degli insetti. Ma se non vi è dubbio intorno all'esistenza dell'odorato, non sappiamo tuttavia con certezza in quale parte dell'insetto esso si trovi.

Si è per mezzo dell'odorato che un grande numero di insetti (ad esempio le mosche, i necrofori, le silfe (2), ecc.),

(1) Io ho osservato a questo proposito che la femmina della Forficula auricularia, la quale, come si sa, ha cura delle uova deposte fino a tanto che non siano sviluppate, tasta di tratto in tratto le uova coi palpi. Osservai pure che quando a bella posta io disperdeva qua e là le uova dapprima riunite in un mucchietto, la femmina andava alla ricerca di esse e prendendole colle mandibole e tastandole coi palpi le ammucchiava di nuovo. Durante questa operazione io vidi ripetutamente la forficola tastare coi palpi un uovo, poi correre ad un altro e questo prendere dopo la stessa manovra e portare colle mandibole al mucchio.

(2) L'odorato in questi insetti è acutissimo e possono anche a distanze grandissime accorgersi della presenza di sostanze animali in putrefazione. Sul terrazzo del Museo Zoologico di Torino frequentemente si trovano nella buona stagione Necrofori, Silfe e Stafilini, venuti, attraversando tutta la città, dai prati o dalle colline circostanti, attirati dall'odore degli animali in putrefazione che si sogliono tenere sui terrazzi per le preparazioni anatomiche. 
vanno alla ricerca del loro nutrimento e delle sostanze nelle quali essi debbono deporre le uova (1).

L'odorato serve pure ai maschi di varie specie, soprattutto di lepidotteri, per ricercare la femmina.

Fra le molte ipotesi emesse intorno alla sede dell'organo dell'odorato negli insetti, ricorderemo le seguenti:

L'Huber credeva che risiedesse nella cavità boccale.

Altri, fra i quali Lehmann, Cuvier, Duméril, Straus-Durckheim, Burmeister, ecc., lo dissero trovarsi o sul margine delle stimme o nelle trachee stesse. Altri in fine considerano come sede dell'odorato le antenne. Quest'ultima ipotesi è quella che forse più si accosta al vero, e la maggior parte dei molti esperimenti che sono stati fatti in proposito tendono pure a confermarla. Il Lefebre ed il Dugès, fra gli altri, hanno dimostrato, sperimentando sopra vespe e sopra la comune mosca della carne, che tagliando le antenne l'insetto rimane insensibile agli odori che prima riuscivangli molesti.

Che gli insetti odano, noi non possiamo porre in dubbio.

Il grillo maschio produce per mezzo di uno speciale apparato fonante un rumore stridulo che è perfettamente inteso dalla femmina, la quale non solo riconosce dal suono il luogo dove è il maschio e vi accorre, ma fa anche una scelta fra i vari cantatori a seconda della dolcezza del loro canto. Ciò che si dice del grillo si deve dire pure, come abbiamo veduto in un precedente capitolo, delle cicale.

Ma se l'udito esiste negli insetti, dove esiste? Ecco ciò che non sappiamo in modo sicuro.

Giovanni Müller e Siebold considerano come organi uditivi certe fossette che sono sul metatorace dei grilli. La cosa pro-

(1) Qualche volta l'odorato trae in inganno gli insetti, come il Roesel pel primo (Insecten Belustigung. Muscarum atque Culicum, tab. IX), ed altri dopo di lui hanno dimostrato (Domérlu, Dissert. sur l'organe de l' Odorat et sur son existence dans Tes Insectes. Magazin encyclopédique, V). Varie piante della categoria degli Arum maudano un odore di carne putrefatta che attira le mosche, le silfe, gli stafilini, ecc., i quali accorrono ingannati dall'odore e affidano alla pianta la loro progenie, la quale è naturalmente destinata a perire per mancanza di nutrimento. Questo fatto non solo è una prova dell'esistenza dell'odorato negli insetti, ma dimostra anche che l'istinto negli insetti non è infallibile. 
babilmente è giusta per ciỏ che è dei grilli e di vari altri ortotteri : infatti si ha in questi insetti una membrana (che piglia il nome di membrana timpanica) tesa come la pelle di un tamburo sopra un cercine chitinoso. Contro alla membrana del timpano viene a collocarsi una vescicola tracheale ed insieme a questa un'espansione nervosa, i nervi della quale hanno terminazioni in forma di bastoncini.

Negli altri insetti, dove cioè non troviamo sul metatorace le cavità or ora menzionate, l'udito risiede molto probabilmente nelle antenne. Ultimamente anzi il signor Berté studiando la struttura delle antenne della pulce comune ha riconosciuto nel segmento terminale di esse una vescicola labirintica, delle cellule fusiformi simili a quelle dell'organo di Corti, e una terminazione speciale del nervo antennale.

L'idea che le antenne (1) siano la sede dell'udito viene confermata e dalle esperienze del Savart, secondo il quale le antenne sarebbero istrumenti atti a contribuire per la loro facoltà vibrante alla percezione dêi suoni, e dal trovarsi nei crostacei, come ha dimostrato il Milne Edwards, un vero apparato uditivo alla base delle antenne.

I lavori di I. Müller, di Leydig, di Claparède, di M. Schultze e di altri hanno fatto progredire di molt́o le nostre conoscenze intorno alla struttura degli organi della vista negli insetti, in modo che il senso della vista degli insetti è quello che ci è meglio conosciuto.

Gli occhi negli insetti sono per lo più ben sviluppati; sono rudimentali o mancano interamente solo nei casi relativamente poco numerosi già citati di metamorfosi regressive.

Gli occhi degli insetti vengono divisi in due categorie, vale a dire in occhi semplici, od ocelli, ed in occhi composti.

(1) Ricorderò le esperienze fatte da A. Mayer intorno all'influenza delle vibrazioni sonore sui peli che si trovano sulle antenne delle zanzare (Researches in acoustic. Edinburg. and Dublin Philosop. Mag., serie $4^{\mathrm{a}}, 1874$. - Experiments on the supposed auditory apparatus of the Cules Musquito. Ann. of nat. hist., serie $4^{\text {a }}$, vol. XV, 1875). Egli osservò che avvicinando all'antenua un diapason che vibrasse in modo di dare la nota $u t, 4$ certi peli vibravano rapidamente mentre altri si stavano tranquilli, e prendendo altri suoni, altri peli entravano in vibrazione. Un fatto analogo venne pure osservato nei crostacei. 
Gli ocelli ridotti alla loro più semplice espressione constano essenzialmente di bastoncini cristallini immersi in una massa pigmentale, di una cornea, di un rigonfiamento lenticuloide e di terminazioni nervose.

Gli occhi composti non sono altro che riunioni di un numero più o meno grande di occhi semplici.

Esaminiamo brevemente le varie parti che entrano a costituire gli occhi composti.

Facendo una sezione di un occhio composto di un insetto, noi troviamo, andando dall'interno verso l'esterno, anzitutto il nervo ottico, il quale è per lo più molto grosso e termina anteriormente in un rigonfiamento gangliare che prende anche il nome di retina gangliare.

La retina gangliare risulta costituita da cellule di varie di-

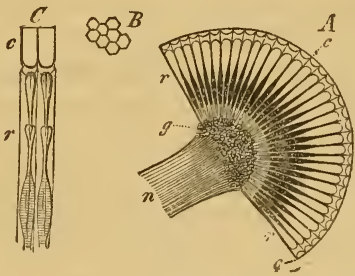

Fig. 32 .

A Sezione schematica di un occhio di artropodo $-n$ nervo ottico $-g$ suo rigonfiamento gangliare $-r$ bastoncini cristallini $-c$ cornea a faccette; ciascuna faccetta sembra, per la sua convessità, abbia nella sua parte interna un organo fatto a mo' di lente. $-B$ faccette viste superiormente $-C-r$ bastoncini cristalliui $-c$ parte lenticolare (Coleottero).

mensioni, di una massa granulare e della sostanza fibrillare, dei nervi ottici; essa presenta vari strati.

Lo strato dei bastoncini cristallini (stratum bacillorum) tiene dietro immediatamente alla retina gangliare, ed ha in questa le sue radici. I bastoncini cristallini negli insetti, per quanto almeno se ne sa, hanno una struttura alquanto complessa. Possiamo distinguere in essi anteriormente un cono cristallino, posteriormente a questo il bastoncino ottico, il quale essendo arvolto quasi intieramente da sostanza pigmentale ed essendo in diretta comunicazione col nervo ottico è la parte dell'occhio più sensibile all'azione della luce. 
Le estremità anteriori dei bastoncini cristallini sono in contatto colla cornea trasparente. Fra questa tuttavia e il cono cristallino si trovano spesso delle masse chiare che hanno strati di varia consistenza e che vennero da qualche autore considerate come cristallini propriamente detti. Morfologicamente, come dice il Leydig, queste parti non sono altro che rigonfiamenti terminali dei bastoncini stessi.

In stretto rapporto coi bastoncini, secondo certi autori, vi sarebbero pure delle fibre muscolari destinate ad agire nel fenomeno dell'adattamento. Queste fibre vengono da autori recenti, fra gli altri Künchel e Chatin, negate.

Si trovano pure intorno ai bastoncini numerosi rami tracheali, i quali dopo aver camminato parallelamente ad essi per un certo tratto si terminano a fondo cieco al disotto delle faccette oculari.

Lo strato chitinoso tegumentale si modifica al disopra dei bastoncini cristallini, diventa trasparente e costituisce una faccetta oculare o cornea, la quale negli insetti per lo più è biconvessa. La biconvessità delle cornee, come ha osservato il Müller, è evidentissima nei Lepidotteri e negli Imenotteri. Le cornee degli insetti hanno la forma di un esagono (1). Esse negli occhi composti sono collocate l'una vicino all'altra e danno all'occhio stesso l'aspetto reticolato caratteristico. In qualche caso (alcuni Ditteri ed Imenotteri) fra cornea e cornea si trovano dei peluzzi, osservati già dallo Swammerdam, più o meno lunghi e più o meno numerosi, che vennero da certi autori paragonati alle ciglia degli animali superiori. Le cornee lucide, quantunque trasparenti, non sono sempre incolore: qualche volta sono o giallognole, o verdognole, o hanno riflessi madraperlacei, ecc.

Il numero delle cornee o faccette che costituiscono un occhio composto è in generale molto grande e varia da 20 (Pselaphus fra i Coleotteri) a 50 (nelle formiche e nel genere Xenos), a 24,058 (Mordelle) (2).

(1) In altri artropodi, ad esempio in molti crostacei, le cornee hanno forma di un quadrilatero.

(2) Nella comune melolonta si contano 8000 faccette. Nel filugello 6236. Nel Cossus Tigniperda 11.300, nella mosea comune 4600, ecc. 
Dando ora un'occhiata complessiva agli occhi composti degli insetti, vediamo che essi sono in generale molto convessi e possono essere o rotondi, o reniformi, od anche, come si osserva nei generi Gyrinus e Tétraops fra i Coleotteri, divisi in due parti distinte.

Negli insetti gli occhi sono sempre immobili e quasi sempre sessili; dico quasi sempre, imperocchè nei generi Achias e Diopsis (Diopsis subfasciata) fra i Ditteri (1), il capo si prolunga lateralmente in due appendici, le quali portano alla loro sommità gli occhi. Un leggiero peduncolo presentano pure gli occhi di qualche Coleottero e quelli dei Ripipteri.

Lo sviluppo degli occhi, come io ho già avuto occasione di notare è variabilissimo ed è in rapporto coi costumi e col genere di vita dell'animale. Gli occhi sono ben sviluppati negli insetti diurni, poco invece nei notturni, rudimentali o mancanti al tutto in quelli che fanno vita sotterranea (2).

In molti casi gli occhi sono più grossi nei maschi che non nelle femmine.

Gli insetti finalmente possono avere o solo ocelli, o solo occhi composti, o le due sorta di occhi ad un tempo.

Nello stato larvale o non vi hanno occhi affatto o vi hanno ocelli. Rari sono i casi in cui nello stato di larva vi siano ad un tempo ocelli ed occhi composti.

Nello stato perfetto hanno per lo più soli ocelli i Tisanuri

(1) Qualche autore tuttavia, fra gli altri il Castelnau, considera come sessili anche gli occhi dei generi citati non essendo i prolungamenti, sopra cui gli occhi sono impiantati, articolati col capo.

(2) Ricorderò a proposito degli insetti mancanti di occhi il fatto seguente menzionato dal prof. Michele Lessona nel suo scritto intitolato: "Dell'Azione della luce sugli animali », Atti della $R$. Accad. delle Scienze di Torino, vol. X, 1874-75; servendomi delle parole stesse dell'A.... «Lo Scotodipmus glaber Baudi, della famiglia dei Carabici, suddivisione dei Bembidii, sprovveduto d'occhi, fu da lui (Cav. F. Baudi) rinvenuto per la prima volta in piccole gallerie sotterranee scavate da altri animaletti sotto sassi, ed un alto strato di foglie di faggio nelle foreste di questi alberi delle pendici volte a settentrione della catena dei monti liguri fra il monte Antola ed il Penice. Questi animaletti, vivacissimi nei loro movimenti, appena colpiti dalla luce, cercan rifugio rapidissimamente in qualche fesso del terreno; se non vi riescono, se sono tenuti sulla palma della mano o posti in qualsiasi modo su di una superficie illuminata, fanno due giri convulsamente, si arrovesciano sul dorso, e subito muoiono come fulminati ». 
fra gli Ortotteri; hanno ocelli e occhi composti la maggior parte degli Ortotteri, dei Rincoti, dei Ditteri e qualche genere fra i Coleotteri (Pausus, Ontophagus, ecc.); hanno solamente occhi composti tutti gli altri insetti.

Data cosi un'occhiata rapidissima alla struttura dell'occhio degli insetti, possiamo domandarci come si faccia la visione per mezzo degli occhi composti. Non si può nello stato attuale della scienza rispondere in un modo soddisfacente a questa questione non essendo molto probabilmente giusta la spiegazione data da Giovanni Müller, il quale crede che gli insetti per mezzo degli occhi composti non possano vedere nettamente gli oggetti; ciascuna faccetta secondo lui non può ricevere che l'impressione di una piccola porzione di un oggetto.

\section{Bibliografia.}

Oltre le opere ripetutamente citate intorno all'anatomia degli insetti (Vedi bibliografie precedenti), si consultino gli scritti seguenti :

F. LEYdig. - Anatom. und Histolog. über die Larve von Corethra plumicornis. Zeit. f. wissen. Zool., 1851, vol. 3.

- Zur Anatomie der Insekten. Müller's Archiv., 1859.

Grimm. - Zur Anat. der Fuhler der Insekten. Mém. de l'Acad. de Saint-Pétersbourg, 1869.

Hickes. - On a new Structure in the antenne of Insectes Trans. of the Lim. Soc., 1859, vol. XXII.

ClaparÉde. - Sur les prétendus organes auditifs des antennes chez les Coleoptères lamellicornes et autres insectes. Ann. Sc. Nat. $4^{\text {a }}$ serie, vol. X, 1858.

LANDoIs. - Das Gehörorgan des Hirschkäfers. Arch. für mikrosk. Anat., vol.VI, 1868.

Marcel de Serres. - De l'odorat et des organes qui paraissent en être le siége chez les Orthoptères. Ann. du Muséum, vol. XVII, 1811.

A. LefÈbure. - Note sur le sentiment olfactif des antennes. Ann. Soc. Ent. de France, vol. VII, 1839. 
Perris. - Sur le siége de l'odorat dans les articulés. Ann. des Sciences Nat., $3^{a}$ serie, vol. XIV, 1850.

Siebold. - Ueber das Stimm und Gehörorgan der Orthopteren. Archiv.f. Natur., vol. I, 1844.

Latreinle. - De l'organe musical des Criquets et des Truxales. Mém. du Mus vol. VIII, 1822.

Hensen. - Ueber das Gehörorgan von Locusta. Zeit. f. wiss.Zool., vol. XVI, 1866.

LESPÈs. - Mém. sur l'appareil auditif des Insectes. Ann. des Sc. Nat., serie $4^{\mathrm{a}}$, vol. IX, 1858.

Clapark̀de. - Sur les prétendus organes auditifs des antennes chez les Coléoptères lamellicornes, ecc. Ann. des Sc. Nat., $4^{\text {a }}$ ser., vol. X, 1858.

Hicks. - On a new. organ in Insects. Proc. of Lim. Soc. Zool., vol. I, 1857.

LEYDIG. - Ueber die Geruchsund Gehörorgane der Krebse und Inscckten. Archiv. f. Anat. und Physiol., 1860.

BERTÈ. - Contribuzione all'anatomia e fisiologia delle antenne degli Afanitteri. Atti della R. Accad. dei Lincei, Roma 1878.

KüxcheL. - Rech. sur l'organisation et le développement des Volucelles. Paris, 1876.

Will. - Beitr. zur Anatomie der zusammengesetzten Augen, 1840. Ueber einen eigentümliche Bewegungs. Apparat in den facitterten Insektenaugen. Müller's Archiv., 1852.

Lexdig. - Zum feineren Bau der Arthropoden. Müller's Archiv. f. Anat., 1855.

- Die Augen der Gliederthiere, ecc., 1864.

Claparède. - Zur morphologie die zusammengesetzten Augen der Krebse und Insekten, 1868.

M. Schultze. - Untersuch. ü. d. zusam. Augen der Krebse und Insekten, 1868.

Grenaker. - Zur Morphologie und Physiologie der facetteren Artropoden. Auges Göttin. Nach., 1874.

- Untersuch. ü. das Arthropoden. Auge. Klinischen Monatsbät., XV, Rostoch, 1877. 


\title{
XI.
}

\section{FUNZIONI DELLA VITA VEGETATIVA}

\author{
NUTRIZIONE.
}

Funzioni della vita vegetativa sono, come tutti sanno, essenzialmente la nutrizione e la riproduzione, le quali comprendono un vario numero di atti o funzioni secondarie. Cominciamo dalla nutrizione, la quale comprende la digestione, la circolazione, la respirazione, l'assimilazione, ecc. Il canal digerente è l'organo principale della digestione. Il canal digerente è negli insetti costituito, come in tutti gli altri artropodi, ed in generale come nella maggior parte degli animali, da un tubo a pareti chiuse avente due aperture alle due estremità opposte che servono l'una per l'introduzione dei materiali alimentari, l'altra per l'uscita delle parti inutili dei materiali stessi.

La presenza delle due aperture del canal digerente e la loro posizione è un carattere di superiorità degli insetti, ed in complesso degli artropodi sopra un grande numero di invertebrati. Nei molluschi l'orifizio anale tende ad avvicinarsi all'orifizio boccale, nei polipi veri si confonde con questo, ed il canal digerente ha una sola apertura; nelle spugne finalmente manca al tutto un canal digerente propriamente detto.

Considerato dal punto di vista istologico, il canal digerente degli insetti è costituito di varie membrane o tuniche sovrapposte.

L'impalcatura delle pareti del canal digerente è formata da sostanza connettiva e si presenta a noi sotto la forma di uno strato omogeneo che prende il nome di tunica propria del canal digerente: internamente la tunica propria o connettiva è ta- 
pezzata da uno strato epiteliale (strato che nella parte posteriore del canal digerente, come ho già avuto occasione di dire, si stacca nelle mute della pelle), esternamente la tunica propria è avvolta da uno strato muscolare più o meno robusto, che presenta delle fibre disposte longitudinalmente e delle fibre disposte circolarmente. Esternamente al tutto poi spesso si nota un sottile strato membranoso distinto col nome di sierosa.

Il canal digerente degli insetti appare istologicamente, da quanto si è detto, in fondo costituito come quello degli animali superiori, infatti: la tunica propria col suo strato epiteliale interno corrisponde allo strato connettivo e all'epitelio dei vertebrati, lo strato muscolare corrisponde alla tunica muscularis, e finalmente l'ultimo straterello più esterno è paragonabile alla sierosa.

Le parti or ora menzionate non si trovano naturalmente tutte in tutte le parti del canal digerente e con egual grado di sviluppo.

Vediamo anzitutto quali siano le parti principali del canal digerente.

II tubo o canal digerente negli insetti si porta dall'estremità anteriore all'estremità posteriore del corpo, ora seguendo una linea retta, ora invece descrivendo un numero più o meno grande di giri o circonvoluzioni. Certi rigonfiamenti e certi restringimenti che tratto tratto si incontrano lungo il canal digerente ne separano le varie parti.

Procedendo dalla parte superiore troviamo:

$1^{\circ}$ L'apparato boccale,

$2^{\circ}$ L'esofago,

$3^{\circ}$ Lo stomaco,

$4^{\circ}$ L'intestino.

Queste parti principali si suddividono poi e si modificano, come vedremo, molto diversamente nei vari ordini di insetti.

Accompagnano queste parti varie maniere di ghiandole, e soprattutto ghiandole salivari, e un numero più o meno grande di fili o tubi esilissimi che dal nome del loro scopritore vennero detti vasi Malpighiani.

La natura del cibo pare abbia una grande influenza sulla conformazione generale del canal digerente, e, come si osserva spesso nel regno animale, il canal digerente è, paragonato alla 
lunghezza del corpo, molto più lungo negli animali fitofagi che non nei carnivori.

Questa legge soffre nel mondo degli insetti moltissime eccezioni. Nelle modificazioni del canal digerente degli insetti ha maggior importanza il grado di consistenza del cibo. Il canal digerente in generale è più semplice e meno robusto negli insetti che si nutrono di sostanze liquide, più complesso e molto più robusto in quelli che si nutrono di alimenti solidi.

APPARATO BOCCALE.

Prendiamo per maggior chiarezza a considerare l'apparato

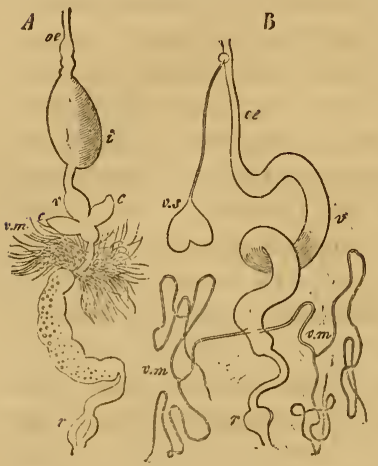

Fig. 33.

$A$ canal digerente di un grillo $-B$ canal digerente di una mosca - oe esofago $i$ ingluvie $-v$ stomuco $-c$ appendici $r$ reito - vm vasi Malpighiani - vs stomaco succiatore. boccale di un insetto masticatore, ad esempio, quello della comune locusta.

L'apparato boccale della locusta (l'apertura della bocca, si noti, è qui, come in tutti gli insetti, disposta secondo un diametro antero-posteriore e non trasversale come nei vertebrati) consta delle parti seguenti:

$1^{\circ}$ Labbro superiore,

$2^{\circ}$ Mandibole,

$3^{\circ}$ Mascelle,

$4^{\circ}$ Labbro inferiore.

Queste parti si trovano nell'apparato boccale di tutti gli insetti, e costituiscono, modificandosi più o meno profondamente, gli apparati succiatori, lambenti, pungenti, ecc.

Il labbro superiore negli insetti masticatori è collocato nella parte anteriore della bocca; è trasversale e presenta quasi sempre traccia della sua primitiva divisione in due parti simmetriche in una sutura antero posteriore.

Al disotto del labbro superiore e lateralmente si trovano le mandibole, le quali sono per lo più molto robuste e dentate nel loro margine interno (1).

(1) Intorno allo sviluppo anormale di queste parti, ed il loro trasformarsi in 
Le mascelle sono collocate sotto alle mandibole, ma un po' più all'indentro di queste. Sono meno robuste, ma hanno denti molto acuti, portano per lo più un paio di palpi e servono a compiere la triturazione degli alimenti cominciata dalle mandibole.

Il labbro inferiore si presenta foggiato come il superiore di due parti simmetriche saldate insieme, e porta nel massimo numero dei casi un paio di palpi (1).

Negli insetti succiatori, ad esempio nei Lepidotteri, le parti della bocca si modificano nel modo seguente:

Il labbro superiore è ridotto ad una sottile lamina trasversale collegata immediatamente al disotto della fronte: le mandibole sono al tutto rudimentali e sono poste secondo il solito inferiormente e lateralmente al labbro superiore. Al disotto delle mandibole atrofizzate abbiamo le mascelle, le quali si allungano straordinariamente e unendosi fra loro formano la proboscide o tromba caratteristica dei Lepidotteri. Una prova di questo fatto l'abbiamo anche nella presenza alla base di ciascuna metà della tromba di un palpo rudimentale. Piccolissimo è il labbro inferiore, relativamente enormi sono invece i palpi labiali.

Nei Rincoti l'apparato boccale è destinato a succiare, e le varie parti che lo compongono sono modificate nel modo seguente:

Il labbro superiore, largo alla base, si prolunga anteriormente, restringendosi verso l'apice, per un quarto circa della lunghezza dell'intiero apparato succiante. Le mandibole o le mascelle si allungano straordinariamente e sono appuntate e dentate alla loro parte inferiore. Il labbro inferiore è molto sviluppato, si prolunga anteriormente e costituisce una proboscide succiante.

organi di difesa, o di offesa, o di ornamento, vedi Cap. IV e V. Le mandibole delle larve dei Ditischi presentano una struttura speciale, esse sono percorse la un canaletto che si apre esternamente nella loro estremità superiore, internamente nell'esofago. Per mezzo di questo canaletto l'animale succhia gli umori dell'animale che ha ucciso.

(1) Per ciò che è delle modificazioni che subiscono le parti sopradette della bocca nelle varie famiglie di insetti, vedi la parte descrittiva. 
Nei Ditteri, in cui l'apparato boccale è pure succiante, il labbro superiore è foggiato come quello dei Rincoti, è più allungato tuttavia e giunge fino all'estremità inferiore delle ma-
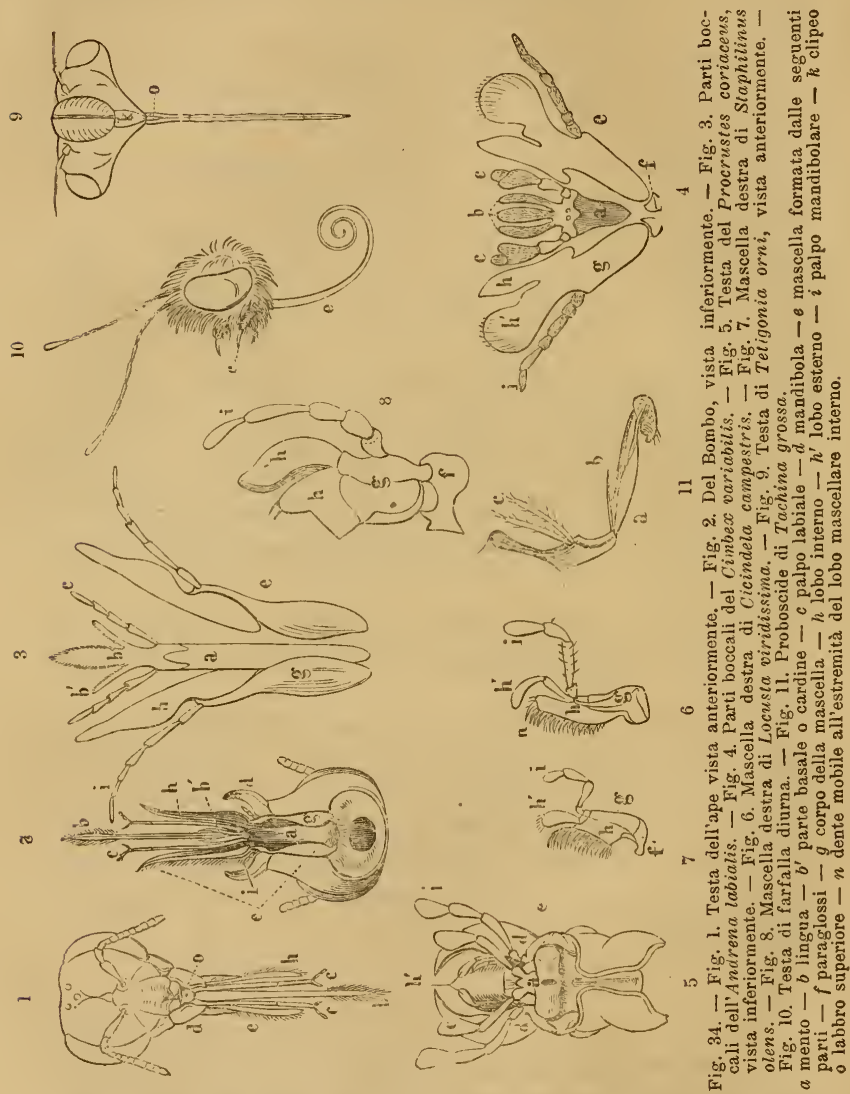

scelle. Le mandibole e le mascelle sono come nei Rincoti. Le mandibole tuttavia spesso non sono dentate all'estremità inferiore. La parte interna o linguetta è allungata analogamente 
alle mandibole. Il labbro inferiore è allungato in una guaina (1) come nei Rincoti.

Negli Imenotteri ora l'apparato boccale è foggiato per masticare, ora per lambire. In questo ultimo caso il labbro superiore è trasversale, le mandibole e le mascelle sono piccole, il labbro inferiore invece è molto sviluppato e trasformato nell'organo lambente.

L'apparato boccale può variare nello stesso insetto nei vari stadi del suo sviluppo. Ciò si può osservare soprattutto nei Lepidotteri in cui l'apparato boccale dei bruchi è atto a masticare, quello invece degli insetti perfetti a succiare.

Le pareti interne della cavità boccale sono per lo più spalmate da un liquido analogo alla saliva degli animali superiori e capace di trasformare le sostanze feculacee in glucosio. Nella cavità boccale si trovano l'epifaringe e l'ipofaringe o lingua. La prima è collocata all'indietro del labbro superiore, la seconda all'indietro del labbro inferiore. L'ipofaringe spesso è molle e carnosa e molto probabilmente, come ho già detto, serve al gusto.

Alla bocca tiene dietro l'esofago, il quale ha la forma di un tubo allungato, ora stretto come negli insetti che si nutrono di sostanze liquide, ora di un diametro relativamente molto grande come in quelli che si nutrono di sostanze solide e soprattutto vegetali.

L'esofago presenta molto sovente un rigonfiamento analogo a quello che si trova in molti uccelli e che viene pure chiamato ingluvie o gozzo. In questo rigonfiamento l'animale fa provvista di cibo che puo in qualche caso, come l'ape, rigurgitare.

In qualche caso l'ingluvie si sviluppa tanto da costituire un vero stomaco, diviso dall'eśofago da un lungo collo che si apre nell'esofago stesso in prossimità della bocca. Questo stomaco piglia il nome di stomaco succiante. Questa dilatazione speciale dell'esofago farebbe, per quanto se ne sa oggi, l'uffizio d'una tromba aspirante. Lo sviluppo e la forma di questa parte del-

(1) Non tutti gli Autori sono d'accordo nello spiegare così la formazione delle parti della bocea dei Rincoti. Le opinioni variano soprattutto per ciò che riguarda la laminetta impari posta al disopra della guaina. 
l'esofago è varia (1), e pare sia in rapporto collo sviluppo della proboscide. Nei Lepidotteri lo stomaco succiante non contiene ordinariamente che aria.

Dopo l'esofago abbiamo lo stomaco, il quale nella maggior parte dei casi si divide in due parti : nello stomaco muscolare che per la sua rassomiglianza con quello degli uccelli piglia il nome di ventriglio, e nello stomaco propriamente detto, o ghiandolare, o anche, come viene chiamato impropriamente, chilifero.

Il ventriglio è destinato a tritare il cibo prima che questo venga a contatto coi sughi destinati a decomporlo. Esso è ben sviluppato negli insetti che si nutrono di sostanze solide vegetali od animali (in tutti gli Ortotteri e nei Carabici, nei Ditiscidi, ecc. fra i Coleotteri), poco sviluppato al contrario, ed anche al tutto mancante negli insetti che si nutrono di sostanze solide poco dure, o di sostanze liquide (Lamellicorni, Coccinelle, fra i Coleotteri e molti Nevrotteri). È rudimentale negli Imenotteri, nei Rincoti, nei Lepidotteri (2) e nei Ditteri.

Il ventriglio negli insetti presenta per lo più una serie di forti colonne muscolari, spesso in numero di sei, armate molte volte di robuste spine o piastre chitinose le quali servono efficacemente alla triturazione degli alimenti.

Dal ventriglio, per mezzo di un'apertura provvista per lo più di uno sfintere e in qualche caso anche di un apparecchio ralvolare detta cardia (e da qualche autore non molto propriamente piloro), si passa nello stomaco chilifico. È in questa parte del canal digerente che negli insetti arviene principalmente la decomposizione chimica degli alimenti, venendo questi in essa a contatto coi sughi gastrici.

Nelle pareti dello stomaco chilifico, noterò subito, si trova una grande quantità di ghiandole che sboccano nell'interno del ventricolo stesso e che hanno una struttura analoga alle ghiandole che producono la pepsina negli animali superiori.

La forma e le dimensioni dello stomaco propriamente detto

(1) Nelle zigene, ad esempio, fra i Lepidotteri è profondamente bilobo.

(2) Nei Lepidotteri allo stato di bruco, si trova in qualche caso un ventriglio ben sviluppato, quantunque privo di parti dure. Cio è in rapporto colla natura del nutrimento dei bruchi stessi. 
variano assai negli insetti. Ora è allungato e sacciforme, ora invece cordiforme, ora presenta la superficie esterna liscia, ora invece coperta di tubercoli e di spine e di piccole ghiandole, ecc., come si osserva nel ditisco ed in altri coleotteri carnivori. 亡̀ più sviluppato tuttavia generalmente negli insetti fitofagi che non nei carnivori, più nello stato larvale che non nello stato di insetto perfetto.

In molti insetti lo stomaco chilifico porta varie appendici o prolungamenti canaliformi terminati a fondo cieco. Queste appendici possono avere la forma di sacche allungate (le quali qualche volta si riempiono di cibo), che pigliano il nome di ciechi gastrici o di borse ventricolari, ovvero di tubi lunghi e sottili a mo' di peli, ed allora prendono il nome di villosità.

Le borse ventricolari sono molto sviluppate, in generale, negli Ortotteri e in molti Coleotteri, ma solo nello stato larvale (1).

Non si sa bene ancora quale sia l'uffizio di queste appendici, non pare tuttavia che esse preparino liquidi destinati alla digestione. Le villosità che si trovano in buon numero di insetti hanno invece, a quanto pare, un uffizio schiettamente ghiandolare.

L'intestino, che tiene dietro allo stomaco propriamente detto, può venir diviso in due parti, nell'intestino tenue e nell'intestino crasso, o colon, o serbatoio stercorale.

In generale il punto di inserzione dei vasi Malpighiani indica il punto di partenza dell'intestino dallo stomaco chilifico. Il Milne-Edwards chiama parte pilorica del canal digerente quella nella quale vanno a sboccare i vasi Malpighiani.

In qualche caso l'intestino tenue è al tutto rudimentale, come si osserva in parecchi Rincoti, in altri è invece molto lungo, sottile e circonvoluto, come ad esempio nell'Oryctes nasicornis.

Il serbatoio stercorale non è altro in fondo che una dilatazione dell'intestino più o meno spiccata secondo le specie e

(1) Qualche specie di Coleotteri presenta anche allo stato di insetto perfetto un certo numero di borse ventricolari: sono in questo caso, l'Elater murinus, i dermesti ed altri pochi. 
contenente organi ghiandolari più o meno ben sviluppati. Talvolta, come si osserva quasi generalmente nei Lepidotteri allo stato d'insetto perfetto, in qualche Coleottero (ditisco, necroforo, ecc.), nelle Nepe e nelle Ranatre fra $\mathrm{i}$ Rincoti, ed in qualche Dittero allo stato larvale, il colon ha la forma d'una sacca che si prolunga lateralmente.

Una disposizione molto importante dell'intestino dev'essere qui accennata. Le larve acquatiche delle libellule e di altri insetti strettamente legati nel primo loro stadio di sviluppo coll'acqua presentano nell'ultima porzione dell'intestino una serie di lamelle ricche di vasi tracheali, lamelle che venendo a contatto dell'acqua che penetra nell'intestino servono come organi respiratori. In altri insetti le lamelle sono ridotte a semplici bitorzoletti ricchi di vasi tracheali, ma che non servono più alla respirazione.

L'ultima porzione del canal digerente paragonabile al retto degli animali superiori si apre esternamente coll'apertura anale. Questa apertura in parecchie specie, secondo certi autori, soprattutto nello stato larvale, mancherebbe intieramente.

Molte ghiandole, come ho già detto, accompagnano il canal digerente degli insetti. Esse possono venire divise essenzialmente in due categorie, in ghiandole salivari ed in vasi urinobiliari o Malpighiani.

Le ghiandole salivari mancano non raramente al tutto in certi gruppi di insetti (sono privi di ghiandole salivari, a quanto pare le Libellule, le Efimere e la maggior dei Coleotteri pentameri), in altri sono appena rudimentali, in altri ancora come negli Ortotteri sono sviluppatissime.

Le ghiandole salivari, che sono collocate, nel massimo numero dai casi, ai lati dell'esofago nella sua parte superiore e si aprono nella cavità boccale, sono, morfologicamente parlando, di due sorta. Vi hanno cioè delle ghiandole a grappolo e delle ghiandole costituite semplicemente da un tubo o sacco allungato (1). Queste due sorta di ghiandole possono trovarsi isolatamente

(1) Non tutti gli Autori sono d'accordo nel considerare come ghiandole salivari queste sacche. Il Milne Edwards li crede semplici serbatoi della saliva. Altri fra cui il Gegenbaur ed il Blanchard li credono, a buon diritto, vere ghiandole salivari. 
nelle varie famiglie di insetti, od anche contemporaneamente nella stessa specie, come si osserva appunto nella comune cavalletta.

E cosa certa che, ad esempio, nella comune cavalletta vi hanno due sorta di ghiandole, i prodotti delle quali vengono a mescolarsi nella cavità boccale. Una cosa non sappiamo ancora rispetto a questi liquidi, ed è se abbiano o no fra loro eguali proprietà chimiche.

Le ghiandole salivari si trasformano negli insetti (lo stesso fatto avviene pure nei vertebrati) molto frequente in ghiandole velenifere. Le ghiandole secernenti la seta, sviluppatissime soprattutto nelle larve di molti Lepidotteri, provengono pure secondo molti autori da ghiandole salivari trasformate.

Grandi incertezze si hanno ancora intorno alla struttura ed intorno all'uffizio dei vasi Malpighiani. Questi organi hanno la forma di tubi allungati e sottili e sono terminati nella massima parte dei casi a fondo cieco. Essi sono impiantati per l'uno dei capi nella parte inferiore dello stomaco chilifico, l'altro capo o è libero o viene, come si osserva in qualche raro caso, ad attaccarsi all'intestino tenue (l) (ciò si osserva in molti Coleotteri e forse anche in qualche Nevrottero).

Il numero dei vasi Malpighiani è molto diverso nei vari ordini degli insetti. Nei Ditteri o nei Rincoti se ne hanno, in gene-

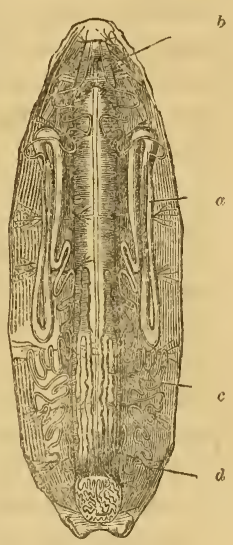

Fig. 35 .

Larva di filugello. a ghiandole secernenti la seta - $b$ luru canale escreture - $c$ canal digerente - $d$ vasi Malpighiani. rale, due paia; nei Lepidotteri, ed in molti Coleotteri ed in molti Nevrotteri tre paia. Gli Ortotteri e gli Imenotteri sono fra tutti gli insetti quelli che ne contano un maggior numero (qualche centinaio).

Mancano intieramente, a quanto pare, in qualche specie di Coccus e negli Aphis fra i Rincoti.

(1) Secondo le ricerche più recenti i vasi Malpighiani che si inseriscono anche sull'intestino tenue, non si aprono in questo. 
In qualche caso sembra vi siano, come nelle ghiandole salivari, due sorta di vasi Malpighiani, come si può osservare facilmente nell'Oryctes nasicornis, ed in altri Coleotteri.

Rispetto alla forma ed alla disposizione dei vasi Malpighiani osserverò ancora che in molti Ditteri i due vasi Malpighiani di ciascun lato si uniscono fra loro e sboccano nel canal digerente per mezzo di un solo condotto. Nei Ditteri si osservano pure talvolta vasi Malpighiani che, o hanno l'estremità libera dilatata a mo' di un'ampolla, o presentano alla loro base una dilatazione che serve di serbatoio del liquido della ghiandola. Ora finalmente i vasi Malpighiani sono lisci, ora verrucosi, ora frangiati come nella comune Melolonta.

I vasi Malpighiani creduti dapprima (Cuvier) semplici vasi biliari, vennero più tardi invece considerati come vasi urinari (Sirodot) (1). Modernamente, il Leydig prima, poi il Fabre ammettono che essi servano ad un tempo come organi biliari e come organi urinari. La questione non è ancora risolta.

Quello che è certo si è che i vasi Malpighiani servono alla eliminazione dei materiali diventati inutili nel sangue.

Venne fatto a questo proposito un esperimento semplicissimo. Mescolando al nutrimento di un bruco una qualche materia colorante, dell'indaco ad esempio, si vede che in poco tempo il sangue diviene fortemente colorato. Cessando di dare al bruco della materia colorante, il sangue si scolora poco a poco e la materia colorante si accumula invece nei vasi Malpighiani.

Prima di lasciare l'argomento degli organi ghiandolari che accompagnano il canal digerente, menzioniamo ancora i corpi adiposi e le ghiandole anali.

Negli insetti i corpi adiposi sono in generale molto sviluppati, e a quanto pare non sarebbero solo depositi di grasso, ma conterrebbero eziandio delle secrezioni speciali, ad esempio dei sali urici.

(1) L'analisi chimica del liquido contenuto in questi tubi, fatta da prima dallo Chevreul e ripetuta poi da altri, dimostrò la presenza in essi dell'acido urico. 
In qualche caso, Lampiridi, ecc., i corpi adiposi costituiscono gli organi fosforescenti di cui ho già parlato.

Numerosissime sono le ghiandole che sono collocate in prossimità dell'ultima parte del canal digerente e che versano il loro prodotto o nell'ultima porzione dell'intestino o direttamente allo esterno. A questa categoria di organi appartengono gli apparati esplodenti dei Brachinus e di altri Coleotteri, e gli apparati produttori dei liquidi puzzolenti ed acri che molti insetti schizzano fuori dall'ano, come è stato detto nel capitolo IV.

Veduto cosi quali siano le parti essenziali che costituiscono il canal digerente degli insetti, esaminiamo brevemente come le tre tuniche, che formano le pareti del canal digerente stesso, si modifichino nelle varie sue parti.

Lo strato interno epiteliale per lo più manca nell'esofago e secondo il Mekel anche nello stomaco succiante degli Imenotteri e dei Ditteri; manca anche, come ha osservato il Leydig, nel retto della larva del formicaleone. Negli insetti, almeno per quanto si sa oggi, l'epitelio dell'intestino non è mai vibratile.

La tunica propria si presenta ora poco sviluppata e molle, ora invece molto grossa e chitinizzata, come si osserva principalmente nel ventriglio di parecchie specie: lo strato più esterno ha spesso la consistenza del tessuto chitinoso ed anzi presenta molto evidenti i pori canali, di più, in molti casi, ha delle ripiegature e degli infossamenti che ricordano la forma cellulare e più particolarmente i corpuscoli ossei. Non si tratta qui tuttavia di vere cellule, ma di semplici ripiegature del tessuto chitinizzato.

La tunica muscolare spesso manca nell'intestino, quando esiste, parte delle sue fibre sono disposte longitudinalmente e parte circolarmente.

Le fibre muscolari, a quanto pare, sono sempre striate ed inoltre non infrequentemente si ramificano fra loro.

Il cibo introdotto nella bocca viene anzitutto bagnato dalla saliva la quale puó essere limpida e inodora, ovvero gialliccia o verdiccia e di un odore acre, ed avere un'azione corrodente molto spiccata.

Pare che la saliva degli insetti abbia reazione alcalina e 
come quella dei vertebrati eserciti una prima azione chimica sugli alimenti (1).

Nello stomaco il cibo viene a contatto del sugo gastrico, il quale anche negli insetti, come nei vertebrati, ha sempre durante la digestione una reazione acida, la quale non diviene alcalina che quando l'animale rimane per qualche tempo digiuno. Nell'intestino poi il cibo è sottoposto all'azione delle numerose ghiandole che, come abbiamo visto, versano il loro prodotto in questa parte del canal digerente.

Negli insetti la chimificazione e la chilificazione, dirò in ultimo, non pare siano nettamente separate fra loro (2).

La parte utile del cibo dopo che questo ha subito nel canal digerente le varie modificazioni chimiche nelle quali consiste propriamente la funzione della digestione, passa attraverso alle pareti del canal digerente stesso e viene portata dal sangue per tutte le parti del corpo. Il sangue è negli insetti, come in tutti gli altri animali, il veicolo per mezzo del quale i vari organi possono ricevere i materiali nutrizi e nello stesso tempo possono spogliarsi dei prodotti di ossidazione formatisi in essi in seguito al loro funzionare (3).

Il sangue degli insetti è, come negli animali superiori, costituito da due parti, da una parte liquida é da corpicciuoli solidi nuotanti in essa. Per lo più è incolore, qualche volta tuttavia è leggermente colorato in giallo od in verde. Pare che queste colorazioni dipendano dalla natura del nutrimento e variano col variare del nutrimento stesso; cosi ad esempio il sangue dei bruchi che si nutrono di foglie, riceve dalla clorofilla una

(1) La saliva di molti insetti agisce energicamente sui tessuti vegetali, ed è causa principale della produzione delle galle.

(2) Intorno ai fenomeni della digestione negli insetti, ed in generale negli Artropodi, si consultino i numerosi lavori del Plateau inseriti nel Bulletins de l Académie Royale de Belgique di questi ultimi anni. Non si dimentichi pure un lavoro del signor G. Tursini intorno all'assorbimento intestinale degli Artropodi. Rendiconto dell'Accademia delle Scienze Fisiche e Matematiche di Napoli, anno XVI, 1877.

(3) La circolazione del sangue venne per molto tempo negata agli insetti da insigni naturalisti, fra i quali il Cuvier, e il Leon Dufour. Quest'ultimo anzi sostenne sempre la mancanza di una circolazione negli insetti, e in un lavoro stampato nel 1833 intorno all'anatomia ed alla fisiologia degli Emitteri (Recherches 
colorazione verdiccia, colorazione che non si osserva più nella farfalla. Si possono pure, come ho giá fatto osservare, dare artificialmente al sangue varie colorazioni.

I corpuscoli del sangue degli insetti sono incolori, allungati, fusiformi ed un po' appiattiti. Si trova anche un più o meno grande numero di corpuscoli circolari.

Intorno al numero dei corpuscoli del sangue negli insetti nor si hanno cognizioni precise. In qualche caso (larve di Ditteri) il loro numero è picciolissimo.

Generalmente i corpuscoli negli insetti allo stato larvale sono sprovvisti di nucleo e di granulazioni. Queste parti sono invece molto evidenti negli insetti giunti allo stato perfetto.

Il sangue degli insetti contiene molto probabilmente nel suo siero delle sostanze analoghe alla albumina ed alla fibrina. Esso si coagula infatti se viene esposto all'aria o al calore. Contiene pure vari sali, imperocchè facendone evaporare una goccia su di un pezzo di vetro si ottengono delle cristallizzazioni dendritiche.

Negli insetti, come negli altri animali, l'organo principale della circolazione è il cuore.

Il cuore degli insetti ha la forma di tubo, è allungato e leggermente fusiforme, ed occupa la parte longitudinale e mediana del dorso. Questo tubo o canale dorsale consta essenzialmente di due parti: di una parte anteriore più stretta e che non si contrae, detta aortica, e di una parte posteriore più grossa e che si contrae ritmicamente, detta cardiaca. Questa seconda parte del canal dorsale è la più importante, imperocchè è

anat. e physiol. sur les Hémiptères. Mém. des Savants étrang., vol. IV), ha in proposito le parole seguenti: - "C'est un fait établi, je crois, en principe, que dans les animaux où il y a une circulation générale d'air, celle-ci remplace ou exclut la circulation générale du sang ou d'un liquide analogue. Ces deux systèmes circulatoires sont incompatibles ». Queste idee intorno alla circolazione egli le espone pure in un lavoro sull'anatomia e fisiologia della mosea comune, stampato nel 1845.

La scoperta della circolazione del sangue negli insetti è dovuta al Carus; dopo di lui Wagner, Ehrenberg, Bowerbank, Tyrrell, Dugès, Verloren, ecc., hanno studiato diligentemente il fenomeno, ed oggidi la circolazione del sangue negli insetti è ammessa da tutti. 
quella che contraendosi spinge il sangue nella porzione aortica dalla quale poi passa nelle altre parti dell'organismo.

La porzione cardiaca ha una struttura alquanto complessa. Essa presenta una serie di strozzamenti esterni corrispondenti internamente a una serie di concamerazioni che hanno movimenti di sistole e di diastole. Il numero delle concamerazioni è vario: ve ne hanno sette od otto nei Lepidotteri, nei Coleotteri e negli Ortotteri, quattro o cinque in molti Imenotteri e in molti Ditteri, meno ancora nella maggior parte dei Rincoti. Queste concamerazioni possono essere considerate come una serie di cuori corrispondenti ciascuno ad un zoonito. Ai lati le concamerazioni del cuore portano un paio di orifizi ed un apparato valvolare che concede l'entrata del sangue proveniente dalle varie parti del cuore, ma impedisce che nella contrazione delle pareti del corpo il sangue possa rifluire da questa parte.

La porzione cardiaca inoltre del vaso dorsale è avvolta da una membrana pericardica delicatissima, la quale forma ai lati del vaso dorsale il seno pericardico (paragonabile abbastanza giustamente all'orecchietta del cuore), nel quale viene ad accumularsi il sangue che ha percorso le varie parti del corpo e dal quale il sangue rientra, come ho giả detto, per mezzo degli orifizi laterali delle concamerazioni nel cuore.

La parte cardiaca occupa a un dipresso la lunghezza dell'addome ed è tenuta ferma contro gli archi superiori dei segmenti addominali dalle ali del cuore, espansioni membranose, contenenti una quantità variabile di fibre muscolari, per lo più in numero di sette paia.

Le pareti del cuore sono robuste e costituite da due membrane, l'esterna contiene le fibre muscolari. Sopra a questa vi sono talvolta dei fasci di fibre muscolari che accrescono la robustezza delle pareti del cuore stesso.

La porzione aortica del vaso dorsale si differenzia, come ho già detto, dalla parte cardiaca per la sua struttura. Essa è formata da un semplice tubo membranoso ed è priva delle concamerazioni, degli orifizi laterali e delle ali del cuore. Prende origine generalmente dalla parte cardiaca al principio del torace. Ora essa si incurva in basso per seguire la forma del corpo, come si osserva negli Imenotteri, nei Ditteri, ecc. e in 
tutti gli insetti in cui l'addome è unito al torace con peduncolo, ora invece procede diritta come in molti Ortotteri (soprattutto nei generi Phasma, Bacillus, ecc.). Giunta la porzione aortica nel capo, si caccia sotto i gangli cerebroidi avvicinandosi all'esofago e termina ora bruscamente, ora con piccole e scarse ramificazioni, lasciando uscire il sangue spinto dal cuore. Il sangue che esce dal tratto terminale della porzione aortica del canal dorsale, si distribuisce nelle varie parti del corpo, dalle quali poi, come abbiamo già veduto, ritorna per mezzo del seno pericardico al cuore. Intorno al modo di comportarsi del sangue coi vari organi e soprattutto colle trachee, molto disparate sono tuttora le opinioni dei naturalisti. Sarebbe troppo lungo tuttavia il riferire tutte le ipotesi state escogitate in proposito. Si consultino a questo riguardo le opere citate nella bibliografia alla fine del capitolo. Io mi limiterò ad accennare le spiegazioni date dal Milne Edwards e dal Blanchard, come quelle che molto probabilmente più si accostano al vero. Il Milne Edwards ammette negli insetti una circolazione semilacunare o semivascolare. Il sangue uscito dall'aorta penetra fra vari organi infiltrandosi attraverso ai meati dei loro tessuti e venendo a contatto coll'aria, che vi arriva portata dalle più sottili ramificazioni tracheali, prende l'ossigeno che gli è necessario e si spoglia dei materiali inutili prodotti dalla combustione. Dai vari tessuti ritorna al cuore nel modo che è già stato detto. Secondo il Milne Edwards, la parte vascolare della circolazione sarebbe negli insetti ridotta alla porzione aortica del canal dorsale.

Il sangue nella sua circolazione lacunare dà origine nella massima parte dei casi a correnti abbastanza evidenti. Le principali sono tre, una collocata inferiormente al disotto della catena gangliare (la direzione del sangue è dalla parte anteriore del corpo alla parte posteriore) e due poste ai lati del corpo.

Il Blanchard ammette pure che la circolazione degli insetti sia semivascolare. Egli crede tuttavia che una buona parte del sangue uscito dall'aorta si infiltri fra le due membrane costituenti le trachee e tenute distaccate fra loro, come vedemmo, da un filo chitinoso avvolto a spira e che correndo lungo la trachea possa compiere il necessario ricambio di materiali gazosi coll'aria che entra nel canale tracheale. Giunto 
alle ultime ramificazioni delle trachee, il sangue circolerebbe fra gli interstizii dei tessuti e giungerebbe poi al cuore. Cosi, secondo il Blanchard, il sangue arterioso uscito dalle trachee si mescolerebbe col sangue venoso degli spazi lacunari ed il cuore caccierebbe per l'aorta un sangue misto.

L'ipotesi del Blanchard non è ammessa da tutti, ed il Gratiolet, il Soly ed il Weismann hanno sollevato contro ad essa varie obiezioni. Recentemente tuttavia il Künckel è venuto con nuove ricerche a confermare i fatti esposti dal Blanchard.

Strettamente legata colla circolazione è la funzione della respirazione. Gli insetti nello stato perfetto respirano l'aria atmosferica libera, nello stato di larva la maggior parte respira come gli adulti, gli altri respirano invece l'aria sciolta nell'acqua.

La respirazione si fa negli insetti essenzialmente per mezzo di tubi cilindroidi, detti trachee, che portano l'aria in tutte le parti del corpo.

Le pareti delle trachee sono costituite di un vario numero di membrane o tuniche sovrapposte. La membrana interna è di natura epidermica e può essere considerata come la continuazione dello strato esterno della pelle. Porta infatti talvolta, come l'integumento esterno, dei peluzzi (qualche specie di Coleotteri delle famiglie seguenti: Crisomelini, Longicorni, Elateridi, ecc.), ed inoltre, come è già stato detto, si stacca col rimanente dello strato esterno epidermico nell'epoca delle mute.

La tunica mediana od elastica è pure di natura epidermica (il Dujardin crede anzi che non sia altro che un ispessimento della membrana interna) ed è costituita da un filo chitinoso sottilissimo avvolto a spira.

La tunica esterna è formata da un tessuto molle ed omogeneo. Una quarta membrana si osserverebbe pure, secondo l'Hermann ed il Meyer, esternamente al tutto nei rami tracheali più grossi.

Sezionando un insetto sotto l'acqua, i rami tracheali si distinguono facilmente pel loro aspetto bianco argenteo, dovuto all'aria che è in essi contenuta.

Le trachee si aprono esternamente per mezzo di fori o boccuccie che pigliano il nome di stimme.

Le stimme sono collocate simmetricamente sui lati del corpo. 
Nella regione addominale si aprono fra l'arco superiore e l'arco inferiore: nella regione toracica fra gli anelli o sugli anelli stessi. Il numero delle stimme varia. Se ne contano dieci paia negli Ortotteri; il numero ordinario tuttavia è di nove, di cui otto sono portate dall'addome ed uno dal torace.

Il numero delle stimme è di otto paia, in vari Rincoti (Capse e Miris), in alcuni Pseudonevrotteri (Termiti), e in alcuni Ditteri (Chironomus): è di sette paia nella maggior parte dei Rincoti, in molti Imenotteri, Nevrotteri e Ditteri : di sei, in qualche Dittero (Hippobosca ed Ornithomyia). In qualche Emittero (Aspediotus, Lecanium, Aleurodes, ecc.), le stimme sono in numero di due sole paia. Le larve di molti Ditteri (Sarcophaga, ecc.) sono pure nello stesso caso.

Un solo paio di stimme finalmente osserviamo in vari generi di Rincoti acquaiuoli (Nepa Ranatra), nelle larve di varie Tipule e anche nelle Nicteribie.

Le stimme ora sono semplici fessure (stimme toraciche dei Coleotteri carabici e dei Rincoti), ovvero presentano un cercine detto peritrema, il quale ora è semplice, ora rivestito di peli, ora presenta una membranella con ciglia, ecc.

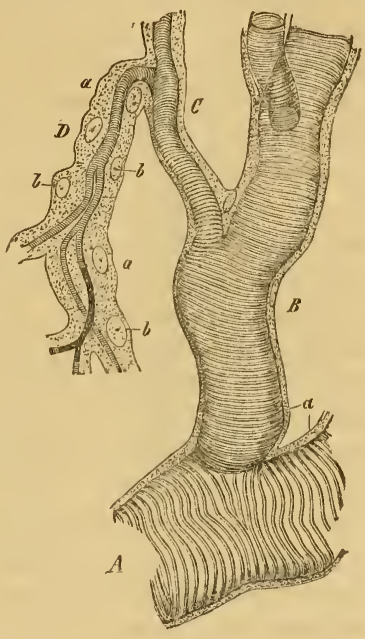

Fig. 36 .

Ramificazioni tracheali $B-C-D$ sirato cellulare epiteliale. $b$ nuclei.

Negli Ortotteri vi hanno due pezzi rigidi che avvicinandosi fra loro, per mezzo di fasci muscolari appositi, chiudono intieramente l'orifizio stimmatico (1).

(1) Ricorderò qui un fatto conosciuto già da lungo tempo e noto a tutti i raccoglitori d'insetti. Introducendo un insetto in un vaso contenente una grande quantità di gaz o di vapori deleteri, come acido solfidrico, ossido di carbonio, 
Ritornando alle trachee osserviamo che avuto riguardo alla loro forma si possono dividere in due grandi categorie: in trachee tubolose e in trachee vescicolose.

Le trachee tubolose sono quelle di cui si è detto poco fa. Le trachee vescicolose hanno una struttura un po' più semplice delle prime, e risultano costituite essenzialmente da due sole membrane: manca o è poco sviluppato il filo spirale. Le trachee vescicolose presentano tratto tratto un numero più o meno grande di piccole cavità, che talora alla loro volta dànno origine ad altre vescicole o cavità più piccole in modo che si ha qui una struttura che ricorda quelle di molte ghiandole ad acino. La comune melolonta offre un esempio bellissimo di questa specie di trachee. Spesso le cavità che partono dalle trachee diminuiscono di numero, ma crescono invece di mole, e pigliano allora il nome di sacche aeree. Queste, si noti, non si trovano che negli insetti giunti allo stato perfetto $\mathrm{e}$ sono intimamente legate al volo.

Secondo le osservazioni del Newport (1) nelle Vanesse, ad esempio, fra i Lepidotteri, le sacche aeree cominciano a formarsi quando l'insetto passa dallo stato di larva a quello di crisalide e si sviluppano poi intieramente quando dalla crisalide passa allo stato perfetto. Il modo di formazione delle sacche aeree è analogo a quello degli aneurismi degli animali superiori.

Ho detto che le vescicole, le trachee e le sacche aeree sono in rapporto col volo, inquantochè, come facilmente si comprende, servono non solo ad accrescere l'attività della respirazione, ma anche a dare maggior leggerezza all'insetto.

benzina, solfuro di carbonio, ecc., si vede che esso, contrariamente a quanto si potrebbe supporre, dura per lungo tempo in vita, e ciò perchè chiude le stimme, ed impedisce al gaz di penetrare nell'interno del corpo. Se invece il gaz contenuto nel vaso è in piccola quantità, l'insetto spesso non si accorge della sua presenza e lasciandolo penetrare nell'interno delle trachee concede al gaz stesso di agire sui tessuti e quindi perisce in un tempo molto più breve. Nello stesso modo si spiega la resistenza vitale degli insetti a dermascheletro iṇolto duro (Coleotteri) immersi nell'alcool od in altri liquidi. Si consulti a questo proposito Michele Lessona: "Sulla resistenza vitale delle mosche nel vino », Atti della R. Acc. delle Sc. di Torino, vol. V, 1869.

(1) « On the respiration of Insects ». Philos. Trans., 1836. 
Esaminando infatti queste parti, si vede che esse sono principalmente sviluppate in quegli insetti che sono molto volatori e che hanno un corpo grosso e pesante.

Negli Ortotteri corridori, blatte, ecc., nelle Mantidi, ecc., le trachee sono tubolose; sono invece spiccatamente vescicolose negli Acrididi. Le vescicole e le sacche aeree sono pure sviluppatissime nei Ditteri, negli Imenotteri e nei Lepidotteri, mancano o-sono poco spiccate nei Nevrotteri e nei Rincoti (nelle cicale le sacche aeree sono ben sviluppate e concorrono alla
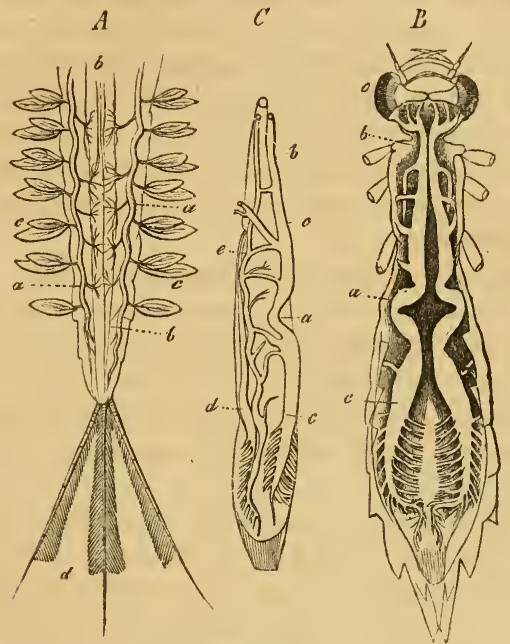

Fig. 37.

A Parte posteriore del corpo della Ephemera vulyata - a tronco tracheale longitudinale $-b$ canal intestinale $-c$ branchie tracheali $-d$ appendici addominali.

$B$ Larva dell'Aeschna grandis vista superiormente - a tronchi tracheali longitudinali superiori $-b$ loro estrémità anteriore $-c$ loro parte posteriore che si raunifica sul retto - o occhi.

$C$ Intestino visto di profilo della larva dell'Aeschna grandis - $d$ tronco tracheale laterale inferiore - e rami tracheali che mettono in comunicazione il tronco tracheale precedente col tronco tracheale superiore: le altre lettere banno lo stesso valore che nella figura $B$.

produzione del suono). Nei Coleotteri mancano quasi sempre, meno che nei Lamellicorni, nei Bupresti e nei Ditisci.

Le trachee, sia tubolose, sia vescicolari, pigliano origine dalle aperture stimmatiche per mezzo di un grosso ramo che viene 
detto trachea di origine, dal quale poi partono più o meno ramificandosi i rami che vanno ai vari organi e che pigliano il nome di trachee di distribuzione.

I vari rami o gruppi tracheali che partono da ciascuna stimma ora sono isolati fra loro, ora invece, come si usserva nella maggioranza dei casi, sono in comunicazione gli uni cogli altri per mezzo di canali anastomotici, detti trachee di comunicazione. I rami tracheali di comunicazione più importanti sono quelli che riuniscono fra loro le trachee d'origine d'una stessa parte del corpo, costituendo un lungo ramo tracheale longitudinale e quelli che riuniscono procedendo trasversalmente le due metà longitudinali dell'apparato respiratorio. Altri rami anastomotici (anastomosi accessorie) vengono in qualche caso ad aggiungersi ai già citati.

Prima di lasciare l'argomento della respirazione degli insetti, diamo un rapido sguardo agli apparati respiratori degli insetti acquaiuoli.

Numerosi sono gli insetti che vivono nell'acqua, ma quasi tutti vengono alla superficie a respirare l'aria atmosferica libera e in essi l'apparato respiratorio non è gran fatto diverso da quello degli altri insetti.

Negli insetti che respirano l'aria sciolta nell'acqua, l'apparato tracheale si trasforma più o meno compiutamente in un apparato branchiale.

Quest'ultima maniera di respirazione si osserva quasi esclusivamente nello stato larrale.

L'apparato branchiale varia assai e nella sua forma e nella sua posizione sul corpo degli insetti.

Ora è l'addome che, come nelle larve delle Efimere, porta due serie longitudinali di lamine membranose dette espansioni branchiali, le quali possono essere in qualche caso ridotte a semplici filamenti, come nel genere Sialis (Nevrotteri), in cui vengono a mettere capo numerose ramificazioni tracheali.

Ora invece le trachee branchiali si trovano collocate, come per primo fece vedere il Réaumur, nelle cavità del retto (larve di libellule) e vengono bagnate dall'acqua, che l'animale immette ed emette ritmicamente dall'apertura anale (1).

(1) Si osservano branchie in qualche insetto adulto come il Newport ha dimostrato pel Pteronacris regalis. 
La respirazione puó essere più o meno attiva secondo le specie e soprattutto secondo le varie condizioni fisiologiche degli individui. Non si hanno, del resto, intorno a questo argomento ricerche sufficienti. In generale pare che i movimenti respiratori siano in media da trenta a trentacinque al minuto.

Una delle conseguenze della circolazione e della respirazione è il calore animale.

Il fatto che gli insetti producono calore era già conosciuto da lungo tempo, ma non si avevano intorno a tal fenomeno cognizioni precise.

Il Réaumur e l'Huber, ad esempio, avevano osservato una forte elevazione di temperatura negli alveari; il Newport anzi aveva constatato che in un alveare in cui le api erano state fortemente eccitate, la temperatura superava di $38^{\circ}$ la temperatura esterna.

L'Haussmann, tuttavia, fu il primo che nel 1803 fece esperienze rigorose-sul calore animale degli insetti. Questo argomento venne poi ristudiato dal Davy, dal Newport, dal Nobili e dal Melloni, ed in questi ultimi tempi principalmente dal Girard, alle opere del quale io rimando il lettore vago di maggiori particolari in proposito. Io mi limiterò a riferire qui le principali e più sicure conclusioni a cui si giunse fino ad oggi intorno al calore animale degli insetti:

$1^{\circ}$ Il calore proprio delle larve e delle ninfe negli insetti dalla metamorfosi compiuta è inferiore a quello degli insetti perfetti.

$2^{\circ}$ Lo stato di riposo, il digiuno, il sonno, ecc. producono una diminuzione nel calore animale.

$3^{\circ}$ In generale, tutti gli insetti camminatori hanno una temperatura inferiore a quella degli insetti volatori. Il calore animale è quindi in complesso maggiore nei Lepidotteri, nei Ditteri, negli Imenotteri che non negli insetti degli altri ordini.

$4^{\circ}$ Negli insetti dalla locomozione aerea il calore si concentra nel torace formando un focolare d'intensità proporzionale alla potenza del volo.

$5^{\circ}$ La temperatura propria degli insetti non si abbassa mai al disotto della temperatura del luogo in cui l'insetto si trova.

In generale gli insetti possono resistere a temperature relativamente molto elevate ed a temperature relativamente molto 
basse. Si trovano insetti infatti nelle acque sgorganti dalle sorgenti termali e nelle acque freddissime dei laghi delle alte regioni alpine, sulle arene dei deserti equatoriali e sulle nevi delle Alpi e delle regioni circumpolari (1).

\section{Bibliografia.}

Oltre le opere già ripetutamente citate intorno all'anatomia degli insetti, si consultino pure gli scritti seguenti :

MaRCEL DE SERRES. - Observations sur les Insectes considérés comme ruminants et sur les fonctions des diverses parties du tube intestinal, ecc. Annales $d u$ Muséum, vol. XX, 1813.

L. Durour. - Recherches anat. sur les Carabiques, ecc. Annales des Sc. Nat. (varie inemorie inserite nei volumi degli anni 1824-25-26).

-- Recherches anat. e phys. sur les Hémiptères. Mém. de t Acad. des Sciences Sav. étrang., vol. IV, 1833.

- Recherches anat. e phys. sur les Orthoptères, les Hyménoptères e le Névroptères. Mém. de l'Acad. des Sc. Sav. étrang., vol. VII, 1841.

- Études anatomiques sur une Mouche. Mém. de l'Acad. des Sciences Sav. étrang., vol. IX, 1848.

- Recherches anatom. e phys. sur les diptères. Mém. de l'Acad. des Sciences Sav. étrang., vol. XI, 1851.

Cornalia. - Monografia del Bombice del gelso, 1856.

Sіворот. - Recherches sur les sécrétions chez les Insectes. Ann. des Sc. Nat., $4^{\mathrm{a}}$ serie, vol. X, 1858.

RAмDoHr. - Abhaud. über die Verdauungswer kzeuge der Insecten. Halle 1811. BurMeister. - Anatomical observations upon the Larva of Calosoma sycophanta. Trans of the Ent. Soc. of London, vol. I, 1836.

L. Dofocr. - Mém. sur les vaisseaux biliaires ou le foie des Insectes. Ann. de Sc. Nat., $2^{\text {a }}$ serie, vol. XIX.

(1) I Ditteri, soprattutto i Chironomus, sono forse fra tutti gli insetti quelli che possono resistere a temperature più basse. Anche fra noi si vedono svolazzare talvolta in grande quantità intorno ai cespugli nelle più rigide giornate d'inverno. 
H. Müller. - Micrographie einiger drüsenapparate der niederen Thiere. Müller's Archiv. f. Anat. und Phys., 1846.

Carus. - Entdeckung eines einfachen vom Herzen ans beschleunigten Bluthreislaufes in den Larven netz flüglicher Insecten. 1827, Leipzig.

Verloren. - Mém. sur la circulation dans les Insectes. Mém. de l Acad. de Bruxelles. Sriv. étrang., vol. XIX, 1844.

L. Dufour. - Recherches anat. e phys. sur les larves des Libellules. Ann. des Sc. Nat., $3^{\text {a }}$ serie, vol. XVII.

S. Kunckel. - De l'existence des vaisseaux capillaires artériels chez les Insectes. Comp. Rend. de l'Acad. des Sciences, 1868.

A. Weismans. - Die Entwickelung der dipteren. Zeitsch. fur wiss. Zoolog., volume XIII e XIV.

- Die Metamorphose der Corethra plumicornis Leit. f. wiss. Zool., vol. XVI, 1860.

DE Filıppi. - Alcune osservazioni anatomico-fisiologiche sugli insetti in generale e in particolare sul Bombice del Gelso. Ann. della R. Acc. d'Agricolt. Torino, 1852, vol. V. 


\title{
XII.
}

\section{FUNZIONI DELLA VITA VEGETATIVA}

\author{
RIPRODUZIONE.
}

Negli insetti la riproduzione è sempre sessuale, i sessi sono sempre portati da due individui distinti. I casi di ermafrodismo sono casi anormali, relativamente molto rari, e per quanto io so non sono mai stati osservati individui ermafroditi che abbiano dato opera alla riproduzione (1). Gli organi della riproduzione maschili e femminili offrono fra loro, in tutti gli insetti, come negli animali superiori, una perfetta corrispondenza di parti e sono collocati nella porzione ventrale dell'addome verso gli ultimi segmenti. Essi sono inoltre, cosa molto importante, formati da parti simmetriche.

Le aperture esterne degli organi della riproduzione vanno ad aprirsi un po' all'indietro dell'apertura anale.

(1) Vennero osservati casi relativamente frequenti di ginandromorfismo nelle api. I Lepidotteri offrono talvolta easi di ermafrodismo nelle ali ; non si hanno dati tuttavia per affermare se l'ermafrodismo si estende o no anche agli organi essenziali della generazione: "Non è, dice a questo proposito il Ghiliani (Ermafroditismo e dimorfismo riuniti in un medesimo Lepidottero. Bulletin Ent. Ital., IX, 1877), che sieno molto rare in natura queste anamolie, massime tra i Lepidotteri, ma passano inosservate e solo colpiscono lo sguardo del raceoglitore, allorquando eapitano in quelle specie $\mathrm{i}$ cui sessi sono assai diversi tra loro per la forma o nel colorito: dal che ne risulta nn'asimmetria che rende molto apparente l'individuo abnorme. Bisognerebbe inoltre che un tale individuo eapitasse vivo nelle mani dell'anatonico; poichè sui corpi secchi riescono troppo difficoltose e spesso impossibili le osservazioni intorno agli organi sessuali interni, e non sempre facile lo studio dello stesso apparato copulatore esterno. Aggiungasi pur anehe la ripugnanza del possessore di un tale insetto anomalo a sacrificarlo per le investigazioni anatomiche senza la certezza di un felice risultato scientifico». 
L'agente essenziale della riproduzione è negli insetti maschi, come del resto in tutti gli animali a riproduzione sessuata, lo spermatozoide. Le uova sono il prodotto delle femmine. L'apparato riproduttore maschile e l'apparato riproduttore femminile sono quindi in fondo niente altro che due apparati ghiandolari. Quello del maschio prepara lo spermatozoide, quello della femmina le uova.

Questi apparati ghiandolari, diciamo subito, non hanno mai negli insetti la struttura di una ghiandola semplice, ma presentano per lo più delle più o meno grandi complicazioni di

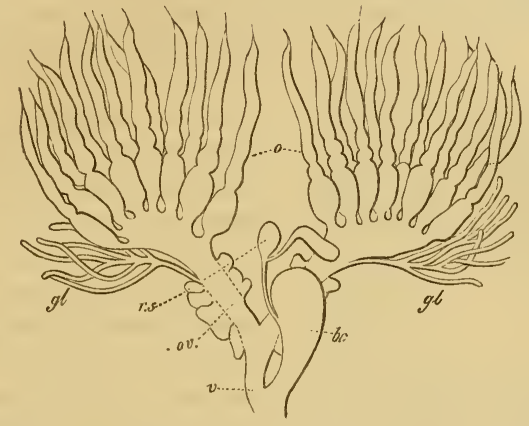

Fig. 38. Apparato riproduttore femminile dell'Hydrobius fuscipes.

0 tubi ovarici $-o v$ ovidotti con appendici ghiandolari $-g l$ ghiandole $-v$ vagina bc borsa copulatrice - rs ricettacolo seminale.

struttura destinate ad assicurare lo sviluppo della prole nelle condizioni più diverse della vita.

L'apparato riproduttore femminile risulta negli insetti costituito essenzialmente dalle parti seguenti: $1^{\circ}$ due ovaie provviste ciascuna di un tubo escretore che si riuniscono fra loro in un canale solo; $2^{\circ}$ dilatazioni di varia natura: borsa copulatrice, ricettacolo seminale, ecc. ; $3^{\circ}$ ghiandole accessorie; $4^{\circ}$ vagina $5^{\circ}$ organi destinati a condurre fuori e a collocare in luoghi convenienti le uova: ovopositori e appendici varie degli ultimi segmenti addominali.

L'apparato riproduttore maschile ha le seguenti parti principali : $1^{\circ}$ due testicoli provvisti ciascuno di un condotto defe- 
rente; $2^{\circ}$ vescicole seminali; $3^{\circ}$ ghiandole accessorie; $4^{\circ}$ pene; $5^{\circ}$ appendici varie degli ultimi segmenti addominali destinate a favorire la copula.

Esaminiamo anzitutto la struttura degli spermatozoidi e dell'uovo.

Gli spermatozoidi degli insetti non differiscono gran fatto da quelli degli animali superiori. Sono filiformi, sottili e generalmente molto lunghi, ora rimangono liberi, ora invece si riuniscono in fasci che talvolta si rivestono di uno strato chitinoso e formano ció che si dice uno spermatoforo, vale a dire una capsula ripiena di spermatozoi. Questo fatto ha dato origine, molto probabilmente, ad un errore intorno al modo in cui si

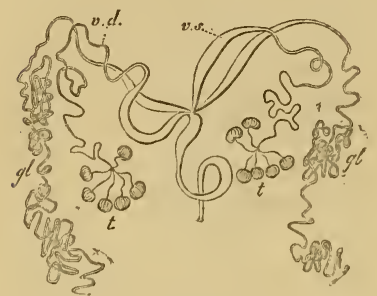

Fig. 39.

Apparato riproduttore maschile della $M e-$ lolontha vulgaris. - $t$ testicoli - vd condotti deferenti - vs dilatazione degli stessi - gl ghiandole. fa la fecondazione in alcuni insetti.

Si era osservato da lungo tempo che dopol'accoppiamento la parte terminale del condotto escretore femminile, la vagina, conteneva un corpo chitinoso allungato che venne creduto il pene del maschio staccatosi dopo la copula. Lo Stein ed altri, studiando meglio la cosa, hanno per molti casi dimostrato che il corpicciuolo in questione non

è altro che uno spermatoforo.

L'uovo degli insetti consta di tutte le parti essenziali che si trovano nell'uovo degli animali superiori, vale a dire, del tuorlo, di una membrana esterna, di una rescicola e di una macchia germinativa; ma queste parti variano moltissimo nella loro struttura.

Sarebbe certamente cosa interessantissima l'esaminare diligentemente tutte le diverse parti dell'uovo e il loro modificarsi in seguito alla fecondazione; ma ciò ci trarrebbe troppo fuori del nostro campo.

Io mi limiterò a menzionare le forme principali e le particolarità più spiccate che si osservano sulle parti più esterne delle uova quando queste vengono deposte. Le uova degli insetti ora hanno forma sferoide, ora invece ovoide più o meno 
allungata, ora vengono deposti isolatamente, ora invece riuniti in gruppi avvolti da sostanze speciali e costituenti le ooteche, ecc. Il guscio esterno delle uova, talvolta durissimo, presentasi ora liscio, ora elegantemente reticolato, in modo da ricordare un tessuto cellulare (1), ora ha numerose granulazioni (Satyrus hyperanthus), ora ha delle costole sporgenti, alla loro volta lisce o granulose (Satyrus tilhonus). La particolaritá più importante che ci presentano le uova degli insetti sono certi forellini piccolissimi detti micropili, pei quali gli spermatozoi possono penetrare nell'interno dell'uovo e dare all'uovo quell'eccitamento speciale pel quale avviene lo sviluppo dell'uovo stesso.

Il Leuckart ha osservato che la disposizione dei micropili varia assai, ed ha caratteri propri nei diversi gruppi d'insetti. Vi ha, ad esempio, un solo micropilo nella massima parte dei Ditteri, ed è collocato al polo anteriore dell'uovo. Nella pulce invece si osservano ai due poli da 40 a 60 forellini. Nei Lepidotteri il numero dei micropili varia da 5 a 20 e sono sempre collocati al polo superiore dell'uovo. In qualche Ortottero i micropili sono collocati invece sulle parti convesse dell'uovo. Nei Coleotteri e negli Imenotteri vi ha in generale un solo micropilo, ed è collocato sul polo anteriore dell'uovo. I micropili finalmente, come si

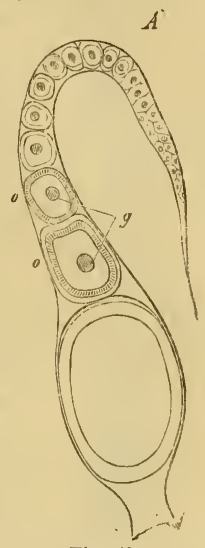

Fig. 40 .

Tubo ovarico della pulce. - o uovo - g vescicula germinativa. osserva in qualche Ortottero ed in qualche Rincoto, presentano una sorta di imbuto.

La parte più importante dell'apparato riproduttore femminile, come abbiamo già detto, è l'ovario.

L'ovario è diviso in due parti simmetriche e simmetricamente collocate nell'addome. Ciascuna di queste due parti ha aspetto piriforme e risulta formata da numero vario di tubi o guaine ovifere, le quali si riuniscono fra loro a costituire un fascio

(1) Non si hanno qui vere cellule, ma solamente le impronte delle cellule epiteliali che tapezzano internamente $\mathrm{i}$ tubi ovarici. 
che superiormente si prolunga in un filamento che si continua col filamento sospensore dell'ovario stesso, ed inferiormente si riuniscono fra loro sopra quella parte dell'ovario che piglia il nome di calice.

Esaminando la struttura di un tubo ovarico, noi vediamo

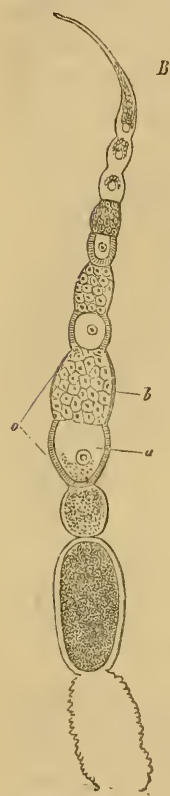

Fig. 41 .

Tubo ovarico di un Carabus violaceus.o scompartimento ovarico divisu in due parti - a cellula dell'uovo $-b$ ammasso vitellogeno. superiormente, nella parte più sottile, una massa cellulare che rappresenta i germi; a mano a mano che dall'apice del tubo discendiamo verso la base, troviamo i germi in gradi successivi di sviluppo e nell'ultima parte del tubo stesso in vicinanza del calice l'uovo quasi al tutto formato. In questo ultimo tratto del tubo ovifero si forma l'involucro esterno dell'uovo con tutte le particolarità di struttura già accennate. Il tubo ovarico si presenta perciò cordoniforme e il suo aspetto varia secondo il grado di sviluppo della uova. Il numero dei tubi ovarici è molto vario: possono essere due soli, uno per ovario, come in qualche Rincoto, o essere in grande numero come nel ditisco fra i Coleotteri. Pare vi sia un certo rapporto fra il numero dei tubi ovarici e il numero delle ampolle spermatiche dei testicoli dei maschi.

Paragonando gli ovari degli insetti con quelli degli animali superiori noi vediamo solo la parte superiore dei tubi oviferi corrispondere veramente agli ovari degli animali superiori, e che il rimanente del tubo stesso puó ritenersi come parte corrispondente agli ovidotti ed all'utero.

Gli ovidotti o canali escretori delle ovaie pigliano origine alla parte inferiore del calice, procedono per un tratto più o meno lungo separati fra loro e poi si riuniscono in un solo canale. Nel loro tratto superiore non presentano nulla di notevole all'infuori di qualche dilatazione in cui in certi insetti le uova soggiornano alquanto. In qualche categoria di insetti gli ovidotti presentano degli apparati speciali che preparano una sostanza glutinosa destinata ad 
avvolgere le uova per proteggerle o per fissarle quando vengono deposte sui vari corpi.

Il canale unico che risulta dalla riunione dei due ovidotti piglia il nome di vagina. Questa presenta pure spesso delle borse e dei rigonfiamenti accessori. Le principali sono il ricettacolo seminale e la borsa copulatrice. Queste parti sono destinate, a quanto pare, a conservare lo sperma, per un tratto di tempo più o meno lungo. Questa disposizione si osserva principalmente sviluppata nell'ape, e pare che la femmina nell'emettere le uova possa lasciare venire o no a contatto delle uova gli spermatozoi contenuti nelle parti sopradette.

Nelle specie finalmente in cui le uova si sviluppano nell'interno del corpo della madre si ha nella parte ultima del condotto comune dell'ovaia una sacca speciale destinata a ricevere le uova e a permettere che le piccole larve si sviluppino.

Questa struttura si osserva spiccata principalmente in molti Ditteri, ad esempio nel genere Echinomyia.

Accompagnano in ultimo le parti essenziali sopradette varie ghiandole, alcune delle quali producono sostanze destinate ad avvolgere le uova quando vengono deposte, altre preparano sostanze di cui si ignora fino ad ora l'uso.

Nei maschi i testicoli sono come gli ovari in numero di due e sono pure collocati simmetricamente (1) nell'addome e risultano dalla riunione di un numero più o meno grande di canali ciechi avvolti sopra se stessi, ora adunati in due grosse masse, come si osserva in vari Ortotteri (ad esempio nell'Acheta campestris), ovvero riuniti in vari gruppi portati da condotti escretori che si raccolgono poi tutti assieme a formare i condotti deferenti, come si osserva in vari Coleotteri (questa disposizione è evidentissima nella comune melolonta). Gli spermatozoi vengono elaborati nell'interno dei ciechi sopradetti.

I testicoli, noterò ancora, sono negli insetti spesso variamente coloriti. La loro tunica esterna è gialla nella Naucoris

(1) In qualche caso i testicoli sono uniti in uno solo. Ciò si osserva non raramente nei Lepidotteri giunti allo stato perfetto. Nei bruchi. sono pure sempre divisi. 
aptera fra i Rincoti, rossa nella Pentatoma dissimilis pure fra i Rincoti, verde invece in molte Sfingi fra i Lepidotteri (1).

I tubi escretori dei testicoli unendosi fra loro danno origine, come ho già detto, ai canali deferenti, i quali unendosi fra loro costituiscono il canal ejaculatore che è più o meno lungo secondo le specie.

Anche qui, come nell'apparato riproduttore femminile, troviamo delle dilatazioni speciali destinate a fare da serbatoio del liquido spermatico e ghiandole, talvolta sviluppatissime, come si osserva nella melolonta, destinate a produrre un liquido speciale di uso non ancora ben noto.

Data cosi un'occhiata rapidissima agli organi essenziali della riproduzione, vediamo come sia foggiato l'apparato copulatore. L'apparato copulatore maschile consta essenzialmente di due parti, vale a dire: di un pene e di un'armatura copulatrice.

Il pene è formato dall'ultima porzione del canale escretore dell'apparato ghiandolare maschile, la quale può rimanere invaginata su se stessa o spiegarsi al difuori. Le sue pareti possono divenire turgide e presentano per lo più delle parti dure chitinose. Talvolta le cose sono un po' più complicate, e vi hanno uno o più involucri esterni formati da altre porzioni del canale escretore stesso disposti in modo da ricordare i vari pezzi di un cannocchiale.

In qualche caso, come ha dimostrato l'Audouin per la pirale della vite (2), vi hanno organi speciali destinati a ritenere il pene nella cavità copulatrice della femmina durante l'accoppiamento. La struttura di queste parti è spesso assai complessa negli insetti. L'armatura copulatrice proviene dalle modificazioni degli ultimi segmenti addominali (3).

Sarebbe cosa troppo lunga e non molto profittevole senza

(1) Intorno alla struttura intima dei canali ciechi costituenti i testicoli, si consulti il trattato più volte citato d'istologia comparata del Leyolig.

(2) «Histoire des Insectes nuisibles à la Vigne et particulièrement de la pyrale, etc. », Paris 1840-42.

(3) Chi desidera prendere particolareggiata conoscenza della struttura di queste parti, consulti i lavori diligentissimi del Lacaze Dethiers "De l'armure génitale des insects ", Ann. des Sc. Nat., 3a serie, 1849-53, vol. 12-1417-18-19. 
l'aiuto di molte figure il passare in rassegna le numerosissime modificazioni delle parti formante l'armatura copulatrice degli insetti. Le principali tuttavia verranno menzionate nella descrizione delle varie famiglie e dei vari generi degli insetti, offrendo esse, nella maggior parte dei casi, importanti caratteri tassonomici.

Nella femmina le parti costituenti l'apparato genitale esterno, che come nei maschi sono modificazioni degli ultimi segmenti addominali, sono destinate principalmente alla deposizione delle uova, e la loro forma varia secondo il modo in cui le uova vengono deposte.

Anche per le femmine ripetiamo ciỏ che si è detto pei maschi, che cioè le principali modificazioni dell'armatura genitale esterna verranno menzionate nella parte descrittiva.

La funzione di riproduzione è esclusiva o quasi degli insetti giunti allo stato perfetto. Ho detto quasi, perchè sono stati menzionati casi di riproduzione avvenuta in insetti allo stato di larva. La cosa venne osservata principalmente dal Wagner in qualche Dittero (1).

La riproduzione ha luogo per lo più poco tempo dopo che l'insetto è uscito dallo stato di ninfa o di crisalide, e s'è trasformato in insetto perfetto.

L'accoppiamento si fa in vari modi e in generale i maschi sono i primi ad essere atti alla riproduzione. Degli organi e delle strutture che favoriscono l'accoppiamento: della scelta sessuale che si esercita o dai maschi o dalle femmine, abbiamo già parlato lungamente nel capitolo $\mathrm{V}$.

L'accoppiamento per lo più segue mentre la femmina è ferma in qualche luogo e in riposo. Qualche volta tuttavia la cosa va altrimenti, e l'accoppiamento ha luogo durante il volo, come è il caso per l'ape, per altri Imenotteri e per qualche Dittero.

Nella maggior parte dei casi il maschio sale sopra la fem-

(1) La specie studiata dal Wagner è la Miastor metraloas, ed appartiene alla famiglia delle Cecidomie. Si consulti a questo proposito il lavoro di Wagner pubblicato negli Ann. d. Sc. Nat. de France, serie 5a, vol. IV, 1865, e gli scritti di Ganine e di BaER pubblicati nelle Memorie dell Accademia delle Scienze di Pietroburgo, 1865. 
mina (nei grilli, come ha osservato il Lespés, avviene spesso l'inverso). In molti Lepidotteri notturni, in molti Rincoti, in vari Ditteri l'accoppiamento ha luogo stando gli insetti sopra uno stesso piano colle estremità dell'addome a contatto.

Si è durante il giorno, o, come nella comune melolonta, in sul far della sera, che ha luogo quasi sempre l'accoppiamento, il quale è di una durata assai varia. Il maschio per lo più muore poco tempo dopo; la femmina vive fin dopo la deposizione delle uova. Questa regola non è tuttavia generale e sono stati osservati casi, soprattutto fra i Coleotteri carabici, in cui i maschi $\dot{e}$ le femmine hanno vissuto per lungo tempo anche dopo avvenuto l'accoppiamento e la deposizione delle uova.

Se l'accoppiamento non ha luogo, molti insetti possono vivere per un tempo relativamente lungo.

Prima di por fine al capitolo intorno alla riproduzione sarà bene accennare ad una maniera di riproduzione importantissima che si osserva non raramente negli insetti, vale a dire la riproduzione partenogenetica.

I comuni gorgoglioni delle rose furono i primi animali in cui venne studiata la partenogenesi. Esaminando in sul finire dell'autunno i rami delle rose carichi di gorgoglioni, si vede che molti individui, i quali sono in parte maschi ed in parte femmine, hanno le ali. Questi individui alati si accoppiano; il maschio muore appena compiuta la fecondazione, la femmina depone le uova sul ramo stesso e poi muore anch'essa. Nella primavera successiva sbucciano dalle uova deposte i giovani gorgoglioni, i quali dopo una diecina di giorni o poco più giungono al loro compiuto sviluppo. Questi individui sbocciati dalle uova deposte in autunno, cosa notevole, sono tutte femmine. Queste femmine partoriscono, senza prima accoppiarsi, non più uova, ma piccoli gorgoglioni già sviluppati, i quali sono pure tutte femmine. Queste si sviluppano e partoriscono pure, senza accoppiarsi, altre femmine le quali alla loro volta giunte al loro compiuto sviluppo partoriscono altre femmine, e cosi di seguito per otto o nove generazioni, le quali durano per tutta la buona stagione. Al sopraggiungere dell'inverno nascono anche i maschi, i quali si accoppiano colle femmine; queste poi fecondate partoriscono non più gorgoglioni, ma uova come abbiamo già detto. 
In qualche altra specie di Rincoti (Phylloxera) le cose si complicano e il ciclo di sviluppo dell'insetto comprende un numero maggiore di forme intermedie fra l'uovo e la forma alata sessuata (1).

Il fenomeno della partenogenesi non è ancora stato spiegato. Ciò che pare certo si è che le riproduzioni vivipare estive si fanno senza che abbia luogo la fecondazione. Si consulti a questo proposito lo scritto di Balbiani, Compt. Rend. de l'Acad. des Scienc., vol. LXII.

Pare anche che la temperatura abbia una grande influenza sulla riproduzione vivipara partenogenetica. Il Kyker (2) infatti potè prolungare di quattro anni il periodo di riproduzione vivipara di femmine tenendo i gorgoglioni al caldo.

(1) Per ciò che è del genere Phylloxera si veda il capitolo dei Rincoti nella parte descrittiva.

(2) «Einige Erfahrungen und Bemerkungen über Blattlaüse Germar Magaz. Entom. », vol. 1, Heft. 2, 1815. 


\section{Bibliografia.}

Gegenbadr, Straus, Leydig, L. Dufour, ecc. Op. citata.

Siebold. - Ueber die Spermatozoen der Crustaceen, Insecten, ecc. Müller's Archiv., 1836.

Stein. - Vergleichende Anatomie und Physiol. der Insekten. Berlino, 1847.

Leuckart. - Micropyle und den feinern Ban der Schalenhaut bei den Insekteneiern. Müller's Archiv., 1855.

Rousset. - Recherches sur les organes génitaux des Insectes Coléopteres de la famille des Scarabéides. Comp. Rend., vol. I, 1860.

Meinert. - Anatomia Forficularum Natur-Tedjzkrift., vol. II, 1865.

Ménznikow. - Embryologische studien an Insecten. Zeitsch. für Wessen. Zool., vol. XVI, 1866.

Claus. - Beobactungen über die Bildung as Insecteneies. Zeit. für wiss. Zool., vol. XIV, 1864.

Ratzeburg. - Die Forst. Insekten.

DoyĖre. - Observ. sur les organes perforants chez les Insectes. Ann. des Sc. Nat., $2^{\text {a }}$ serie, vol. VII, 1837.

J. Perez. - Mémoire sur la parte de l'Abeille reine et la théorie de Dzierzon. Ann. d. Sc. Nat., 6 a serie, vol. VII, 1878.

A. Sanson. - Note sur la Parthénogénèse chez les Abeilles. Ann. d. Sc. Nat., $7^{\text {a }}$ serie, vol. VII, 1878.

Intorno alla struttura dell'apparato copulatore esterno nei vari ordini degli insetti si possono consultare anche tutti gli autori che hanno trattato monograficamente di qualche gruppo d'insetti, ad esempio l'opera del Fischer sugli Ortotteri, quella del Nicolet sulle Pódure, ecc. Si veda la bibliografia che è alla fine di ciascun capitolo della parte descrittiva. 


\section{XIII.}

\section{- SVILUPP0}

L'insetto che sbuccia dall'uovo o che viene espulso dalla madre quando è già pervenuto ad un certo grado di sviluppo subisce, prima di acquistare la forma dei suoi progenitori, vari mutamenti e passa per vari stadi di sviluppo più o meno distinti e più o meno ben caratterizzati fra loro. In questi mutamenti successivi consiste appunto ciò che comunemente si chiama la metamorfosi dell'insetto.

Gli antichi avevano già una qualche cognizione intorno al fenomeno delle metamorfosi, ed Aristotile che, come disse molto bene qualche autore, è un osservatore moderno perduto nell'antichità, ha intorno alla metamorfosi delle farfalle le parole seguenti: - «Le farfalle hanno origine dai bruchi. Si ha da prima un corpicciolo della grossezza di un grano di miglio; un po' più tardi un piccolo verme che a capo di tre giorni diventa un piccolo bruco. Giunti i bruchi al completo loro sviluppo perdono la facoltà di muoversi e cambiano forma. Vengono chiamati allora crisalidi. Queste sono chiuse in un involucro duro e resistente. Quando vengono toccate tuttavia si muovono; inoltre le crisalidi sono chiuse in cavità fatte da una materia analoga ai fili dei ragni. Esse non hanno nè bocca nè altre parti distinte. Poco tempo dopo l'astuccio si rompe e ne esce un animale che vola e che noi chiamiamo farfalla. Nel suo primo stato, quello di larva, egli mangiava ed emetteva escrementi, diventato una crisalide non prende nè rigetta cosa alcuna. E ciò si osserva pure in tutti gli animali che hanno origine dai vermi (l) ».

(1) La parola $\psi u ́ \chi \eta$, significava pei greci farfalla ed anima ad un tempo, e, 
Appena lo spermatozoide passando pel micropilo è giunto nell'interno dell'uovo ed ha dato alla sostanza in questo contenuta quell'impulso speciale nel quale consiste propriamente la fecondazione, comincia nell'interno dell'uovo una serie di mutamenti nei quali consiste il primo periodo di sviluppo dell'insetto.

Lo studio di questi mutamenti sarebbe certamente molto

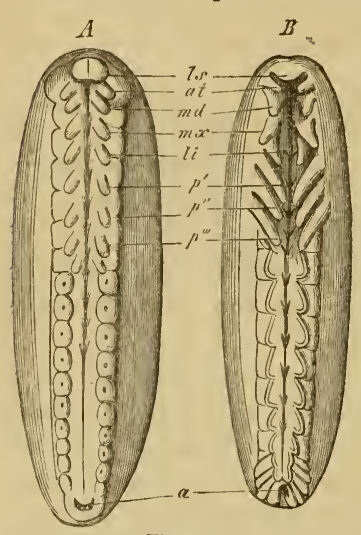

Fig. 42.

Due stadi di sviluppo dell'Hydrophilus piceus. - A stadio di sviluppo non molto inoltrato $-B$ stadio di sviluppo più inoltrato - $l s$ labbro superiore at antenne - $m d$ mandibole $-m x$ mascelle $-l i$ labbro inferiore $-p^{\prime} p^{\prime \prime}$
$p^{\prime \prime \prime}$ zampe.

complesso, noi vediamo che essi possono venire divisi, rispetto alla loro metamorfosi, in tre grandi gruppi che, diciamolo su-

come tutti sanno, poeti e filosofi hanno ripetutamente paragonato lo sviluppo della vita materiale e morale dell'uomo allo sviluppo dell'insetto e principalmente della farfalla. Tutti ricordano i versi di Dante:

Non v'accorgete voi, che noi siam vermi

Nati a formar l'angelica farfalla,

Che vola alla giustizia senza schermi?

Di che l'animo vostro in alto galla?

Voi siete quasi entomata in difetto

Sì come verme, in cui formazion falla.

Purg. X, v. 125. 
bito, non sono tuttavia nettamente separati gli uni dagli altri, ma presentano fra loro, come sempre si osserva nei fatti naturali, una serie più o meno grande di passaggi, di gradazioni, di sfumature.

La prima divisione comprende quegli insetti che si possono considerare come privi di metamorfosi, quegli insetti cioè che nascono già colla forma che debbono tenere per tutta la vita, ed in cui lo stato adulto è contrassegnato soltanto dalla mole e dallo svilupparsi degli organi della generazione. Sono in questo. caso i Tisanuri fra gli Ortotteri.

Non tutti gli Autori tuttavia ammettono questa categoria di insetti senza metamorfosi (immutabilia insecta) : molti li fanno entrare nella categoria seguente.

Il secondo gruppo è quello degli insetti aventi una metamorfosi incompiuta. Gli insetti aventi questo secondo modo di metamorfosi posseggono già nascendo una forma eguale a un dipresso a quella che avranno quando saranno pervenuti allo stato d'insetto perfetto.

Nella mancanza o nello sviluppo più o meno grande delle ali sta la sola differenza fra i vari stadi di sviluppo.

La larva è l'insetto privo di ali.

Nella ninfa, stadio che corrisponde alla crisalide dei Lepidotteri, le ali sono rudimentali.

Nell'insetto perfetto esse sono compiutamente sviluppate. Internamente poi modificazioni importantissime segnano lo stato d'insetto perfetto e queste sono principalmente la modificazione del canal digerente e soprattutto lo sviluppo degli organi della riproduzione, organi che non esistono, meno nei casi che noi abbiamo già visto, nel periodo di larva.

Hanno metamorfosi incompiuta gli Ortotteri ed i Rincoti.

Il terzo gruppo è quello degli insetti che hanno metamorfosi compiute.

Negli insetti appartenenti a questo gruppo i tre stadi di larva, di ninfa e di insetto perfetto, sono sempre molto distinti fra loro e per lo più sono separati da periodi di riposo, periodi che non si osservono negli insetti dalla metamorfosi incompiuta.

Presentano metamorfosi compiuta i Coleotteri, i Ditteri, gli Imenotteri, i Nevrotteri ed i Lepidotteri. 
Esaminiamo, per farci un'idea chiara della metamorfosi, lo sviluppo di un insetto dalla metamorfosi compiuta. Prendiamo una mosca, insetto di cui lo sviluppo può essere studiato facilmente da chiunque.

L'uovo deposto dalla mosca a capo di un giorno o poco più lascia uscire un vermicciattolo il cui corpo è composto di dieci od undici segmenti omonimi, che piglia il nome di larva.

Dopo pochi giorni, se la temperatura è favorevole ed il nutrimento abbondante, la larva ha raggiunto la lunghezza di poco più di un centimetro. In questo stadio mancano le zampe articolate e mancano pure gli occhi, e tuttavia, dirò di passaggio, come risulta da esperimenti fatti dal Pouchet (1), la larva è sensibilissima all'azione della luce. Il canal digerente è relativamente molto sviluppato, e gli organi della riproduzione mancano al tutto.

Giunto l'animale alla lunghezza di un centimetro e mezzo circa, cessa dal mangiare; il tessuto adiposo si sviluppa in grande quantitá e riempie a poco a poco quasi tutte le cavità del corpo.

La larva in questo periodo di sviluppo se è sulla terra molle vi si affonda e rimane immobile per un certo tratto di tempo. I segmenti allora si avvicinano, si saldano fra loro in modo che la larva viene ad avere l'aspetto di un uovo allungato di color bruniccio, dovuto al colorarsi del tessuto chitinoso che dapprima formava l'integumento della larva.

Se noi apriamo l'involucro esterno, paragonabile in certo modo al bozzolo dei Lepidotteri, noi non troviamo più nulla della struttura della larva, non più canal digerente, non più nervi, non più trachee, ecc., ma troviamo semplicemente una poltiglia giallognola alquanto densa separata da alcuni tramezzi membranosi.

Aprendo invece il bozzolo verso il quarto o il quinto giorno troviamo già abbozzati, per cosi dire, gli organi dell'insetto perfetto; aprendolo invece verso il settimo o l'ottavo troviamo addirittura una mosca quasi al tutto sviluppata.

(1) Mém. de 7'Acad. des Scienc., 1870. Guerin Menevilue, Revue de Zool., 1871-72, vol. XXIII. 
L'insetto perfetto esce dal bozzolo sollevando, nella maggior parte dei casi, a mo' di cappuccio la porzione anteriore del bozzolo stesso.

Nel periodo di ninfa, secondo gli studi più recenti, vi sarebbero un periodo di disorganizzazione ed un periodo di riorganizzazione in guisa che la ninfa potrebbe essere considerata come un secondo uovo.

Ciò che io ho detto per lo sviluppo della mosca lo si puó ripetere anche in generale per gli altri insetti: variano i particolari, ma il piano dello sviluppo rimane lo stesso.

In molti casi, soprattutto fra i Lepidotteri e gli Imenotteri, il periodo di ninfa o di crisalide è distinto per la costruzione di un bozzolo speciale.

Si veda per maggiori particolari intorno alla metamorfosi dei singoli ordini degli insetti la parte descrittiva.

Rispetto al tempo che l'insetto impiega a percorrere i vari stadi di sviluppo non si può dire nulla di generale.

In qualche specie il periodo di sviluppo dura pochi giorni, in altri invece dura parecchi anni. In generale tuttavia esso si compie nello spazio di un anno. Le condizioni di temperatura, di umidità, ecc., hanno molta influenza sulla durata dello sviluppo (1).

Riassumendo, noi possiamo dire che la vita degli insetti consta in generale di due grandi periodi, vale a dire:

$1^{\circ}$ Di un periodo di nutrizione in cui è principalmente sviluppato il canal digerente, in cui mancano gli organi della riproduzione e che comprende gli stadi di larva e di ninfa.

$2^{\circ} \mathrm{Di}$ un periodo di riproduzione in cui il canal digerente è relativamente poco sviluppato (qualche specie anzi non si nutre affatto), e sono invece sviluppati gli organi della riproduzione e che comprende l'insetto perfetto.

Ci rimane da dire qualche cosa ancora intorno ad una maniera speciale e molto complessa di sviluppo non ben conosciuta infino ad oggi, che si osserva in varie specie di Coleotteri appartenenti alla famiglia dei vescicantidi.

(1) Si consulti a questo proposito GHulian1: «Influenza del calore asciutto sullo sviluppo di alcune crisalidi », Bull. Soc. Ent. Ital., anno V, 1873. 
Vediamo (colla scorta del Fabre che per primo studiò questa maniera di sviluppo), come vanno le cose nella Sitaris humeralis.

Dopo la fecondazione la femmina della specie citata va a portare le uova nei condotti scavati nella terra da varie specie di Imenotteri solitari. Da queste uova sbucciano delle piccole larve lunghe poco più di un millimetro, agilissime, aventi quattro occhi, mandibole robuste e lunghe zampe.

Queste larve passano l'inverno presso l'imboccatura dei canali citati. Al sopraggiungere della primavera gli Imenotteri maschi, che sono i primi, secondo il solito, a svilupparsi, escono dal nido, e nel loro passaggio raccolgono un numero vario di larve di sitaris, le quali si aggrappano ai loro folti peli. I maschi sopradetti andando qua e là pei fiori lasciano qua e là le larve che essi portano. Gli Imenotteri femmine trasportano nello stesso modo inconsciamente, pigliandole dai fiori dai quali vanno a suggere il nettare, le larve della sitaris nei loro nidi.

La femmina deponendo in ciascuna cella del nido un uovo vi lascia cadere inavvertentemente anche qualcheduna delle larve che ha portate con sè. La piccola larva della sitaris fora l'uovo, ne mangia il contenuto e nell'interno del guscio subisce una muta della pelle. Ne esce allora una seconda larva che differisce dalla prima per avere le zampe e le mandibole appena sviluppate e per mancare di occhi. Questa larva si nutre del miele che tappezza la cella. Dopo qualche tempo la pelle si dissecca e l'insetto piglia la forma di una ninfa. L'animale entra cosi nel terzo stadio di sviluppo, stadio che viene distinto col nome di pseudoninfa. Passa in questo stadio senza nutrirsi e senza muoversi l'inverno. Nella primavera successiva sbuccia una nuova larva simile alla seconda, la quale dà origine ad una ninfa propriamente detta, in cui si trovano già accennate, secondo il solito, le forme dell'insetto adulto, dalla quale finalmente si sviluppa l'insetto perfetto.

Le larve delle sitaris sono quindi nel primo periodo del loro sviluppo carnivore, nel secondo invece mellivore.

Un ciclo analogo di sviluppo si osserva pure nelle meloe e nei Mylabris. 


\section{Bibliografia.}

Réaumur, Roesel, Swammerdam, De Geer, Welsmanx, ecc. Op. citate.

N. Bobretzky. - Ueber die Bildung des Blastoderms und der Keimblätter bei den Insecten. Zeit. für wiss. Zool., 1878.

Gaxis. - Ueber die Embryonalhülle der Hymenopteren und Lepidopterenembryonen. Mém. de l'Acad. de St-Petersbourg, $7^{\text {a }}$ serie, vol. XIV.

A. KoELliker. - Observationes de prima Insectorum genesi, ecc. Dissert. Inaug. Turici, 1842.

A. Weismanx. - Ueber die Enstehung des vollendeten Insekts Larve und Puppe, ecc. Frankfurt, 1863.

- Die Entwickelung der Dipteren im Ei, ecc. Zeit. für wiss. Zool., vol. XIII.

- Die nachembryonale Entwickelung der Musciden, ecc. Zeit. für wiss. Zool., vol. XIV.

G. Blanchard. - Métamorphoses des Insectes. Paris, 1868.

M. Girard. - Les métamorfoses des Insectes. Paris, 1867.

Favre. - Mémoire sur l'hypermétamorphoses et les mœurs des Méloides. Ann. des Sc. Nat., 4a serie, vol. VII, 1857, e vol. IX, 1859.

- Notes sur quelques points de l'histoire des Cerceris, des Bembex, des Sitaris, ecc. Ann. des Sc. Nat., serie 4a, vol. VI, 1856.

- Observations sur les mœurs des Cerceris, ecc. Ann. des Sc. Nat., 4 a serie, vol. IV, 1855. 


\section{XIV.}

\section{CLASSIFICAZIONE}

Una cinquantina d'anni fa si aveva, o almeno si credeva di avere, un concetto giusto e chiaro della specie. Vi sono tante specie, si diceva, quante sono state create e queste sono fisse ed immutabili. Questo concetto della specie era comodo al naturalista classificatore, inquantochè gli concedeva di classificare gli animali tenendo conto del puro e semplice esame morfologico delle parti esterne senza curarsi nè dell'interna struttura nè dello sviluppo degli animali stessi.

Al giorno d'oggi le teorie Darwiniane nello stesso tempo che portarono luce vivissima intorno ad un grande numero di fatti relativi agli esseri viventi, cambiarono anche al tutto il concetto della specie. Le ricerche poi del Müller, del Gegenbaur, del Wallace, dell'Haekel, ecc., intorno alle metamorfosi, alle generazioni alternanti, alla metagenesi, al polimorfismo, ecc., hanno pure contribuito moltissimo a dimostrare erroneo l'antico concetto della fissità della specie (1).

Al giorno d'oggi una soddisfacente definizione della specie non è possibile. Quello che è certo oramai si è che la specie non ha limiti precisi, che la specie è variabile. Le varietà quindi non sono altro che specie in via di formazione, e le specie non sono altro che varietà, le quali, come si dice ordinariamente, si sono fissate, vale a dire che sono riuscite vincitrici nella lotta per la vita, mentre tutte le altre varietà, che le - collegano colla specie madre (2), sono perite.

(1) Si consulti a questo riguardo G. CANestrins, La teoria dell'evoluzione. Torino, 1877.

(2) Quantunque la teoria della variabilità della specie sia oramai stata accet- 
Io non intendo di fare qui la storia delle molte classificazioni degli insetti che la scienza possiede da Aristotile ai nostri giorni, imperocchè questo studio, sebbene interessantissimo, ci porterebbe troppo fuori dal nostro campo. Io mi limito a riunire sotto forma di quadro le classificazioni più spiccate e che meglio caratterizzano il cammino della scienza, avvertendo che le classificazioni moderne (quella del Claus in special modo) differiscono dalle antiche in ciò che esse procedono dal semplice al complesso, e raggruppano le forme animali, tenendo conto soprattutto della loro ontogenia e del loro sviluppo, elementi che erano, or non è gran tempo ancora, come ho detto, al tutto trascurati.

tata dai più insigni naturalisti, alcuni tuttavia non vogliono ammetterla. Fra questi è il signor Maurizio Girard; ecco ciò che si legge a pagina 215-216 del primo volume del suo Traité élémentaire d'Entomologie (libro pregievolissimo sotto altri rispetti) (Parigi, 1873): - «Quelques difficulté que l'esprit humain éprouve à comprendre des espèces créés de toutes pièces, et une fois pour toutes, c'est ancore la seule hypothèse qui conduise à des conséquences extrêmes raisonnables et justifiées par les faits. Il faut supposer seulement que l'espèce est susceptible de variations limitées, assez étendues même dans certains cas, conservant toutefois le plan fondamental du Créateur. Si l'on ne restreint pas les idées de modification e de passage à ces variations d'une seule espèce, on peut, de proche en proche, arriver aux conclusions les plus absurdes, et, avec un peu de bonne volonté, faire descendre l'homme non seulement du Singe mais du Trilobite ou de la Monade». 


\begin{tabular}{|c|c|}
\hline 憘 & 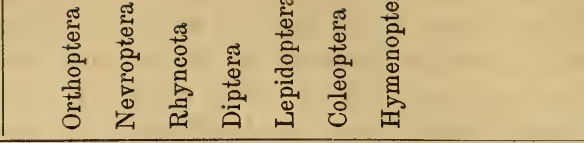 \\
\hline$g$ & 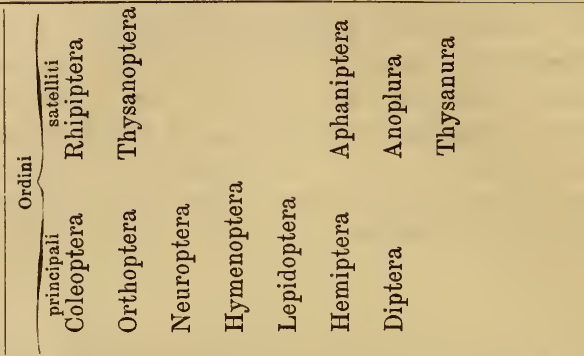 \\
\hline 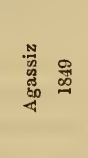 & 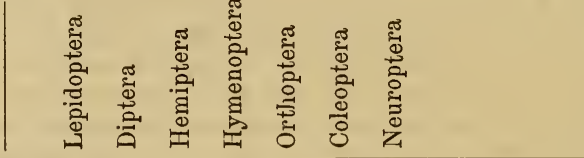 \\
\hline 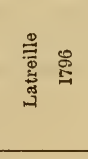 & 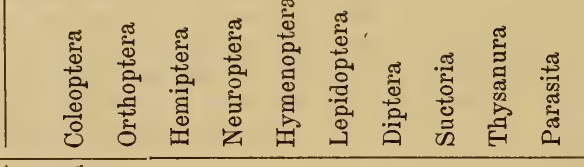 \\
\hline 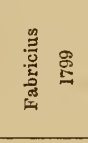 & 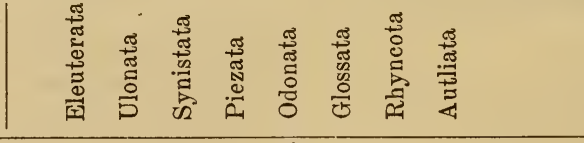 \\
\hline 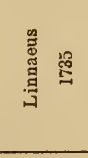 & 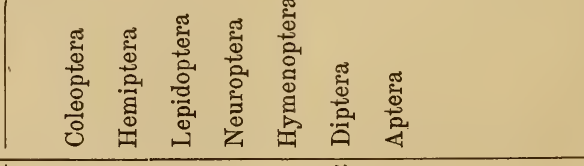 \\
\hline 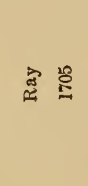 & 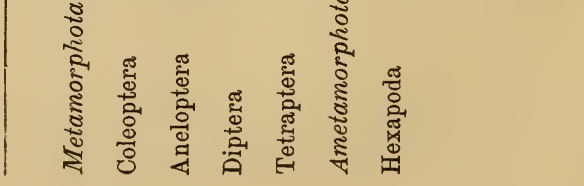 \\
\hline
\end{tabular}


La classificazione che noi seguiamo in questo lavoro è quella del Claus. Io credo che sia utile dal punto di vista pratico per maggior chiarezza di fare precedere agli ordini una tavola sinottica degli ordini stessi, ed a ciascun ordine una tavola dicotomica delle famiglie più importanti.

\section{Tavola sinottica degli ordini.}

I. Ali in numero di due. Il secondo paio è rappresentato dai due bilancieri

II. Ali in numero di quattro.

A. Il paio anteriore di consistenza cornea, trasformato in due elitre

$A^{\prime}$. Tutte quattro le ali membranose.

$B$. Apparato boccale foggiato per masticare o per lambire.

a. Protorace non saldato ai segmenti toracici seguenti:

b. Metamorfosi incompiute: le due ali hanno in generale nervature dissimili . . . Ortotteri.

$b^{\prime}$. Metamorfosi compiute: le nervature delle ali formano una fitta rete. . . . Nevrotteri.

$a^{\prime}$. Protorace saldato ai segmenti toracici seguenti : apparato boccale foggiato per lo più a lambire. Imenotteri.

$B^{\prime}$. Apparato boccale non foggiato per masticare nè per lambire.

a. Parti della bocca trasformate in una tromba succiante: le ali sono ricoperte da un grande numero di variopinte squamette: protorace saldato ai segmenti toracici seguenti: metamorfosi compiute . . . . Lepidotteri. $a^{\prime}$. Parti della bocca foggiate per pungere e per succiare. Protorace non saldato ai segmenti toracici seguenti: metamorfosi incompiute . . . Rineoti. 


\begin{tabular}{|c|c|c|c|c|c|}
\hline Ordini & Metamorfosi & ব & $\underset{\text { Struttura }}{\text { Ali }}$ & $\begin{array}{c}\text { Apparato } \\
\text { boccale }\end{array}$ & $\begin{array}{c}\text { Proto- } \\
\text { race }\end{array}$ \\
\hline Ortotteri & incompiute & 4 & $\begin{array}{l}\text { Quasi sempre tutte } \\
\text { quattro membra- } \\
\text { nose }\end{array}$ & $\begin{array}{l}\text { atto a ma- } \\
\text { sticare }\end{array}$ & libero \\
\hline Nevrotteri & compiute & 4 & $\begin{array}{l}\text { Membranose e con } \\
\text { numerose e fitta- } \\
\text { mente intrecciate } \\
\text { nervature }\end{array}$ & $\begin{array}{l}\text { atto a ma- } \\
\text { sticare }\end{array}$ & libero \\
\hline Rincoti & incompiute & 4 & $\begin{array}{l}\text { Quasi sempre intie- } \\
\text { ramente membra- } \\
\text { nose }\end{array}$ & $\begin{array}{l}\text { atto a pun- } \\
\text { gere e suc- } \\
\text { ciare }\end{array}$ & libero \\
\hline Ditteri & compiute & 2 & Membranose & $\begin{array}{l}\text { atto a pun- } \\
\text { gere ed a } \\
\text { succiare }\end{array}$ & saldato \\
\hline Lepidotteri & compiute & 4 & $\begin{array}{l}\text { Ricoperte da minu- } \\
\text { te squame vario- } \\
\text { pinte }\end{array}$ & $\begin{array}{l}\text { atto a suc- } \\
\text { ciare }\end{array}$ & saldato \\
\hline Coleotteri & compiute & 4 & $\begin{array}{l}\text { Le due anteriori du- } \\
\text { re e resistenti (e- } \\
\text { litre) }\end{array}$ & $\begin{array}{l}\text { atto a ma- } \\
\text { sticare }\end{array}$ & libero \\
\hline Imenotteri & compiute & 4 & $\begin{array}{l}\text { Membranose nerva- } \\
\text { ture poco nume- } \\
\text { rose }\end{array}$ & $\begin{array}{l}\text { atto a lam- } \\
\text { bire ed a } \\
\text { masticare }\end{array}$ & saldato \\
\hline
\end{tabular}




\section{Bibliografia.}

Oltre alle opere ripetutamente citate di Réaumur, di Roesel, di De Geer, di Linneo, di Fabricio, ecc., si consultino pure le seguenti:

Latreille. - Précis des caractères génériques des Insectes, 1796.

- Familles naturelles du Règne animal, 1825.

- Cours d'Entomologie, 1831.

BAER. - Beitrage zur Kentniss der niederen Thiere. Nova Acta Acad. Leopold. Carolin., XIII, 1827.

Kirby e Spexce. - An Introduction to Entomology. London, 1828. Varie edizioni.

- Handbuch der Entomologie. Berlino, 1832-55.

Cuvier. - Le Règne animal distribué d'après son Organisation, etc. Paris 1849. Deméril A. M. C. - Considérations générales sur la classe des Insectes, où l'on traite du rang que les Insectes paraissent avoir occupé dans l'échelle des êtres; de leur classification, de leur distribution en genres. Strasbourg et Paris, 1823.

- Entomologie analytique. Histoire générale. Classification naturelle et méthodique des Insectes à l'aide de tableaux synoptiques. Paris, 1860.

WeSTWOoD J. 0. - The entomologist's text-book: an introduction to natural history, structure, physiology and classification of Insects. London, 1838.

- An Introduction to the modern classification of Insects founded on the natural habits and correspondig organisation of the different families. London 1839-1840.

V. Carus e C. Gestaecker. - Handbuch der Zoologie. Leipzig, 1868-1875.

C. Clauss. - Grundzüge der Zoologie. Marburg und Leipzig, 1876.

M. Grirard. - Traité elémentaire d'Entomologie. Paris, 1873-1879 (in corso di pubblicazione). 


\section{XV. \\ ORTOTTERI}

Insetti con apparato boccale foggiato per masticare. Metamorfosi incompinte.

Linneo collocava gli Ortotteri (meno le Forficole che egli metteva insieme ai Coleotteri) coi Rincoti. Gli Ortotteri costituivano per lui la divisione dei Rincoti masticatori. Il De Geer pel primo cominciò a formare degli Ortotteri una divisione speciale col nome di Dermatteri. Fabricio poi caratterizzandoli meglio e più esattamente li distinse col nome di Ulonata. L'Olivier finalmente cambio il nome di Dermatteri e di Ulonata in quello di Ortotteri (ali diritte) nome che è in uso anche al giorno d'oggi. L'ordine degli Ortotteri è il meno naturale di tutti gli ordini degli insetti e quindi (per le svariate forme che esso racchiude) riesce cosa difficilissima il darne un'idea complessiva, chiara e precisa.

Il capo negli Ortotteri è sempre relativamente molto sviluppato, ora convesso e poco distinto dal corpo come nei grillidi, ora molto allungato ed appuntito anteriormente come nelle Truxalis, ora è appiattito, allargato trasversalmente e ben distinto dal corpo come nelle Mantis.

Le antenne non mancano mai e ora sono filiformi e constano di pochi articoli come nei Tisanuri, ora invece ne hanno un numero grandissimo e misurano in larghezza anche il doppio della lunghezza del corpo come si osserva in molti Locustini, ora sono appiattite come nelle Truxalis.

Gli occhi sono quasi sempre ben sviluppati, qualche volta tuttavia mancano, talora invece sono piccolissimi e ridotti, come nei Tisanuri, a semplici ocelli. Sono invece molto svilup- 
pati in quasi tutti i Pseudonevrotteri, ed in molti Ortotteri genuini, ad esempio nelle Mantis.

L'apparato boccale è foggiato per masticare e per mordere, consta quindi di tutte quelle parti che noi abbiamo detto essere solite trovarsi in questo caso (Capit. XI). Noteró tuttavia che è caratteristica dell'apparato masticatore degli Ortotteri la galea (mascella esterna secondo Erichson, palpo mascellare interno secondo Burmeister), che può essere considerata come un lobo esterno od anche come una gengiva esterna (Fabricio) della mascella.

Il grado di sviluppo dell'apparato masticatore varia nei diversi sottordini. Questo è poco sviluppato ed in qualche caso anzi al tutto rudimentale nei Tisanuri: molto sviluppato invece e provviste di robuste dentellature (1), negli Ortotteri genuini e nella massima parte dei Pseudonevrotteri. Raramente, si noti, negli Ortotteri le mandibole, soprattutto dei maschi, ci presentano, come arviene frequentemente nei Coleotteri, uno sviluppo anormale. Presenta mandibole sviluppatissime fra gli Ortotteri il genere Anostostoma della Nuova Olanda.

Il torace ora è appiattito, circolare, clipeiforme come nelle blatte; ora è carenato, allungato, con lobi e dilatazioni laterali più o meno sviluppate, come nelle Mantidi; ora è corto, quadrato od ovale, come nei grili, ecc. Il protorace è sempre libero e mobile.

Le zampe sono negli Ortotteri, nella massima parte dei casi, ben sviluppate, e sono per lo più lunghe e sottili; i tarsi possono constare di due, tre (caso più frequente) di quattro ed anche di cinque articoli. Le anteriori possono essere foggiate, come nella Grillotalpa, per scavare, ovvero come organi di presa, come nelle Mantidi. Le posteriori sono quasi sempre più lunghe e più robuste delle altre e servono al salto.

Le ali mancano interamente nei Tisanuri ed in varie specie di Locustini. Negli altri Ortotteri possono essere di varie guise.

(1) Marcel de Serres paragona queste dentellature ai denti dei mammiferi e trova che si possono dividere in incisivi, canini e molari. Questa comparazione è forzata, i denti delle mandibole degli Ortotteri non avendo anatomicamente nulla che fare con quelli dei vertebrati. 
Nelle forficule, ad esempio, il primo paio è trasformato in elitre ed è quindi molto diverso dal secondo che è membranoso.

Negli Ortotteri propriamente detti le due paia di ali differiscono fra loro nella disposizione delle nervature. Nei Pseudonevrotteri invece le quattro ali sono, perció che è delle nervature, generalmente eguali fra loro.

Molto variabile eziandio è la forma dell'addome, il quale conta dieci o al più undici segmenti. Ora è corto e grosso come nei grilli, ora allungatissimo e sottile come nei Bacillus e nei Phasma, ecc. Gli ultimi segmenti addominali portano per lo più nella femmina un ovopositore, e nel maschio delle appendici articolate che gli servono per tener ferma la femmina durante l'accoppiamento. L'addome dei Japyx, come io ho gia fatto notare, presenta delle zampe articolate rudimentali.

Molto lungo, in generale, è negli Ortotteri il canal digerente, anche ben sviluppate sono per lo più le ghiandole salivari. Molto numerosi sono i canali Malpighiani. Non è relativamente molto grande la centralizzazione del sistema nervoso; i gangli addominali variano da cinque a sette. I sessi si distinguono fra loro per la presenza di caratteri sessuali secondari per lo più ben spiccati.

Le metamorfosi degli Ortotteri sono incompiute, e il loro sviluppo si compie ora sulla terra, e in questo caso mutano, in generale, quattro o cinque volte la pelle (1) come nei grilli, nelle Mantis, negli Ortotteri genuini, ecc.; ora invece nell'acqua come in quasi tutti i Pseudonevrotteri. La madre, meno nelle Forficole ed in poche altre specie, non si dà alcun pensiero delle uova e della prole. Gli Ortotteri si nutrono quasi esclusivamente di sostanze vegetali; presentano il fenomeno delle migrazioni, e mostrano in molti casi un molto spiccato mimetismo. Gli Ortotteri comprendono i giganti degli insetti (Phasma, lung. da 0,30 a 0,40 ), e sono sparsi per tutta la terra, abbondando naturalmente di più nelle regioni equatoriali. Non salgono molto alto sui monti. Gli Ortotteri fanno

(1) Nei nostri paesi molti Ortotteri compiono la loro ultima metamorfosi e ci appaiono allo stato perfetto sul finir dell'estate o sul principio dell'autunno. 
vita terraiola meno tuttavia qualche genere esotico della categoria dei Tettix, ed il genere Prisopus fra i Fasmidi che vivono nell'acqua. Quantunque gli Ortotteri, relativamente ai Coleotteri ed ai Lepidotteri, siano ancora poco studiati, possiamo dire tuttavia che essi sono molto meno numerosi in ispecie dei due ordini ora menzionati.

\section{Tavola di classificazione degli Ortotteri.}

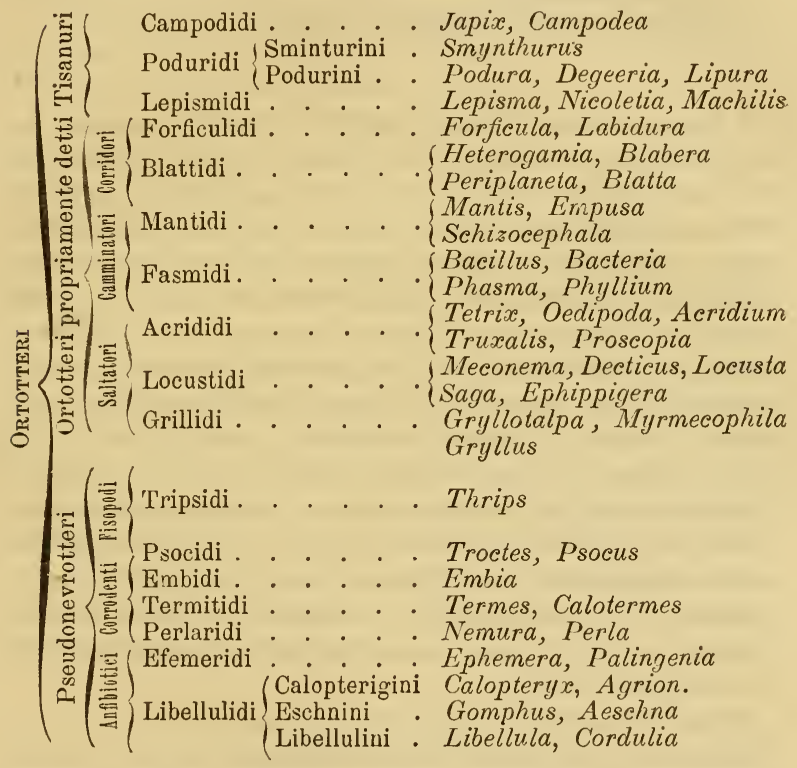

A. Corpo peloso o ricoperto di scaglie. Senza ali. Tisanuri. $A^{\prime}$. Corpo senza peli. Quattro ali.

a. Ali anteriori strette e dure, talvolta trasformate in elitre: ali posteriori membranose: le nervature variano nelle due paia di ali . . . . . Ortotteri genuini. $a^{\prime}$. Ali tutte quattro membranose: le nervature non variano gran fatto nelle due paia di ali . Pseudoneorotteri. 


\section{Tisanuri.}

A. I segmenti addominali presentano rudimenti di zampe. Addome di 10 segmenti. Forma generale del corpo allungata e che ricorda quella dei Iulus . . . . . Campodidi.

$A^{\prime}$. I segmenti addominali non hanno rudimenti di zampe.

a. Mascelle senza palpi : mandibole nascoste. Poduridi. b. Corpo quasi sferico . . . . Sminturini. $b^{\prime}$. corpo allungato . . . . . Podurini. $a^{\prime}$ Palpi mascellari composti di cinque a nove articoli.

Lepismidi.

I Tisanuri hanno il corpo ricoperto di peli o di squamette. Ora hanno forma allungata, ora invece globosa. Mancano le ali. L'addome, che conta al più cinque segmenti, presenta posteriormente un'appendice ora forcuta alla sua estremità inferiore, ora semplice, che allo stato di riposo è ripiegata sotto l'addome. L'animale salta facendo scattare ad un tratto questa appendice, la quale come una molla spinge in alto l'insetto stesso; in qualche caso $(J a p y x)$, come ripetutamente ho avuto occasione di far osservare, vi hanno inferiormente dei rudimenti di zampe. L'apparato boccale è poco sviluppato. Per lo più si trovano solamente ocelli. Non hanno metamorfosi. I Tisanuri sono di mole molto piccola e vivono quasi esclusivamente nei luoghi umidi, sotto alle pietre, nei prati, appiè dei muri o degli alberi, sotto il legno marcio, nel letame, ed in generale nelle materie animali e vegetali in decomposizione. Resistono molto al freddo e qualche specie si spinge sui monti fin sugli alti nevai.

Spesso alcune specie soprattutto dei generi Achorutes Templ., Podura L. e Isotoma Baurl., sviluppatesi in gran numero nelle foreste che coprono i primi contraforti Alpini, vengono da un colpo di vento trasportate al piano ricoprendo vaste estensioni di terreno di una polvere nerognola saltellante e che incute terrore alle superstiziose popolazioni. Un fatto simile avvenne qualche anno fa nelle Langhe in Piemonte.

Fino ad ora non vennero studiate dei Tisanuri che le specie Europee e qualche specie americana.

I Tisanuri segnano il passaggio fra gli insetti ed i miriapodi 
e potrebbero per molti caratteri (come vogliono alcuni autori) formare un ordine distinto.

Ricordiamo fra gli altri i generi seguenti:

Iapyx Hal. privo di occhi, Campodea Westw.

Smynthurus Latr. corpo corto e quasi sferico dai segmenti, salvo quelli del torace, saldati insieme; articoli terminali delle antenne piccolissimi.

Podura L. Corpo allungato, segmenti non saldati e antenne corte.

Degeeria Nic. Antenne con quattro articoli, corpo ricoperto di peli claviformi. La $D$. nivalis L. è quella che più frequentemente incontrasi sugli alti nevai alpini.

Lepisma L. Addome terminato da tre filamenti: palpi con cinque articoli, corpo ricoperto da squamette elegantemente colorite.

La specie più comune e la $L$. saccarina $L$. che vive nelle nostre case, fra i fogli dei libri vecchi e poco usati, e fra le fessure del legno dei telai delle finestre, e che venne, pel suo corpicciolo argenteo e per la rapidità dei suoi movimenti, chiamata volgarmente pesciolino d'argento. Secondo il Geoffroy questa specie si nutrirebbe degli Psocus e di altri piccoli insettucci; altri invece vogliono si nutra semplicemente del legno tarlato o di zucchero e di altre sostanze analoghe. Secondo il Latreille la L. saccarina sarebbe originaria dell'America.

Nel genere Nicoletia Gerv. mancano al tutto gli occhi.

Il genere Machilis Latr. va distinto per avere gli occhi composti e grandi. Le specie di questo genere abitano i luoghi umidi e non sono rare fra noi.

\section{Ortotteri genuini.}

A. Zampe atte al correre, tutte eguali fra loro e non molto sviluppate.

a. Corpo allungato: le due ali anteriori corte e foggiate a mo' delle elitre dei Brachelitri fra i Coleotteri. Tarsi con tre articoli

Forficulidi.

$a^{\prime}$. Corpo appiattito: le due ali anteriori grandi un po' incrociate fra di loro e analoghe per la loro consistenza alle elitre. Tarsi con cinque articoli . . . . Blattidi. 
$A^{\prime}$. Zampe molto sviluppate in lunghezza o tutte eguali fra loro

o le anteriori foggiate ad organi di presa.

a. Le zampe anteriori molto più sviluppate che non le mediane e le posteriori e foggiate ad organi di presa: corpo allungato. . . . . . . Mantidi.

$a^{\prime}$. Le zampe anteriori non sono foggiate ad organi di presa: corpo allungato, filiforme, ali rudimentali per lo più, o mancanti . . . . Fasmidi.

$A^{\prime \prime}$. Zampe posteriori più ingrossate delle mediane e delle anteriori

e foggiate pel salto (1).

a. Corpo allungato e compresso lateralmente.

$b$. Tarsi con tre articoli: ali anteriori dure e di poco più larghe delle posteriori, le quali sono nello stato di riposo ricoperte dalle prime . . . . Acrididi.

$b^{\prime}$. Tarsi con quattro articoli: protorace selliforme, ovopositore della femmina molto sviluppato ed allungato a mo' di sciabola . . . . . . Locustidi.

$a^{\prime}$. Corpo tozzo cilindrico: tarso con tre articoli: elitre corte e che non ricoprono intieramente le ali posteriori. Grillidi.

Forficulidi.

Gli Ortotteri di questa famiglia ricordano per la forma del loro corpo i Brachelitri fra i Coleotteri., Il corpo è allungato: le ali, in numero di quattro, sono le anteriori dure e foggiate a mo' di due elitre, sempre più corte dell'addome, il quale resta cosi quasi totalmente scoperto, e le posteriori membranose e ripiegate in un modo speciale al disotto delle prime; in qualche caso le ali o sono rudimentali, o mancano al tutto, come si osserva in varie forme del gruppo delle Chelidure: le zampe sono corte e tutte eguali fra loro. L'addome è allungato ed appiattito, meno che nel genere Cylindrogaster, in cui è cilindrico: ora ha dimensioni eguali alla base ed all'apice, ora invece ingrossa (Chelidura) posteriormente. Il numero dei segmenti è per lo più di nove. L'ultimo segmento addominale va distinto per la presenza di un organo speciale, detto pinza dalla sua forma. Le due aste simmetriche (branche), che la costi-

(1) Fa eccezione il genere Pneumora Thnbg. (P. ocellata) dell'Africa meridionale appartenente agli Acrididi per molti caratteri, il quale non ha le zampe posteriori ingrossate ed atte al salto. 
tuiscono, variano moltissimo nella forma. In generale la pinza è molto più sviluppata nei maschi che non nelle femmine. Il Burmeister crede che la pinza corrisponda ai cercini anali degli altri Ortotteri.

Il capo è mediocremente sviluppato; le antenne sono filiformi, ed hanno un numero di articoli che varia da dodici a quaranta. Il numero degli articoli delle antenne ha molta importanza nella classificazione delle Forficule. Il capo non porta ocelli.

Il canal digerente non è molto lungo ed in qualche caso (Labidura, ecc.) è accompagnato da ghiandole salivari più o meno sviluppate.

I Forficulidi, per quanto se ne sa oggi, mutano quattro volte la pelle prima di giungere allo stato d'insetto perfetto. Le larve e le ninfe si distinguono dall'insetto perfetto principalmente per non avere le elitre e le ali.

Non molto spiccati sono i caratteri sessuali secondari.

. I colori dei Forficulidi sono poco vivaci e variano dal bruno rossiccio.intenso al bruno chiaro, al giallognolo, più o meno intenso: raramente presentano delle macchiettature. I Forficulidi vivono spesso riuniti in gran numero assieme, nascosti sotto le scorze degli alberi, o nel terreno, o sotto alle foglie cadute fuori del contatto della luce (1). Sono, come in generale tutti gli Ortotteri, molto voraci, e si nutrono per lo più di sostanze vegetali (2). Sono ghiotti in special modo della polpa dei frutti ( $F$. auricularia) e del nettare dei fiori. Quando si sviluppano in troppo grande numero, possono tornare dannosissimi alle frutta (3).

(1) Erroneamente il volgo crede che la Forbicina (Forficula auricularia) penetri nell'orechio dell'uomo e possa produrre danno colle mandibole all'orecchio stesso. Può accadere che dormendo, ad esempio, o sull'erba di un prato, o in un giardino sotto ad un albero da frutta, una qualche forbicina, per ripararsi dalla luce, penetri nell'orecchio; ma è una cosa puramente accidentale e in ogni caso è fatto di veruna conseguenza.

(2) Non sdegnano tuttavia le sostanze animali, come già osservarono il Latreille ed il Dufour, e si nutrono, come ho avuto agio di osservare ripetutamente io stesso nella $F$. auricularia o nella riparia, molto volentieri dei cadaveri di altri insetti ed anche di individui della loro stessa specie.

(3) Il Kirby avendo trovato delle forficule nelle spiche di grano, crede che di- 
I Forficulidi non giungono a grande mole e sono sparsi per tutta la terra: abbondano naturalmente nelle regioni calde. Salgono sui monti ai duemila metri e più sul livello del mare.

Sono degni di nota i generi seguenti:

Apachya Aud-Serv. Questo genere, che comprende specie delle Indie orientali, va distinto pel grande appiattimento del corpo. Le specie di questo genere (A. depressa, A. chartacea, ecc.) vivono sotto la scorza degli alberi.

Il genere Cylindrogaster è notevole pel suo addome cilindrico; è proprio del Brasile.

Proprio delle regioni calde dell'Asia orientale e dell'Africa è il genere Echinosoma dal corpo spinoso. E. afrum, E. horridum).

Labidura. Questo genere, che è cosmopolito, ha l'aspetto della forficola comune; il numero degli articoli delle antenne

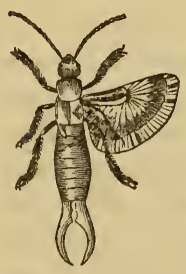

Fig. 43. è variabile da venti a trenta: in complesso la mole delle specie di questo genere supera quella delle forficole propriamente dette. La specie più conosciuta è la L. riparia Pallas o L.gigantea Fabr. Non è rara in Italia.

Brachylabis Dohrn. Le elitre sono poco sviluppate $o$ al tutto rudiméntali. Varie specie di questo genere abitano le coste del Mediterraneo. Forficula auricularia. non sono rare sulle coste italiane.

Il genere Labia Leach comprende le forme più piccole di Forficulidi. È specie comune la L. minor Linn.

Forficula Linn. Le antenne di questo genere molto ricco di specie contano dieci o al più quindici articoli : le elitre sono in generale ben sviluppate. La $F$. auricularia è la specie che più comunemente si trova in Europa. Oggidi non è rara anche nell'Africa settentrionale, nell'isola di Madera ed in qualche regione delle Indie Orientali: probabilmente la diffusione di questa specie è dovuta all'azione dell'uomo.

vorino i Thrips. Io ho osservato ripetutamente nel contorno di Torino, che le spiche di grano in certi campi sono, soprattutto verso sera, in sul principio di giugno cariche di Forficula auricularia; ma non ho mai potuto determinare con certezza la ragione di questo fatto. La cosa merita di essere studiata ancora. 
Il genere Chelidura Latr. è caratterizzato principalmente dal suo addome dilatato posteriormente. Le specie di questo genere fino ad ora state descritte sono tutte europee ed abitano le regioni montagnose. È un genere del resto fino ad oggi poco studiato.

Blattidi.

Si distinguono i Blattidi dagli altri Ortotteri principalmente pel loro corpo appiattito ed ovale. Il capo è mediocremente sviluppato e nascosto in buona parte dal pronotum. Le antenne sono filiformi e di lunghezza variabile nelle diverse specie ed anche spesso nei sessi della stessa specie. Raramente vi hanno ocelli sul capo (maschi del genere Heterogamia). Le mandibole sono molto robuste e sviluppate. Il torace è appiattito e per lo più molto sviluppato. L'addome è quasi sempre di forma più ovale nelle femmine che non nei maschi. In questi ultimi si contano sette segmenti compiuti, un segmento anteriore dorsale (manca l'arco ventrale) ed un segmento posteriore, di cui l'arco ventrale è formato dalla lamina sottogenitale.

Le femmine hanno sei segmenti addominali compiuti, e un primo segmento anteriore dorsale (manca l'arco ventrale). Il settimo segmento ha pure solamente la parte dorsale, nell'ottavo la vulva tiene il posto dell'arco ventrale; l'ultimo segmento poi costituisce la piastra sopraanale. Le ali anteriori hanno l'aspetto di elitre e s'incrociano longitudinalmente sul dorso quando sono ripiegate. Le ali possono mancare totalmente come nel genere Polyzosteria, oppure mancare solo nella femmina (Heterogamia, ecc.), oppure finalmente essere al tutto rudimentali (femmine del genere Blatta). Le zampe sono bene sviluppate, tutte di egual lunghezza e per lo più ricoperte di peli. Rispetto poi agli articoli dei tarsi noterò che vi hanno Blattidi eteromeri come nei Coleotteri. Il corpo è per lo più nudo e raramente coperto di peli. I colori non sono vivaci e variano dal grigio al bruno nero. Vivono quasi tutti i Blattidi, meno i generi Ectobia, Phyllodromia, Phoraspis, ecc., fuori del contatto della luce. Alcuni vivono nelle abitazioni dell'uomo, gli altri nelle foreste sopra varie specie di vegetali, sotto le pietre, le foglie secche ed anche nel legno marcio. Si nutrono per lo più di sostanze animali; non sdegnano tuttavia, soprattutto le specie domestiche, anche le sostanze vegetali. Sono voracissimi. 
I Blattidi sono sparsi per tutta la terra, qualche genere (Ectobia, Blatta, Periplaneta, ecc.) è cosmopolita. L'uomo ha contribuito e contribuisce tuttora moltissimo alla diffusione di varie specie di questo gruppo di Ortotteri. Poco studiata fino ad ora è la distribuzione geografica delle specie che vivono nelle foreste. I Blattidi finalmente sono caratterizzati dal fatto del deporre le uova rinchiuse in un involucro comune od ooteca più o meno allungata, contenente due serie regolari di ova in numero variabile da dieci a venti per ciascuna serie. Nelle specie che vivono nelle foreste vi ha, a quanto pare, un solo accoppiamento in giugno od in luglio. Nelle specie dome-

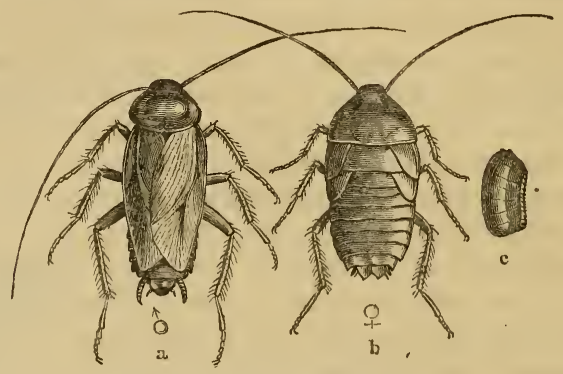

Fig. 44. Periplaneta orientalis.

$a$ maschio $-b$ femmina $-c$ ooteca.

stiche gli accoppiamenti si ripetono molto probabilmente varie volte nell'anno.

Varie specie di Blattidi tornano, sviluppandosi in grande numero, molto dannose all'uomo, il quale tuttavia può, tenendo bene pulite le sue abitazioni, facilmente distruggerle.

Ricorderemo fra i Blattidi i generi seguenti :

Ectobia Westwood. Ali ben sviluppate, coscie con piccole spine. Le specie di questo genere sono piccole, hanno colori oscuri, i quali variano qualche volta nei due sessi ( $E$. Lapponica) ed abitano i cespugli e le erbe di quasi tutta l'Europa. La $E$. Lapponica vive allo stato domestico in Lapponia, ove, secondo quello che riferisce Linneo, si nutre di pesce salato.

Blatta Linn. Antenne filiformi e più lunghe del corpo: ali 
ben sviluppate e di eguale lunghezza nei due sessi: zampe spinose: corpo nel maschio allungato, nella femmina invece dilatato. Questo genere, ricco di oltre cinquanta specie, contiene le specie più noceroli all'uomo di tutti i Blattidi. La B. germanica della lunghezza di circa 13 millimetri e che vive nei cespugli di quasi tutta l'Europa, è divenuta domestica soprattutto nell'Europa del Nord, dalla quale località pare abbia cacciato l'E. lapponica confinandola alla Lapponia. Oggi tuttavia pare che essa stessa venga sopraffatta dalla Periplaneta orientalis, specie più robusta. La $B$. germanica è divenuta cosmopolita.

Il genere Phoraspis Aud-Serv. merita di essere menzionato pei suoi colori brillanti, caso raro nei Blattidi; vivono le specie di questo genere principalmente nelle piante di mais o di zucchero dell'America centrale e meridionale.

La mancanza delle ali e le elitre rudimentali distinguono il genere Polyzosteria Burm., che comprende specie dell'Australia, delle Molucche, della Nuova Zelanda e del Messico.

Periplaneta Burm. Corpo oblungo nel maschio, allargato nelle femmine, elitre o bene sviluppate o rudimentali, ali o lunghe come le elitre, o di varia lunghezza, o mancanti al tutto e in generale più lunghe del corpo nel maschio, più corte nelle femmine; zampe spinose.

Conosciuta da tutti è la specie tipica di questo genere, la $P$. orientalis. L'ooteca di questa specie è di color bruno cupo, lunga circa 12 millimetri, e spessa 5. Secondo certi autori, questa specie cambierebbe sette volte la pelle prima di raggiungere lo stadio d' insetto perfetto: vive nelle nostre case, dove, a quanto pare, ha cominciato a diffondersi un duecento anni fa, importata dall'Oriente. Oggigiorno è sparsa per tutto il mondo.

Dannosissima pure è la $P$. americana, lunga una trentina di millimetri ed originaria dell'America meridionale. Un'altra specie, la $P$. Australasiae Fabr., tende pure oggi a farsi cosmopolita.

Il genere Corydia Aud-Serv. delle Indie Orientali, va ricordato pei colori vivaci ed eleganti delle sue specie.

Nel genere Polyphaga Brulè, le femmine ora hanno ali, ora ne sono prive. Nella $P$. aegyptiaca la femmina è attera. Questa specie si trova sulle spiagge mediterranee in Egitto, in 
Russia, in Persia, ecc. Nel genere Homoogamia Burm. i due sessi hanno le ali egualmente sviluppate. La sola specie conosciuta (H. mexicana) è del Messico.

Il genere Blabera Aud-Serv. comprende le forme più grosse dei Blattidi. Le femmine misurano sei ed anche sette centimetri di lunghezza. Sono voracissime, e, a quanto si dice, la $B$. gigantea Stoll. rosica nella notte le unghie delle persone addormentate. Le specie di questo genere vivono nell'America centrale e nell'America meridionale.

Menzioneró in ultimo il genere Cryptocercus S. Scudder, in cui $\mathrm{i}$ due sessi sono atteri ed in cui non vi hanno nell'addome che sette segmenti dorsali apparenti e sei ventrali. Fino ad ora se ne conosce una sola specie, il C. pustulatus degli Stati Uniti.

\section{MANTIDI.}

Gl'insetti di questa famiglia sono fra tutti gli Ortotteri i più schiettamente carnivori e predatori, ed il loro corpo presenta un piano di struttura che è in stretto rapporto colla loro indole. Il corpo è per lo piú allungato, il capo è ben sviluppato e ben distinto dal corpo: per la forma del capo i Mantidi hanno qualche affinità con molte forme di Pseudo-nevrotteri. Gli occhi sono ben sviluppati. Presentano inoltre i Mantidi tre ocelli più o meno sviluppati; le antenne sono filiformi e più corte del corpo nelle femmine, più lunghe invece nei maschi. In qualche maschio anzi (generi Blepharis, Empusa, ecc.) sono pettinate. Le mandibole sono molto robuste e sviluppate. Le zampe anteriori sono lunghe e foggiate ad organi di presa. Le medie e le posteriori sono per lo più lunghe e sottili, ora semplici, ora portanti delle espansioni laterali membranose, come ad esempio nel genere Empusa.

Il torace è per lo più relativamente molto lungo e stretto. Qualche volta, come nella Mantis mendica, presenta delle espansioni laterali.

L'addome è allungato, più tuttavia nei maschi che non nelle femmine, in cui anzi per lo più si presenta posteriormente dilatato. Nel maschio si contano otto segmenti ventrali, sei invece nella femmina. Le ali sono ben sviluppate, membranose ed hanno, per ciò che è della disposizione delle nervature, molta affinità coi Blattidi. 
I Mantidi hanno mole mediocre, non raggiungono mai le dimensioni di molti Fasmidi e neppure presentano forme molto piccole.

I colori dei Mantidi sono vari e per lo più chiari; predomina in generale il verde. Si trovano specie tuttavia elegantemente colorite, come si puó vedere dal lavoro di Saussure (Mission scientifique au Mexique et dans l'Afrique centrale. Parigi, 1872). I Mantidi presentano in un modo evidentissimo la corrispondenza dei colori del corpo col colore del mezzo, in cui essi vivono.

I costumi dei Mantidi sono notevoli per molti rispetti. Immobili sui rami di un cespuglio essi aspettano al varco la preda (mosche od altri Ditteri, Coleotteri, ecc., ed anche altri Ortotteri), di cui s'impadroniscono con un movimento rapidissimo delle estremità anteriori. Qualche specie dell'America del Nord, secondo lo Zimmermam, s'impadronirebbe anche di piccole rane. La Coptoteryx argentina poi di Buenos-Ayres, secondo quanto osservo il Burmeister, farebbe sua preda anche di piccoli uccelli.

Il modo di stare delle Mantidi colle lunghe zampe anteriori ripiegate quasi fossero in ginocchio, il protendere ora insieme, ora separatamente le stesse zampe anteriori, quasi volessero pregare, o chiamare l'elemosina, dà ragione dei nomi Mantis religiosa, M. oratoria, $M$. mendica, Empusa pauperata, ecc., che s'incontrano frequentemente nelle specie di questa famiglia. Le femmine delle Mantidi depongono generalmente (1) in Europa le uova in settembre od in ottobre circondandole di una ooteca, la quale, si noti, non esce già formata dell'ovidotto, ma viene fatta dall'animale durante la deposizione delle uova stesse. Le uova vengono, per mezzo di una sostanza viscosa preparata dalla femmina, fissate ai rami dei cespugli o sulle pietre.

Le larve, che sbucciano fuori (per lo più in giugno) dalle uova, hanno in complesso la struttura degli adulti; ma non fanno ancora uso delle zampe anteriori come organi di presa.

(1) Intorno al tempo della deposizione delle uova, ed al numero delle deposizioni annuali, non abbiamo ancora cognizioni precise, essendo esse strettamente legate al clima e variando secondo le regioni. 
A quanto pare, esse cambiano sette volte la pelle prima di giungere allo stato perfetto, al quale stadio pervengono, almeno nel contorno di Torino, verso la fine dell'estate od in principio dell'autunno.

Le Mantidi finalmente abbondano soprattutto nei paesi caldi, e più nel vecchio continente che non nel nuovo. Non salgono molto alto sui monti, in molti luoghi non oltrepassano i mille metri sul livello del mare e non si spingono molto verso il Polo. In generale non oltrepassano l'Europa centrale.

Menzioneremo i generi seguenti:

Eremiaphila: il corpo è corto, le ali sono rudimentali. Le specie di questo genere vivono nei deserti e abbondano nel continente africano. Secondo il Lefebre (1), esse potrebbero cambiare colore a seconda del colore terreno, sul quale camminano. La cosa meriterebbe di essere accertata meglio.

Le specie del genere Metalleutica sono da notarsi pei loro colori eleganti e metallici e per l'aspetto generale del loro corpo, che è quello delle Cicindele fra i Coleotteri. Sono state trovate specie di questo genere a Java e sulle coste del Malabar.

Mantis Linn. Questo genere è uno dei più diffusi fra quelli delle Mantidi. Vi si trovano piccole differenze fra i sessi: le antenne sono filiformi e non pettinate: le ali sono ben sviluppate: il protorace è allungato ed alquanto convesso. Si conoscono molte specie di Mantis, la più nota è la $M$. religiosa Linn., di un bel colore verde più o meno scuro, e qualche volta anche di un color giallo o rossiccio. Questa specie è comune in tutta l'Italia. Nel contorno di Torino è soprattutto abbondante sul monte Musiné, sul quale essa presenta molte varietà di colore.

Il genere Ameles Linn. comprende parecchie piccole specie, che abitano il bacino del Mediterraneo, l'Asia settentrionale, l'Africa meridionale, il Messico e l'Australia. Le ali o sono ben sviluppate o sono al tutto rudimentali, come ad esempio nella $A$. brevipennis Yersin della Francia meridionale.

(1) "Monogr. du genre Erémiaphile», Ann. Soc. Ent. de France, 1 serie, pag. 449 , tav. $11,12,13$. 
Fischeria Sauss. Troviamo in questo genere, ricco di specie proprie delle Indie e di varie regioni dell'Africa, le forme più grosse dei Mantidi. La $F$. Gigas misura in lunghezza 140 o 145 millimetri.

I generi Zoolea ·Aud-Serv., Theoclytes Aud-Serv., Blepharis Aud-Serv., Empusa Illiger, vanno distinti per avere le antenne del maschio pettinate. Le specie poi del genere Empusa devono essere ricordate per la presenza di espansioni membranose più o meno sviluppate sulle estremità posteriori. Le Empuse abitano l'Europa meridionale, l'Africa e l'Asia. La E. pauperata Rossi, che è tipo del genere, ha costumi simili in tutto a quelli della Mantis religiosa.

\section{FASMIDI.}

I Fasmidi hanno una certa affinità coi Mantidi e per molto tempo vennero ad essi riuniti. Se ne differenziano tuttavia per molti ed importanti caratteri.

Il corpo è allungato bacillare (genere Bacillus) ed in qualche altro caso invece appiattito (genere Phyllium). Il capo è mediocremente sviluppato: le antenne sono filiformi, e in qualche caso più lunghe del corpo. Il torace è allungato o stretto, l'addome ora è cilindrico ed allungato, ora invece è appiattito: spesso porta delle appendici laterali membranose; conta dieci anelli nei maschi. Le elitre sono poco sviluppate; le ali mancano o nella femmina soltanto $o$ in tutti due $i$ sessi. Le zampe sono lunghe e sottili; le anteriori sono in generale più lunghe delle altre. I tarsi sono in numero di cinque, il quinto ha inferiormente un bitorzolo spugnoso. Poco si sa intorno all'anatomia dei Fasmidi, poche pure sono le nostre cognizioni intorno al loro sviluppo. Il signor Murray, che ha studiato lo sviluppo del Phyllium seythe, ha osservato tre mutamenti della pelle.

I Fasmidi al contrario dei Mantidi sono insetti timidi e non aggressivi, vivono nei cespugli (fa eccezione il genere Prisopus, che ha vita in parte acquatica) e si nutrono di sostanze vegetali e soprattutto delle giovani gemme delle piante. Talvolta questi insetti sviluppandosi in gran numero arrecano danni rilevanti alla vegetazione. I Fasmidi presentano poi la proprietà notevole di riprodurre certe parti (soprattutto le zampe) che vengono loro mozzate. Questa proprietà è in rapporto 
molto probabilmente colla facilità di perdere le parti stesse (1).

I Fasmidi, come ho già avuto occasione di notare, presentano fatti molto spiccati di mimismo, e ciò è pure in rapporto colla loro natura timida, coll'assenza di armi speciali di offesa e di difesa. Il mimismo in questo caso è arma efficacissima di difesa.

I Fasmidi si trovano nell'antico e nel nuovo continente: abbondano nelle regioni equatoriali. L'Europa ne ha una sola specie. Poche pure sono le specie africane; molte invece quelle dell'America meridionale; molte più ancora quelle dell'Arcipelago indiano.

Sono da ricordarsi i generi seguenti:

Bacillus Latr. Corpo allungato, cilindrico: antenne corte: organi del volo mancanti al tutto. Delle quaranta e più specie di questo genere una sola il $B$. Rossi si trova in Europa ed in Italia.

Affine al genere Bacillus è il genere Bacteria quasi esclusivamente americano. Una specie di grande mole, la $B$. arumatia Stoll di Caienna, lunga m. 0,40, è priva di ali nei due sessi ed ha antenne lunghe e filiformi.

Atteri sono pure i generi Anisomorpha Gray (proprio dell'America meridionale); Rhaphiderus Aud-Serv. (questo genere conta numerose specie che abitano molte regioni dei due mondi: Nuova Zelanda, .Nuova Olanda, Giappone, Ceylan, Isola di Francia, Brasile, ecc.); Euryacantha Boisduv (abita la regione austromalese).

Il genere Cyphocrana Aud-Serv., il quale presenta ali ben sviluppate nei maschi, meno sviluppate nelle femmine, comprende forme gigantesche proprie delle regioni equatoriali dell'antico continente.

Le specie del genere Phasma Ill. hanno ali ben sviluppate nei due sessi ed antenne lunghissime soprattutto nei maschi.

Il genere Prisopus Aud-Serv. merita di essere menzionato per la sua vita acquatica (2). Si trovano specie di questo genere nell'America meridionale e nell'Africa meridionale.

(1) Michele Lessona, Sulla riproduzione delle parti, Atti della Soc. Ital. di Sc. Nat., vol. XI, $18 \dot{6} 68$.

(2) Secondo il sig, Fry, che ha osservato i costumi del $P$. flabelliformis Stoll., durante il giomo i Prisopi stamno al fondo dell'acqua attaccati alle pietre ed ai corpi sommersi e non escono a volo che al erepuscolo. 
Noto a tutti per le sue forme strane è il genere Phyllium Illiger, il quale conta una quindicina di specie, che imitano le forme di varie sorta di foglie. Si trovano le specie di questo genere nell'Arcipelago indiano e nell'isole Maurizio.

ACRIDIDI.

Il corpo allungato e compresso lateralmente, e le zampe posteriori, ingrossate ed atte al salto, distinguono gli Ortotteri di questa famiglia dalle precedenti. Il capo ha forma conica per lo più e subglobosa, in qualche caso ha il vertice più o meno prolungato (genere Xiphocera, Truxalis, ecc.). Le antenne ora sono allungate e filiformi, ora appiattite e trigonali; la loro lunghezza non è mai molto grande e non supera la metà del corpo. Gli occhi sono ben sviluppati: vi hanno per lo più sulla fronte tre ocelli. L'apparato boccale è robusto e le mandibole sono molto forti.

Ben sviluppati sono in generale gli organi del volo; mancano tuttaria completamente nei generi: Proscopia e Batrachotettix; e sono poco sviluppate nei generi Porthetis, $\mathrm{Pe}$ zotettix, ecc. Le elitre sono opache e coriacee ed in generale presentano colori poco vivaci ed in rapporto col mezzo in cui vivono: le ali membranose, molto variabili fra loro in ampiezza, si mostrano per lo più elegantemente colorite. Questa colorazione è assai variabile anche negl'individui della stessa specie, quindi non ha molta importanza nella diagnosi delle specie. Le zampe anteriori e mediane sono sottili, e presentano per lo più delle numerose e forti spine: le posteriori (meno nel genere Pneumora, sono atte al salto. I tarsi contano tre articoli. L'addome ha nove (o dieci) segmenti. Dobbiamo notare ancora, che i maschi producono un suono speciale fregando la parte interna delle coscie posteriori, che è dentellata, contro le nervature delle elitre. Nella femmina questo apparecchio, destinato a produrre un suono determinato, è al tutto rudimentale.

Il canal digerente negli Acrididi, contrariamente a quanto si potrebbe supporre ponendo mente al regime vegetale di questi animali, è lungo quanto il corpo.

Gli Acrididi sono voracissimi e si nutrono di sostanze vegetali. La loro mole puó variare da tre o quattro millimetri, come in qualche Tettix, a cento settantacinque millimetri, come 
nella Proscopia gigantea Klug. Le uova vengono nell'Europa temperata deposte in autunno e non si sviluppano che nella successiva primavera. Pare tuttavia che alcune specie passino l'inverno e non depongano le uova che nella primavera susseguente. Questo fatto è in rapporto col clima.

Gli Acrididi sono sparsi per tutta la terra e vi hanno specie che abitano le regioni aride o secche, altre che amano le praterie umide, altre che vivono nei boschi, altre fra le roccie, ecc.

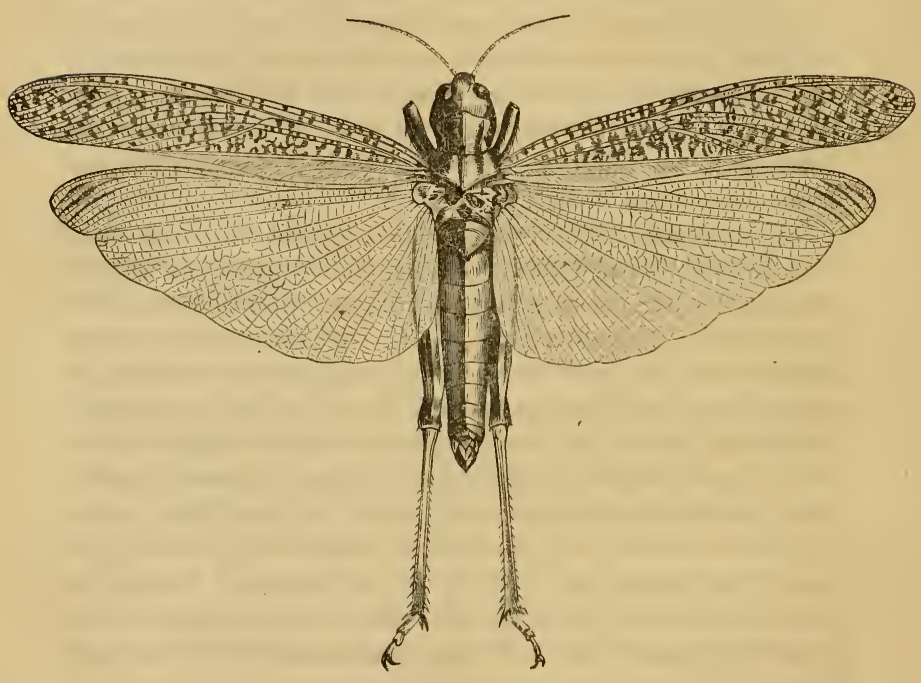

Fig. 45. Acridium migratorium.

Non vanno sulle Alpi in generale che ai duemila o duemila cinquecento metri sul livello del mare.

Hanno una grande tenacità di vita ed è a questo proposito da ricordarsi il caso osservato dal Zinnani di due Acrididi accoppiati, che, essendo stati tagliati trasversalmente nell'addome, continuarono tuttavia a saltellare per parecchi giorni.

Offrono anche una grande resistenza al freddo, come io stesso ho potuto ripetutamente osservare.

Il fatto più importante della vita degli Acrididi si è quello 
delle migrazioni ch'essi compiono in molte regioni della terra arrecando gravi danni all'uomo. Le specie che più frequentemente presentano il fatto delle migrazioni sono le seguenti :

$$
\begin{aligned}
& \text { Acridium peregrinum, } \\
& \text { "tartaricum, } \\
& \text { Pachytylus migratorius, } \\
& » \quad \text { cinerascens, } \\
& \gg \text { stridulus (raramente), } \\
& \text { Caloptenus italicus, } \\
& \text { Stauronotus cruciatus, } \\
& \text { Oedipoda fasciata (raramente). }
\end{aligned}
$$

Le migrazioni degli Acrididi non sono periodiche, non si fanno cioè a periodi fissi come quelle degli uccelli: la mancanza di nutrimento pare sia la causa prima delle migrazioni degli Acrididi.

Una grande quantità d'individui delle specie sopracitate abita le regioni aride dell'Africa settentrionale e le steppe dell'Asia centrale. Quando il cibo in queste località è sufficiente a nutrire tutti gli individui, questi non emigrano; quando invece sviluppandosi essi per una qualche causa in troppo grande numero, non trovano più sul luogo sufficiente cibo, si levano a volo e riuniti in sciami grandissimi vanno a cercare altri luoghi più propizii. Avviene spesso che un colpo di vento trasporti questi sciami molto lontano.

L'Europa meridionale è non raramente danneggiata da invasioni di Acrididi (1).

Tettix Fisch. Il carattere principale delle specie di questo genere si è il grande prolungamento posteriore del pronoto. Le elitre sono piccole e rimangono nascoste dal prolungamento del pronoto. Hanno piccola mole e sono sparsi ovunque nei giardini, sull'orlo delle foreste e delle strade, ecc. La specie più comune fra noi è la $T$. subulata di color grigio o bruno con due macchiette bianche ai lati del pronoto e con dei punticini neri.

Pneumora Thunberg. Il carattere più importante di questo

(1) Per maggiori particolari intorno alle migrazioni degli Acrididi, si consulti M. Girard, Les insectes. Traité élémentaire d'Entomologie, vol. II, Parigi 1876. 
genere, come io ho già detto, sta nelle zampe posteriori, che sono più corte dell'addome, non ingrossate e quindi non atte al salto. Le femmine sono attere. Le specie di questo genere abitano l'Africa australe.

Il genere Stenobothrus Fisch. (Gomphocercus, Burm.) comprende un grande numero di specie molto difficili da separare. le une dalle altre. Il corpo è allungato e di mediocre grandezza: il capo è leggermente conico: il vertice è alquanto sporgente con una piccola cavità da ciascun lato: le antenne sono mediocremente lunghe, subcilindriche e spesso alquanto compresse, e non appuntite. Il pronoto è alquanto piano ed ha tre carene. Lo sviluppo delle ali varia nelle varie specie. Le zampe sono sottili: le posteriori ingrossate ed atte al salto. L'addome inferiormente è carenato. L'apparato stridulatore è molto sviluppato.

Le specie del genere Stenobothrus vennero riunite in vari gruppi tenendo conto della forma delle antenne e del pronoto.

Lo $S$. pratorum è una delle specie che più frequentemente s'incontra in estate ed in autunno nei prati del nostro paese ed in generale di tutta l'Enropa. La sua colorazione variabilissima ha dato origine ad un grande numero di varietà. Varietà più numerose ancora che non nella specie precedente rendono difficilissimo lo studio di un'altra specie, pure assai comune, lo S. variabilis Fischer.

Per maggiori particolari intorno a questo genere si ricorra alla classica opera del Fischer intorno agli Ortotteri europei.

Generi affini al precedente sono i seguenti: Stetheophyma Fisch., e Epacromia Fisch., i quali hanno pure rappresentanti fra noi.

Le specie del genere Oedipoda Latr. vanno distinte in generale per la vivacità o per l'eleganza dei colori delle loro ali. Corpo allungato; capo relativamente largo e grosso; antenne filiformi, più lunghe nel maschio che non nella femmina; pronoto rugoso, colla carena mediana più o meno spiccata: ali ed elitre ben sviluppate; le elitre molto diverse dalle ali e di color grigio-bruno: le ali aventi colori vivaci, in cui predominano il rosso e l'azzurro. Vivono nei luoghi sabbiosi del piano e dei monti sui quali tuttavia non salgono molto in alto. Fra noi l'Oe. fasciata Siebold, è la specie più comune e 
presenta due varietà o due specie, secondo il valore che si dà ai caratteri differenziali. La prima varietà, l'Oe. coerulescens Linn., ha le ali azzurre fasciate verso l'apice di nero, l'altra, l'Oe. germanica Linn., ha invece le ali di un bel color rosso. In qualche luogo queste due varietà si trovano isolate l'una dall'altra; in altri invece le due varietà vivono insieme.

Affine al genere Oedipoda è il genere Pachytylus Fischer, il quale comprende specie che abitano l'Europa, l'Asia e l'Africa. Il $P$. migratorius Linn. è tristamente celebre per le sue emigrazioni.

Non dimentichiamo il genere Caloptenus, di cui una specie, il C. italicus, riesce in molti luoghi dannosa alla vegetazione, ed il genere Acridium, le di cui specie abitano principalmente l'Africa e l'America. L'A. lineola non raro fra noi è uno dei più grossi Ortotteri dei nostri paesi. Il maschio è lungo una cinquantina di millimetri, la femmina spesso più di sessanta. Varie specie di questo genere sono migratrici.

Truxalis Fabr. Corpo allungato e sottile; il capo è prolungato anteriormente in forma di cono; le antenne sono corte compresse, leggermente triedre, larghe alla base e strette verso l'apice: le elitre sono più lunghe in generale dell'addome: le ali sono ben sviluppate: le zampe sono lunghe e sottili. Le specie di questo genere si trovano quasi esclusivamente nelle regioni meridionali dell'antico continente. La specie più nota e più comune fra noi è la $T$. nasuta Linn., la femmina della quale puó misurare perfino una settantina di millimetri di lunghezza.

Ricordiamo in ultimo il genere Proscopia Klug., dell'America meridionale, il quale ha specie attere e dal corpo sottile, allungato, e che ricordano pel complesso dei caratteri e le Truxalis e i Phasma.

\section{Locustid.}

Il corpo è allungato ed alquanto compresso lateralmente. Il capo è grande, verticale o un po' sfuggente all'indietro, come ad esempio nel genere Conocephalus. Le antenne sono filiformi e lunghe come il corpo o più lunghe. Mancano gli ocelli. L'apparato boccale è robusto e ben sviluppato. Le ali e le elitre possono essere ben sviluppate od essere rudimentali, come si osserva in un buon numero di Locustidi, od 
anche mancare quasi affatto. Sono rudimentali ad esempio nei generi Decticus e Xiphidium: mancano quasi intieramente nel genere Ephippigera: mancano al tutto e in tutti due i sessi nel genere Raphidophora. Le zampe per lo più non sono molto grosse; le posteriori sono in generale più sviluppate delle altre e foggiate pel salto. I Locustidi tuttavia sono proporzionatamente meno buoni saltatori degli Acrididi. I tarsi contano tutti quattro articoli forniti inferiormente di una spazzoletta. La struttura degli organi interni mostra una grande affinità fra i Locustidi ed i Grillidi, affinità del resto che si scorge anche col semplice esame dei caratteri esterni. Il canal dirigente, quantunque il regime dei Locustidi sia esclusivamente vegetale, è corto e robusto, e misura al più una volta e mezzo la lunghezza del corpo. Ricorderemo ancora due apparati speciali, probabilmente uditivi, collocati sulla faccia interna ed anteriore del primo paio di zampe.

La femmina finalmente ha un' ovopositore per lo più relativamente molto lungo. L'apparato del suono è bene sviluppato. Le uova vengono deposte, nella maggior parte delle specie, nella terra e si sviluppano $o$ in sul finire dell'autunno o al principio della primavera successiva. Forse tre sono le mute che l'insetto subisce prima di giungere allo stato perfetto.

Non giungono a mole molto grande e presentano per lo più colori verdi. Vivono principalmente nei boschi e nei luoghi erbosi dell'Europa, dell'Asia e dell'America; sono scarsi invece nell'Africa, arida in generale e secca, nella quale abbondano al contrario gli Acrididi.

Meconema Aud-Serv. Corpo piccolo, ali ed elitre ben sviluppate in tutti due i sessi. Questo genere comprende una sola specie: il M. varium Fabr., non raro nell'Europa centrale e meridionale.

Molto più numerose che non nel genere precedente sono le specie del genere Phaneroptera Aud-Serv. Questo genere ha ali ben sviluppate, corpo allungato, sottile ed agile. Il genere Phaneroptera abita le regioni temperate e calde dei due emisferi. Fra noi sono comuni: la $P$. falcata Scop., e la $P$. liliifolia Fabr.

Pterocroza Aud-Serv. Phylloptera Aud-Serv. Le specie di questi generi quasi al tutto esclusivi dell'America meridionale 
meritano di essere notate per rassomiglianze che esse presentano con varie sorta di foglie.

Decticus. Elitre ed ali ora ben sviluppate ed atte al volo, ora rudimentali : mandibole grandi e forti : due spazzolette alla base dei primi articoli dei tarsi posteriori. Molto numerose sono le specie di questo genere ed abitano per lo più le regioni aride dei paesi temperati d'Europa, di Asia, d'Africa e d'America. Si trovano anche sui monti e si innalzano anche fin oltre ai duemila metri. La specie più nota e nello stesso tempo una delle più grosse del genere è il $D$. verrucivorus Linn., che abita tutta l'Europa (1).

Locusta Fabr. Corpo grande: antenne filiformi e più lunghe del corpo: ali ed elitre ben sviluppate e in generale più lunghe dell'addome : ovopositore lungo, dritto. La comunissima L. viridissima Linn., è il tipo di questo genere. Questa specie è

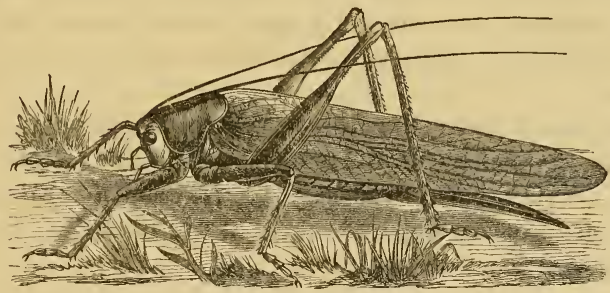

Fig. 46. Locusta viridissima.

di color verde-chiaro: ha le ali che misurano in lunghezza il doppio dell'addome: ha un apparato stridulante molto sviluppato e vive spesso in gran numero sugli alberi e nei prati. Molto probabilmente, come fa osservare il Girard (2), questa specie è anche carnivora (3).

Il genere Saga Charp. merita di essere ricordato per la

(1) Venne dato il nome di verrucivorus a questa specie dal costume dei contadini Svedesi di far mordere da questo insetto le verruche della pelle credendo che il liquido nerastro cacciato fuori dalla bocca di questo Ortottero abbia la proprietà di far scomparire le verruche stesse.

(2) Op. citata.

(3) Questo fatto si verifica pure come ho fatto osservare nelle Forficole. 
sua rassomiglianza con certi Fasmidi e poi perchè comprende i giganti fra i Locustidi europei. La S. serrata, femmina, può misurare perfino settanta millimetri di lunghezza.

Le specie del genere Callinemus, Pycnogaster, Onconotus, ecc., hanno corpo grosso e pesante, elitre rudimentali ed ali mancanti e vivono nell'Europa meridionale ed orientale (1).

Ephippigera Aud-Serv. Corpo non molto lungo e tozzo: antenne filiformi e più lunghe del corpo: elitre squamiformi, corte, incurvate e per lo piu fortemente reticolate e rugose: apparato stridente in tutti due i sessi. Le Efippigere sono numerose; in ispecie abitano l'Europa meridionale ed il nord dell'Africa. Comune fra noi nel mese di agosto e di settembre nelle vigne è l'E. vitium.

Affine per molti caratteri al genere Ephippigera è il genere Odontura: l'O. serricauda di color verde macchiato di nero e con striscie gialle sul capo, sul torace e con l'orlo dell'elitre giallo si trova, non mai a dir vero, abbondante in varie regioni dell'Europa centrale e meridionale: non è rara in Piemonte.

Una speciale menzione merita il genere Raphidophora AudServ. Questo genere fa il passaggio ai Grillidi. Molte delle sue specie vivono nelle caverne. La $R$. cavicola Kollard, si trova nelle caverne della Carniola e dell'tlliria ed anche a Siracusa.

Il genere Anostostoma Gray, finalmente, presenta di notevole il grande sviluppo delle mandibole. Tre sono le specie appartenenti a questo genere conosciute fino ad ora, una di Australia, l'altra del Surinam ed un'altra del gruppo d' isole del Madagascar. Notevole pure è il costume di queste specie di scavare nei rami degli alberi delle gallerie nelle quali esse vivono.

GRILLIDI.

Gli Ortotteri di questa divisione si distinguono dagli altri per avere corpo tozzo e cilindrico. Il capo è grosso, le antenne

(1) In Italia il genere Onconotus, proprio della Russia meridionale, è rappresentato dal genere affine Anonconotus (L. Camerano "Descrizione di un nuovo genere e di una nuova specie di Ortottero piemontese », Atti della $R$. Accad. delle Scienze di Torino, vol. XIII, 1878). 
sono in generale lunghe e filiformi. Gli ocelli sono per lo più in numero di due o tre. Le mandibole sono molto robuste.

Variabile è lo sviluppo delle ali; esse mancano intieramente nei generi Myrmecophila e Mogoplistes.

Le zampe anteriori sono in qualche genere, Grillotalpa, Tridactylus, ecc., foggiate per scavare. Le posteriori sono più o meno ingrossate ed atte al salto. I tarsi hanno tre articoli. L'addome conta nove o dieci segmenti dorsali ed otto ventrali. I maschi hanno in generale un apparato assai sviluppato, destinato alla produzione di un suono. A questo proposito ricorderò la presenza in molte specie di Grillidi di organi musicali virtuali, come ha dimostrato il Landois (1) nella comune Grillotalpa e nel comune grillo dei campi.

La famiglia dei Grillidi comprende un numero di forme minore che non le famiglie precedenti. Essi sono sparsi per tutta la terra, mancano tuttavia nelle alte regioni alpine e nelle regioni più settentrionali. Non si trovano ad esempio, a quanto pare, in Lapponia.

La maggior parte delle specie di Grillidi abita nelle buche del suolo, alcune vivono colle formiche, altre nelle case stesse dell'uomo (Grillus domesticus), altre infine, ma in piccol numero, fra le erbe ed i cespugli. Sono omnivori.

I colori dei Grillidi sono poco appariscenti e poco variati; predominano il nero ed il bruno. In qualche caso vi hanno delle macchie di un rosso vivissimo.

Ricordiamo i generi seguenti :

Gryllotalpa Latr. Questo genere è facilmente riconoscibile dallo sviluppo delle zampe anteriori atte a scavare, dal corpo cilindrico e quasi intieramente ricoperto da leggiera lanuggine e da un complesso di forme che ricordano il corpo della talpa ed indicano un animale eminentemente scavatore. Gli occhi sono piccoli : vi hanno due ocelli. Nella femmina non vi è ovopositore. La specie più nota di questo genere è la G. vulgaris Latr., la quale abita per lo più gli orti, i giardini ed i campi di grano, arrecando, quando si sviluppa in grande

(1) : Ueber ein dem sogenannten Tonapparat der Cicaden analoges Organ bei den hiesigen Gryllen ». Zeit. f. Zool., vol. XXII, 1872. 
numero, non lievi danni alle piante coltivate colle numerosissime e lunghe gallerie che essa scava e nelle quali passa la sua vita. La comune Grillotalpa è ad un tempo carnivora e fitofaga; preferisce, a quanto pare, gl'insetti, in cerca dei quali spesso scava lunghe gallerie. Una quindicina o poco più sono le specie del genere Gryllotalpa e sono sparse per tutte le parti del mondo (1).

Il genere Myrmecophila Latr. è notevole per la sua forma e per i suoi costumi. Manca di tutte due le paia di ali ed ha in complesso l'aspetto di una piccola larva di una blatta e non giunge che a cinque millimetri di lunghezza. Le specie di questo genere vivono insieme con varie specie di formiche $(F$.glebaria, fusca, rufa, sanguinea, ecc.), delle quali si possono considerare siccome commensali. Intorno ai curiosissimi costumi delle specie del genere Myrmecophila si consulti: Savi, Os-

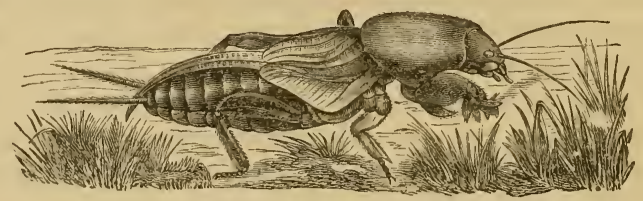

Fig. 47. Gryllolalpa vulgaris.

servazioni sopra la Blatta acervorum, Panzer. Pisa 1820.

Le specie appartenenti al genere citato sono state trovate in varii paesi d'Europa e d'Africa: in Italia, in Francia, in Germania, in Russia, in Algeria, ecc., ma sono rare ovunque.

Oecanthus. Il corpo è depresso, allungato; le ali sono molto sviluppate; le zampe pure sono relativamente lunghe: le specie di questo genere sono distinte pei loro colori chiari e per lo sviluppatissimo apparato stridente, di cui l'animale fa uso principalmente di notte. La specie più nota e più diffusa è l' $O$. pellucens Scop., di color grigio-giallastro-pallido, con macchiette e striature brune.

(1) Molti sono i mezzi che vennero proposti per distrurre le grillotalpe; io non posso entrare a trattare diffusamente questo argomento. Si consulti a questo proposito oltre ai libri citati al capo II anche lo scritto seguente: Michele Lessona "Le rufole in Torino ", Ann. della R. Accad. d'Agricolt., vol. XIX, 1876. 
Gryllus. Il corpo è tozzo e cilindrico; il capo è sferoidale; le antenne sono filiformi ed in generale più lunghe del corpo. Le ali sono variamente sviluppate secondo le specie e secondo i sessi. Le tibie delle zampe posteriori portano due file di spine. Le specie di questo genere abitano le regioni calde e non si estendono in Europa al di là del cinquantesimo parallelo. Il $G$. domesticus vive nelle nostre case; il G. campestris e le altre specie affini vivono invece in piccole buche del terreno. Gli uni e gli altri poi sono noti pel sonoro cri-cri che essi

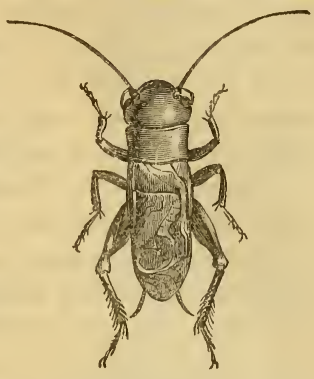

Fig. 48. Gryllus campestris. fanno sentire nelle notti della buona stagione.

Le specie del genere Gryllus sono notevoli anche per la presenza di uno spermatoforo nei maschi.

\section{Pseudoneorotteri.}

$A$. Larve che non hanno vita acquatica.

b. Mandibole sottili e deboli. Apparato boccale foggiato per succiare

Fisopodi.

$b^{\prime}$. Mandibole robuste. Apparato boccale destinato a masticare.

Corrodenti. B. Larve che fanno vita acquatica ed hanno branchie. Anfibiotici.

I Fisopodi, che comprendono una sola famiglia, quelle dei Tripsidi, hanno forme che non si sa bene ancora dove debbano essere collocate e che presentano certe affinità coi Rincoti, coi quali rimasero uniti per qualche tempo, e cogli Ortotteri. Qualche autore ha fatto dei Thrips un ordine speciale.

I Tripsidi sono insetti di piccolissima mole (lung. m. 0,002 e 0,003), i quali hanno ali membranose quasi al tutto prive di nervature ed orlate di lunghi peli, i tarsi di due articoli e terminati da una ventosa. Vi sono tre ocelli. Le antenne sono filiformi. Vivono le specie di questa famiglia sui fiori, sulle foglie e sotto la scorza degli alberi.

I cereali e soprattutto il grano sono talvolta seriamente danneggiati dal Thrips cerealium. Affini al genere Thrips sono i generi Heliothrips, Melanothrips, ecc., fondati dall'Haliday. 


\section{Corrodents.}

Molto varie fra loro sono le forme che entrano a costituire questo gruppo di insetti; riesce quindi difficile di poter dare dei caratteri generali. Caratteri tuttavia comuni a tutte le forme di corrodenti sono la presenza di un piccolo numero di nervature sulle ali e un apparato boccale relativamente forte, robusto e destinato a masticare.

La mole varia dai Psocidi piccolissimi alle Termiti; le ali ora sono ben sviluppate, ora mancano al tutto. Vario pure è il nutrimento, il quale è o di sostanze vegetali per lo più essiccate o di sostanze animali.

Gli uni, come le Termiti, sono sociali, e presentano fenomeni di polimorfismo che sono una conseguenza della vita sociale: gli altri come i Psocidi e gli Embidi non sono sociali.

I Corrodenti sono sparsi per tutta la terra, abbondano tuttavia principalmente verso l'equatore, e qualche specie (Termiti), arreca danni gravissimi all'uomo.

Fra i Psocidi distinti per avere la fronte vescicolosa, le antenne lunghe e filiformi e per la mancanza in qualche caso delle ali, menzioneremo il genere Troctes Burm. in cui mancano le ali, ed i tarsi hanno tre soli articoli. Il T. pulsatorius Fabr. (1) abbonda principalmente nelle collezioni di Storia naturale e soprattutto nelle entomologiche (alle quali quando si sviluppa in troppo grande quantità riesce dannosissimo, rosicchiando le parti interne del corpo degli insetti e facendo si che questi cadano sfasciati), nelle biblioteche e luoghi analoghi.

Il genere Psocus Lat. è caratterizzato dalla presenza di ali variamente sviluppate, e in qualche caso presentanti numerose squamette, dalla presenza di tre ocelli e dal tarso con due soli articoli. Vivono le specie di questo genere sui tronchi di varie sorta di alberi. Molte sono le specie conosciute e queste sono sparse per tutte le regioni della terra. Se ne conoscono varie specie fossili nell'ambra.

(1) Si credette per molto tempo che questo animaletto dall'integumento molle producesse quei colpi secchi come $\mathrm{i}$ battiti di un orologio che si sogliono udire nei legni tarlati, colpi che, come vedremo, sono prodotti invece da un Coleottero del genere Anobium. Questa credenza fece dare al primo il nome volgare di orologio della morte ed il nome scientifico di $P$. pulsatorius, ecc. 
Gli Embidi comprendono forme poco note e che hanno molta affinità colle Termiti: le ali sono ben sviluppate, le antenne sono filiformi, il numero dei loro articoli varia da dodici a trentadue. Gli Embidi abitano le regioni tropicali. Se ne conosce una specie fossile.

Notevoli pei loro costumi e per la loro vita sociale sono le Termiti o formiche bianche, caratterizzate dall'avere antenne filiformi, di diciotto o venti articoli, ali per lo più molto sviluppate e tarsi di quattro articoli. Le Termiti ci presentano un fatto di polimorfismo che è una conseguenza della loro vita sociale. Le forme principali che costituiscono le società delle Termiti sono le seguenti (1):

Individui provvisti di ali $\left\{\begin{array}{l}\text { maschio fecondo } \\ \text { femmina feconda }\end{array}\right.$ Individui privi di ali $\left\{\begin{array}{l}\text { larve } \\ \text { ninfe } \\ \text { neutri }\end{array}\left\{\begin{array}{c}\text { soldati } \\ \text { operai. }\end{array}\right.\right.$

La femmina perde poco dopo l'accoppiamento le ali, ed il suo addome ingrossa straordinariamente riempiendosi di uova.

Le termiti costruiscono ora dei grossi e saldi cumuli di terra (alti da 3 metri a 3,70), come si osserva nel $T$. bellicosus dell'Africa meridionale, ora dei nidi sferoidali sugli alberi come è stato osservato in specie Australiane.

Per quanto se ne sa oggi, le Termiti avrebbero nei loro costumi molta affinità colle formiche (2).

(1) Le nostre cognizioni in proposito sono ancora molto scarse ed incerte. L'Hagen che studiò diligentemente questi insetti avrebbe in una società di Termiti riconosciuto le forme seguenti: $1^{\circ} \mathrm{Un}$ re fecondo e una regina piena di uova; $2^{\circ}$ Individui adulti alati maschi e femmine; $3^{\circ}$ Ninfe dei due sessi provviste di grandi astucei delle ali; $4^{\circ}$ Ninfe pure dei due sessi provviste di piccoli astucci delle ali; $5^{\circ}$ Larve maschi e femmine; $6^{\circ}$ Operai atteri; $7^{\circ}$ Larve di operai; $8^{\circ}$ Operai di forma speciale, col capo ciò̀ provvisto di prolungamento anteriore a mo' di un becco; $9^{\circ}$ Soldati atteri; $10^{\circ}$ Larve di soldati; $11^{\circ}$ Soldati di forma speciale simili agli operai del numero $8^{\circ} ; 12^{\circ}$ Soldati, forse anormali, con astucci delle ali.

(2) Si consulti per maggiori particolari oltre l'opera capitale di H. A. HAGEN, Monographie der Termiten, in Linnaea entomologica, volumi X, XII, XIV, anni 1855-1858-1860, anche il trattato d'Entomologia del Girard. Parigi 1876. 
Si conoscono molte forme fossili di Termiti nell'ambra. Le Termiti abbondano nelle regioni tropicali; poche sono le specie attualmente viventi in Europa, e forse vi sono state importate (1).

Straordinaria è la voracità di questi insetti; nessuna sostanza loro resiste, ed anche i legni più duri delle regioni equatoriali vengono da essi intaccati e rosicchiati. Riescono le Termiti dannosissime all'uomo, scavando in modo che l'uomo non se ne accorge, dall'interno cioè verso l'esterno, che non intaccano mai, con innumerevoli gallerie, i legnami che entrano

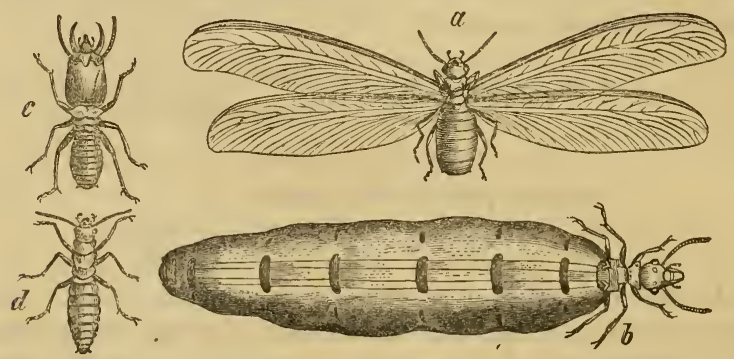

Fig. 49. Termes bellicosus.

$a$ maschio $-b$ femmina colle uova $-c$ guerriero $-a$ operaio.

nelle costruzioni delle case, e cagionando cosi molte volte la rovina delle case stesse.

Le Termiti hanno molti nemici. Primo di tutti è l'uomo, il quale in molte località, come ho già avuto opportunità di notare, le mangia. Si nutrono pure di Termiti fra i mammiferi molti Sdentati, certi Didelfi e l'Echidna.

\section{ANFibiotici.}

Il carattere più importante di questo gruppo di Pseudonevrotteri si è quello di avere nello stato larvale una vita acquatica e di essere provvisti di branchie, organi che in qualche caso si conservano più o meno sviluppati anche nello stato adulto (2). Probabilmente, come ho già detto, gli Anfibiotici

(1) Calotermes flavicollis Fabr. Termes lucifugus Rossi. T. flavipes Kollar.

(2) Pteronarcys regalis New. del Canadà, cce. Vedi Gerstaecker « Zur Mor- 
sono i più antichi di tutti gli insetti : sono il ceppo da cui tutti gli insetti sono derivati.

A. Ali di ineguale lunghezza: le anteriori più lunghe delle posteriori.

a. Differenza fra le due paia di ali non molto grande: tarsi con tre articoli . . . . . . . . Perlaridi.

b. Ali anteriori molto più grandi delle posteriori: tarsi con quattro o cinque articoli . . . . . . Efimeridi.

$B$. Ali di eguale lunghezza e fittamente reticolate . Libellulidi.

\section{PERLARIDI.}

Questa famiglia è una delle più naturali degli Ortotteri, e presenta una qualche affinità colle Friganee colle quali venne per molto tempo riunita dagli $\mathrm{Au}$ tori. Differisce da queste principalmente per non avere metamorfosi compiute.

La famiglia dei Perlaridi comprende insetti di piccola mole, dal corpo depresso ed allungato e dall' integumento mediocremente duro e quasi sempre ricoperto di piccolissimi peli.

Gli ocelli sono in nu-

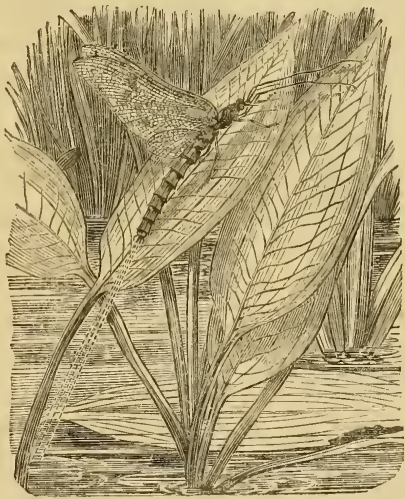

Fig. 50. Ephemera vulgata. mero di tre, le antenne sono allungate e filiformi, l'apparato boccale è poco sviluppato negli adulti.

Le ali sono ineguali, le inferiori sono quasi sempre più grandi alla base delle superiori; le ali posteriori possono in parte ripiegarsi verso il basso. Qualche volta le ali sono atrofizzate nei maschi.

L'ultimo segmento addominale porta quasi sempre due lunghi filamenti.

phologie der Orthoptera amphibiotica, ecc., e Ueber das Vorhommen von Tracheen Kiemen bei ausgebil. Insehten. Zeit. f. Zool., vol. XXIV, 1874. 
Le larve e le ninfe fanno vita nell'acqua e sono carnivore (si nutrono di piccoli insetti acquaiuoli e specialmente di larve di Efimere, di Agrion, ecc.), e, cosa molto importante, hanno delle trachee branchiali che loro servono per respirare l'aria sciolta nell'acqua. Le branchie esterne mancano tuttavia nelle larve di qualche specie del genere Nemura. La respirazione in questo caso o è intestinale o semplicemente integumentale. Nel genere Pteronarcys americano, come ho già fatto notare, e, secondo il Mac Lachlan, anche in varie specie europee persistono nello stato adulto vari filamenti branchiali collocati ai lati del corpo.

I Perlaridi vivono sempre in prossimità delle acque. Nello

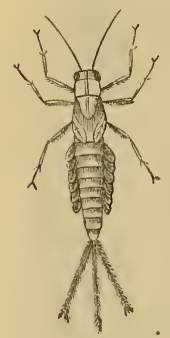

Fig. 51. Larva di Efimera. stato adulto, stato che dura poco non vivendo che pochi giorni dopo la metamorfosi, non si nutrono (1); gli organi boccali sono perciò allo stato rudimentale.

I colori di questi insetti sono poco appariscenti, variano dal nero, dal bruno al grigio ed al giallognolo: sono sparsi per tutta la terra, ma fino ad ora non vennero studiate un po' diligentemente che le specie europee. Si innalzano sulle Alpi fino a duemila cinquecento metri circa e si spingono verso il nord fino nell'estrema Lapponia.

Fra le specie di Perlaridi Europee ricorderò la Perla bipunctata Pictet, lunga una trentina di millimetri o poco più, dal capo e dal corpo macchiato di giallo e di bruno-nero e dalle setole (la presenza delle setole è carattere comune delle specie del genere Perla) dell'ultimo segmento addominale ben sviluppate, e la Nemura variegata Oliv., priva delle setole addominali (carattere anche qui generale delle specie del genere Nemura). Il colore della $N$. variegata è bruno, il primo articolo delle antenne è giallognolo, le parti inferiori dell'addome sono nerastre e le ali sono grigio-brune alquanto opache.

EFIMERIDI.

Gli Efimeridi hanno molti punti di contatto e coi Perlaridi

(1) Farebbe eccezione il genere Pteronarcys che ha organi boccali ben sviluppati, e che sarebbe, a quanto pare, carnivoro nello stato adulto. 
e coi Libellulidi; si distinguono tuttavia facilmente per la diversità di sviluppo delle ali anteriori e posteriori; queste ultime sono molto più piccole delle prime. Le antenne sono molto corte (in qualche caso mancano al tutto come in qualche specie del genere Chöleon Leach.), ed hanno tre articoli: gli organi boccali sono atrofizzati, l'addome è terminato da due o tre setole qualche volta lunghissime.

Molti sono i fatti degni di nota che la struttura ed i costumi delle Efimere, gli archetipi degli insetti offrono all'esame del naturalista. Parecchi sono stati di già accennati nei capitoli precedenti. Ricorderò tuttavia che le Efimere sono fra gli insetti quelli che hanno il maggior numero di mute (nel genere Chöleon sono 20) ed inoltre subiscono ancora una muta dopochè è già avvenuta la metamorfosi. La vita larvale delle Efimere è, per quanto se ne sa, di tre anni, quella dell'insetto perfetto è invece di poche ore.

Qualche volta le Efimere si sviluppano in cosi grande quantità da poter servire come eccellente concime.

La specie più generalmente nota è la $E$. vulgata Linn.

Le Efimere sono sparse per tutta la terra, ma, salvo le specie Europee, sono ancora poco conosciute.

LIBELLULIDI.

Distinguono gli insetti di questa famiglia le 4 ali di eguale lunghezza fra loro e fittamente reticolate, e il relativamente grande sviluppo dell'addome, il quale in qualche caso pareggia in lunghezza l'apertura delle ali.

Il capo è relativamente grande ed assai mobile, gli occhi sono grandissimi e sferici, qualche volta riuniti fra loro verso la parte superiore del capo. Gli ocelli sono in numero vario.

Le antenne sono corte e hanno al più sei o sette articoli. L'apparato boccale è in generale forte, robusto e foggiato per mordere. Le zampe non sono molto sviluppate; i maschi di qualche genere (Platycnemis) presentano delle espansioni membranose nelle zampe mediane e nelle posteriori.

L'addome, come ho già detto, è molto lungo, conta dieci segmenti, ed ora è appiattito, ora, come si osserva nel massimo numero dei casi, è cilindroide. L'ultimo segmento porta delle appendici anali speciali, sviluppate soprattutto nei maschi e che servono a questi per tener ferma la femmina nell'accoppiamento. 
I Libellulidi sono carnivori tanto nello stato larvale quanto nello stato perfetto. Essi sono pure legati strettamente all'acqua; le loro larve, che hanno in generale l'aspetto di grossi ragni pelosi di color bruno o grigio, vivono nelle acque degli stagni e delle pozzanghere ed amano i fondi fangosi.

In primavera sono fra i primi insetti che animano gli stagni.

Le larve delle libellule sono notevoli soprattutto per la loro doppia maniera di respirazione. Possono respirare cioè o l'aria libera dell'atmosfera o l'aria sciolta nell'acqua. Presentano perciò ad un tempo le stimme (due paia nel protorace e nel metatorace) e le branchie, le quali possono essere o esterne o interne ed intestinali come ho già detto. La respirazione branchiale pare sia la più attiva e la più importante.

Rispetto alle viscere noterò la mancanza delle ghiandole salivari e la poca centralizzazione del sistema nervoso, carattere di inferiorità rispetto agli altri insetti.

I colori dei Libellulidi sono eleganti e variati, qualche specie ha bellissimi riflessi metallici. In qualche caso (Calopteryx) i sessi sono fra loro diversi nel colore.

I Libellulidi finalmente sono sparsi per tutta la terra, e si spingono fino nelle regioni più settentrionali dell'Europa, in Lapponia. Sui monti tuttavia non salgono molto in alto e non credo oltrepassino i 2500 metri. Molte specie sono potentissime volatrici e non raramente compiono delle migrazioni.

I Libellulidi possono venire divisi in tre sotto famiglie: $\mathrm{Ca}$ lopterigini, Eschnini e Libellulini.

Le ali anteriori e posteriori egualmente sviluppate e diverse di colore nel maschio e nella femmina caratterizzano la prima sotto famiglia.

Ricorderò il genere Calopteryx di cui le specie dai colori vivaci ed eleganti e dal volo leggero e grazioso sono sparse in Europa (non sono rare fra noi lungo le acque correnti il C. virgo e il C. splendens), in Africa, in Asia e nell'America settentrionale, ed il genere Agrion che comprende un grande numero di specie, fra le quali si hanno le forme più piccole di tutti i Libellulidi. In Europa è comunissimo l' $A$. puella Linn.

Il genere Mecitogaster Rambur dell'America meridionale, affine per molti rispetti al genere Agrion, merita di essere 
menzionato per la sua grande mole. Il $M$. linearis Fabr. del Brasile ha l'addome lungo 160 millimetri.

Gli Eschnini hanno le ali posteriori più larghe alla base che non le anteriori.

Il genere Gomphus Leach. presenta il capo depresso, gli occhi separati e le antenne di quattro articoli. Le sue specie, di mole mediocre, sono sparse per tutta l'Europa ed anche nell'Asia settentrionale, nell'Asia minore e nell'Africa settentrionale; è specie comune il G. vulgatissimus Linn.

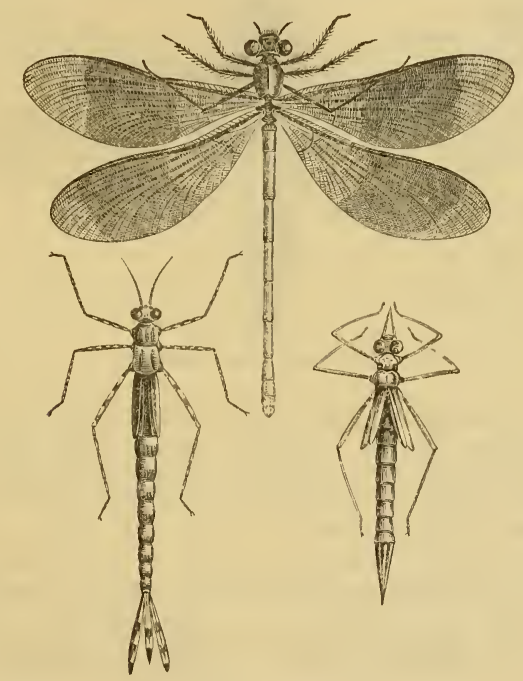

Fig. 52. Caloplerix splendens in vari stadi di sviluppo.

Il genere Aeschna Fabr. si distingue dal precedente soprattutto per avere gli occhi riuniti fra loro sulla parte superiore del capo.

Le specie di questo genere hanno mole relativamente grande, e sono ornate di eleganti colori. Nei maschi predominano i toni azzurri, nelle femmine invece $\mathrm{i}$ toni gialli e verdi. Hanno un volo rapido e lungo e si trovano principalmente attorno agli stagni o lungo le strade fiancheggiate da rigagnoli e da siepi. E specie comune fra noi l'Ae. maculatissima. 
Il capo globoso, gli occhi molto grandi, l'addome generalmente grosso, ora cilindrico, ora alquanto appiattito con cinque rialzamenti longitudinali, distinguono i Libellulini dai precedenti Libellulidi.

Il genere Libellula comprende un grande numero di specie sparse per tutta la terra. Il torace è in generale ricoperto da peli, le ali sono eguali fra loro. I colori sono meno eleganti di quelli del genere Aeschna. In molte specie, soprattutto nei

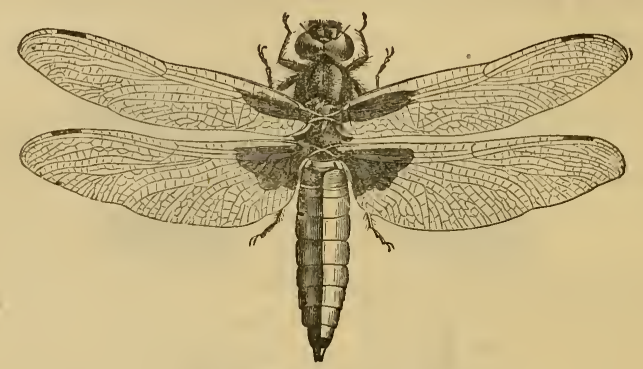

Fig. 53. Libellula depressa.

maschi, si osserva un'abbondante secrezióne di cera in forma di una polvere azzurra che copre buona parte dell'addome. Sono specie frequenti e note la $L$. quadrimaculata Linn., la L. depressa Linn., ecc.

Il genere Cordulia Leach., di cui è tipo la C. metallica V. Lind. dell'Europa settentrionale e centrale, è caratterizzato dall'avere gli occhi molti grossi e nella metà circa del margine posteriore un prolungamento e una piccola scavazione. 


\section{Bibliografia.}

Gli scritti intorno agli Ortotteri sono moltissimi e troppo lungo sarebbe il citarli tutti. Io mi limito ad indicare i più importanti: ricorrendo alle opere qui citate si possono avere indicazioni bibliografiche più ampie.

A. Serville. - Histoire naturelle des Insectes Ortoptères. Paris, 1839.

T. Charpentier. - Orthoptera descripta et depicta. Leipzig, 1841.

L. H. Fischer. - Orthoptera europea. Leipzig, 1853.

L. Dufour. - Recherches anat. et physiol. sur les Orthoptères. Parigi, 1841.

Selys Longchamps. - Catalogue raisonné des Orthoptères de Belgique. Bruxelles, 1862.

H. Nicoler. - Recherches pour servir à l'histoire naturelle des Podurelles. Nouv. Mém. de la Soc. Helvét. de Sc. Nat., vol. VI, 1842.

Mernerr. - Campodeae Natur. Tidsshrift., $3^{\mathrm{a}}$ serie, vol. III, 1865.

G. Gené. - Saggio di una monografia delle forficole indigene. Ann. Sc. Nat. Regn. Lomb. Venet., 1832, vol. II.

I. O. Westwood. - Catalogue of Orthopterous Insects in the collection of the Brit. Mus. London, 1859.

G. B. Grax. - Synopsis of the species of Insects belonging to the family of Phasmidae. London, 1835.

P. Huber. - Mémoire pour servir à l'histoire des Psoques. Mém. de la Soc. de phy. et d'hist. nat. de Genève, vol. X, 1843.

F. Muller. - Beiträge zur kentniss der Termiten. Jen. nat. Zeitschrift., volume VII, 1873.

Selys Longschamps et Hagen. - Revue des Odonates d'Europe. Bruxelles, 1854 1857.

A. Gestaecker. - Zur Morphologie der Orthoptera amphibiotica. Berlin, 1873.

J. De Bellesme. - Des Phénomènes Physiologiques de la métamorphose chez la Libellule déprimée. Paris, 1878.

Brunner von Watrenwru. - Die morphologische Bedeutung der Segmente speciell des Hinterleibes, bei den Orthopteren. Verh. der. Zool. bot. Gesell., Wien 1876.

- Monographie der Phaneropteriden, Wien 1878. 


\section{XVI.}

\section{NEVROTTERI}

Insetti con apparato boccale foggiato per masticare. Metamorfosi compiute.

L'ordine dei Nevrotteri, creato da Linneo, considerando principalmente la struttura delle ali (veupón nervatura e $\pi \tau \in \rho o ́ v$ ala), è molto difficile da essere caratterizzato e delimitato. Questo ordine da Linneo in poi subi molte vicende, ed anche al giorno d'oggi i naturalisti non sono d'accordo intorno alle forme che lo debbono costituire. Causa di queste discrepanze sono le affinità molto spiccate che i Nevrotteri presentano ad un tempo coi Lepidotteri e cogli Ortotteri.

I Nevrotteri, come vengono intesi dal-Claus, si distinguono tuttavia dagli Ortotteri facilmente per un carattere importantissimo, per avere cioè metamorfosi compiute, mentre negli Ortotteri le metamorfosi sono incompiute. Il complesso dell'interna struttura distingue pure i Nevrotteri dai Lepidotteri, ai quali si avvicinano per avere spesso le ali ricoperte in parte da piccole squame, e per avere in qualche caso (Friganee) l'apparato boccale masticatore che tende a trasformarsi in apparato succiatore.

Il capo nei Nevrotteri è sempre distinto dal resto del corpo. E molto grande nei Sialidi e soprattutto nel genere Coryclalis e nei Mirmeleoni, è invece piccolo nei Friganidi. Le antenne sono sempre ben sviluppate, ora allungate, con molti articoli

- e filiformi, come nella massima parte dei casi, ora rigonfie nell'estremità superiore come nei Mirmeleoni.

Lo sviluppo delle mandibole e delle altre parti della bocca varia assai nelle diverse famiglie.

L'apparato masticatore è robusto e le mandibole sono molto sviluppate nei Sialidi, ed in generale in tutti i Planipenni. 
Nel genere Corydalis le mandibole del maschio si sviluppano straordinariamente e ricordano ciò che si osserva nei maschi di molti Coleotteri.

Nei Tricotteri, come ho già detto, l'apparato masticatore tende a trasformarsi in un apparato succiatore. Le mandibole, vale a dire, si atrofizzano e le mascelle saldandosi col labbro inferiore costituiscono una tromba aspirante.

Gli occhi esistono sempre e sono più o meno sviluppati secondo le famiglie: gli ocelli ora vi sono, ora mancano.

Il protorace è mobile e più o meno sviluppato.

L'addome conta otto o nove segmenti per lo più molto allungati, in guisa che questa parte del corpo dei Nevrotteri ricorda le Libellule fra i Pseudonevrotteri. Qualche volta $(\mathrm{Pa}-$ norpidi) gli ultimi tre segmenti dell'addome sono molto stretti e terminano nel maschio con una tanaglia destinata a tener ferma la femmina nell'accoppiamento.

Le zampe sono relativamente meno sviluppate che non nella massima parte degli Ortotteri. I tarsi hanno cinque articoli. In qualche genere (Mantispa) le zampe anteriori sono sviluppatissime, foggiate come quelle delle Mantidi e trasformate in organi di presa.

Nel genere Boreus (Panorpidi) le zampe posteriori sono allungate ed atte al salto.

Nei Tricotteri le tibie presentano spesso un numero vario di sproni o di spine, le quali hanno molta importanza nella classificazione di questi insetti.

Le ali, meno in qualche caso in cui sono atrofizzate (nel genere Boreus Latr. e nel Bittacus apterus. M. Lachl. della California), sono sempre ben sviluppate, ed anzi proporzionatamente si puó dire che il loro sviluppo supera quello delle ali della maggior parte degli Ortotteri.

Le ali sono sempre membranose e presentano più o meno numerose nervature. Ora le due paia di ali sono fra loro quasi egualmente sviluppate (genere Hemerobius, Mirmeleo, ecc.), ora invece, come si osserva nella maggior parte dei casi, il paio anteriore è un po' più lungo ed un po' più grande del posteriore. Nel genere Nemoptera le ali posteriori sono molto allungate ed hanno l'aspetto di due code variamente foggiate nella loro estremità inferiore. 
Nei Tricotteri le ali sono ricoperte in parte da squame e da peli simili a quelli delle ali dei Lepidotteri.

Le ali posteriori possono, quando l'insetto è in riposo, essere ripiegate (Tricotteri in generale) o non (Planipenni).

Per ciò che è dell'interna struttura noterò che il sistema nervoso è foggiato sul piano di quello degli Ortotteri e che in generale il ventriglio è ben sviluppato. I Nevrotteri sono carnivori.

Le metamorfosi sono compiute e in molti casi lo stadio di ninfa si passa in un bozzolo come nei Lepidotteri: le ninfe tuttavia, contrariamente a quanto avviene nei Lepidotteri, alla fine del periodo di ninfa possono muoversi e cercare un luogo propizio per compiere il passaggio allo stato perfetto.

Lo sviluppo di una buona parte dei Nevrotteri (Tricotteri) è essenzialmente legato all'acqua. Molto vari e curiosi sono gli astucci che le larve acquatiche costruiscono, ora servendosi di pietruzze che impastano assieme, ora di pezzettini di piante acquatiche, ora di conchigliette, ecc. Talvolta la forma di questi astucci imita quella delle conchiglie di certi molluschi. Gli astucci larvali di qualche genere americano imitano le conchiglie dei Dentalium, quelli del genere Helicopsiche le comuni chiocciolette dei nostri fossi.

Le larve delle Friganee hanno un numero vario di trachee branchiali filiformi collocate ai lati dell'addome, che servono per respirare l'aria sciolta nell'acqua.

Le larve dei Planipenni vivono o nella terra umida ( $\mathrm{Pa}$ norpidi) o sotto la scorza degli alberi (genere Raphidia) o nei. luoghi caldi e sabbiosi (Mirmeleo).

Non molto spiccati sono nei Nevrotteri i caratteri sessuali secondari. Non rari sembrano i casi di mimismo con insetti di altri ordini, il Bittacus tipularius, ad esempio, ha tutto l'aspetto dei Ditteri del genere Tipula: le zampe del genere Bittacus sono invero sottili e molto lunghe, il corpo è lineare ed allungato, le ali sono strette, allungate e peduncolate, ecc. (1). Cosi pure il genere Boreus rassomiglia a certi

(1) Le specie del genere Bittacus si nutrono di piccoli insetti, che essi aspettano al varco, stando in agguato sospesi ad un ramoscello o ad una foglia di una pianta. La loro rassomiglianza colle Tipule inoffensive torna loro percio molto giovevole. 
Ortotteri privi di ali della famiglia dei Locustini. L'addome infatti nel genere Boreus è molto grosso: la femmina ha un ovopositore fatto a sciabola: le zampe posteriori sono atte al salto, ecc.

I colori dei Nevrotteri non sono molto appariscenti ed eleganti. Predominano in essi il bruno, il giallognolo ed il bianchiccio. I Nevrotteri sono sparsi per tutta la terra, ma fino ad ora non sono state studiate diligentemente che le specie europee e quelle dell'America settentrionale.

Taoola di classificazione dei Neorotteri.

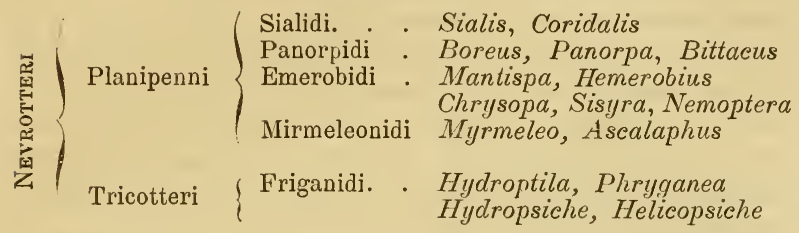

A. Ali anteriori e posteriori simili fra loro e che non si ripiegano. Apparato boccale foggiato per masticare . Planipenni. a. Antenne allungate e filiformi.

$b$. Mascelle inferiori non saldate col mento.

c. Mascelle inferiori con una galea ed un lobo masticatore.

Sialidi.

$e^{\prime}$. Mascelle inferiori con un lobo esterno biarticolato.

Emerobidi.

$b^{\prime}$. Mascelle inferiori saldate col mento e formanti una specie di becco . . . . . . Panorpidi.

$a^{\prime}$. Antenne che si ingrossano verso la loro estremità superiore, claviformi . . . . . Mirmeleonidi. $A^{\prime}$. Ali ricoperte da squame e da peli: le ali inferiori nello stato di riposo stanno ripiegate (Tricotteri) . . Friganidi.

\section{Planipenat.}

Nel genere Sialis le antenne sono lunghe: gli ocelli mancano: il protorace è quadrangolare: le ali posteriori sono più larghe alla loro base che non le anteriori. Le zampe sono relativamente allungate. Le larve delle specie di questo genere sono acquatiche e presentano lateralmente dei filamenti branchiali esterni. Avviene non raramente di trovare larve di Sialis 
negli astucci delle Friganee. La specie più nota di questo genere si è la S. lutaria Linn.

Merita di essere ricordato il genere Chaudiodes, che comprende una ventina di specie che abitano l'America, l'Asia orientale, l'Africa australe, la Nuova Zelanda e l'Australia, e che è caratterizzato dalle antenne pettinate.

Il genere Corydalis esclusivamente americano e che comprende specie relativamente di grande mole, è caratterizzato, come ho già detto, dal grande sviluppo delle mandibole nel maschio. Vi hanno in questo genere tre ocelli. Le antenne sono dentate nei maschi (articoli delle antenne 60 circa), filiformi nelle femmine (articoli delle antenne 54 circa). Le ali sono molto sviluppate; l'addome è allungato e nel maschio l'ultimo segmento è terminato da appendici fatte a mo' di tanaglie. Predomina sul corpo il colore giallo-bruno. Le larve sono carnivore; fanno vita acquatica ed hanno dei filamenti branchiali esterni (1). La specie più anticamente nota di questo genere è la C. cornuta Linn.

Raphidia Linn. Capo relativamente grande, cordiforme ed inclinato: tre ocelli sul vertice: antenne filiformi, e che ripiegate lungo il corpo non giungono alla fine del protorace: torace lungo quasi come la metà del corpo: protorace molto sviluppato ed alquanto più stretto del capo. Le larve di questo genere vivono per lo più sotto alla corteccia delle quercie o dei pioppi. Specie note e sparse in quasi tutta l'Europa sono la $R$. notata Fabr., la $R$. ophiapsis e la $R$. xanthostigma, ecc.

Panorpa. Carattere principale di questo genere, oltre a quelli già citati dell'apparato boccale, si è quello dell'addome conico, gli ultimi anelli foggiati nei maschi a mo' di una coda di cui l'ultimo articolo è ingrossato e termina con due appendici a mo' di tanaglie: nella femmina l'addome si allunga e si assottiglia in modo da ricordare l'addome delle Tipule fra i Ditteri. Le ali sono grandi, eguali fra loro e per lo più macchiettate di bruno. Numerose sono le specie conosciute del ge-

(1) Un fatto degno di nota si osserva nella larva delle Corydalis. Le larve di questo genere presentano delle differenze sessuali secondarie abbastanza spiccate: il capo, cioè, e le mandibole sono nelle larve dei maschi più larghe e più robuste che non nelle larve delle femmine. 
nere Panorpa e sono sparse nelle varie regioni della terra. Fra noi è comunissima la $P$. communis Linn. Le Panorpe si nutrono, a quanto pare, d'insetti morti (1). Le larve e le ninfe delle Panorpe vivono nella terra umida a una profonditá relativamente grande.

Il genere Boreus Latr. è notevole per le ali atrofizzate, cosa non frequente nei Nevrotteri, per la presenza di un ovopositore nella femmina e per le zampe posteriori atte al salto. Le specie di questo genere abitano principalmente $\mathrm{i}$ paesi freddi e le elevate regioni dei monti. La specie più nota è il B. hiemalis Linn. non raro in Svizzera, in Germania, sulle Alpi, ecc. Mentre le specie del genere Boreus sono proprie delle regioni fredde, quelle invece del genere Bittacus Latr. appartengono alle regioni equatoriali. Notevole è questo genere, come ho già detto, per la rassomiglianza delle sue specie colle Tipule. In Europa trovasi il B. italicus Muell.

Menzionerò ancora il genere Chorista Klug dell'Australia, in cui la bocca è priva di rostro, contrariamente a ciò che si osserva in tutte le altre forme appartenenti ai panorpidi.

Mantispa Illig. Le antenne sono corte e moniliformi : mancano gli ocelli ; il protorace è allungato e cilindrico; le ali sono quasi al tutto simili fra loro; le zampe anteriori sono molto sviluppate e sono come nelle Mantidi foggiate ad organi di presa. In Europa vi hanno due specie di questo genere la $M$. pagana Fabr., e la $M$. perla Pallas. Lo sviluppo della prima specie citata venne fatto conoscere ultimamente dal Brauer (2) ed è curiosissimo, presentandoci esso fatti di parassitismo e di ipermetamorfosi analogi a quelli che si osservano nei generi Melöe e Sitaris fra i Coleotteri.

Chrysopa Leach. Le antenne sono in generale lunghe (piu nei maschi che non nelle femmine) e constano di un numero di articoli che varia da settanta a cento, ed hanno due o più peluzzi. Gli occhi sono grandi, globosi e ornati di splendidi colori, verdi, dorati o bruno-metallici. Le uova della specie del genere Chrysopa sono, cosa notevole, peduncolate. Le

(1) Io stesso osservai ripetutamente, nel contorno di Torino, vari individui di panorpe intenti a divorare $\mathrm{i}$ cadaveri delle comuni melolonte.

(2) Verhandl. zool. Gotan. Gesell. in Wien., 1869. 
Crisope si nutrono di insetti e più particolarmente di Aphis. La specie di Crisopa più comune in Europa, è la $C$. vulgaris Schn. (C. perla Autc.).

Hemerobius Linn. Antenne moniliformi : ocelli mancanti: zampe cilindriche o fusiformi: ali ora trasparenti e ialine, ora, come si osserva nel maggior numero dei casi, variamente colorate e macchiettate e alquanto pelose. Si conoscono una sessantina di specie appartenenti a questo genere, la maggior parte si trovano sulle conifere in Europa e nell'America settentrionale. Si trovano pure specie di questo genere in Australia, nell'India, nel Brasile, nel Chili, ecc. Le larve vivono pure sulle conifere e si nutrono di afidi.

Relativamente numerose sono le specie fossili di Hemerobius nell'ambra e nei terreni giurassici e terziari.

Il genere Sisyra Burm. merita di essere menzionato soprattutto per la vita acquatica delle sue larve, cosa eccezionale negli Emerobidi, e per le ali che sono quasi al tutto prive di nervature. Le antenne in questo genere sono moniliformi: gli ocelli mancano; il protorace è corto: le zampe sono cilindriche : il corpo è ricoperto da peli.

Le specie conosciute di questo genere, sono fino ad ora in piccolo numero, e sono proprie dell'Europa e dell'America settentrionale. Esse vivono lungo i fossi in prossimitì dell'acqua. La larva della S. fuscata Fabr. venne descritta per la prima volta dal Grube e dal Westwood (1). Quest'ultimo l'aveva denominata Branchiotoma spongillae, non sapendo di aver sotto agli occhi una larva di Sysira.

Osmylus Latr. Questo genere è molto importante, perchè segna un passaggio per la sua struttura e per le sue larve a vita semi-acquatica da una parte ai generi Chrysopa ed Hemerobius, e dall'altra al genere Sisyra. Il genere Osmylus si distingue dai due precedenti soprattutto per la presenza di tre ocelli. Le ali hanno numerose nervature ricoperte da lunghi e folti peli. Vennero sino ad ora trovate specie di questo ge-

(1) F. Grube a Beschreibung einer auffallenden, an Süsswasserschwämmen lebenden Larve». Wieg. Archiv. f. Natur., vol. XVII, 1843.

J. 0. Westwood « Description of some Insects which inhabit the tissue of Spongilla fluviatilis. Trans. of Ent. Soc. of London, vol. III, 1841-43. 
nere in Europa, nelle Indie, in Tasmania e nella Nuova Zelanda. I costumi e lo sviluppo di questo genere vennero studiati principalmente dal Brauer (1) e dall'Hagen (2).

Il genere Coniopteryx Halid. comprende forme molto piccole ancora poco note, che vennero fino ad ora trovate nell'Europa settentrionale e centrale e nell'America del Nord. Le ali sono ricoperte da uno straterello pulverulento di cera. La specie tipica è il C. tineiformis Curt. che si trova sulle conifere. La larva ha molta rassomiglianza con quelle delle specie del genere Hemerobius.

Il genere Nemoptera Latr. viene facilmente distinto da tutti gli altri menzionati per le ali posteriori molto allungate, lineari

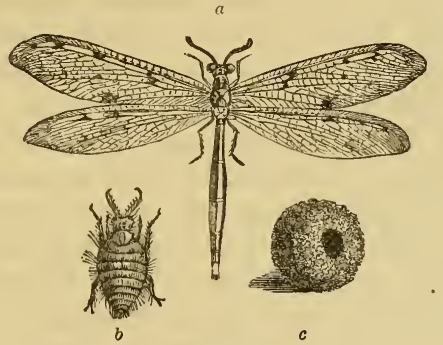

Fig. 54. Myrmeleo formicarius.

$a$ insetto perfetto $-b$ larva $-c$ oozzolo contenente la ninfa.

e rigonfiate alquanto alla loro estremità inferiore e per l'apparato boccale foggiato a mo' di un becco (diverso tuttavia da quello delle Panorpe). Le specie di questo genere sono state trovate nelle regioni calde dell'antico continente, Spagna, Portogallo, Africa Settentrionale, Asia Minore, Arabia, ecc. Poco si sa ancora intorno ai loro costumi ed intorno alla loro struttura.

Interessantissimo pei suoi costumi è il genere Myrmeleo, tipo della famiglia dei Mirmeleoni, caratterizzata nel modo che è già stato detto. Il corpo è quasi al tutto privo di peli : le ali sono per lo più macchiettate. Numerosissime e molto diffi-

(1) Wiegmann's Archiv., 1851.

(2) Curtis, British Entomology, vol. XI, 1834. 
cili da essere studiate sono le specie di questo genere, che si trova in tutte le regioni temperate e soprattutto calde della terra (1). Le larve vivono nella sabbia, e si scavano una sorta l'imbuto, in fondo al quale aspettano gl' insetti imprudenti, di cui succhiano gli umori, che si lasciano scivolare lungo la china dell'imbuto stesso (2).

Le larve intessono un bozzolo sferoide, nel quale passano lo stadio di ninfa. L'insetto perfetto ricorda l'aspetto delle libellule; si distingue tuttavia da questo, oltre che per le varie particolarità di struttura, anche pel suo volo lento e pel gralevole odore di rosa ch'esso tramanda.

Il genere Ascalaphus si distingue dal genere precedente per la lunghezza delle antenne terminate nella estremità superiore a clava. Gli occhi ora sono divisi in due da un solco (Schizophthalmi di Lefevre), ora invece sono intieri come al solito (Holophthalmi di Lefevre). Per lo più il torace porta molti peli. Le ali sono relativamente grandi e spesso presentano colori vivaci ed eleganti, le zampe sono forti, robuste e coperte di peli rigidi; le mandibole sono ben sviluppate e forti e in rapporto colla natura insettivora degli ascalafi. Le larre rassomigliano pel complesso dei caratteri a quelle dei Formicaleoni, ma non iscavano imbuti ed abitano fra i muschi nei prati stando in agguato ad aspettare la preda. Non sono rari l'A. italicus Latr., l'A. lacteus Brullé, ecc.

Menzionerò ancora il genere Haplogenius Burm. dell'America centrale, appartenente alla categoria degli Ascalafi oloftalmi, il quale presenta le ali, nello stato di riposo, come le libellule, vale a dire disposte orizzontalmente e non lungo il corpo come negli altri Ascalafi.

TRICOTTERI.

I Tricotteri sono distinti, oltre che dai caratteri già menzionati, anche dall'avere le larve, che fanno vita acquatica e si costruiscono degl'involucri, dei gusci tubiformi o diritti, o avvolti a spirale, servendosi o di piccole pietruzze, o di

(1) Si veda una descrizione bellissima dei costumi di queste larve nell'opera ripetutamente citata del Réaumur.

(2) Nel contorno di Torino sono località buone per osservare i costumi dei formicaleoni, le rive sabbiose della Stura nelle giornate calde e di bel sole. 
frammenti di piante acquatiche o di piccole conchigliette. Lo addome delle larve porta un numero vario di filamenti branchiali esterni. L'apparato boccale è bene sviluppato e masticatore nelle larve; spesso rudimentale e foggiato per succiare negl'insetti perfetti. I Tricotteri, come ho già accennato, sono affini per molti rispetti ai Lepidotteri. Sono sparsi per tutta la terra, ma salvo le specie europee e dell'America del Nord, sono poco conosciuti.

Il genere Sericostoma Latr. è degno di nota pel grande sviluppo dei palpi mascellari dei maschi.

Al gruppo dei Sericostomidi appartiene un genere, il genere Helycopsyche notevole per la forma degli astucchi larvali, che hanno in tutto la forma di una piccola conchiglietta dei generi Helix, Physa, Valvata, ecc. Gli astucchi di queste friganee vennero infatti descritti come conchiglie di molluschi e creduti tali per un certo tempo. Le specie del genere $\mathrm{He}$ lycopsyche si trovano sparse forse in quasi tutte le regioni calde. In Europa si trovano in Svizzera, in Portogallo, in Italia (1), in Corsica, ecc. lungo i ruscelli. Gl'insetti perfetti della specie di questo genere erano intieramente sconosciuti fino a questi ultimi tempi, non essendo mai nessuno riuscito ad ottenere lo sviluppo delle larve. In sulla fine dell'anno scorso (1878) tuttavia il signor G. Tassinari per primo ottenue tre individui allo stato perfetto, che trasmise al professor Targioni-Tozzetti, il quale, studiatili, li riconobbe appartenere ad una specie e forse anche ad un genere nuovo (2).

Limnophilus Burm. Questo genere comprende molte specie che si trovano nell'Asia e nell'Europa, nell'America setten-

(1) In Italia vennero trovate in varie località. In Piemonte vennero trovate, credo per la prima volta, in un ruscelletto presso Bollengo, in su quel d'Ivrea, nell'estate del 1875 .

(2) Riferisco la descrizione colle parole stesse dell'Autore stampata, colla figura dell'insetto che il T. Tozzetti chiama Helycopsyche agglutinans, nei rendiconti della Società Entomologica Italiana (Seduta del 24 novembre 1878): " $1^{\circ}$ Palpus maxillaris, 4 articulatus, art. basilari elliptico crassiore, $2^{\circ}$ apice inferne inflato asimmetrico, $3^{\circ}$ omnium longiore; flagello incomplete, irregulariter 12-13 articulato, art. basali $2^{\circ}+3^{\circ}+4^{\circ}$ subaequali. Palpus labialis, maxillaris minor, 3 articolatus, flagello, basi subaequali, maxillari persimili. Pedes antici breviores; tibia apice bibracteata. Pedes 2 ' 3 ' anticor. longiores, 
trionale e anche, fatto che si osserva anche in altri gruppi d'insetti (ad esempio, nei carabici fra i Coleotteri), nel Chili e nelle isole Falkland.

La specie più comune in Europa è il L. rhombicus Linn., il quale ha il capo ed il torace rossastri e l'addome rossastro superiormente, verdognolo chiaro inferiormente. Le ali sono relativamente grandi, e di color giallo-pagliarino chiaro, con una larga macchia biancastra, larga, orlata di rosso e che spesso ha la forma di un rombo.

Phryganea Linn. Questo genere che, oltre ai caratteri giå accennati del gruppo, presenta le antenne filiformi e più lunghe

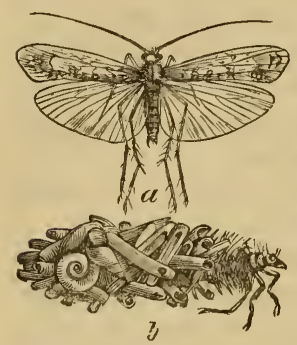
delle ali che sono ricoperte di peli; comprende molte specie sparse in quasi tutte le regioni della Terra. In Europa sono comuni la $P$. striata, la $P$. grandis, ecc. (1). Affine al genere Phryganea è il genere Neusonia Leach. (Holostomis Murh.), che comprende forme di mole relativamente grande, dalle ali larghe e quasi al tutto prive di peli, proprie delle regioni settenFig. 55. Fryganea flavicornis.
$a$ insetto perfetto - b larva entro l'astuccio.

$N$. phalaenoides Linn.

Hydropsyche. Questo ed altri generi (Macronema Pictet. ecc.) di Tricotteri appartenenti allo stesso gruppo, sono distinti per avere le antenne sottili e per lo più molto lunghe. Le larve vivono in astucchi fissati al fondo dell'acqua. L'insetto adulto ricorda moltissimo certi Lepidotteri. L'H. atomaria

tibia medio et apice bibracteata; ordo longitudinis 2.3.4 (nece 2.2.4). Tarsus biunguiculatus, arolio parvulo inferno. Alae anticae abdominis dimidio longiores, posticarum latiores, nervibus nervulisque liberis, areolisque apertis, retinaculis in margine costali alarum posticarum nullis. Foem. abdom. 7, anulatum, ovipositore styliformi complexo, dimidio breviori, terminatum. Mas adhuc caret».

(1) Nel contorno di Torino i ruscelli che discendono dalla collina sono ricchissimi di Friganee, le quali amano, in generale, le acque limpide alquanto fredde e scorrenti. Le larve di Friganee sono pure frequenti nei ristagni dei torrenti alpini. 
Pictet o l'H. nebulosa Pictet non sono rare, a quanto pare, in Europa.

Il Claus (1), di cui seguiamo la classificazione, colloca alla fine dei Nevrotteri il gruppo curiosissimo degli Strepsitteri. Egli li definisce cosi : "Insetti con ali anteriori poco sviluppate e avvolte alla loro estremità : con ali posteriori pieghettate longitudinalmente: con apparato boccale rudimentale. Le femmine sono attere ed apode. Vivono parassiti allo stato di larva sul corpo di vari Imenotteri ».

Ricorderò il genere Xenos in cui gli occhi sono leggermente peduncolati, ed il genere Stylops in cui i peduncoli sono più distinti e sviluppati.

Bibliografia (2).

RAMbur. - Histoire nat. des Insect. nevroptères. Parigi, 1842.

F. Brauer et F. Löw. - Neuroptera austriaca. Vienna, 1857.

Picter. - Histoire nat. des Nevroptères. Ginevra, 1841-43.

- Recherches pour servir à l'histoire et à l'anatomie des Phryganides. Ginevra, 1834.

- Synopsis des Nevroptères d'Espagne. Ginevra, 1865.

G. F. Schneider. - Monographia generis Raphidis Linn. Breslau, 1843.

- Symbolae ad monographiam generis Chrysopae Leach.

I. O. Westwood. - On the genus Mantispa. Trans. ent. soc., $2^{\mathrm{a}}$ serie, vol. I.

- Monograph of the genus Panorpa. Trans. ent. soc., vol. IV.

F. KLUG. - Versuch einer systematischen Feststellung der Familiae Panorpatae. Berlino, 1836.

H. HAGEN. - Die larven von Myrmeleon. Stett. entomol. Zeit., 1873. vol. XXXIV.

(1) Opera citata.

(2) Vedi nota in principio della bibliografia degli Ortotteri. 
Mac Lachlan. - Notes sur les Phryganides. Ann. Soc. Entomol. de Belg., volume XIII, 1869-70.

- A monographic Revision and Synopsis of the Trichoptera of the European Fauna. Ann. Soc. Ent. de Belg.

- Synopsis of the Species of Panorpa occuring in Europa, ecc. Trans. Soc. Ent. of London, 1869.

Meyer-Dür. - Die Neuropteren Fauna der Schweiz, 1875.

H HGEN. - Synopsis of the British Phryganidae. Entomol. Annal. for., 1859-61.

Kolenati. - Genera et species Trichopterorum. Pars. I. Abh. der Böhmischen Gesell. der Wiss., 6a serie, 1851. - Pars. II. Nouv. Mrém. de la Soc. de Nat. de Moscou, 1859.

- Hemerobidarum Synopsis synonymica. Ent. Zeit. Stettin., 1866.

I. O. Westwood. - Description of a strepsipterous insect. Trans. Entom. Soc. London, vol. I.

Cortis. - British entomology. Londra, 1849.

V. Síвоцd. - Ueber Strepsiptera. Archiv. für Naturg., vol. IX, 1843.

- Ueber Paedogenesis der Strepsipteren. Zeitsch. für wissen. Zool, vol. XX. 1870 . 


\section{XVII.}

\section{RINCOTI}

Apparato boceale foggiato per pungere e per succhiare e allun. gato a mo' di becco. Metamorfosi incompinte.

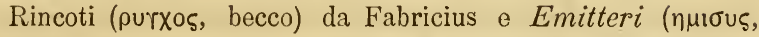
metà e $\pi \tau \in \rho o v$ ala) da Linneo vennero chiamati gli animali costituenti per noi il terzo ordine degl'insetti. I Rincoti formano un gruppo di insetti non molto naturale e che, nelle varie forme che comprende, ci presenta una serie di modificazioni che ricordano gli Ortotteri. Riesce difficile per la grande varietà di forma dei Rincoti di dare dei caratteri generali precisi di questi insetti. Il capo varia molto nei Rincoti, ora è piccolo ed incassato nel torace (Tesseratoma, Acanthia, ecc.), ora di mediocre grandezza e unito al torace per mezzo di un peduncolo o collo più o meno lungo (genere Myschoris), ora è anteriormente intiero, ora invece è intaccato più o meno profondamente (genere Storthia), ora è dilatato lateralmente, ora invece nel mezzo dei lati presenta un ristringimento molto spiccato, ora è superiormente appiattito o leggermente convesso, ora porta due prolungamenti a mo' di corna ben evidenti (genere Ceratocoris); finalmente puó unirsi col torace e costituire le strutture stranissime dei Membracidi, o può allungarsi anteriormente come nei Cicadellidi.

Gli occhi sono in generale ben sviluppati: ora sono grossi come nella maggior parte dei Cicadidi, ora invece piccoli come nei Reduvidi, nei Coccidi, ecc. Si trovano raramente anche degli ocelli.

Le antenne, che variano pure assai nella forma, contano in generale uno scarso numero di articoli (fa eccezione il genere Monophlebus, in cui i maschi hanno le antenne di venti 
o venticinque articoli), questi tuttavia in qualche genere pigliano un grande sviluppo (generi Macrocheraia, Reduvius, Zelus, ecc.). Nella massima parte dei casi le antenne sono filiformi, qualche volta o l'ultimo articolo s'ingrossa a mo' di clava (genere Phyllomorphus), o il penultimo (genere Charicotes) e l'antipenultimo (genere Copius) presentano delle espansioni laterali molto spiccate, o $\mathrm{i}$ due primi sono molto ingrossati e gli ultimi esilissimi (genere Harpoceris), o finalmente tutti gli articoli sono ovoidi, ingrossati e ricoperti di peli (genere Phricodis).

Il torace è oltre ogni dire variabile di forma e troppo lungo, ed essendo poco profittevole, non potendo accompagnare la descrizione con figure, l'enumerare minutamente le varie sue strutture, mi limiterò ad accennare le principali. Esso è trasversale e più o meno incurvato in generale negli Atteri, nei Fitoftiri e nei Cicadellidi: è straordinariamente allungato posteriormente, tanto che non solo ricopre l'addome, ma lo oltrepassa spesso di un tratto notevole, nei Membracidi. In quest'insetti il torace si unisce pure anteriormente al capo e presenta delle espansioni dirette in alto, ora in forma di corna, ora di elmo, ora al tutto irregolari. Talvolta la struttura di queste parti è spugnosa. Negli Emitteri propriamente detti ora il torace è trasversale e leggermente convesso, ora presenta due corna o spine laterali (generi Trigonosoma, Asopus, Choricoteris, Piezomerus, Edessa), od anteriori (generi Menenotus, Pandarus, Dyrodis), o laterali e superiori ad un tempo (genere Zelus). Talvolta il torace ha l'aspetto spugnoso e presenta due espansioni membranose laterali pure spugnose (genere Tingis). Espansioni laterali molto strane e che dànno all'insetto l'aspetto di una foglia, si trovano ai lati del torace e delle elitre del genere Phyllomorphus. Il torace finalmente puó essere molto allungato e stretto, come ad esempio nei generi Neides, $R a$ natra, ecc. Lo scudetto in generale di forma triangolare puo essere al tutto nascosto o essere visibile esternamente ed avere uno sviluppo grandissimo, come si osserva in moltissime forme di Emitteri propriamente detti. Le ali, considerando l'ordine dei Rincoti complessivamente, non sono molto sviluppate, esse sono ora in numero di quattro, come si osserva nel massimo numero dei casi, ora di due come è il caso dei maschi dei 
generi Aspidiotus, Lecanium, ecc., fra i Coccidi, ora mancano intieramente (la massima parte delle femmine dei Coccidi) (1). Le ali anteriori sono nei Rincoti in generale un po' più dure e più vivacemente colorite delle posteriori. In qualche caso esse hanno intieramente l'aspetto delle elitre dei Coleotteri. In molti Rincoti rimane membranosa la parte inferiore del primo paio di ali. Le zampe sono in generale lunghe e sottili, e per la poca robustezza dell'articolazione coi segmenti toracici, facilmente si staccano. Gli articoli dei tarsi variano da due a tre. Ora le zampe sono sottili e molto lunghe, come nei generi Macrocheraia, Ploiaria, Neides, Ranatra, ecc., ora invece sono corte e relativamente robuste, come si osserva. in quasi tutti i Cicadellidi. Troviamo pure nei Rincoti le zampe anteriori trasformate in organi di presa (generi Nepa, Ranatra, ecc.), e le posteriori ora ingrossate ed atte al salto (molti Cicadari e generi $\mathrm{Pe}$ tascelidis, Omoscelis, ecc.), ora invece compresse lateralmente ed atte al nuoto (Notonecta, ecc.). Le zampe mediane e posteriori possono pure in qualche caso essere molto più

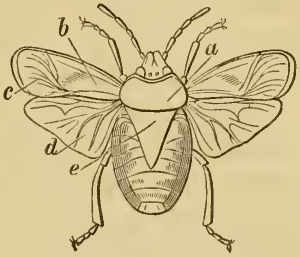

Fig. 56. Cimex oleraceus. $a$ protorace - $b$ porzione dura delle ali anteriori - $c$ porzione membranosa - $d$ ali posteriori meinbranose e nervature $-e$ scudetto. lunghe delle anteriori. Ciò si osserva principalmente nei generi Gerris, Halobates, ecc., le di cui specie corrono rapidissimamente sulla superficie dell'acqua. Le zampe posteriori finalmente presentano talvolta delle espansioni membranose speciali (generi Diactor e Anisoscelis).

Il numero dei segmenti dell'addome può essere di sei, di otto o di nove. La forma poi dell'addome è molto variabile. Esso è allungato e stretto nei generi Ranatra, Hydrometra, $M a$ crocheraia, Neides, ecc.; allargato ed appiattito in quasi tutti i Geocorisi; fortemente carenato in molti Cicadari. L'addome porta talvolta lateralmente delle espansioni molto sviluppate, che concorrono con quelle già accennate del torace a dare

(1) In qualche caso la mancanza delle ali è dovuta a metamorfosi regressive, come ad esempio nel genere Acanthia. 
all'insetto una determinata rassomiglianza protettrice. Le espansioni laterali dell'addome sono ben evidenti, ad esempio nel genere Phyllomorpha, ecc. Non infrequentemente, soprattutto fra i Cicadari, l'addome porta, come abbiamo già avuto occasione di osservare, dei lunghi filamenti di cera, che dànno all'insetto un aspetto strano.

Per ciò che è dell'anatomia interna possiamo osservare che le ghiandole salivari sono in generale molto sviluppate e lo stomaco è diviso spesso in tre parti. I vasi Malpighiani sono in numero di quattro. I Rincoti, come a tutti è noto, mandano in generale, quando vengono toccati, un odore disgustosissimo. Quest'odore è dovuto ad una sostanza preparata da ghiandole speciali collocate nella parte inferiore del mesotorace e del metatorace. Le metamorfosi dei Rincoti sono incompiute ed in ciò si avvicinano agli Ortotteri.

La riproduzione dei Rincoti ci presenta il fenomeno importantissimo della partenogenesi di cui abbiamo già parlato nel capitolo della riproduzione. I Rincoti si nutrono di sostanze liquide che tolgono succhiando dagli animali o dai vegetali. Nei Rincoti il fatto del parassitismo è molto spiccato (e ciò è un carattere d'inferiorità) e molte speciẹ riescono perciò nocevoli all'uomo, o perchè lo attaccano direttamente punzecchiandolo e succhiandogli il sangue, o perchè attaccano molte specie di vegetali che dall'uomo stesso rengono coltivate. $\mathrm{Ba}$ sterà che a questo proposito menzioni i generi Pediculus e Phylloxera. I Rincoti comprendono specie di grande mole. Le forme più grosse sono in generale acquaiuole. I colori, ch'essi presentano, sono per lo più vivaci ed eleganti e qualche specie delle regioni calde compete coi Coleotteri pei riflessi metallici delle sue ali. Nei Rincoti si osservano non solo casi spiccatissimi di rassomiglianze protettive nella forma (Phyllomorpha, aspetto di foglia) e nel colore (molte specie del genere Pentatoma), ma non sono eziandio rari i casi di mimismo con insetti di altri ordini. Cosi, ad esempio, il genere Neides ha l'aspetto del genere Tipula fra i Ditteri, il genere Eurybia ha il facies di un Buprestide fra i Coleotteri; il genere Araphis ricorda il genere Staphylinus pure fra i Coleotteri. Numerose specie di piccole Cicale infine rassomigliano moltissimo a piccoli Lepidotteri. 
Il numero delle specie conosciute è relativamente grande, tuttavia molte più rimangono da conoscersi, non essendo fino ad ora stata studiata un po' diligentemente che qualche regione dell'Europa. Non si può dir nulla quindi di generale e di sicuro intorno alla distribuzione geografica dei Rincoti.

\section{Tavola di elassificazione dei Rineoti.}

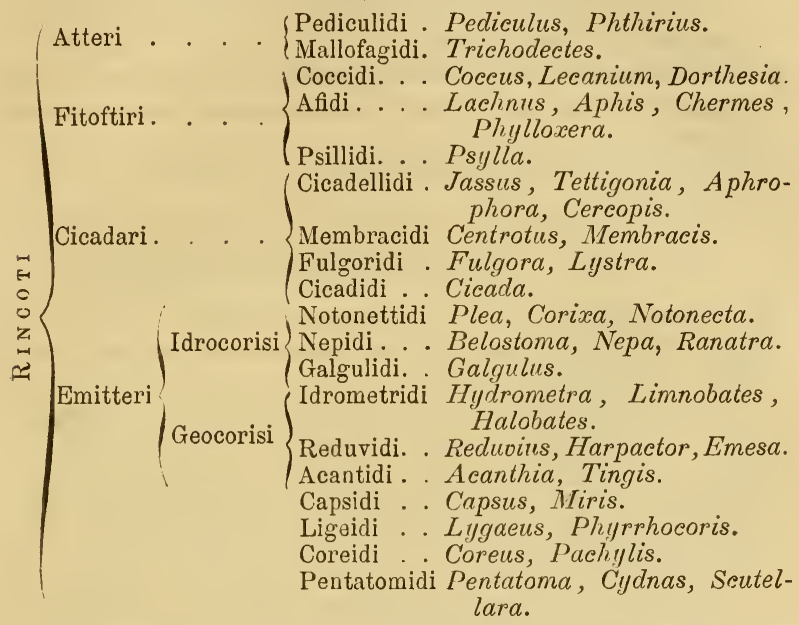

A. Le ali mancano nei due sessi: apparato boccale con un becon corto, carnoso e retrattile. Vivono parassiti sulla pelle degli animali a sangue caldo (Atteri).

a. Tromba carnosa .

Pediculidi.

$a^{\prime}$. Apparato masticatore con una specie di succiatoio: nessun: tromba carnosa

Mallofagidi.

$A^{\prime}$. Due paia di ali membranose nei maschi: femmine nel massimo numero dei casi attere. Vivono sopra varie specie di piante (Fitoftiri).

a. Zampe posteriori non atte al salto.
b. Femmine apode
Coceidi.
$b^{\prime}$. Femmine provviste di zampe . . . Afidi.
$a^{\prime}$. Zampe posteriori atte al salto . . Psillidi.

$A^{\prime \prime}$. Due paia di ali nei due sessi intieramente membranose o le anteriori coriacee (Cicadari).

a. Antenne di tre articoli (in qualche caso due). 
b. Il protorace ricopre il metatorace e giunge fino allo scudetto: ali superiori coriacee, zampe posteriori allungate. Cicadellidi.

$b^{\prime}$. Il protorace ricopre il torace e per lo più si prolunga più o meno sull'addome portando delle espansioni di forma varia: ali membranose . . . . . Membracidi.

$b^{\prime \prime}$. Capo per lo più molto sviluppato prolungato anteriormente e con appendici variamente foggiate: fronte distinto dal vertice; ali anteriori spesso elegantemente colorate. Fulgoridi. $a^{\prime}$. Antenne di sette articoli . . . . Cicadidi. $A^{\prime \prime \prime}$. Le ali anteriori sono semi-membranose e semi-coriacee, ora si trovano in tutti due i sessi, ora mancano nell'uno o nell'altro (Emitteri).

a. Tarsi con un articolo solo o con due: antenne corte e più o meno nascoste.

b. Zampe posteriori appiattite, rivestite lateralmente di lunghi peli e trasformate in remi: dorso convesso tettiforme:

$b^{\prime}$. Zampe posteriori non trasformate in remi.

Notonettidi.

c. Zampe anteriori molto sviluppate e trasformate in organi di presa . . . . . Nepidi.

$c^{\prime}$. Zampe anteriori di uno sviluppo eguale a quello delle altre, le coscie tuttavia un po' più ingrossate: corpo appiattito: occhi grandi e sporgenti . Galgulidi.

$a^{\prime}$. Tarsi con tre articoli (raramente con due): antenne lunghe ben evidenti e portate dall'animale ripiegate in avanti.

$b$. Corpo allungato, lineare e ricoperto di fini peli : corrono sull'acqua e si trovano anche in alto mare. Idrometridi.

$b^{\prime}$. Corpo più o meno appiattito: vivono o sulle piante, o sugli animali, o nel terreno.

c. Capo libero, sporgente con uno stringimento cervicale: antenne di quattro articoli: tarsi di tre articoli. Reduvidi.

$c^{\prime}$. Capo non molto grande e più o meno incassato nel torace: corpo appiattito: tarsi di due articoli: ali spesso mancanti . . . Acantiadi.

$e^{\prime \prime}$. Capo piccolo, triangolare: antenne di quattro articoli: tarso con tre articoli non distinti . Capsidi.

$e^{\prime \prime \prime}$. Capo incassato nel torace: antenne con quattro articoli: tarsi di tre articoli . . . . Ligeidi.

$e^{\mathrm{IV}}$. Torace con delle espansioni membranose ai lati: antenne di quattro articoli . . . Coreidi.

$e^{\mathrm{v}}$. Antenne di cinque articoli (almeno nella massima parte dei casi): seutellum lunghissimo e che giunge circa fino alla metà delle elitre. . . . Pentatomidi. 
ATTERI.

Gli insetti di questo gruppo, che costituivano insieme a varii Ditteri ed a qualche acaro l'ordine degli Atteri, sono caratterizzati principalmente dalla completa assenza delle ali e dalla presenza di un becco molle e retrattile, qualche volta trasformato in un apparato masticatore poco sviluppato. I segmenti del torace talvolta si fondono insieme e si uniscono con quelli dell'addome in modo che riesce difficile di poterli discernere. Le specie di questo sottordine vivono tutte parassite sopra i vertebrati a sangue caldo; hanno mole piccolissima, colori chiari (sono bianchiccie o giallognole) e presentano per lo più i tarsi terminati da robusti uncini. Le loro uova sono per lo più piriformi. In questi animali a sviluppo rapidissimo (il $\mathrm{Pe}$ diculus capitis dell'uomo compie il suo sviluppo in una diecina di giorni) non vi hanno metamorfosi propriamente dette.

Gli Atteri si nutrono di sangue che succiano essi stessi dalla pelle. I Mallofagi possono nutrirsi oltrechè di sangue anche di peli e di penne avendo un apparato masticatore alquanto sviluppato e nello stesso tempo una sorta di succiatoio.

Il genere Pediculus comprende es-

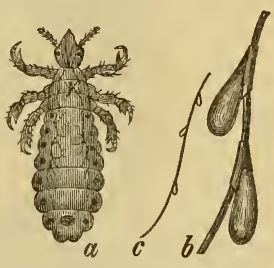

Fig. 57. Pediculus capitis. $c-b$ capelli cun nova. senzialmente due specie, il $P$. capitis che ha l'addome allungato e della stessa larghezza del torace e che vive sul capo dell'uomo deponendo le uova alla base dei capelli, e il $P$. vestimenti che ha la stessa forma del precedente, ma è più grande e vive sul corpo dell'uomo.

Phthirius. Questo genere si distingue facilmente dagli altri pel suo corpo appiattito e largo, con torace piccolo e con un grande addome e per gli uncini robustissimi che terminano i tarsi. Il $P$. pubis vive nella regione del pube e sotto alle ascelle dell'uomo.

Il genere Trichodectes, che appartiene alla famiglia dei Mallofagidi, ha l'aspetto di un Pediculus e vive parassito dei mammiferi e degli uccelli. Le specie conosciute di questo genere sono in grande numero e alcune abitano esclusivamente sul corpo di una determinata specie di uccelli. 


\section{FitoftiRI.}

Questa famiglia comprende specie importantissime sia dal punto di vista della loro applicazione ai varii usi industriali, sia per i danni che arrecano a piante coltivate dall'uomo. Il carattere principale di questo gruppo di Rincoti si è quello di avere due paia di ali membranose, le quali tuttavia mancano frequentemente soprattutto nelle femmine. Spesso nei Fitoftiri si osservano casi di riproduzione partenogenetica.

Nel genere Lecanium Illig. le antenne hanno nove articoli;

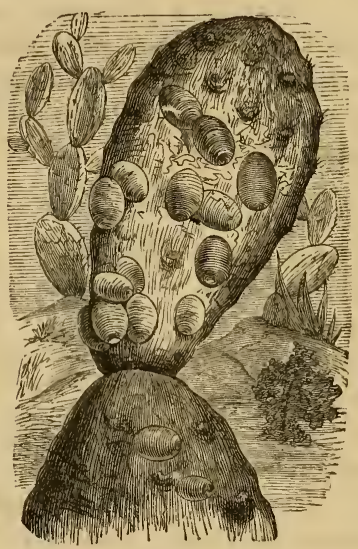

Fig. 58. Coccus lacca.

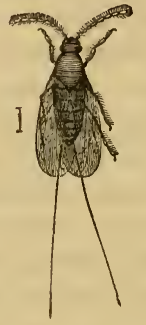

$a$

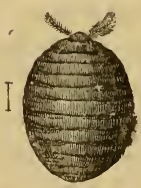

$b$

Fig. 59. Coccus cacti. $a$ maschio $-b$ femmina.

il maschio manca delle ali posteriori e la femmina non presenta una segmentazione distinta. Le specie di questo genere vivono sopra varie sorta di piante; la più celebre di tutte è il L. ilicis Linn., che vive sulla Quaercus coccifera e che fornisce al commercio una sostanza rossa molto pregiata. Menzioniamo anche il $L$. variegatus Olliv., il $L$. persicae Bouch., ecc.

Il genere Monophlebus Leach. merita di essere ricordato per le sue antenne di venti o venticinque articoli. La specie tipica è il $M$. dubius Faler. dell'isola di Giava. Questo genere trovasi tuttavia rappresentato anche in Europa. 
Porphyrophora. Questo genere comprende essenzialmente la $P$. polonica Linn. da cui si ricava quella sostanza colorante che è conosciuta in commercio sotto il nome di cocciniglia di Polonia. Il maschio ha le antenne di nove articoli e porta nella parte superiore dell'addome un grande numero di filamenti.

Affine al genere precedente è il genere Dorthesia Latr., il quale ha antenne filiformi di otto articoli nelle femmine e di nove nei maschi. Le femmine non si fissano e non fanno galle per proteggere le uova e le larve; in ciò esse differiscono da quelle del genere Kermes. La specie tipica è la D. urticae Linn.

Il genere Aleurodes va distinto per avere quattro ali sia nei maschi sia nelle femmine e soprattutto pel suo sviluppo larvale che si scosta alquanto dallo sviluppo consueto dei Rincoti: l'A. chelidonii Lach. è la specie tipica, ed è comune in tutta Europa.

$\checkmark$ AFIDI.

Gli afidi, oltre ai caratteri già accennati, presentano ancora zampe molto lunghe, tarsi a due articoli e inoltre presentano sulla parte dorsale del terzo segmento addominale due cornetti tubifor$\mathrm{mi}$, i quali contengono un liquido dolcigno di cui sono ghiottissime le formiche. Gli Afidi sono celebri per il fatto della riproduzione

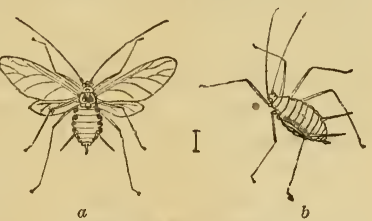

Fig. 60. Aphis rosae. $a$ maschio $-b$ femmina. partenogenetica che in essi si compie nella stagione estiva. Di questa maniera di riproduzione si è già parlato nel cap. XI.

Gli Afidi vivono alle spese dei sughi delle piante e spesso, quando si sviluppano in troppo grande quantità, mandano a male la pianta sulla quale vivono. Questi insetti hanno molti nemici; i principali sono le coccinelle fra i coleotteri che, sia nello stato di larva sia nello stato di insetto perfetto, danno loro accanitamente la caccia.

L'Aphis Linn. è il genere tipico di questo gruppo di Rincoti. Esso è caratterizzato dalle antenne di nove articoli e che sono più lunghe del corpo, dalle zampe relativamente molto lunghe e dalla presenza di due tubetti sull' addome. Numerosissime sono le specie di questo genere e pigliano diversi nomi se- 
condo le piante sopra cui vivono (A. brassicae, $A$. tiliae, ecc.). La specie più nota è l' $A$. rosae.

Meritano ancora di essere menzionati nella famiglia degli Afidi i generi Lachnus e Phylloxera. Il primo è caratterizzato dall'avere antenne con sei articoli e dei tubercoli che sull'addome tengono il posto dei tubetti allungati del genere $A$ phis (1). Il genere Phylloxera poi, divenuto oggigiorno tristamente celebre, ha antenne di tre soli articoli, e comprende forme di mole piccolissima, non ancora molto conosciute. $\mathrm{Cu}$ riosissimi sono i costumi delle specie di questo genere e soprattutto quelli della $P$. vastatrix. Consulti il lettore a questo proposito (2) Signoret: «Phylloxera de la vigne». Ann. Soc.

(1) Ultimamente i signori G. Canestrini e F. Fanzago scopersero e descrissero la metamorfosi di una specie del genere Lachnus, il L. pini negli Atti della Società Veneto-Trentina di scienze naturali, vol. V, 1878.

(2) È enorme il numero degli scritti che in questi ultimi anni venuero pubblicati intorno alla Filossera, e troppo lungo sarebbe il citare anche solo quelli che presentano qualche cosa d'interessante. Malgrado ciò, questi insetti non sono ancora bene noti. Per dare un'idea tuttavia dei costumi della Filossera, io riferisco colle parole stesse del Lichtexstein (Note pour servir à l'histoire des insectes du genre Phylloxera, $2^{\mathrm{e}}$ article. Annales agronomiques, vol. VII. Paris, 1877), la déscrizione del suo ciclo di sviluppo, ciclo, ripeto, che non tutti gli autori ammettono che si compia come vuole il Lichtenstein.

- Cycle normal du phylloxera. Un œuf déposé par une femelle aptère et sans rostre, dont l'abdomen ne contient que cet œuf unique, est placé par elle sous l'écorce des sarments en septembre et octobre.

Cet œuf éclôt du 15 au 30 avril. Il en sort une larve, c'est-à-dire un petit puceron au premier stage de son existence, qui doit changer de peau plusieurs fois et grossir avant d'arriver à son état de perfection.

Ce petit puceron se dirige vers le sommet du sarment de la vigne et s'installe sur la face supérieure des plus tendres feuilles du bourgeon.

Là il pique la feuille. Sous l'effet de cette piqûre les sucs végétaux affluent à l'endroit blessé et forment un bourrelet qui va en s'épaississant autour de l'insecte. Bientôt les lèvres supérieures de ce bourrelet se rapprochent au-dessus même du puceron et finissent par l'englober complètement. Il se trouve au bout de quelques jours dans une galle étroitement ouverte par-dessus, ressortant par-dessous en forme de bourse complètement close, hérissée de petites aspérités et parée quelquefois de vives couleurs rouges.

Pendant que la galle se forme et enveloppe le jeune insecte, il grossit rapidement, change quatre fois de peau dans l'espace d'environ vingt jours, et commence à pondre parthénogénésiquement en remplissant la galle de petits œufsbourgeons dont le nombre difficile à évaluer exactement, peut varier de 200 à 500 . 
Ent. de France, vol. IX, 1869, e vol. X, 1870; Licthenstern : «Notes pour servir à l'histoire des insectes du genre Phyl-

Ces œufs qui ne sont plus des œufs fécondés directement par le sexe mâle, sont ce que j'ai appelé dans mon précédent article des œufs-bourgeons; ils sont analogues, dans le règne animal, à ce que sont les tubercules, bulbes ou rhizomes dans le règne végétal.

Après sept ou huit jours il sort de ces enveloppes d'autres petits pucerons, très-semblables à ceux de la première génération, qui peuvent refaire des galles sur les feuilles, mais qui peuvent aussi très-bien descendre aux racines et vivre alors souterrainement.

Dans ce dernier cas, leur forme varie un peu, leurs antennes s'épaississent, leur peau devient plus rugueuse et même tuberculée, mais leur croissance est à peu près la même; ils changent aussi de peau quatre fois, et après cela se mettent à pondre. Seulement les pontes souterraines sont infiniment moindres que la ponte dans les galles, et au lieu de 200 à 500 œufs que pond la femelle des galles, avant de mourir, celle des racines ne m'a paru pondre que 30 à 40 œufs au plus. La piqûre ne produit pas non plus le même effet sur la racine que sur la feuille, car au lieu de galles il ne se forme que des nodosités sur les radicelles ou de faibles tubercules sur les racines plus fortes. Les nodosités sur les radicelles se forment très-promptement, $j$ 'en ai vu se montrer très-sensiblement en 24 heures.

Les œufs déposés sur les racines éclosent comme ceux des galles au bout de huit jours. Ce sont aussi des œufs-bourgeons pondus parthénogénésiquement sans le concours du mâle.

Les jeunes larves qui en sortent recommencent le même cycle d'existence que leur mère; c'est-à-dire qu'elles muent quatre fois et recommencent à pondre au bout de vingt jour environ.

Seulement, tandis qu'il est assez facile de faire passer le jeune puceron des galles sur les racines, je n'ai jamais pu réussir á faire retourner aux feuilles un puceron nè sous terre. Le puceron aérien peut devenir radicicole dès sa seconde génération (je n'aì pas pu faire l'expérience sur la première génération sortant de l'œuf fécond's), mais il peut aussi rester aérien tant que la vigne pousse de jeunes feuilles; tandis que le puceron devenu souterrain ne peut plus redevenir aérien, ou du moins je n'ai pu réussir à le rendre aérien ou gallicole.

En tout cas l'énorme reproduction aérienne ou gallicole de 200 à 500 œufs, ou la reproduction souterraine plus limitée de 30 à 40 œufs, durent du 15 avril environ jusqu'en novembre (j'ai encore aujourd'hui, 5 décembre, des racines avec des œufs), soit six ou sept générations; on voit d'ici à quel nombre immense on arriverait si tous les phylloxeras se dévelloppaient bien.

A présent, au milieu de ces myriades d'insectes courrant les racines de nos souches, on voit apparaître aux mois de juillet et août, parfois même jusqu'en septembre, une forme d'insecte un peu différente, en ovale allongé, plus grande que ses congénères et qui prend bientôt l'apparence d'une nymphe, nom donné en entomologie à l'état qui précède celui d'insecte ailé ou d'insecte parfait. 
loxera». Annales de la Soc. Ent. Belge, vol. XIX, 1877 e V. Fatio «État de la question Phylloxérique en Europe en 1877,

Cette nymphe porte sur ses flancs deux petits appendices noirâtres qui sont les fourreaux des ailes futures, et après une station de quelques jours sur les radicelles les plus succulentes, elle s'élève à travers les interstices du sol jusqu'à sa surface; là sa peau se fend et l'insecte ailé fait son apparition.

Cet insecte représente la forme que j'ai appelée pupifère. Il n'est par luimême ni mâle ni femelle, mais il porte dans son sein des enveloppes ovoîdes de deux grandeurs différentes.

Ces enveloppes sont ce que je nomme des pupes (nom donné par Latreille aux chrysalides qui sont formées par la peau de la larve se durcissant autour de l'animal), les grandes contiennent des insectes parfaits, sexués femelles, et les petites des mâles.

L'insecte ailé va porter ces pupes dans les vignobles où le vent, ou ses ailes, énormes relativement à sa taille, lui permettent d'arriver; très-souvent on le rencontre pris dans des toiles d'araignée ou jeté sur des feuilles d'arbres ou de plantes qui ne sont pas évidemment faites pour lui, mais en général c'est sous les feuilles de vigne qu'on en rencontre le plus; là il dépose son fardeau. Malgrè tous mes soins, je n'ai pas pu arriver à découvrir si chaque insecte porte le même nombre de pupes, ni si la proportion des sexes est constante; j'ignore même s'il y a des ailés qui ne portent que des femelles et d'autres qui ne portent que des mâles.

Quoi qu'il en soit, les pupes déposées, et dont le cóntenu n'est le premier jour qu'un liquide amorphe comme celui des pupes des diptères, ne tardent pas à montrer un corps plus consistant à travers la fine peau qui les enveloppe. Dès le quatrième ou cinquième jour, la segmentation de l'abdomen, et les yeux se distinguent parfaitement, et bientôt l'insecte parfait s'échappe comme un papillon qui sort de son cocon.

Ce nouveau venu a une forme bien différente des larves produites par l'œuf fécondé ou par l'œuf-bourgeon. D'abord il est à l'état parfait et ne doit pas grossir; il ne saurait du reste prendre aucune nourriture, car les organes buccaux manquent, et à la place du bec robuste des larves, garni de quatre longues soies (deux sont soudées ensemble) on ne voit qu'une faible cicatrice microscopique.

Ensuite il présente des organes génitaux plus développés que dans toutes les autres formes. C'est un pénis triangulaire chez le mâle et une ouverture vulvaire chez la femelle; celle-ci offre de plus par transparence un ouf énorme qui semble la remplir en entier et qui n'attend plus que la fécondation pour devenir le germe d'une nouvelle colonie.

L'accouplement a lieu de suite après l'éclosion. Les mâles très-ardents peuvent féconder plusieurs femelles. Ils meurent bientôt après l'accomplissement de leur fonctions.

La femelle se dirige alors vers le cep et dépose son œuf unique, fécondé, sous l'écorce du sarment; elle-nême meurt à côté.

Cet cuf passe l'hiver et éclôt au mois d'arril, comme je l'ai dit plus haut » 
Genéve 1878 ». Targioni Tozzetri «Del Pidocchio o della Filossera della vite, ecc. ». Bull. della Soc. Ent. Ital., vol. VII, 1875.

Le zampe posteriori foggiate pel salto, le ali sempre presenti nello stato adulto, e le antenne lunghe e di dieci articoli caratterizzano la famiglia degli Psillidi di cui è tipico il genere Psylla Geoff. le di cui specie vivono sopra varie specie di piante producendo delle escrescenze colle loro punture e colla loro saliva sulle foglie e sui fiori. Poche e poco conosciute sono finora le specie dei Psillidi.

CICADARI.

Le antenne sono corte, il capo è grosso, il becco è molto lungo ed è ripiegato al disotto; molte specie hanno i piedi posteriori molto sviluppati ed atti al salto. Molte specie hanno forme stranissime e colori vivaci ed eleganti. La maggior parte delle specie vive sulle piante di cui succiano i sughi nutrizii.

Numerosissimi sono i generi e le specie che appartengono a questo sottordine e vennero raggruppati in varie famiglie come già abbiamo visto. Io citerò solo i generi più spiccati e più importanti.

- Jassus. Capo grosso, corto, ocelli poco spiccati e collocati in una depressione che è al davanti degli occhi, zampe, posteriori fornite di acute spine. La specie tipica di questó genere ricchissimo di specie europee è il $J$. atomarius Fabr., lungo circa m. 0,006, di color bruno variegato di nero e colle nervature delle ali bianche.

Le specie del genere ${ }^{3}$ Ledra Fabr. si riconoscono facilmente pel torace che presenta lateralmente due appendici auricolari e per le tibie posteriori che sono allargate, ed esternamente alquanto appiattite e dentellate. La specie più nota è $I^{\square} L$. aurita Linn.

Ricchissimo di specie dai colori vivaci ed eleganti e sparse per tutto il globo è il genere Tettigonia che ha il capo arrotondato, e più o meno triangolare, gli occelli collocati fra gli occhi, e le zampe cigliate e spinose. Specie di questo genere nota da molto tempo è la ${ }^{3} T$. fasciata Linn. ${ }^{J}$ (Cicada fasciata Linn.), comunissima nel Brasile, di color rosso vermiglio con grosse e ben spiccate fascie nere.

o Aphrophora. Fronte vescicolare, protorace angoloso, tibie 
posteriori con tre grosse spine. La specie tipica è la $A$. spumaria Linn., comune in tutta Europa, e di color grigio cenerino con delle striscie oblique sulle ali. Le larve preparano ed emettono una sostanza bianca spumosa nella quale si av-

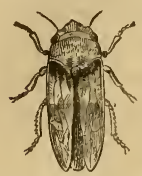

Fo 61 .

A phrophora spumaria. volgono nascondendosi cosi ai loro nemici. Vivono sui salici e possono fare salti molto alti. Affine per molti rispetti al precedente è il genere Cercopis di cui una specie la $C$. sanguinolenta Linn., di color nero con eleganti macchie rosse, non è rara nell'Europa meridionale.

I generi Centrotus, Membracis, Smilia, Ada, Cyphodes, ecc., appartengono alla famiglia dei Membracidi e presentano i caratteri generali di cui abbiamo già fatto cenno. Le specie di questi generi, salvo poche che vivono nel continente antico, appartengono principalmente all'America Meridionale. Fra noi non è raro il Centrotus cornutus, che ha il protorace rialzato e prolungato posteriormente a mo' di spina e presentante lateralmente due prolungamenti auricolari.

o Fulgora. Il capo molto grosso e prolungato anteriormente,

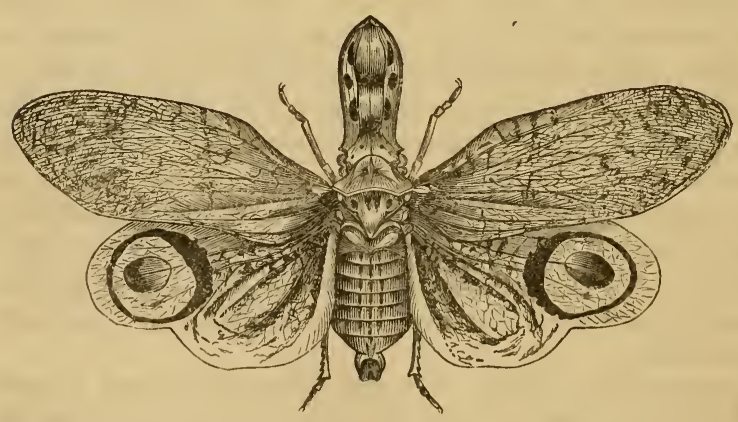

$\checkmark$ Fig. 62. Fulgora lanternaria.

$>$

i colori vivaci, ed i disegni eleganti delle ali caratterizzano questo genere quasi esclusivamente americano. Gli occhi inoltre, cosa non frequente negli insetti, hanno de faccette emisferiche. La specie più anticamente nota è la $\mathrm{F}$. candelaria 
Linn. (1). Non rara fra noi è la ${ }^{\circ} F$. europaea, piccolo insetto di color verde chiaro.

Il genere ${ }^{\circ}$ ystra poi merita di essere ricordato per le proprietà che presenta l'addome delle sue śpecie di produrre abbondantemente la cera (2).

Il ćapo'grosso e largo, il corpo grosso e tozzo, le antenne corte e di sette articoli, l'addome molto grosso, le zampe anteriori spinose e l'apparato del suono caratterizzano il genere Cicada Linn., ed in generale tutti i Cicadidi.

Le cicale sono in generale di mole relativamente grande, e vivono sopra varie specie di piante, nutrendosi di sughi che succiano dalle piante stesse. La loro puntura spesso fa colare da certe piante un umore zuccherino speciale, il quale viene raccolto e messo in commercio col nome di manna: è nota a tutti per questo rispetto la Cicada orni Esch. di Sicilia. Le femmine delle cicale, noterò ancora, sono provviste di un ovopositore fatto a mo' di sega ben sviluppato (3). Sono comuni fra noi le C. fraxini Fabr. di color nero superiormente e giallo-grigiastro Fig. 63. Cicada orni. inferiormente, e lá C. orni Linn. di color giallo-verdognolo macchiettato di nero.

\section{EMITTERI.}

In questo sottordine dei Rincoti le ali anteriori sono semimembranose e sono collegate orizzontalmente sul dorso. In qualche caso le femmine sono attere. Gli Emitteri mandano spesso un odore forte, spiacevole e caratteristico; hanno per lo più bei colori, e parte fanno vita nelle acque, parte invece sui vegetali e nelle case stesse dell'uomo. Sono sparsi per tutta la terra.

I generi Plea Corixa e Notonecta caratterizzano la famiglia dei Notonettidi distinti per avere il corpo convesso e

(1) Intorno alla questione della fosforescenza di questa specie vedi i capitoli VI e V.

(2) La quantità di cera prodotta da qualche specie cinese è tale che essa viene raccolta e messa in commercio col nome di cera cinese.

(3) Intorno al canto delle cicale vedi capitolo V. 
tettiforme, la regione ventrale appiattita e ricoperta di peli, le zampe posteriori appiattite, allungate, ricoperte di peli, e foggiate a mo' di remi. Questi insetti nuotano tenendo la parte ventrale rivolta verso l'alto.

Il genere $P$ lea, che comprende specie di piccolissime dimensioni (fra noi è comune la $P$. minutissima Fabr.), si distingue dagli altri per avere $\mathrm{i}$ tarsi terminati da due uncini.

Comunissimi pure fra noi sono i generi Corixa e Notonecta. Quest'ultimo si distingue dall'altro per il grande sviluppo delle zampe posteriori e per la mole maggiore. Le notonette vivono in grande quantità nei nostri stagni e nelle acque di lento corso, e sono fra i primi insetti che popolano le acque al sopraggiungere della buona stagione; sono pure degli ultimi a sparire al venire dell'inverno (1).

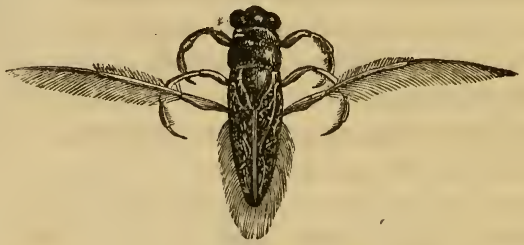

Fig. 64. Notonecta glauca.

Il corpo ora appiattito e largo, ora allungato e stretto, e le zampe anteriori foggiate ad organi di preda caratterizzano la famiglia delle Nepe.

Nepa. Antenne con tre articoli molto corti; corpo appiattito e di forma elittica; un lungo tubo respiratorio; colore bruno-cenerino. Comunissima è nelle nostre acque stagnanti la $N$. cinerea.

Affine al genere Nepa è il genere Belostoma proprio delle regioni Equatoriali dell'America e dell'Asia. Questo genere comprende le forme più grosse dei Rincoti.

(1) Le notonette non sono, a quanto pare, molto sensibili al freddo, ed io stesso ho avuto occasione di osservare ripetutamente delle notonette guizzanti nelle pozze ricoperte da uno strato di ghiaccio anche a mezzo dell'inverno. Si veda anche a questo proposito Michele Lessona * Calendario zoologico in Piemonte». Ann. della R. Accad. di Agric., vol. XVI, 1873. 
Il genere Ranatra è facilmente riconoscibile pel suo corpo allungato e filiforme, per le sue lunge e sottili zampe e pel suo lungo tubo respiratorio. Comunissima è fra noi la $R$. linearis Linn. (1).

Menzioniamo ancora il genere Galgulus che comprende specie americane ed ha il corpo appiattito e largo e gli occhi molto sviluppati. G. oculatus del Brasile e del Messico.

Gli Idrometridi hanno corpo allungato, lineare e ricoperto da fini peluzzi; corrono alla superficie delle acque, per lo più limpide; si nutrono di altri insetti, e le femmine depongono le uova disposte a cordoni allungati sulle piante acquatiche. Ricordiamo il genere Hydrometra Fabr. (Gerris Latr.) provvisto di ocelli e di ali. È comunissima fra noi l' $H$. lacustris L. Il genere Halobates privo di ali

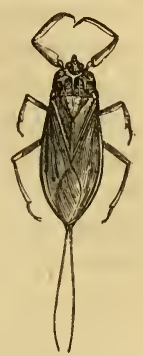

Fig. 65 . Nepa cinerea. e di ocelli, dal corpo corto e tozzo, e colle zampe posteriori e mediane molto lunghe comprende varie specie marine che si trovano anche a grande distanza dalle coste (2).

I Reduvidi vanno distinti per avere un capo libero con un restringimento cervicale; ocelli ben sviluppati; antenne lunghe e sottili di quattro articoli; il torace per lo più spinoso e le zampe anteriori spesso molto sviluppate e foggiate ad organi di presa e

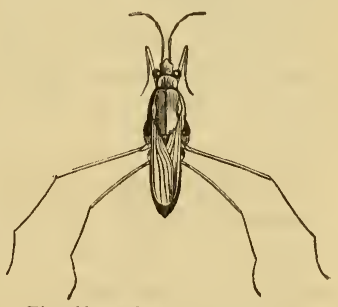

Fig. 66. Hydrometra lacustris. corpo allungato. Molto numerose sono le specie di questa fa-

(1) Nelle pozze del contorno di Torino questa specie si trova principalmente abbondante in principio della primavera e sul finire dell'autunno, nell'estate invece sembra scarsa. Le Ranatre stanno per lo più vicine alla riva, o si raggruppano in grande numero intorno a qualche ramo galleggiante. In qualche annata abbondano tanto che con un sol colpo di rete ben dato se ne possono prendere oltre ad una trentina di individui.

(2) Gigloli "Sulla distribuzione geografica dell'emittero pelagico Halobates ». Bull. Soc. Ent. Ital., vol. II, 1870. 
miglia e sono sparse per tutta la terra; la maggior parte vive sui fiori nutrendosi di altri insetti; qualche specie tuttavia vive nelle case stesse dell'uomo. Tutte le specie pungono assai fortemente e presentano per lo più colori vivaci ed eleganti macchiettature.

Reduvius. La specie più comune di questo genere è il $R$. personatus Linn., lungo circa m. 0,020, la di cui puntura è dolorosissima; è di color bruno nerastro, vive ora nelle case dell'uomo, alle spese della cimice dei letti, ora sotto la scorza degli alberi, nutrendosi di varie specie di altri insetti. La larva di questa specie, cosa degna di nota, si ricopre sempre di polvere affine di sfuggire ai suoi nemici e nello stesso tempo affine di poter sorprendere più facilmente le vittime (vedi capitolo VI).

Emesa Fabr. Carattere principale di questo genere si è quello delle zampe anteriori molto sviluppate e foggiate ad organi di presa. Per questa e per le altre strutture le specie di questo genere hanno molta rassomiglianza colle Mantidi fra gli Ortotteri. Gli insetti di questo genere si trovano nell'America, nell'Africa e nell'Asia Meridionale.

I generi Acanthia, Tingis Syrtis, ecc., caratterizzano la famiglia degli Acanziadi in cui il corpo è appiattito e le antenne contano un numero vario di articoli e le ali talvolta mancano.

A canthia Fabr. (Cimex Linn.), capo appiattito, senza ali, antenne allungate rivestite di piccolissimi peli. È nota a tutti la cimice comune dei letti, che oggigimai è divenuta cosmopolita ed accompagna ovunque (eccetto forse nelle regioni molto fredde e settentrionali o molto elevate sui monti) l'uomo. La cimice dei letti succia il sangue dell'uomo pungendo dolorosamente la pelle. Probabilmente essa è originaria delle Indie Orientali; da quanto vogliono certi autori, l'uomo che vive allo stato selvaggio non sarebbe attaccato dalle cimici. La cosa tuttavia merita di essere meglio studiata. Un'altra specie di Cimice pure parassita dell'uomo è la $A$. ciliata, che si troverebbe, secondo l'Eversmann, nelle case di Casan.

Tingis Fabr. Questo genere comprende un certo numero di specie notevoli per la reticolazione delle elitre e per le espansioni laterali del torace, che vivono sulle piante e sui fiori 
succiando i liquidi delle piante stesse e producendo su di queste colle loro punture varie sorta di escrescenze. Non rara è, ad esempio, la $T$. pyri.

Il genere Syrtis merita di essere ricordato per le sue zampe anteriori foggiate ad organi di presa. Esso comprende numerose specie americane.

I Capsidi comprendono forme piccole ed ornate di bei colori che vivono succiando i liquidi delle piante che nascono nei luoghi umidi e lungo i ruscelli. Il corpo è allungato ed un po' appiattito; le zampe sono lunghe e sottili. Nel genere Capsus (C. trifasciatus Linn.) le antenne terminano a mo' di una mazza. Nel genere Miris le antenne sono allungate ed il capo è prolungato anteriormente. $M$. erraticus Linn.

Ligeidi. I caratteri principali di questa famiglia sono: capo incassato nel torace, antenne filiformi e collocate nella parte inferiore del capo, membrana alare con un numero vario di nervature, tarsi di tre articoli. I Ligeidi comprendono molte specie che si trovano in tutte le regioni della terra, ma che abbondano principalmente in Europa; vivono su varie specie di piante,

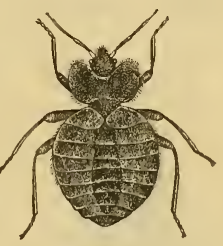

Fig. 67 .

Acanthia lectularia. ed hanno colori e forme spesso eleganti e strane.

Lygaeus. Le specie di questo genere sono numerose assai e presentano per lo più colori vivaci, rosso, nero, giallo, ecc. La specie tipica è il $L$. militaris Fabr., di color rosso-bruno macchiettato di nero.

Il genere Geocoris merita di essere ricordato per la mancanza delle ali posteriori e per l'ingrossamento dell'ultimo articolo delle antenne. G. grylloides (Linn.) del sud dell'Europa.

Il genere Pyrrhocoris Fall. che ha le elitre sprovviste per lo più della membrana, comprende una specie comunissima fra noi, il $P$. apterus Linn., lungo circa $\mathrm{m}$. 0,01 di un bel colore rosso vivo macchiato di nero puro. Si trovano numerosissimi individui di questa specie ai piedi dei muri dei giardini, sotto le scorze ed ai piedi degli alberi, ecc. Esso è uno dei primi insetti che escono fuori al sopraggiungere della buona stagione, ed anzi, come ho osservato ripetutamente nel contorno di Torino, non è raro di vederne, nelle belle giornate 
di inverno, qualche individuo correre sui muri riscaldati dal sole.

Forme molto notevoli presentano i Coreidi, caratterizzati dall'avere le antenne inserite sugli orli del capo e il torace con delle espansioni laterali variamente foggiate.

Coreus. Le specie di questo genere sono sparse per tutta la terra e vivono sopra varie specie di vegetali di cui succiano i liquidi nutrizii. È comune il C. irticollis di color rosso ferruginoso che si trova sulle piante che nascono nei luoghi umidi.

Phyllomorpha. Questo genere è degno di nota per le strane espansioni laterali del torace e dell'addome che lo fanno rassomigliare, come ho gia detto, ad una foglia: P. madagascariensis. Menzionerò pure il genere Neides, non raro fra noi

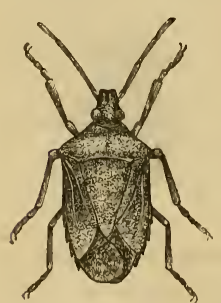

Fig. 68.

Pentatoma grisea. nei luoghi erbosi ed umidi, per la sua rassomiglianza coi ditteri del genere Tipula, $N$. tipularius Linn., ed il genere Anisoscelis che presenta due grosse espansioni membranose nelle zampe posteriori. A. bilineata Fabr. Brasile.

Pentatomidi. Questa famiglia comprende un grandissimo numero di specie divise pure in un grande numero di generi sparsi per tutta la terra, e che vivono come quelle delle famiglie precedenti sopra varie sorta di vegetali, succiando le parti liquide. Le loro zampe sono corte e sottili; lo scudetto invece è molto sviluppato e in qualche caso anzi ricopre quasi totalmente le elitre e l'addome, come si osserva nei generi Tetyra Fabr., e Pachycoris Burm.

Il genere Edessa Fabr., che faceva anticamente parte del genere Pentatoma, è notevole per i prolungamenti spiniformi laterali del torace: E. cervis Fabr., Brasile.

Pentatoma. Capo piccolo; antenne di cinque articoli; addome grosso e largo: tibie ricoperte da peli assai fini. Numerosissime sono le specie di questo genere e sono conosciute volgarmente col nome di cimici di campagna. Le specie europee non hanno in generale colori vivaci, mentre quelle delle regioni equatoriali gareggiano coi coleotteri per la splendidezza delle tinte e dei riflessi metallici. 
Comunissima e nota a tutti pel suo odore caratteristico e spiacevole è la $P$. grisea di color bruno grigio picchiettato di nero che si trova abbondantemente sugli olmi e che, principalmente verso la fine dell'autunno, si incontra spesso anche nelle case delle città.

Scutellera Latr. Carattere principale di questo genere è lo scudetto enormemente sviluppato e che ricopre l'addome e le ali. Le specie di questo genere sono quasi tutte esotiche e presentano in generale colori assai vivaci ed eleganti. S. nobilis Fabr., Indie Orientali.

\section{Bibliografia.}

Oltre alle opere ripetutamente citate intorno agli insetti in generale, si consultino anche gli scritti seguenti:

I. HAHx. - Die wanzenartigen Insecten. Nurnberg, 1831-49.

Амyот et Serville. - Histoire naturelle des Insectes hémiptères. Paris, 1843. Aжroт. - Entomologie française. Rhyncotes. Paris, 1848.

WALKER. - Catalogue of homopterous insects in the collection of the british museum. London, 1850-58.

Fieber. - Die Europäischen Hemipteren nach der analytischen Methode. Wien, 1860. Traduzione francese nella Revue et Magazin de Zoologie, 1875-76.

Flor. - Die Rhynchoten Livlands in systematicher Folge beschreiben. Dorpat, 1860-61.

H. Dexsr. - Monographia Anoplurorum Britanniae. London, 1862.

N. MeLviкow. - Beiträge zur Embryonalentwichlung der Insekten. Archiv.f. Naturg., vol. XXV, 1869.

V. Graber. - Anatomisch. physiol. Studien. über Phthirius inguinalis. Zeitsch. f. wiss. zool., vol. XXII, 1872.

A. Murrat. - Pediculi infesting. the diff. races of man. Edimb., 1861.

F. Rodow. Neue Mallophag. Zeitsch. für die gesam. Naturw., 1869.

Mulsant et Rex. - Histoire naturelle des Punaises de France. Paris, 1865.

Puton. - Synopsis des Hémiptères. Hétéroptères de France. Paris, 1878 (Lygeidi). 
L. R. MeYER. - Verzeichniss der in der Schweiz einheimischen Rynchoten. Solothurn, 1843.

F. Fleber. - Entomologische monographien. Abhandlungen. der. k. böhm. Gesell. der Wissen, vol. III, 1844. Leipzig.

M. Spinola. - Essai sur les Hémiptères. Gènes, 1837.

P. M. Ferrari. - Species Aphididarum hucusque in Liguria lectas, etc. Ann. del Mus. Civ. di St. Nat. di Genova, 1870-72.

- Hemiptera agri-ligustici ecc. Ann. del Mus. Civ. di Stor. Nat. di Genova, 1874.

- Hemiptera Ligustica adjecta et emendata. Ann. del Mus. Civ. di St. Nat. di Genova, 1878. 


\section{XVIII.}

\section{DITTERI}

Insetti con apparato boceale foggiato per succiare e con un solo paio di ali, le anteriori : colle ali posteriori trasformate in due bilancieri : metamorfosi compiute.

L'ordine dei Ditteri (dal greco dıৎ-due e $\pi \tau \in \rho o v-a l a)$ è principalmente caratterizzato dall'avere un solo paio di ali, o, per meglio dire, dall'avere le ali anteriori ben sviluppate e le posteriori rudimentali e trasformate in appendici speciali dette bilancieri. L'ordine dei Ditteri, che comprende un grande numero di forme molto diverse fra loro ed è ricchissimo di specie, venne ammesso da tutti gli Entomologi, ad eccezione di Fabricio, pel quale i Ditteri entravano a far parte dell'ordine degli Autliata.

Il capo nei Ditteri è in generale ben sviluppato, meno in qualche caso come si osserva nei Ditteri infimi (Nycteribia, ecc.). Esso è ben distinto dal torace ed è a questo unito per mezzo di un piccolo penduncolo. Il capo ora è più o meno depresso secondo il diametro antero-posteriore come si osserva nella massima parte dei casi, ora è più o meno arrotondato (genere Pipunculus-Mederetus, Thyreophora, ecc.), ora stretto lateralmente ed allungato (genere Dilophus, Tachydromia, ecc.), ora finalmente presenta due lunghi prolungamenti laterali che portano gli occhi (Diopsis).

Le antenne variano molto e nella loro forma e nel loro sviluppo. In generale esse sono corte e di pochi articoli, talvolta tuttavia si allungano presentandosi ora filiformi, come nel genere Dorthesia, ora con un numero più o meno grande di prolungamenti laterali disposti a mo' delle barbe di una penna (genere Chironomus). Frequentemente le an- 
tenne contano tre soli articoli, e l'ultimo porta un prolungamento stiliforme talvolta diviso in un numero più o meno grande di articoli (Arista). Nei generi Mydas e Cephalocera, le antenne presentano l'ultimo articolo relativamente molto ingrossato. Ora il capo è liscio, ora, come si osserva frequentemente, è ricoperto come il rimanente del corpo di peli più o meno lunghi e più o meno fitti. Nel genere Elaphomia finalmente e nei maschi della Trypeta abrotani il capo ha, al disotto degli occhi, delle espansioni frontali simili alle corna ramificate dei cervi. Si vuole che il capo sia talvolta (Tyreophora) fosforescente.

Gli occhi possono mancare al tutto (genere Nicteribia) od essere variamente sviluppati. Sono relativamente poco sviluppati, ad esempio, nei generi Rhamphomyia, Tachydromyia, Anapera, ecc.). Sono invece molto sviluppati nei generi $\mathrm{Pi}$ punculus, Tabanus, Syrphus, ecc. Nel genere Diopsis, come abbiamo già visto, sono portati da due prolungamenti laterali del capo. Non raramente, soprattutto nei maschi, gli occhi s'incontrano nella linea mediana del capo, e si ha allora un unico grosso occhio. Il capo dei Ditteri porta, in generale, ocelli. L'apparato boccale è foggiato per succiare e presenta, come è già stato detto altrove (capitolo XI), una proboscide formata principalmente dalle mascelle. Lo sviluppo di questa parte della bocca è vario nei Ditteri. La proboscide è corta nella massima parte dei Ditteri. La sua lunghezza è relativamente grande nei generi Bombylius, Phthiria, Usia, ecc., è poi lunghissima (supera parecchie volte la lunghezza di tutto il corpo) nel genere Nemestrina (N. longirostris). I segmenti costituenti il torace si presentano nei Ditteri frequentemente saldati fra loro. Il protorace è cortissimo; è invece molto sviluppato il mesotorace; il metatorace è cortissimo e non raramente si unisce coll'addome. Il torace in quanto alla forma varia assai e segue in generale quella dell'addome. Esso è allungato nei generi Anopheles, Medeterus, Myclas, ecc., globuloso, nei generi Mycetobia, Dilophus, ecc., allargato e depresso nel genere Acanthomera, piriforme nel genere Diopsis, triangolare nel genere Conops, ecc. Ora è liscio, ora è ricoperto di peli più o meno folti o più o meno lunghi.

Il numero dei segmenti che compongono l'addome varia da 
cinque a nove, alcuni dei quali sono spesso più o meno intimamente saldati fra loro. Svariatissima eziandio è la forma dell'addome. Lo troviamo allungato e cilindrico nei generi Anopheles, Corethra, Mydas, ovale nei generi Limnobia, Ctenophora, Dolichopus, ecc., conico colla parte più sottile posta alla sua parte inferiore, nei generi Rhyphus, Leptis, Dolichopus, piriforme, a mo' di gocciola colla parte più stretta posta nel suo punto di attacco col torace, nei generi : Platyura, Ommatius, Diopsis, Calobata, ecc., piriforme colla parte più larga al punto di attacco col torace, nei generi: Estrus Edemagena Tephritis, Phora, ecc., molto grande, più o meno depresso e quadrato od arrotondato, nei generi: Tabanus, Pangonia, Acanthomera, Cuterebra, Echynomyia, ecc., più o meno bilobo nei generi: Ornithomyia, Hyppobosca, ecc.

L'addome finalmente, come il torace, puó essere privo di peli o ricoperto da fitti peli.

Le zampe sono per lo più sottili e spesso molto lunghe (in tutte le Tipule), ora prive di peli, ora finamente pelose. Negli Afanitteri le posteriori sono atte al salto. Nel genere Hoplistomera i femori sono molto ingrossati e dentati nella loro parte interna.

I tarsi presentano cinque articoli e sono terminati da uncini più o meno robusti, e da organi speciali, i quali funzionando molto spesso come ventose, concedono all'animale di poter camminare anche sopra pareti verticali perfettamente liscie.

Delle ali, come ho già detto, sono solamente sviluppate le anteriori, le posteriori essendo trasformate nei bilancieri. Le anteriori sono in generale ben sviluppate, essendo quasi tutti i Ditteri buon volatori.

Le ali sono nude, per lo più trasparenti o variamente colorate. Esaminando il loro contorno inferiore ed interno noi vediamo che esso è, verso la parte più vicina al corpo, intaccato più 0 meno profondamente in modo che pare l'ala sia formata dalla unione di due ali, l'una grande (lobo esterno o alula), l'altra piccola (lobo interno o squama).

Le nervature che solcano l'ala dei Ditteri non sono molto numerose ed inoltre sono quasi tutte longitudinali; scarse sono le nervature trasversali e quindi poche le cellule formate dal- 
l'incontrarsi delle une e delle altre. La disposizione delle nervature ha molta importanza nella classificazione delle famiglie e dei generi dei Ditteri.

I Ditteri sono spesso ornati di colori assai belli, come ad esempio molte specie dei generi Syrphus, Lucilia, Phasia, ecc.

Intorno alla struttura interna ricorderò la presenza di uno stomaco succiatore ben spiccato e di quattro canali Malpighiani (capitolo XI).

I Ditteri hanno metamorfosi compiute. Essi partoriscono o uova, come si osserva nella maggior parte dei casi, o piccole larve che si sviluppano nel corpo della madre (2) (sono in questo caso le specie del genere Sarcophaga), o ninfe come in tutti i Ditteri pupipari.

Le larve sono generalmente apode; rari sono i casi in cui vi siano piccole appendici che facilitino il camminare. Molto frequentemente sono prive di occhi; il loro apparato boccale è destinato ora a masticare ora a succiare. Il Brauer divide le larve dei Ditteri in due sezioni: $1^{\circ}$ Cyclorapha, che comprende le larve acefale e la di cui pelle si rompe secondo una linea curva (Muscaridi, Pupipari); $2^{\circ}$ Orthorapha, comprendente le larve che hanno capo bẹn sviluppato o rudimentale, la di cui pelle si rompe secondo una linea retta (Tanistomi e Nematoceri).

Due sono le maniere che le larve dei Ditteri seguono per trasformarsi in ninfe. Nella massima parte dei casi non v'ha alcuna muta della pelle; la pelle della larva si indurisce, si raggrinza e forma l'involucro esterno duro, nel quale l'insetto passa lo stadio di ninfa. In altri casi la trasformazione ha luogo per un semplice cambiamento della pelle della larva.

I Ditteri sono molto numerosi in specie (e queste spesso si sviluppano in un grandissimo numero di individui), e sono sparsi per tutta la terra ed in tutti i climi, abitando tanto il piano quanto le regioni più elevate dei monti. Una parte si nutre di sostanze vegetali, un'altra parte invece di sostanze animali in decomposizione. Sotto questo ultimo punto di vista ci arrecano una qualche utilità, facendo scomparire molte sostanze organiche in decomposizione. Qualche specie di Ditteri essendo parassita di altri insetti (Lepidotteri), ci è di qualche utilità frenando lo svilupparsi di qualche specie dannosa. Questa 
utilità tuttavia è controbilanciata dai danni gravissimi che molte specie di Ditteri arrecano a varie piante coltivate dall'uomo, e a vari animali di cui l'uomo si serve pe' suoi bisogni, ed infine perchè qualche specie vive addirittura alle spese dell'uomo stesso. Basterà che io ricordi a questo proposito la Mosca degli olivi e la Mosca Tse-tsé dell'Africa centrale, e la comunissima Pulce.

Molte migliaia sonovi, al giorno d'oggi, di specie di Ditteri conosciute. Il loro numero va tuttavia sempre crescendo e molto probabilmente eguaglierà quello degli altri ordini di insetti che sono, per essere stati più ricercati e studiati, al giorno d'oggi più ricchi di specie.

Tavola di classificazione dei Ditteri.

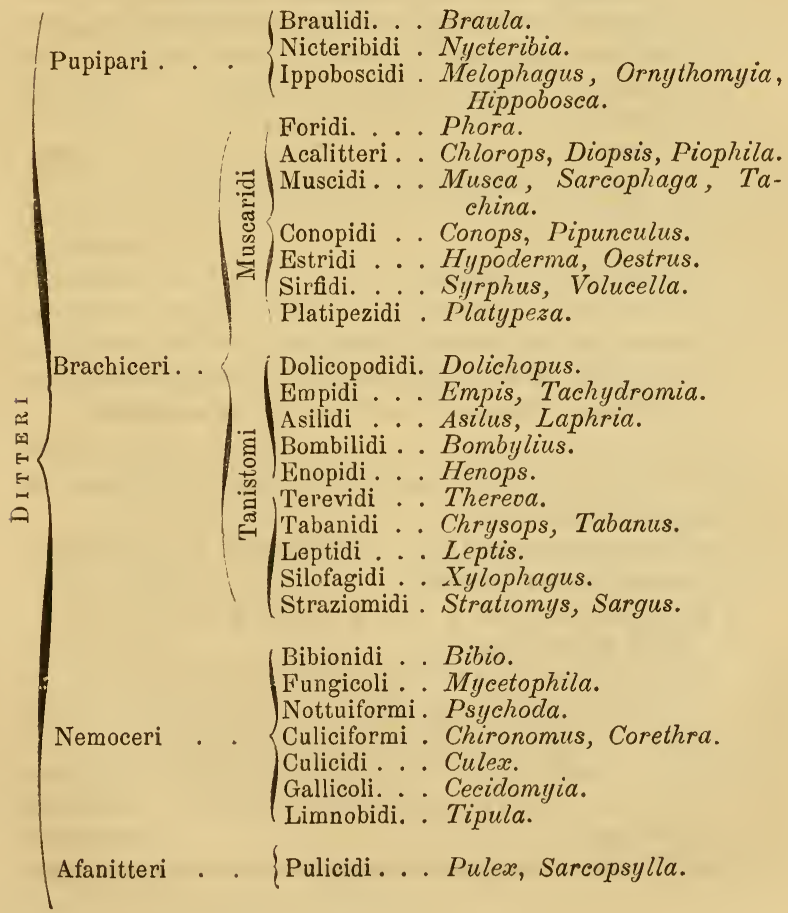


$A$. Corpo depresso e più o meno cilindrico ed allungato.

a. Ali rudimentali o mancanti al tutto: antenne corte e spesso di due soli articoli. Le larve si sviluppano in una dilatazione speciale della vagina (Pupipari).

$\alpha$. Senza occhi e senza ali. Addome con cinque segmenti.

Braulidi.

$\alpha^{\prime}$. Occlıi rudimentali o mancanti, senza àli. Addome di sei segmenti . . . . . . . Nicteribidi. $\alpha^{\prime \prime}$. Occhi grandi . . . . . . Ippoboscidi.

$a^{\prime}$. Ali ben sviluppate: antenne di un numero vario di articoli : le larve subiscono le loro trasformazioni nella pelle stessa di larva che costituisce l'involucro esterno della ninfa (Brachiceri). $b$. Proboscide col lobo terminale per lo più carnoso: mascellein generale atrofizzate (Muscaridi).

a. Antenne di tre articoli: addome di sei segmenti. Foridi.

$a^{\prime}$. Manca la sutura trasversale alla punta dell'ala e la prima nervatura marginale posteriore va direttamente in linea retta fino al margine: bilancieri liberi . Acalitteri. $a^{\prime \prime}$. Porzione terminale della proboscide molle: bilancieri nascosti.... . Muscidi. $a^{\prime \prime \prime}$. Porzione terminale della proboscide formata da lamine chitinose: bilancieri scoperti. Le larve vivono parassite di altri insetti . . . . . Conopidi. $a^{\mathrm{Iv}}$. Proboscide atrofizzata . . . . Estridi. $a^{\vee}$. Ali spesso ornate di colori vivaci: palpi di un solo articolo: addome di cinque articoli. . Sirfidi. $a^{\mathrm{v}}$. Addome di sei articoli: tarsi delle zampe posteriori generalmente ingrossati: corpo per lo più depresso.

Platipezidi.

$b^{\prime}$. Proboscide allungata: mascelle stiliformi più o meno sviluppate (Tanistomi).

a. Proboscide corta e carnosa: addome con cinque segmenti: zampe lunghe e sottili

Dolicopodidi. $a^{\prime}$. Proboscide lunga e dura, provvista di pungiglione: addome di otto segmenti . . . . Empidi.

$a^{\prime \prime}$. Proboscide corta colla parte inferiore dura: un solo pungiglione: mascelle foggiate a mo' di coltello: corpo allungato: addome di otto segmenti, occhi grandi. Asilidi.

$a^{\prime \prime \prime}$. Proboscide lunga e dura: mascelle sottili ed allungate: addome di sette segmenti: corpo depresso e ricoperto da folti peli

Bombilidi. $a^{\text {Iv }}$. Proboscide variamente sviluppata: addome di cinque seg- 
menti: eapo piccolo: occhi grandi: bilancieri nascosti.

Enopidi.

$a^{\vee}$. Proboscide corta colla parte inferiore molle: antenne corte e terminate da un prolungamento stiliforme: addome con sette od otto segmenti . . . Terevidi. $a^{\mathrm{vI}}$. Proboscide corta orizzontale con quattro o sei stiletti nei maschi : occhi riuniti nei maschi : corpo largo e un po' depresso: addome di otto segmenti . Tabanidi. $a^{\text {vu. }}$ Proboscide corta terminata da una porzione molle e da pungiglioni allungati, liberi: addome di otto segmenti: larve con due corti tubi anali . . . Leptidi.

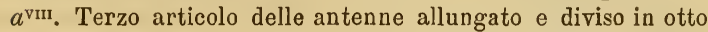
anelli : addome di sette od otto segmenti. Silofagidi. $a^{\mathrm{Ix}}$. Proboscide terminata da un labro ingrossato, molle e retrattile: ultimo articolo delle antenne allungato e diviso in cinque o più anelli: addome di cinque segmenti ed appiattivi. Le larve vivono nell'acqua . Straziomidi. $a^{\prime \prime}$. Ali ben sviluppate, ora nude, ora pelose: le larve mutano la pelle prima di trasformarsi in ninfa.

a. Antenne di un numero di articoli variabile da sei ad undici : addome di sette segmenti: ocelli tutti della stessa grandezza fra loro: aspetto generale del corpo che ricorda quello delle mosche

Bibionidi.

$a^{\prime}$. Antenne filiformi di sedici articoli: addome di sette segmenti, ocelli disuguali fra loro . . . Fungicoli.

$a^{\prime \prime}$. Antenne di quattordici o sedici articoli: corpo molto peloso: ali prive di nervature trasversali : l'aspetto generale del corpo ricorda quello delle Nottue fra i Lepidotteri.

Nottuiformi.

$a^{\prime \prime \prime}$. Antenne del maschio con numerosi prolungamenti laterali disposti in modo da costituire un pennacchio: palpi di quattro articoli : proboscide corta e molle . . Culiciformi. $a^{\mathrm{iv}}$. Antenne dei maschi fatte a pennacchio: proboscide lunga e dura con quattro pungiglioni: palpi di cinque articoli.

Culicidi.

$a^{\mathrm{v}}$. Antenne moniliformi cigliate: capo non prolungato anteriormente

Gallicoli.

$a^{\text {vi }}$. Antenne filiformi: capo prolungato anteriormente: zampe lunghe e sottili: addome di otto segmenti . Limnobidi. $A^{\prime}$. Corpo compresso lateralmente . . . Pulicidi.

\section{PUPIPARI.}

Questo sottordine è costituito dalle forme meno perfette dei Ditteri che sono tutte parassite. 
Il genere Braula (B. coeca Nitzsch) della famiglia dei Braulidi è parassita di vari Imenotteri, soprattutto del genere Bombus.

Le Nitteribie (genere Nycteribia Latr.) sono parassite dei pipistrelli.

Fra gli Ippoboscidi il genere Melophagus Latr., dal corpo privo di ali, è parassita dei Montoni ( $M$. ovinus Linn.): il genere Anapera Meig. dalle ali corte e strette e privo di ocelli vive sulle rondini; il genere Ornythomya Latr. vive sopra varie specie di mammiferi e di uccelli, e finalmente il genere Hippobosca Latr. vive a spese dei cavalli (H. Equina, Linn.).

\section{BRACHICERI.}

Numerosissime sono le specie conosciute al giorno d'oggi e sparse per tutta la terra di questo gruppo di Ditteri. I caratteri principali, oltre quelli già accennati, sono presentati dalle larve le quali vivono nelle sostanze organiche in decomposizione; hanno l'aspetto di un verme allungato; sono provviste spesso di due mascelle uncinate e compiono la loro metamorfosi nella pelle stessa della larva.

Menzionerò fra i numerosissimi generi che formano il sottordine dei Brachiceri i seguenti :

Phora Latr. Le zampe sono molto ingrossate e robuste; le larve vivono negli alveari. Ph. incrassata Meig.: Trypeta Meig. dal capo grande e semicircolare. Le larve vivono sopra diverse sorta di piante. T. cardui Linn., T. signata Meig., ecc. Diopsis Linn., il quale presenta, come ho già fatto ripetutamente notare, il capo che si prolunga lateralmente in due appendici che portano gli occhi: Scatophaga Latr. le di cui specie vivono principalmente sul letame, S. Stercoraria L., Piophila di cui una specie la $P$. casei Linn. vive allo stato di larva sul formaggio.

Noto a tutti è il genere Musca Linn. di cui varie specie vivono in un grande numero di individui nelle case dell'uomo. Il genere Musca comprende specie sovente ornate di colori assai vivaci ed eleganti, e aventi occhi grandi e uniti fra loro nei maschi. Le larve vivono sulle sostanze animali in via di decomposizione.

Sono specie comunissime fra noi la $M$. domestica Linn., 
la $M$. (Lucilia) Caesar Linn. distinta pel suo corpo di un bel color verde metallico, talvolta presentante dei bei riflessi color rame; la $M$. (Calliphora) vomitoria Linn. che ha l'addome di un color azzurro metallico.

Il genere Sarcophaga Meig. è affine al precedente, dal quale tuttavia si distingue per avere gli occhi separati in tutti due i sessi e per essere vivipara. La specie più comune fra noi è la $S$. carnaria Linn. dal corpo bianchiccio solcato da striscie bruno-nere.

Una speciale menzione poi merita il genere Tachina Meig. in quanto che molte delle sue specie sono parassite di molti bruchi. T. grossa Linn., $T$. larvarum Linn., ecc.; di una grandissima importanza sono pure le specie

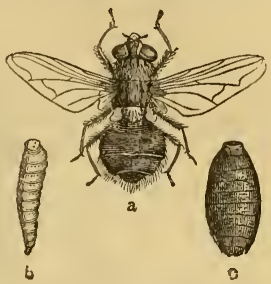

Fig. 69. Riusca vumiloria. $a$ insetto perfetto - $b$ larva $c$ ninfa. del genere Lucilia parassite di varie specie di Batraci anuri(1), ed in qualche caso anche dell'uomo stesso. Si sono osservati casi in cui le larve di una specie di questo genere (L. hominivorax) si sono sviluppate nei seni frontali e nelle fosse nasali dell'uomo, producendo la morte.

I generi Conops Linn., Myopa Fabr., Stomoxys, Pipunculus, ecc., sono molto importanti perchè le loro larve vivono parassite nell'addome di altri insetti (Imenotteri, Ortotteri, Rincoti).

La famiglia degli Estridi comprende ge- $\begin{gathered}a \text { insetto perfetto } \\ b \text { larva }-c \text { ninfa. }\end{gathered}$ neri i quali vivono, allo stato di larva, parassiti o nella cavità frontale o sotto alla pelle o nello stomaco stesso di vari mammiferi. Le larve sono caratterizzate dall'avere parecchi anelli di uncini sul corpo.

Le specie del genere Hypoderma vivono allo stato di larva

(1) Si consulti a questo proposito KrEFFT: "Notes on the metamorphosis of a Dipterous insect of the genus Batrachomya, the larva of which is $\mathrm{Pa}$ rasitical upon various species of Australians Frogs ». Transct. of the Entomol. Soc. of Neu. South-Waies, I, 1864. 
sotto la pelle principalmente dei mammiferi ruminanti. $(H$. bovis Linn., $H$. acteon Br.).

Il genere Cuterebra ha specie che vivono sui rosicanti, sui felini, sui ruminanti ed anche sull'uomo stesso nell'America del sud.

Estrus Linn. Le larve di questo genere provviste di un-

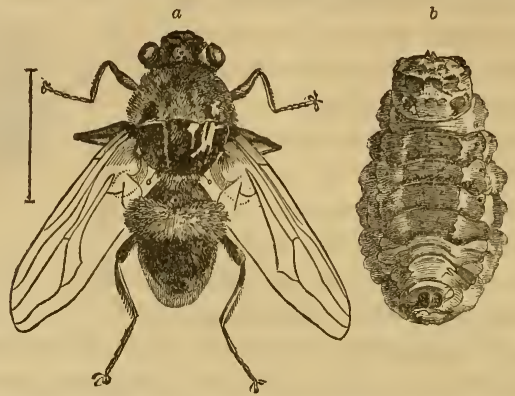

Fig. 71. Hypoderma bovis $-a$ insetto perfetto $-b$ larva.

cini boccali si sviluppano nelle narici dei cervi. Il genere $\mathrm{Ce}$ phalomia è affine al genere CEstrus, ed.una specie la C. ovis Linn. si sviluppa nei seni frontali delle pecore producendo in
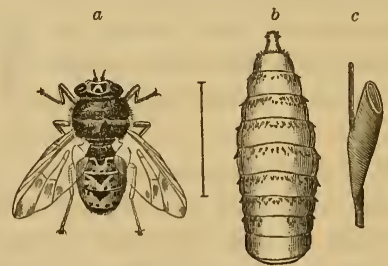

Fig. 72. Gastrus equi $-a$ insetto perfetto $-b$ larva $-c$ ninfa.

queste delle malattie speciali (1), e soprattutto il cosi detto capostorno.

Il genere Gastrus Meig. comprende la specie più nota di

(1) Si consultino a questo proposito i manuali di zoologia medica e veterinaria. Gervais et Van Beneden, Zoologie médicale, Paris 1859. Hercolani, Elementi teorico pratici di medicina veterinaria, Bologna 1861. 
questo gruppo, il G. equi che depone le uova principalmente sulle zampe anteriori dei cavalli. Queste uova vengono dal cavallo stesso, leccandosi, portate nella bocca ed ingoiate. Nello stomaco le larve si sviluppano e si fissano per mezzo degli uncini di cui sono fornite alle pareti stesse dell'intestino. Quando il loro sviluppo larvale è quasi al tutto finito, si staccano e vengono emesse colle feci. Appena uscite dal canal digerente si cacciano sotto terra dove passano lo stato di ninfa.

Il genere Syrphus, le di cui larve si nutrono di gorgoglioni, comprende parecchie specie dall'addome appiattito e dalle zampe sottili ed allungate. S. ribesii Linn., ecc.

Le larve del genere Volucella, distinto per avere un addome largo e cordiforme, vivono nei nidi di varie sorta di imenotteri. $V$. bombylans Linn., ecc.

Il genere Ringia merita di essere menzionato pel grande sviluppo della proboscide. $R$. rostrata Linn.

Il genere Eristalis poi è notevole per la vita acquatica delle sue larve provviste perciò di tubi respiratori. E.'tenax Linn.

I Platipesidi sono caratterizzati dall'avere un corpo appiattito: le zampe corte e le larve che vivono in varie specie di funghi. $P$. boletina Fall.

Assai numerosi sono i generi che costituiscono il gruppo dei Brachiceri Tanistomi : io mi limiterò a menzionare $\mathrm{i}$ principali e più importanti.

Il genere Empis Linn. presenta, come tutti gli. Empidi, un capo piccolo, sferico ed una proboscide relativamente lunga. Le zampe sono ben sviluppate e forti; l'addome è più stretto del torace. Il loro nutrimento consiste in piccoli insetti e nel nettare dei fiori. Pare che le femmine siano più carnivore dei maschi. Le larve vivono nella terra. E. tesselata Fabr.

Il genere Tachydromia Meig. è simile al precedente. Esso se ne distingue tuttavia per avere le zampe mediane e posteriori robuste e dentate.

Il genere Hemesodromia Meig. è notevole per le zampe anteriori molto sviluppate e trasformate in organi di presa. H. mantispa Fabr.

Nel genere Hybos Meig. il capo è piccolo e sferico: la proboscide è corta ed orizzontale : il torace convesso: l'addome, sottile e alquanto ingrossato verso l'estremità inferiore: le 
zampe posteriori sono ingrossate e spesso spinose. È specie non rara l'H. muscarius Fabr.

La famiglia degli Asilidi, che puó venire divisa in due sottofamiglie, Dasipogonini e Asilini, è caratterizzata dall'avere il corpo allungato, l'addome cilindrico, una proboscide corta e le larve che vivono nelle radici e nelle altre parti legnose delle piante. Gli insetti perfetti vivono predando altri insetti.

Il genere più conosciuto e più importante è il genere Asilus Linn., che comprende molte specie che vivono alle spese delle larve di altri insetti. Le specie di questo genere, come in generale tutte quelle della famiglia degli Asilidi, si trovano nei luoghi caldi e secchi e volano principalmente quando il sole è più ardente. Qualche specie attacca anche il bestiame.

Il corpo corto, largo e per lo più rivestito da folti peli, caratterizza la famiglia dei Bombilidi. La maggior parte delle loro larve vivono nei nidi delle Api. Gli insetti perfetti si nutrono del nettare dei fiori sui quali volano nelle giornate calde e di bel sole, producendo un ronzio cupo e spiccato.

Il genere Anthrax Scop. ha occhi piccoli e separati nei due sessi. A. morio Fabr. Nel genere Bombylius Linn., gli occhi sono riuniti nei maschi. B. major 'Linn., nero coi piedi gialli; non raro in Europa.

Il genere Nemestrina merita speciale menzione per la proboscide che supera in lunghezza due volte e più la lunghezza del corpo. N. longirostris Wied. del Capo di Buona Speranza.

I generi Henops Meig., Acrocera Meig., Lasia Wied., ecc., comprendono specie che vivono nell'addome di varie sorta di ragni. Nel genere Henops la proboscide è al tutto atrofizzata. H. gibbosus Linn. Nel genere Acrocera la proboscide è rudimentale. A. orbiculus Linn. Nel genere Lasia finalmente la proboscide è molto sviluppata e più lunga del corpo. L. flavitarsis Wied.

Le specie appartenenti al genere Thereva Latr. sono di piccola mole e notevoli spesso per l'aspetto argenteo del loro corpo. Si sviluppano sovente in grande numero e si nutrono di prede vive e del nettare dei fiori. Le uova vengono deposte dalle femmine nel terreno. È specie comune in Europa la $T$. plebeja Linn.

I Tabani dal corpo grande, largo e robusto, dalla probo- 
scide allungata ed accompagnata nei maschi da quattro o sei prolungamenti stiliformi, sono i più audaci e nello stesso tempo più noiosi fra i ditteri. Essi sono avidissimi principalmente del sangue dei mammiferi. Si noti tuttavia che, a quanto pare, è la sola femmina che si nutre di sangue; i maschi succiano invece il nettare dei fiori. Il genere più importante è il genere Tabanus Linn., che comprende specie sparse in tutte le parti del mondo e che attaccano molte specie di grossi mammiferi ed anche l'uomo. È specie nota e comune il $T$. bovinus Linn.

Menzionerò ancora il genere Chrysops Meig. affine al genere Tabanus notevole per gli eleganti riflessi dorati che presentano i suoi occhi. C. coecutiens Linn.

Il genere Leptis Fabr., tipo della famiglia dei Leptidi, è notevole pei costumi di una specie che si trova dell'Europa meridionale, la $L$. vermileo Linn., la quale a somiglianza del formica-

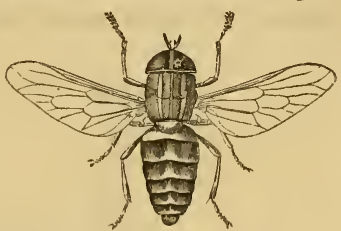

Fig. 73. Tabanus borinus.

leone si scava degli imbuti nella sabbia e tende insidie agli insettucci di cui si nutre.

I Silofagidi comprendono un piccolo numero di specie variabili assai nell'aspetto generale del corpo e viventi nel legno di varie sorta di piante. Il genere Xilophagus Meig. ha lo scutellum privo di spine. $X$. ater Fabr. Il genere Baris Latr. invece presenta lo scutellum orlato di spine. B. clavipes Linn.

Negli Straziomidi il corpo è largo; l'addome è elegante ed arrotondato; le antenne presentano il terzo articolo con 50 6 anelli. Gli Straziomidi vivono sulle foglie o sui fiori di cui succiano il nettare. Le larve compiono la loro metamorfosi nella loro stessa pelle. Ora le larve fanno vita acquatica (Stratiomys), ora vivono nel legno marcio (Pachygaster), ora finalmente vivono nello sterco dei ruminanti (Sargus). Il genere Stratiomys comprende specie dai colori eleganti, che vivono allo stato perfetto sui fiori; le larve di color brunogrigio sono allungate ed hanno gli ultimi segmenti assottigliati a mo' di coda; l'ultimo segmento porta un fascio di peli ri- 
gidi. Lo S. chamaelon L. è comune in primavera sui fiori di biancospino.

Fra le specie dei generi Sargus e Pachygaster ricorderò il S. ater Panz. ed il P. cuprarius Linn.

NeMoceri.

I ditteri di questo sott'ordine, che quantunque sia ricco di specie ne comprende tuttavia un minor numero che non il sottordine precedente, hanno il corpo molle ed allungato; le antenne $o$ filiformi o foggiate a pennacchio; le zampe in generale molto lunghe e sottili; le ali allungate e per lo più strette; i bilancieri sempre liberi; l'addome con un numero di segmenti variabili da sette a nove. Le larve vivono ora nell'acqua, ora nella terra, ora nelle galle, ora nei funghi, ecc.,

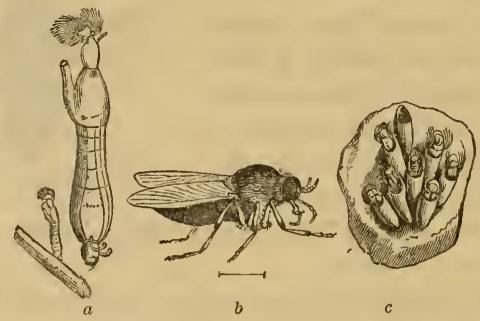

Fig. 74. Simulia maculata.

$a$ larve $-b$ insetto perfetto $-c$ ninfe attaccate ad una pietra.

e presentano un capo ben distinto dal rimanente del corpo, carattere questo di superiorità sopra gli altri ditteri.

I Nemoceri sono sparsi ovunque e spesso si sriluppano in un modo straordinario tanto da formare delle vere nuvole di insetti. Una parte delle specie si nutre del sugo dei fiori, un'altra parte succia il sangue agli animali ed all'uomo stesso. In molte specie hanno luogo varie generazioni nel corso dell'anno.

Il genere Bibio Geoffr. è il tipo della famiglia dei Bibionidi, che hanno l'aspetto delle mosche comuni, e pel complesso dei suoi caratteri fa il passaggio fra i Nemoceri ed i Brachieri. Le specie del genere Bibio vivono sui fiori. B. hortulanus Linn.

Il genere Simulia Meig. è degno di nota in quanto che le femmine succiano, facendo dolorose punture, il sangue dei 
mammiferi ed anche dell'uomo. Abbondano soprattutto nelle regioni meridionali umide ed acquitrinose.

I Fungicoli, come dice il nome stesso, comprendono specie che vivono nei funghi. È notevole il genere Sciara di cui le larve prima di trasformarsi in ninfa si riuniscono insieme in modo da formare una lunga catena sinuosa ed imprendono delle emigrazioni, S. thomae Linn., S. flavipes Meig.

I Nottuiformi meritano di essere menzionati per le loro ali pelose e frangiate, e pel complesso della struttura del corpo che ricorda quella della Nottue fra i Lepidotteri. Le larve vivono nelle sostanze vegetali in putrefazione. Psychoda phalaenoides Linn.

La massima parte delle specie appartenenti alla famiglia dei Culiciformi vivono nell'acqua; sono di piccola mole ed hanno le antenne dei maschi foggiate a mo' di pennacchi.

Chironomus Meig. Questo genere comprende un grande nu-

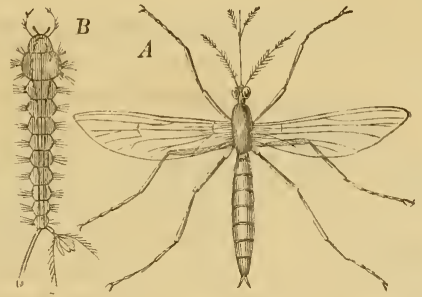

Fig. 75. Culex pipiens $-A$ insetto perfetto $-B$ larva.

mero di specie piccolissime e delicatissime che sono sparse per tutta la terra ed abitano anche le regioni piu fredde circumpolari e le alte regioni alpine. La specie più frequente è il C. plumosus Linn., di cui la larva di color rosso sangue abbonda spesso straordinariamente nelle acque stagnanti e di lento corso. Affine al genere precedente è il genere Corethra Meig., di cui la larva fa pure vita acquatica, C. plumicornis Fabr.

Culex Linn. Questo genere, tipo della famiglia dei Culicini, comprende una specie, il C.pipiens Linn., volgarmente zanzara, nota a tutti per le sue dolorose punture. Le larve delle specie del genere Culex vivono in grandissimo numero nelle acque stagnanti ed abbondano principalmente nei luoghi caldi 
ed umidi. Pare che le femmine sole succino il sangue e che i maschi invece si nutrano del nettare dei fiori.

I Gallicoli comprendono forme piccolissime di ditteri che vivono, come i Cynips, nelle escrescenze che essi producono pungendo le foglie, i fiori o i ramoscelli. Una specie la Cecidomyia destructor Say. riesce, soprattutto nell'America del Nord, dannosissima al grano. Il genere Miastor pure appartenente a questa famiglia presenta, come ho già detto, un caso di riproduzione vivipara nello stadio larvale.

Tipula Linn. Questo genere è caratterizzato dal capo prolungato anteriormente, dalle antenne filiformi e dalla grande lunghezza e sottigliezza delle zampe. Questo genere conta un grande numero di specie. Le larve di molte specie europee vivono sulle radici di varie piante e riescono talvolta dannose alle piante stesse. Sono specie comuni la $T$. oleracea Linn., la $T$. gigantea Schrk., ecc.

\section{AFANITTERI.}

Pulex Linn. Il corpo è di forma ovale ed è compresso lateral-
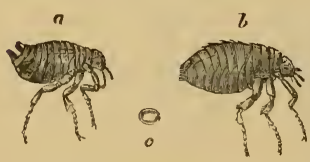

Fig. 76. Pulex irritans. $a$ maschio $-b$ femmina $-c$ uovo. mente; il capo è piccolo; i palpi labiali formano la guaina del succiatoio; le mandibole allungate unitamenté alla linguetta costituiscono il succiatoio; le zampe sono robuste; le posteriori sono più sviluppate delle altre ed atte al salto; l'addome è molto grande; le ali mancano. Le larve sono apode, hanno un capo ben distinto e delle mascelle. La $P$. irritans Linn. di color rosso cupo è parassita dell'uomo; la $P$. canis Dugés vive principalmente sui cani e sui gatti; la $P$. vespertilionis Dugés si trova sui pipistrelli, ecc.

Il genere Sarcopsylla comprende una specie, la $S$. penetrans che abita le regioni sabbiose dell'America meridionale e riesce dannosa all'uomo in quanto che la femmina si introduce sotto la pelle del piede e vi depone le nova. Le larve che poi ne sbucciano danno origine ad ulcere dolorosissime. 


\section{Bibliografia.}

J. W. Meigex. - Systematische Beschreib. der bekan. Europ. zweiflüg. Insec. $1^{a}$ parte. Aachen, 1818-1838. Wiedemann. Aussereurop. zweiflüg Insecten, $2^{\text {a }}$ parte. Hamm, 1828-30.

Macquart. - Hist. nat. des insectes Diptères, Parigi, 1834-35.

H. LoEw. - Dipterologische Beiträge, Berlin, 1845-61.

F. WALKEL. - Insecta britannica. Diptera, 1851-56.

R. Schiner. - Fauna Austriaca, Wien, 1860.

Brauer. - Kurze Charakteristik der dipterenlarven. Verh. der Zool. bot. Gesell. Wien, 1869.

J. O. Westwoop. - On Nycteribia, ecc. Trans. Zool. soc. of London, 1875.

F. BraUer. - Monographie der Oestriden. Wien, 1863.

A. Dugès. - Recherches sur les caractères zoologiques du genre puce. Ann. des Sc. Nat., vol. XXVII, 1832.

Laxdo1s. - Anatomie des Hundeflohes, Dresden, 1867.

Bonnet. - Mémoire sur la puce pénétrante. Ann. $d$. Sc. Nat., $5^{a}$ serie, vol. VIII. L. Bellardi. - Saggio di ditterologia Messicana. Mem. Acad. delle Sc. di Torino, 1859.

Rondanr. - Dipterologiae Italicae prodromus. Parma, 1856-59 e numerosissimi scritti stampati negli Annali della Soc. Ent.di Francia, nel Bol. Ent. Italiano, negli Atti della Sac. Ital. di Milano, nei Nuovi Annali di Sc. Nat. di Bologna, ecc. 


\section{LEPID0TTERI}

Insetti con apparato boccale foggiato per succiare: ali ricoperte da squame rariopinte: metamorfosi compiute.

I Lepidotteri ( $\lambda \in \pi ı \zeta$, squama, $\pi \tau \epsilon \rho o ́ v$, ala) costituiscono uno degli ordini più naturali degli insetti. Il numero delle specie che questo ordine comprende è molto grande e le specie variano molto fra loro nei colori ed anche nella forma delle ali. In tutte si riconosce, tuttaria sempre costantemente, lo stesso piano di struttura. Non sarà quindi necessario spendere molte parole per dare un'idea generale di questo ordine.

Il capo nei Lepidotteri è quasi sempre ben sviluppato e ha una grande mobilità: le antenne ora sono allungate, filiformi e terminate a clava, ora portano delle espansioni lamelliformi disposte a mo' di frangia, o come le barbe di una penna, o riunite a ciuffo all'estremità dell'antenna stessa. Generalmente le antenne sono più sviluppate nei maschi che non nelle femmine. Gli occhi sono quasi sempre ben sviluppati. Gli organi boccali sono foggiati nello stato adulto per succiare (la proboscide può mancare intieramente od essere lunghissima), per masticare invece nello stato larvale. I segmenti del torace sono tutti saldati fra loro. Le zampe sono, relativamente alla mole del corpo, poco sviluppate e ciò è in rapporto col grande sviluppo delle ali: in molti casi le zampe anteriori sono atrofizzate. Sulle zampe si trovano generalmente degli speroni molto sviluppati.

I tarsi hanno cinque articoli. Le ali sono sempre ben sviluppate, qualche volta tuttavia mancano nelle femmine. Ora le 
ali anteriori e le posteriori hanno uno sviluppo ad un dipresso eguale, ora le posteriori sono più piccole delle anteriori, ora invece le posteriori si prolungano inferiormente in una coda più o meno sviluppata. Intorno alle forme ed ai colori mimetici delle ali, ed intorno alla struttura delle squame che le rivestono si vedano i capitoli IV e VI.

Relativamente molto grande è nei Lepidotteri la centralizzazione del sistema nervoso. Nell'insetto allo stato perfetto la catena gangliare presenta due soli gangli toracici e cinque addominali, nello stato larvale invece i gangli sono undici.

I Lepidotteri hanno metamorfosi compiuta. Le larve o bruchi hanno forma allungata e vermiforme, e presentano un numero vario di false zampe. In molti casi le larve tessono un bozzolo nel quale passano lo stadio di ninfa o di crisalide.

Sono stati osservati nei Lepidotteri casi di ermafroditismo e di partenogenesi. I Lepidotteri Ropaloceri presentano frequenti fatti di polimorfismo sessuale. Non sono rari pure i casi di polimorfismo dovuto all'influenza delle stagioni.

I Lepidotteri sono sparsi per tutta la terra; fanno vita terragnola, meno qualche specie che nello stato larvale vive nell'acqua e presenta dei filamenti branchiali, e si nutrono di sostanze vegetali. Molte specie per la loro voracità e pel loro grande svilupparsi arrecano spesso gravi danni all'uomo.

Lo sviluppo di molte specie di Lepidotteri è frenato da un grande numero di Icneumonidi e di Tachinarie che sono parassiti dei bruchi. 
Tavola di elassificazione dei Lepidotteri.

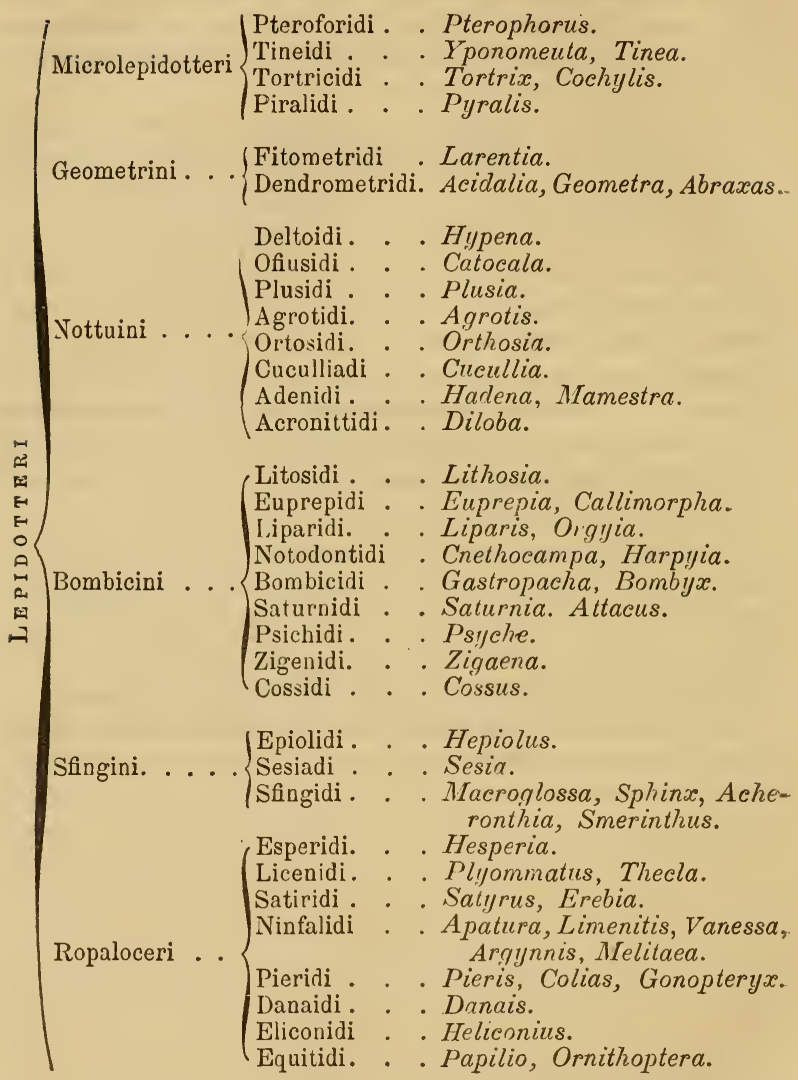

A. Corpo di piccolissime dimensioni : antenne allungate, fliformi : ali anteriori con due e raramente tre nervature dorsali: ali posteriori con tre (qualche volta due) nervature marginali interne. a. Ali divise in lobi con margini rivestiti di peli. Pteroforidi. $a^{\prime}$. Ali non divise a lobi. 
a. Antenne allungate e filiformi: palpi mascellari lunghi.

Tineidi.

$a^{\prime}$. Antenne allungate e filiformi : palpi mascellari atrofizzati. Tortricidi.

$a^{\prime \prime}$. Antenne dei maschi spesso pettinate: palpi mascellari molto sviluppati . . . . . Piralidi.

$A^{\prime}$. Corpo di dimensioni mediocri : allungato: ali grandi : antenne allungate, filiformi e col primo articolo ingrossato.

a. La nervatura dell'ala posteriore piglia origine dalla nervatura mediana anteriore. . . . Fitometridi.

$a^{\prime}$. La nervatura costale delle ali posteriori piglia origine dalla base dell'ala . . . . . Dendrometridi.

$A^{\prime \prime}$. Corpo relativamente allungato ed appuntito posteriormente: antenne spesso pettinate nei maschi: palpi e proboscide ben sviluppati: tibie con grossi speroni . . Nottuini.

a. Di aspetto simile a quello dei Piralidi : ali posteriori con due nervature marginali interne: tibie per lo più lunghe ed inermi . . . . . Deltoidi.

$a^{\prime}$. Aspetto simile a quello dei Geometrini: zampe robuste e con speroni . . . . . . Ofiusidi.

$a^{\prime \prime}$. Addome sottile con ciuffi di peli : ali con spazii più o meno grandi privi di squame. . . . Plusidi.

$a^{\prime \prime \prime}$. Addome conico, senza ciuffi di peli: tibie delle zampe mediane e posteriori con spine più o meno spiccate.

Agrotidi.

$a^{\mathrm{IV}}$. Torace più o meno convesso: tibie delle zampe mediane e posteriori talvolta con spine . . Ortosidi.

$a^{v}$. Addome lungo ed appuntito: ali anteriori lanceolate: tibie inermi

Cuculiadi.

$a^{\mathrm{v}}$. Torace convesso: ali anteriori triangolari. Adenidi.

$a^{\mathrm{vI}}$. Aspetto simile a quello dei Bombicidi : torace arrotondato anteriormente: zampe coperte di peli : tibie inermi.

Acronittidi.

$A^{\prime \prime \prime}$. Corpo ingrossato e tozzo, ricoperto da fitti e lunghi peli : ali allungate e filiformi nella femmina, pettinate invece nei maschi. $a$. Antenne cigliate: occhi privi di peli: proboscide ben sviluppata: ali posteriori larghe con due nervature dorsali.

Litosidi.

$a^{\prime}$. Antenne cigliate (nei maschi talvolta sono pettinate): ali posteriori con due nervature marginali interne. Euprepidi. $a^{\prime \prime}$. Antenne corte e doppiamente pettinate: proboscide poco sviluppata o atrofizzata . . . . Liparidi. 
$a^{\prime \prime \prime}$. Corpo ricoperto da molti peli finissimi : zampe coperte in parte da lunghi peli Notodontidi. $a^{\text {Iv }}$. Antenne pettinate nei due sessi : palpi molto sviluppati: tibie posteriori con speroni terminali non molto sviluppati. Bombicidi.

$a^{\mathrm{v}}$. Antenne dei maschi doppiamente pettinate: zampe corte: le posteriori senza speroni . . . Saturnidi.

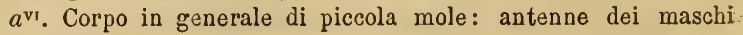
doppiamente pettinate : privi di palpi e di proboscide. Psichidi. $a^{\mathrm{vII}}$. Antenne terminate a clava o dentate: addome allungato: ali anteriori spiccatamente più grandi delle posteriori: proboscide molto sviluppata. . . Zigenidi.

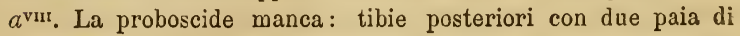
speroni

Cossidi.

$a^{1 x}$. Corpo allungato: antenne corte, nè pettinate, nè dentate. Epiolidi.

$A^{\text {Iv }}$. Corpo grosso, allungato ed appuntito: ali anteriori allungate e strette: posteriori poco sviluppate: allo stato di riposo stanno orizzontali: proboscide allungata: antenne corte ed appuntite.

a. Ali trasparenti e prive quasi intieramente di squame.

Sesiadi.

$a^{\prime}$. Ali intieramente ricoperte da squame . Sfingidi.

$A^{\mathrm{v}}$. Corpo relativamente non molto grosso: allungato: ali molto grandi: antenne terminate a clava: proboscide ben sviluppata: nessun palpo mascellare . . . . Ropaloceri.

a. Lepidotteri di piccole dimensioni : occhi di forma emisferica. e privi di peli . . . . . . Esperidi. $a^{\prime}$. Lepidotteri di piccole dimensioni: occhi di forma ovale. Licenidi.

$a^{\prime \prime}$. Zampe anteriori atrofizzate.

a. Palpi più corti del capo . . . Satiridi.

$a^{\prime}$. Palpi più corti del capo coll'ultimo articolo appuntito.

$a^{\prime \prime}$. Palpi corti e divaricati fra loro . Danaidi.

$a^{\prime \prime \prime}$. Palpi più lunghi del capo . . Eliconidi.

$a^{\prime \prime \prime}$. Zampe anteriori ben sviluppate.

a. Ali arrotondate e col margine intiero: palpi con tre articoli

Pieridi.

$a^{\prime}$. Ali posteriori con dentellature e con prolungamenti codiformi più o meno spiccati: palpi più o meno sviluppati.

Equitidi. 


\section{MicrolePidotTERI.}

I Lepidotteri di questo gruppo, come dice la parola stessa, comprendono le forme più piccole di tutto l'ordine e nello stesso tempo anche le più dannose in varii modi all'uomo. Le antenne sono filiformi ed allungate; le ali anteriori hanno due o tre nervature dorsali; le posteriori hanno tre nervature marginali interne. Le specie che costituiscono questo gruppo sono numerosissime. Ricorderò solamente i generi principali.

Pterophorus. Questo genere, tipo della famiglia dei Pteroforidi, è caratterizzato dall'avere le ali divise in lobi finamente frangiati. Il grado di divisione ed il numero dei lobi varia nelle diverse specie. Nel $P$. pentadactylus L. le ali sono divise in cinque divisioni o lobi. Nella $A l u$ cita hexadactyla ciascuna ala è intieramente divisa in sei lobi. Il Pterophorus pentadactylus è di color bianco; si trova in giugno

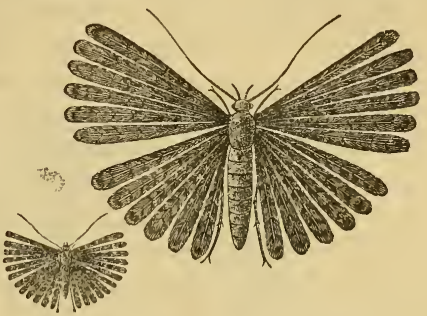

Fig. 77. Alucita exadactyla (di grandezza naturale ed ingrandita). ed in luglio lungo le siepi, volando e di giorno e di sera. Menzionerò pure il $P$. rhododactylus piccola farfallina dalle ali anteriori falcate e divise per un terzo della loro lunghezza in due, ed elegantemente macchiate di bruno, di rosso e di bianco, e dalle ali posteriori divise in tre lobi e di color rosso ferruginoso.

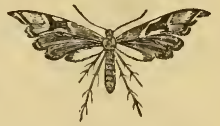

Fig. 78. Pterophorus rhododactylus.

Numerosissimi sono i generi che formano la famiglia dei Tineidi; nella sola Europa se ne contano oltre a cento e settanta. I caratteri generali sono: antenne allungate e filiformi ; palpi labiali molto sviluppati e spesso più lunghi del corpo; ali allungate e orlate di una lunga frangia e per lo più elegantemente colorite e macchiettate. Allo stato di larva intaccano un gran numero di vegetali e di sostanze animali come lane, pelli, ecc.

Menzionerò pure il genere Yponomeuta caratterizzato da palpi non molto lunghi, le di cui specie hanno le larve che vivono riunite in una tela comune fra i rami degli alberi. Sono fre- 
quenti in Europa l'Y. padella L., l' $Y$. cognatella Hub. e l' $Y$. evonymella L. Le specie di questo genere arrecano spesso danni gravissimi ai frutteti. La specie più dannosa è l' $Y$. cognatella (1).

Le numerose specie del genere Nepticula sono piccolissime e si scavano gallerie nello spessore delle foglie.

Il genere Tinea è quello fra tutti i Tineiti che comprende il maggior numero di specie e nello stesso tempo le specie più

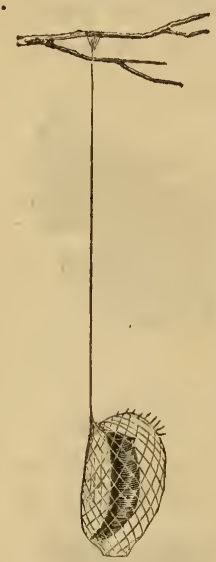

Fig. 79. Bozzolo di tignola del Brasile. dannose. I palpi mascellari sono molto sviluppati; i palpi labiali sono più lunghi del capo; le zampe sono relativamente lunghe e grosse. Le larve delle specie di questo genere che, per la loro voracità e pei danni che esse ci arrecano, vennero con ragione paragonate dal Duponchel, ai topi ed ai sorci, si fabbricano astucci di varia forma ora fissi, ora mobili, ora a pareti piene, ora invece foggiate elegantissimamente a mo' di graticolo e sospese ad un ramo, come si osserva in qualche specie del Brasile (Fig. 79).

Le tignuole possono, come già fece lo Chenu, venire divise, secondo le sostanze che intaccano, nelle tre categorie seguenti:

$1^{\text {a }}$ Specie dannose alle stoffe ed alle pelliccie (Le specie più comuni sono $T$. pellionella Linn., la T. tapezella e la T. cri-

nella Treitsc.);

$2^{a}$ Specie dannose ai cereali (Tinea granella Linn.);

$3^{a}$ Specie fitofaghe dannose a varie sorta di frutti e di vegetali ( $T$. cerasiella Hubn. che vive sul pomo, sul cigliegio, sul pruno, ecc.) (2).

I generi Tortrix, Carpocapsa, Grapholitha, Cochylis, caratterizzano la famiglia dei Tortricidi.

(1) Si è cercato di trar partito della seta che le larve delle specie del genere Yponomeuta producono in abbondanza, e se ne è ottenuto un tessuto assai delicato e sottile.

(2) Si consultino, intorno alle maniere di impedire i danni di questi insetti, le opere di Entomologia applicata menzionate nella Bibliografia del capo II. 
Le specie dei generi sopra nominati vivono o a spese delle parti carnose del frutto o delle foglie di varie sorta di vegetali ai quali non raramente riescono assai dannose.

Il genere Tortrix, che presenta la nervatura mediana delle ali posteriori nuda e gli sproni interni delle tibie posteriori più lunghi che non gli esterni, comprende specie che vivono sulle foglie di varie piante piegando le foglie stesse in modo da formarne dei tubi e dei cartocci di varie forme. Sono specie note la $T$. viridana che vive sulla quercia e la $T$. strobilana che vive sui pini.

Il genere Cochylis merita di essere ricordato per una specie

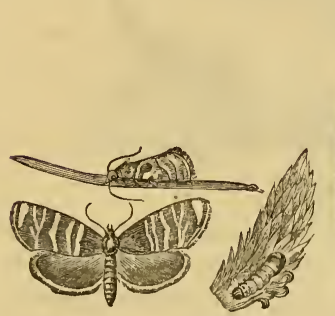

Fig. 80. Tortrix strobilana.

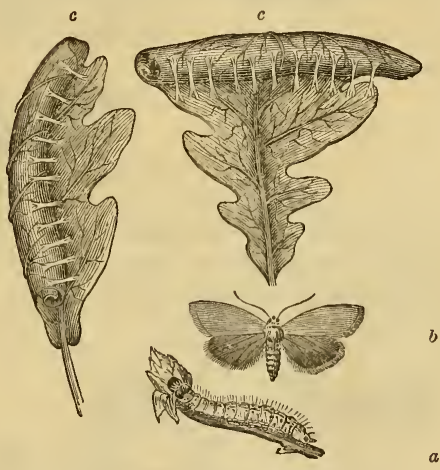

Fig. 81. Tortrix viridana.

$a$ larva $-b$ insetto perfetto $-c$ astucci.

che spesso porta danni grandissimi ai vigneti la $C$. roserana. Questa specie non costruisce astuccio colle foglie; ma invece si porta ad attaccare i fiori e, cominciando a divorarli dal calice, ne distrugge in poco tempo un grande numero. Le larve della seconda generazione attaccano pure gli acini e ne mandano a male una grande quantità.

Le specie del genere Carpocapsa vivono principalmente a spese dei frutti carnosi; la specie tipica è la $C$. pomonana che si trova frequentemente allo stato di larva nell' interno dei pomi e delle pere.

Il genere Grapholitha comprende specie affini a quelle dei generi già citati; le sue larve vivono di foglie, grani, ecc., e 
compiono la loro metamorfosi in un involucro fatto di un fitto tessuto avvolto di terra, G. siliceana, comune in quasi tutta Europa.

Il genere Pyralis è il più importante della famiglia delle Piralidi; la specie più nota è la $P$. vitana o pileriana, pic-

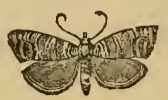

Fig. 82. Carpocapsa pomonana.

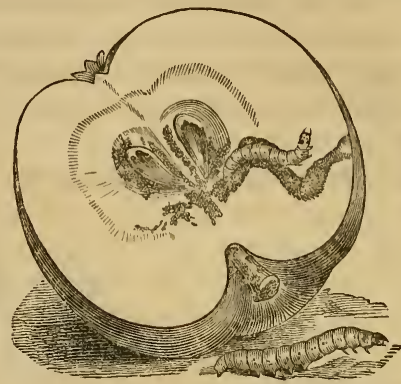

Fig. 83. Carpocapsa pomonana - bruco.

cola farfalla dalle ali gialle con riflessi verdastri e dorati e delle striscie brune più o meno spiccate, che arreca spesso danni gravissimi ai vigneti. Si consulti a questo proposito Audouin,

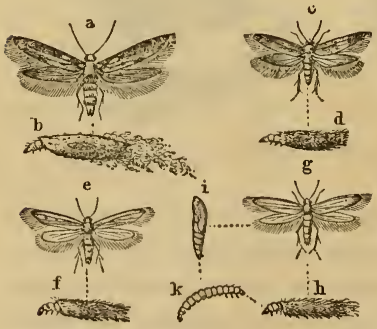

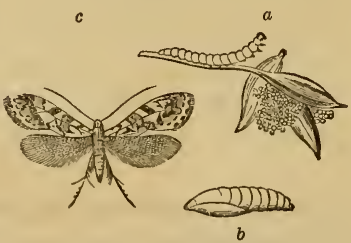

Fig. 85. Tinea granella. $a$ larva $-b$ crisalide $-c$ insetto perfetto.

Fig. 84. $a-b$ Tinea lacleella $-c-d$ Tinea spretella - e-f Tinea pelionella $g-i-h-k$ Tinea crinella.

Histoire des Insectes nuisibles à la vigne et particulièrement de la pyrale, etc., Paris, 1840-42.

Debbono essere menzionate fra i Piralidi le specie del genere Botys provviste di lunga proboscide (B. urticalis L., di color bianchiccio con macchie nere; è comune sulle ortiche); le specie del genere Asopia, che vivono alle spese di molte 
sostanze, come miglio, grano turco, grasso, miele, ecc., e sopratutto quelle del genere Nymphula, Hydrocampa, ecc., che passano lo stadio larvale. immerse nell'acqua. Non si conosce ancora intieramente il meccanismo della loro circolazione. In qualche caso le larve portano dei filamenti branchiali simili a quelli delle larve di molti Nevrotteri.

\section{Geometrini.}

Le due famiglie dei Pitometridi e dei Dendrometridi, che

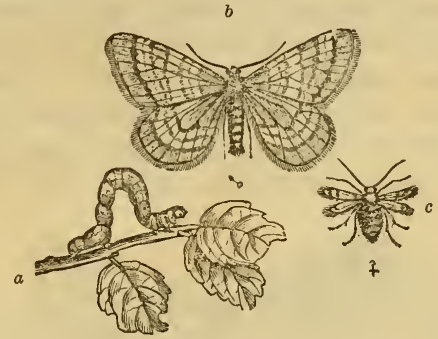

Fig. 86. Acidalia brumala $-a$ bruco $-b$ maschio $-c$ femmina.

formano il gruppo dei Geometrini, comprendono farfalle dalle ali grandi e larghe, dal corpo allungato, dai palpi mascellari non sviluppati e mancanti di ocelli. Caratteristico delle specie

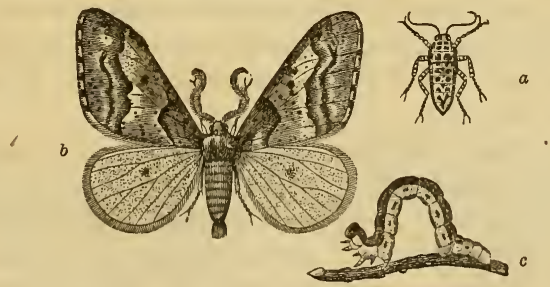

Fig. 87. Hibernia defoliaria $-a$ femmina $-b$ maschio $-c$ bruco.

di queste famiglie di Lepidutteri è il modo di camminare delle larve. Quando le larve vogliono procedere innanzi si fissano colle zampe anteriori, poi rialzando il loro corpo a mo' di arco, portano le zampe posteriori vicino alle anteriori, quindi si fissano colle zampe posteriori, protendono in avanti il corpo e colle zampe anteriori vanno a fissarsi nuovamente in un 
altro punto. Molte specie riescono spesso dannosissime alle piante da frutta. Sono dannosissime fra le altre l'Acidalia brumata, l'Hibernia defoliaria (notovole è la differenza che corre fra i due sessi in queste due specie; la femmina è attera ed ha le ali rudimentali) e talvolta anche la Zerene grossularia.

\section{NotтUini.}

Si distinguono i Nottuini facilmente dagli altri Lepidotteri per le antenne allungate e filiformi, per la proboscide mediocremente sviluppata, per i loro palpi relativamente grandi e pel loro corpo grosso, che va facendosi più o meno sottile nella sua parte posteriore. Le specie di questo gruppo, salvo poche eccezioni (Catocala, ecc.) hanno in generale piccole dimensioni; i loro colori sono talvolta molto vivaci ed eleganti. Le

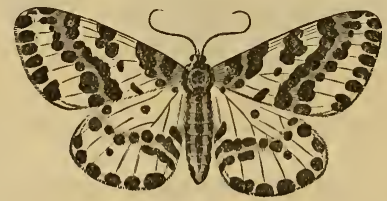

Fig. 88. Zerene grossularia.

larve, che sono per lo più prive di peli, compiono la loro metamorfosi o fra le fessure della scorza degli alberi o nella terra ai piedi degli alberi stessi. Moltissime sono le specie dei Nottuini conosciute e sono sparse per tutta la terra. Parecchie specie sono dannose a varie piante coltivate.

Il genere Plusia $\mathrm{Tr}$. è notevole per avere le ali anteriori sprovviste di squame; $P l$. jota, Pl. crysitis, ecc.

Degno di menzione è il genere Catocala Schr. che comprende specie relativamente grandi e colle ali inferiori elegantemente colorite in rosso vivissimo (C. clocata Esper. non rara fra noi), o in azzurro (C. fraxini Linn.), o anche in giallo, come si osserva in varie specie dell'America settentrionale.

Nel genere Agrotis le antenne sono filiformi nelle femmine, pettinate invece nei maschi; la proboscide è di mediocre lunghezza; le ali superiori non hanno colori vivaci; le inferiori sono di tinte più chiare e spesso hanno riflessi iridati. $\mathrm{Pa}-$ 
recchie specie di questo genere riescono spesso nocevoli ai cereali. La specie più nota è la $A$. segetum dalle ali anteriori bruno-gialliccie con piccole macchiette nere e dalle ali posteriori bianchiccie.

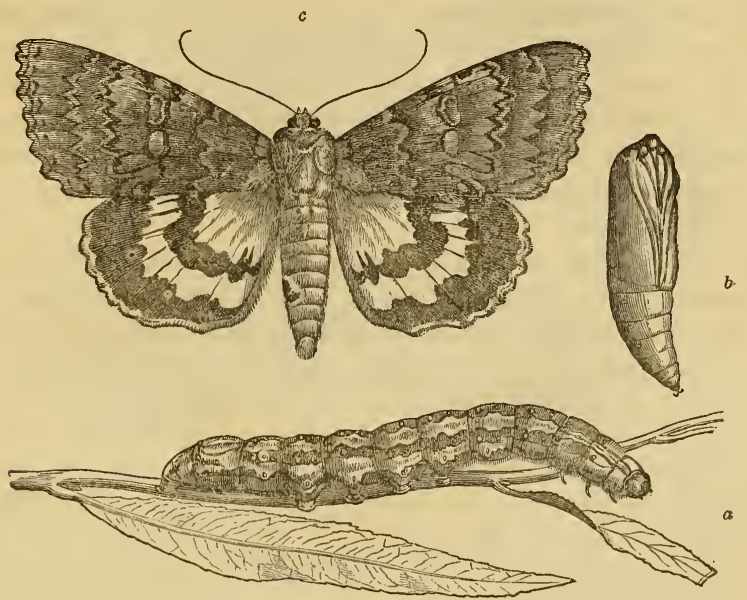

Fig. 89. Calocala elocala $-a$ bruco $-b$ crisalide $-c$ insetto perfetto.

Dannosissime, soprattutto a varie sorta di piante coltivate negli orti, sono le specie del genere Mamestra Tr. La specie più comune è la M. brassicae Linn.

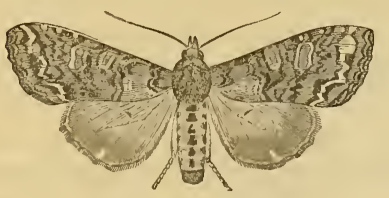

Fig. 90. Mamestra brassicae.

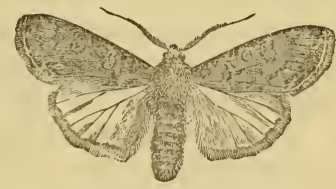

Fig. 91. Agrotis segetum.

\section{BoMbicini.}

Numerosissime sono le specie di Lepidotteri che costituiscono il gruppo dei Bombicini; gruppo che mentre comprende la specie più utile di tutti gli insetti, ha pure specie che spesso arrecano danni gravissimi all'uomo. 
I Bombicini sono caratterizzati dall'avere il corpo molto grosso, tozzo e ricoperto da peli più o meno fitti e più o meno lunghi. Le antenne sono allungate nella femmina, pettinate nei maschi. Vi hanno per lo più differenze ben spicccate fra i sessi: talvolta le femmine hanno ali rudimentali (Orgyia), o ne sono al tutto prive (Psyche, Heteroyynis, ecc.). I maschi invece sono buoni volatori. Le larve si filano un bozzolo più o meno grosso e resistente, nel quale passano lo stadio di crisalide. In qualche caso le larve fanno vita sociale. Le uova vengono deposte a mucchi e ricoperte da una sostanza spugnosa o lanosa.

I Bombicini fanno vita essenzialmente notturna; qualche specie tuttavia (Bombix salicis, B. dispar, ecc.) rola e si accoppia anche di giorno. In qualche specie si sono osservati casi di partenogenesi. I Bombicini sono sparsi per tutta la terra e presentano forme che sono fra le più grandi di tutti i Lepidotteri. I colori delle loro ali, soprattutto nelle specie esotiche, contrariamente a quanto si potrebbe credere, sono talrolta assai vivaci ed eleganti. I Bombicini ci offrono pure molti casi di mimismo sia nei colori, sia nella forma del corpo.

Il genere Lithosia comprende specie di piccola mole, a colori non molto vivaci ed i di cui bruchi variamente coloriti portano lungo il corpo dei tubercoletti rivestiti da ciuffi di peli. La specie più comune è la L. quadra Linn.

Molto eleganti pei colori delle ali sono le specie dei generi Euprepia Ochsh. e Callimorpha Latr. Non è rara fra noi la Euprepia caja, il di cui bruco è coperto di folti e lunghi peli. Non è specie nocevole. La maggior parte delle specie del genere Callimorpha sono esotiche. E specie europea non molto rara la $C$. dominola Linn.

Il genere Liparis è caratterizzato dall'avere le antenne corte e la proboscide poco sviluppata, od anche al tutto atrofizzata. Questo genere comprende le specie più dannose di tutti i Bombicini. Le specie più note sono le seguenti: L. salicis, in cui i due sessi sono dí color bianco, colle zampe anellate di nero. Questa specie è comunissima fra noi e vive per lo più sul pioppo. Talvolta essa si sviluppa siffattamente da distruggere completamente le foglie degli alberi su cui vive. Questa specie ci presenta un fatto singolare di policroismo nelle larve, delle 
quali parte sono macchiate di un bel colore giallo citrino e parte invece di bianco candido (1). Altre specie affini alla pre-

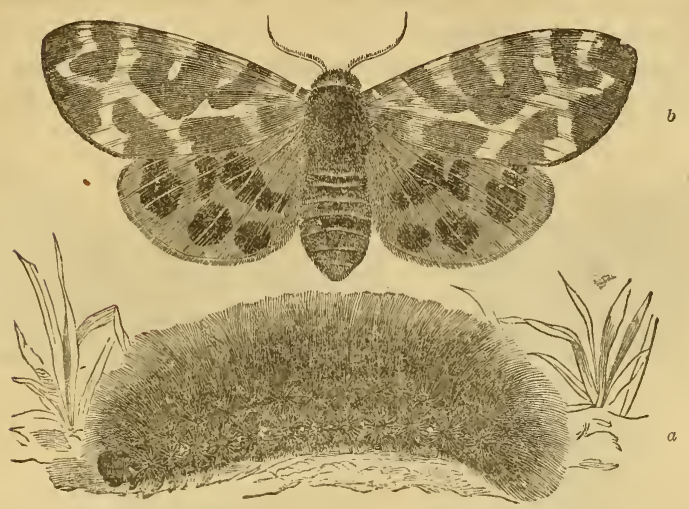

Fis. 92. Euprepia caja $-a$ bruco $-b$ insetto perfetto.

cedente sono pure non rare fra noi; fra le altre si possono menzionare la L. chrysorrhaea Linn. intieramente bianca,

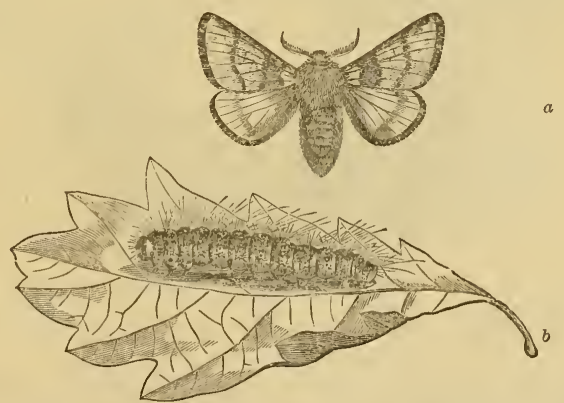

Fig. 93. Cnethocampa processionea $-a$ insetto perfetto $-b$ bruco.

meno gli ultimi segmenti dell'addome che sono di color bruno

(1) Si consulti a questo proposito L. Camerano: « Del Bombice del Salice nel contorno di Torino». Annali della $R$. Accademia d'Agricoltura di Torino, 1877. 
scuro e l'ano che è circondato da lanuggine di color giallo ferruginoso, e la L. auriflua Fabr. simile alla precedente, ma. un po' più piccola e colla estremità dell'addome coperta di peli di un bel color giallo.

Specie dannose assai sono pure la $L$. dispar, che vive sopra varie specie di piante, e la L. monacha, che vive su varie

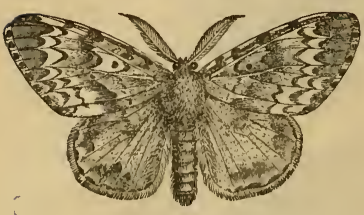

$a$

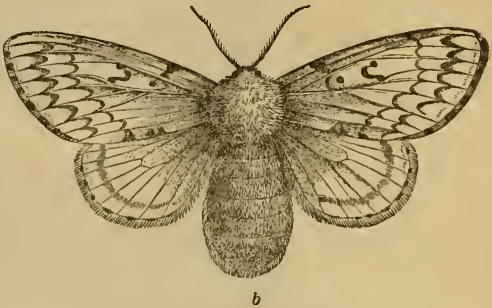

b

Fig. 94. Liparis dispar $-a$ maschio $-b$ femmina.

sorta di conifere. Affine al precedente è il genere Orgyia Ochsh. di cui (O. antiqua Linn.) le femmine sono attere.

Fra i Notodontidi troviamo specie multo dannose sotto due rispetti: dannose cioè alle piante di quercia sopra cui sogliono vivere e dannose per i peli delle loro lárve che, come ho già avuto occasione di avvertire, producono infiammazioni dolo-

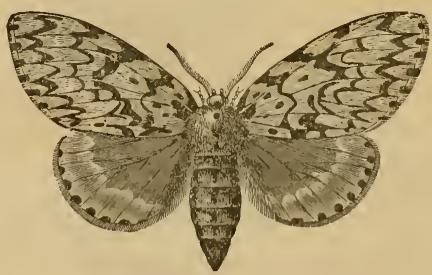

Fig. 95. Liparis monacha.

rose sulla pelle dell'uomo. La specie più comune è la Cnethocampa processionea Linn.

Importantissime, come è noto a tutti, sono le specie della famiglia dei Bombicidi. Basteri che io ricordi il comune filugello, B. mori e le specie affini: B. cynthia, B. yamamai, $B$. pernyi, ecc., le quali, secondo gli sperimenti fatti in questi ultimi tempi, possono tutte fornire delle qualità eccellenti di seta. 
Il genere Gastropacha Ochsh. comprende molte specie, alcune delle quali presentano fatti ben spiccati di mimismo $(G$. quercifolia Linn. dalle ali frastagliate che hanno, quando l'animale è in riposo, in tutto l'aspetto di foglie secche di quercia). Altre specie poi di questo genere riescono dannosissime a varie sorta di alberi di alto fusto. Ricorderò fra le altre la G. neustria Linn. e la G. pini Linn.

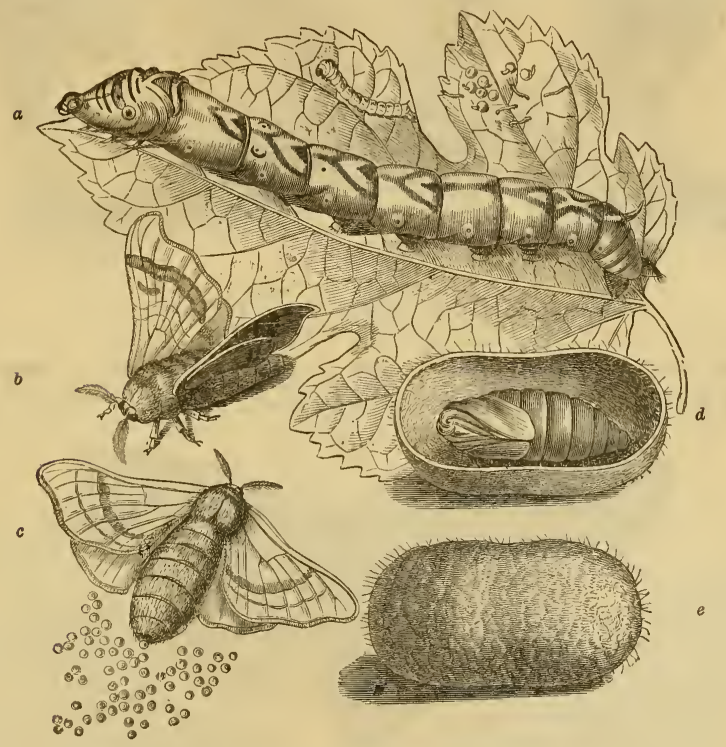

Fig. 96. Bombyx meri.

$a$ bruchi e uova in vari stadi di sviluppo $-b$ maschio $-c$ femmina che depone le uova $-d$ bozzolo tagliato con entro la crisalide $-e$ bozzolo intiero.

Il genere Saturnia Schr. comprende i più grossi fra tutti i Lepidotteri. Le sue specie sono spesso molto belle per la forma, pei colori e per la macchiettatura delle ali. Qualche specie può venir utilizzata per la produzione della seta. Fra noi sono specie comuni, la $S$. pyri Borkh., specie molto bella e grande che si sviluppa in primavera, e la $S$. carpini.

Molto importanti pei costumi sono gli Psichidi, Lepidotteri 
in generale, di piccola mole e di colori bruno-neri. Le femmine sono vermiformi e prive di ali. La femmina in qualche
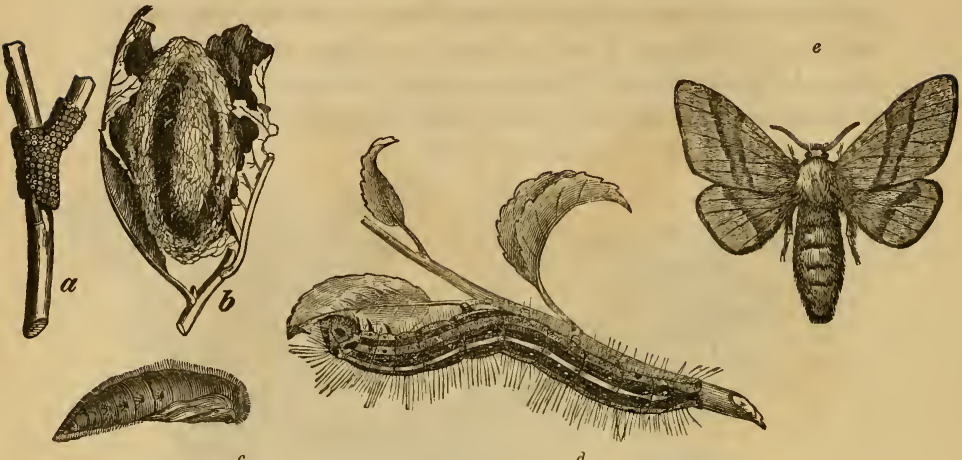

Fig. 97. Gastropacha neustria.

$a$ uova $-b$ bozzolo $-c$ crisalide $-d$ bruco $-e$ insetto perfetto.

caso non si muove affatto. Essa rimane in una sorte di sacco ed in questo, dopo che è stata fecondata, depone le uova. Tal-

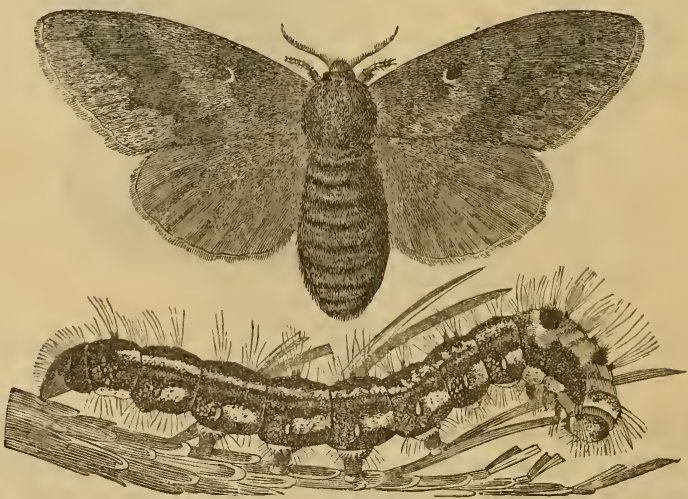

Fig. 98. Gastropacha pini.

volta il sacco è avvolto a spira (Cochlophanes helix Sieb.) e ricorda la forma degli astucci delle Helicopsiche fra i Neurotteri. 
I Zigenidi sono caratterizzati dalle antenne rigonfiate al loro apice, dal corpo allungato e grosso, dalle ali anteriori, in generale, molto più grandi delle posteriori e dai colori vivaci ed eleganti delle ali stesse. Le specie di questo genere abbondano fra

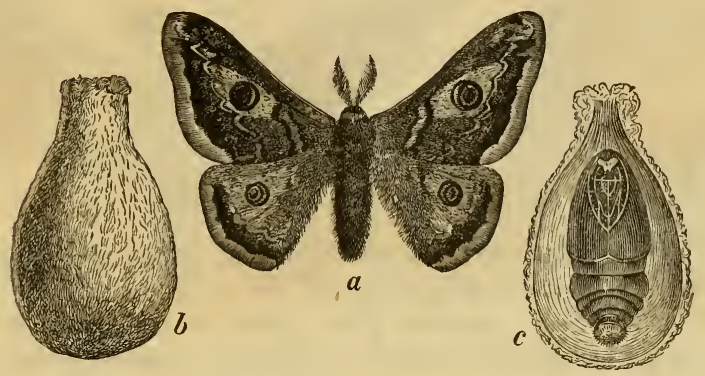

Fig. 99. Saturnia carpini.

$a$ maschio $-b$ bozzolo $-c$ bozzolo tagliato colla crisalide

noi soprattutto nelle praterie Alpine e fanno vita crepuscolare. La specie più comune è la $Z$. filipendulae Linn. Comune pure è la Syntomis phegea affine alla precedente.

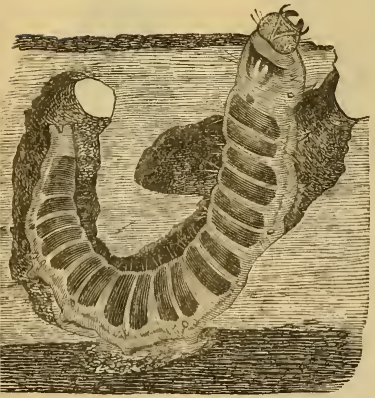

$a$

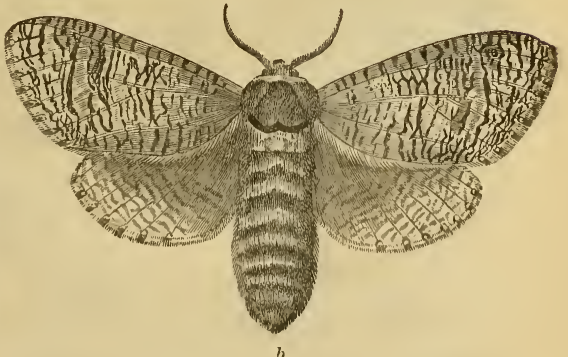

b

Fig. 100. Cossus lignipsrda $-a$ bruco $-b$ insetto perfetto.

La mancanza di proboscide caratterizza il genere Cossus, le di cui larve vivono nella midolla delle piante arrecando spesso danni gravissimi alle foreste. La specie più nota e più dannosa è il C. ligniperda Fabr. 


\section{SFINGINi.}

Nei lepidotteri di questo gruppo il corpo è grosso, allungato, e terminato posteriormente a punta; la proboscide è sempre ben sviluppata e talvolta lunghissima; le ali sono strette e per

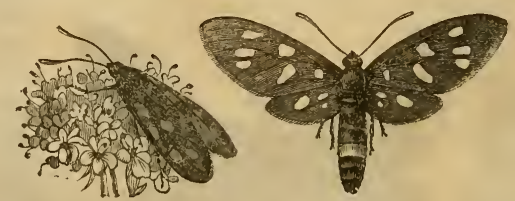

Fig. 101. Zygaena flipendulae, Fig. 102. Syntomis phegea.

lo più molto allungate; volano rapidissimamente; sono crepuscolari e si nutrono del nettare dei fiori.

Il genere Sesia presenta le ali spoglie in gran parte delle squamette caratteristiche dei Lepidotteri e comprende specie

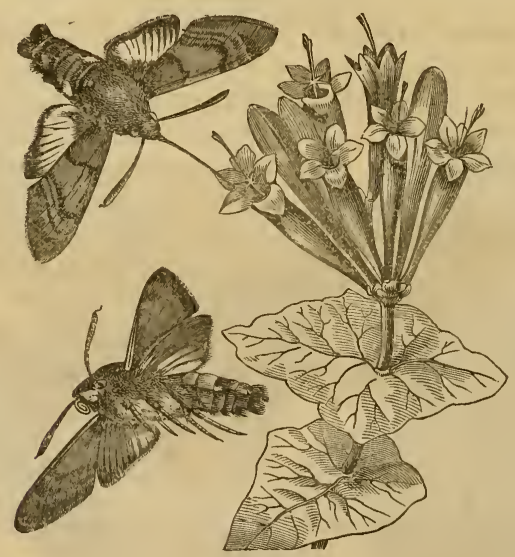

Fig. 103. Macroglossa stellatarum.

che ricordano le Api; le larve vivono nell'interno delle piante, non è rara la S. apiformis (Fig. 18).

Il genere Macroglossa, caratterizzato da una lunga proboscide, ha le antenne ingrossate verso l'apice da un ciuffo di peli, e l'addome rivestito pure alla sua estremità inferiore da un ciuffo di peli. Comune fra noi è la $M$. stellatarum Linn. 
Il genere Sphinx Linn. si distingue dal genere precedente principalmente per non avere il ciuffo di peli alla estremitá dell'addome. Numerosissime sono le specie di questo genere $\theta$

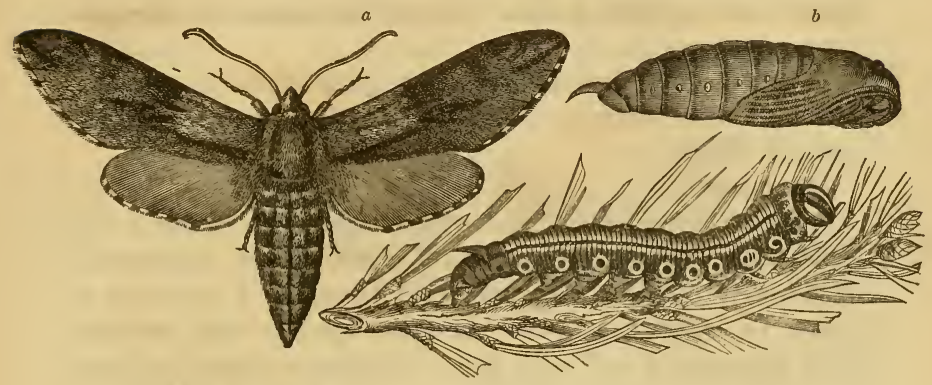

$c$

Fig. 104. Sphinic pinastri $-a$ insetto perfetto $-b$ crisalide $-c$ bruco.

spesso presentano le ali elegantemente colorite. Qualche specie arreca danni non lievi a varie sorta di piante; è in questo caso la $S$. pinastri che vive sulle conifere.
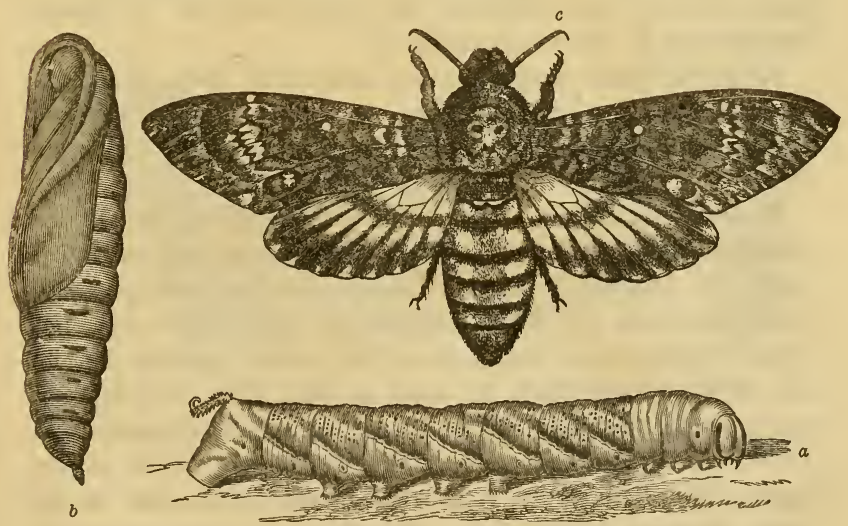

Fig. 105. Acherontia atropos $-a$ bruco $-b$ crisalide $-c$ insetto perfetto.

Il genere Acherontia è rappresentato fra noi da una specie di mole relativamente grande che vive nei campi di patate e che per la macchiettatura del suo torace piglia il nome di 
farfalla testa di morto. Questo Lepidottero è ghiottissimo deI miele e cerca di penetrare negli alveari. Le Api tuttavia sanno per lo più difendersi da questo nemico rimpicciolendo, col costrurre una muraglia di cera, l'entrata dell'alveare. A. atropos Linn.

Il genere Smerinthus Linn. non porta ciuffi di peli alla estremità delle antenne. Varie specie vivono fra noi. S. populi, S. tiliae, ecc.

ROPALOCERI.

I Ropaloceri, o lepidotteri diurni, comprendono le forme più belle e più eleganti, per vivacità di colori, di tutti i lepidotteri. Mancano di ocelli; le antenne sono allungate e terminate a clava. Le larve sono per lo più nude, qualche volta tuttavia sono ricoperte di peli e di spine; raramente tessono un bozzolo. I Ropaloceri sono sparsi per tutta la terra e abbondano principalmente nelle regioni equatoriali, dove hanno mole molto grande e dove rivestono colori splendidissimi. Sono buoni volatori e qualche specie è cosmopolita. I sessi si differenziano spesso fra loro nel colore e nella forma delle ali. Non sono rari i casi di ermafroditismo nel colore delle ali. In riposo tengono per lo più le ali verticali.

Hesperia Boisd. Questo genere comprende specie di piccola mole, a colori non molto spiccati, che vivono allo stato di larva principalmente sopra varie sorta di graminacee. $H$. sylvanus Fabr.

Piccole farfalline dai colori vivaci e dai riflessi metallici e dalle antenne quasi lunghe quanto il corpo costituiscono la famiglia dei Licenidi. I sessi differiscono fra loro principalmente nel colore delle ali. Sono frequenti fra noi le specie del genere Polyommatus Linn., e quelle del genere Lycaena, Boisd., che si vedono svolazzare in buon numero nelle praterie e nei campi sia del piano sia dei monti. La $L$. alexis rappresentata nella Fig. 106 ha due generazioni, una in maggio ed una in luglio. Ricordiamo pure il genere Thecla Linn., che ha gli occhi ricoperti di peli e che comprende un buon numero di specie che presentano spesso le ali posteriori con un prolungamento più o meno ben spiccato. È specie comune la $T$. walbum Illiger, ecc.

La famiglia dei Satiridi comprende un gran numero di ge- 
neri e di specie di cui la massima parte è esotica. Le specie di questa famiglia si distinguono per avere lè zampe anteriori atrofizzate. I colori delle ali sono spesso oscuri con macchie 0 striscie variopinte.

Satyrus Latr. Le ali sono per lo più di color bruno con una larga striscia chiara presso il margine esterno, la parte

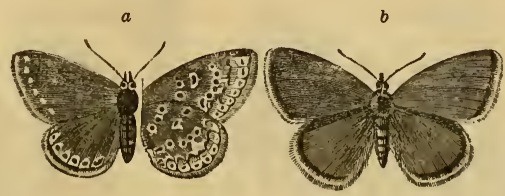

Fig. 106. Lycaena alexis $-a$ maschio $-b$ femmina.

inferiore delle ali posteriori è marmoreggiata. S.janira Och., S. briseis Linn., ecc., S. ida Esper.

Il genere Erebia Boisd. è affine al precedente, le specie tuttavia sono in generale più piccole ed hanno colori più cupi. Anche qui come nel genere Satyrus vi ha per lo più una striscia chiara presso il margine esterno delle ali. Le specie

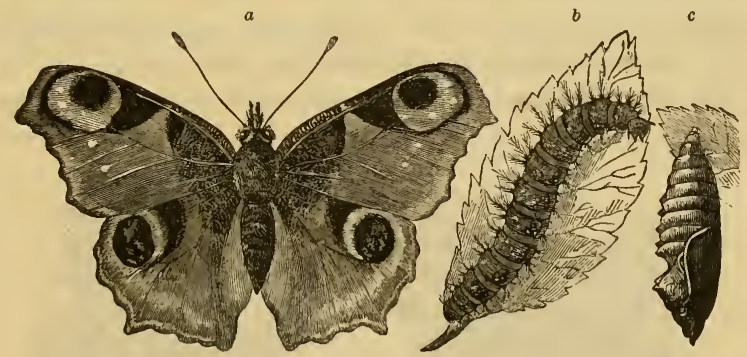

Fig. 107. Vanessa io $-a$ insetto perfetto $-b$ bruco $-c$ crisalide.

del genere Erebia abitano le regioni elevate dei monti. $E$. ligea Linn., ecc.

Le Vanesse, le Arginni e le Melitee dai colori eleganti e vivaci caratterizzano la famiglia dei Ninfalidi in cui le zampe anteriori sono atrofizzate e le larve sono coperte di spine e le crisalidi sono sospese per la loro estremità posteriore. Menzioneremo le specie seguenti comunissime fra noi : Vanessa io Linn.; ali superiormente rosso-brune con una macchia ocu- 
liforme azzurra chiara: Vanessa atalanta Linn.; ali superiormente nere veluttate, le anteriori hanno una striscia tra-
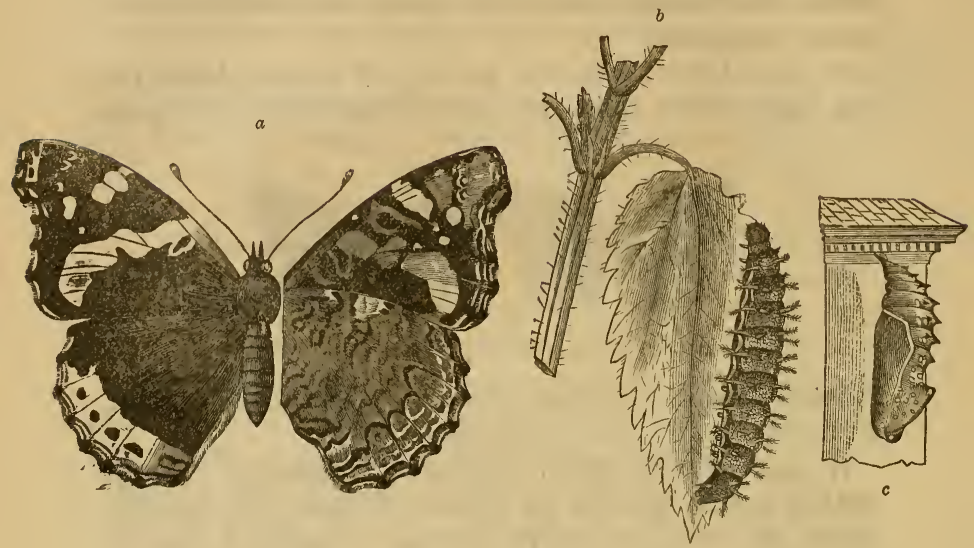

Fig. 108. Vaness $a$ atalanta $-a$ insetto perfetto $-b$ bruco $-c$ crisalide.

sversale di color rosso vivo, le posteriori, sono orlate esternamente di rosso-fuoco: Vanessa urticae Linn., ecc.

Argynnis paphia Linn. superiormente di color giallo bruno

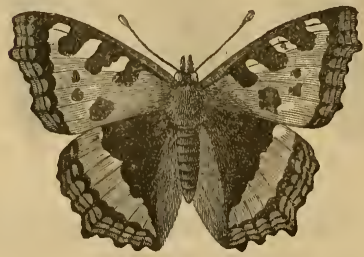

Fig. 109. Vanessa urticae.

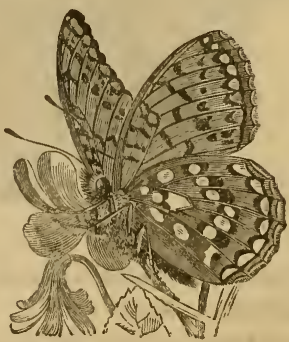

Fig. 110. Argynnis paphia.

con macchie nere, inferiormente giallo chiaro con macchie nere e lucenti e madreperlacee.

La famiglia delle Pieridi è costituita da lepidotteri di mole mediocre, dalle ali di colori chiari e appariscenti, bianco, 
giallo-citrino, rosso, ecc. Sono buoni volatori e sono sparsi per tutta la terra. Fra noi sono comuni assai le specie seguenti: Pieris brassicae Linn., o cavolaia maggiore, bianca con qualche macchia nera. P. crataegi Linn., pure bianca ma colle nervature delle ali nere. $P$. cardamines Linn., di cui il maschio

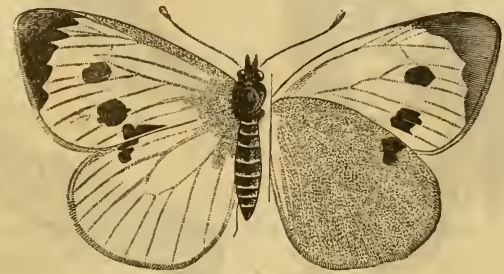

Fig. 111. Pieris brassicae.

ha la metà delle ali anteriori di color giallo vivace ed inferiormente macchiettate di verde.

Le famiglie dei Danaidi e dei Eliconidi comprendono specie elegantissime ma proprie quasi esclusivamente delle regioni equatoriali.

Gli Equitidi hanno antenne corte e rigonfiate alla estre-

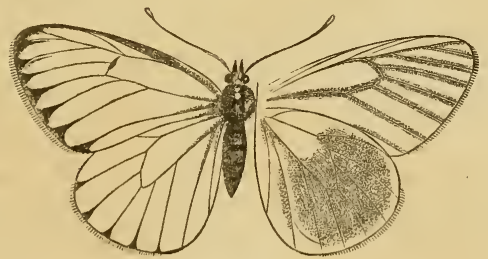

Fig. 112. Pieris crataegi.

mità, zampe anteriori ben sviluppate, ali posteriori prolungate generalmente in una coda più o meno spiccata e bruchi con una appendice forcuta sul collo. Non sono rari in questa famiglia i casi di polimorfismo sessuale.

Gli Equitidi comprendono gli elegantissimi Papilio Linn., rappresentati fra noi dalle specie seguenti : P. machaon Linn., $P$. podalirius Linn., ecc. 
Il genere Doritis ha fra noi una specie la D. apollo, Linn., che vive sulle alte regioni alpine.

Il genere Ornithoptera, finalmente, comprende i più grossi

$a$

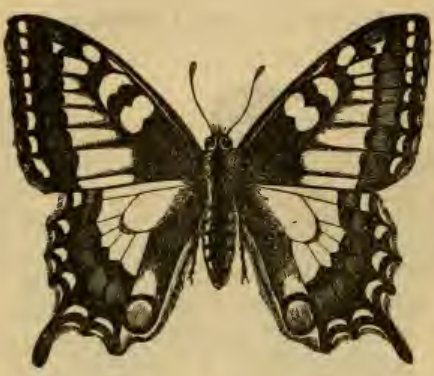

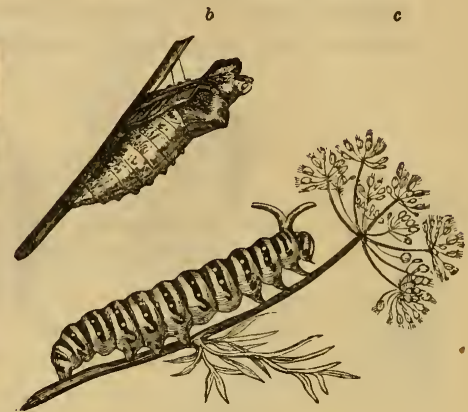

Fig. 113. Papilio machaon $-a$ insetto perfetto $-b$ crisalide $-c$ bruco.

fra tutti i lepidotteri diurni ed è proprio della regione IndoMalese. Le specie di questo genere sono vestiti di colori vivacissimi e hanno un volo molto alto e rapidissimo. O. priamus (Linn.). 


\section{Bibliografia.}

Il numero degli scritti riguardanti la classificazione e la descrizione dei Lepidotteri è grandissimo, io non ricorderò che i lavori più importanti e generali: ricorrendo a questi si potranno avere maggiori indicazioni bibliografiche.

P. Cramer. - Papillons exotiques des trois parties du monde, l'Asie, l'Afrique et l'Amerique, ressemblés et décrits, etc. (Testo olandese e francese), 1775.

E. J. Esper. - Die Europäischen Schmetterlinge in Abbildungen nach der Natur., ecc., 1777-1805.

F. Ochsenheimer. - Die Schmetterlinge von Europa. Leipzig, 1807-1816.

J. HüBner. - Sammlung europäicher Schmetterlinge. Amburg, 1805-1824. L'opera dell'Hübner venne continuata da G. Geyer, e più tardi da HerrichSchäffer.

Herrich-SCHAEFFER. - Systematische Beschreibung der Schmetterlinge von Europa. Regensburg, 1843-1855. Lepidopt. exaticor. species novae aut minus cognitae, 1850-1865.

Godart et Duponchel. - Histoire naturelle des Lépidoptères de France, vol.XI, Parigi, 1821-1840.

- Iconographie des Chenilles, Paris, 1832-1849.

Staudinger e Wocke. - Catalog der Europäischen Schmetterlinge. Dresden, 1871.

E. Berce. - Faune entomologique Française. Lépidoptères. Paris, 1870-73.

Borsduval. - Species général des Lépidoptères. Paris, 1836-74.

P. Rambur. - Catalogue systématique des Lépidoptères de l'Andalusie. Paris, 1858-66.

C. e R. Felder. - Novara expedition Lepidoptera. Wien, 1865-74.

V. Ghulani. - Elenco delle specie di Lepidotteri riconosciute esistenti negli Stati Sardi. Mem. della R. Accad. delle Sc. di Torino, serie II, vol. XIV, 1852.

A. Cunò. - Saggio di un catalogo dei Lepidotteri d'Italia. Bull. della Soc. Ent. Italiana, vol. VI e seguenti.

Buchanan White. - Armure génitale des Lygaenidae. Ann. de la Soc. Ent. de France, serie 5 ${ }^{\mathrm{a}}$, vol. VIII, 1879. 


\section{XX. \\ G O L E OTTER I}

Insetti con apparato boccale foggiato per masticare; ali anteriori trasformate in elitre; metamorfosi compinte.

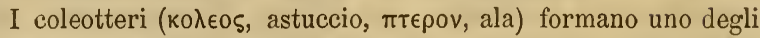
ordini più naturali degli insetti e nello stesso tempo anche uno dei più numerosi.

Le forme dei coleotteri, quantunque non si scostino mai da un unico piano generale di struttura, variano grandemente, e, come si può scorgere da quanto si è detto nei primi capitoli di questo libro, si trovano in esse numerosi esempi di forme mimetiche e di strutture apparentemente anormali che sono il portato della scelta naturale e della scelta sessuale.

Il capo nei coleotteri è sempre ben sviluppato e in qualche caso (vedi cap. IV e V) porta dei prolungamenti a mo' di corna più o meno lunghi e più o meno complicati. Generalmente il capo è incassato nel torace; talvolta invece presenta un collo ben spiccato. In qualche specie il capo è più grosso del protorace stesso (Myrmecoptera egregia Germ.). In molti casi il capo è prolungato anteriormente a mo' di becco (Curculionidi). Le antenne variano moltissimo nella forma, ora sono allungate e filiformi, ora terminate a clava, ora coperte di un numero vario di lamelle, ora pettinate, ora dentate, ora diritte, ora piegate ad angolo; talvolta sono nude, talvolta invece fittamente pelose.

Gli occhi per lo più sono sviluppatissimi, in qualche caso tuttavia sono intieramente atrofizzati (vedi pag. 94 e 117).

L' apparato boccale è foggiato per masticare ed è in generale molto sviluppato.

Le mandibole, sviluppandosi enormemente nei maschi di certe specie, forniscono importanti caratteri sessuali secondari.

Il torace ha il primo segmento molto sviluppato. La forma del torace è variabilissima, e sarebbe cosa troppo lunga il menzionare tutte le modificazioni che essa presenta. Anche il torace presentasi frequentemente diverso nei due sessi. 
Le ali nei coleotteri sono in numero di quattro; le due anteriori dure e trasformate in elitre, le due posteriori membranose e ripiegate sotto alle prime. Tutte le ali possono mancare intieramente nelle femmine di varie specie, oppure possono essere rudimentali. In molti casi mancano le ali membranose (molti carabici) e non vi hanno che le elitre; in altri casi non solo mancano le ali membranose, ma le elitre si saldano fra loro in modo che rendono all'insetto affąto impossibile il volare (Cychrus). Lo sviluppo poi delle ali e delle elitre è vario. Ora le elitre ricoprono l'addome, ora sono più lunghe dell'addome stesso, ora ne ricoprono solo i primi articoli (brachelitri).

L'addome è sempre relativamente grande, non peduncolato, e di forma poco variabile. Talvolta parecchi segmenti si saldano fra loro.

Le zampe sono in generale ben sviluppate e sono variamente modificate. Ora le anteriori sono allungate e foggiate ad organi di presa; ora le mediane e le posteriori sono appiattite, rivestite di peli-laminette e foggiate a remi ; ora le posteriori sono molto ingrossate e foggiate al salto. Il numero degli articoli dei tarsi è variabile e fornisce buoni caratteri per la distinzione dei vari gruppi di coleotteri.

I coleotteri sono sparsi per tutta la terra e abbondano nelle regioni equatoriali, ma non sono rari anche nelle regioni più fredde e settentrionali; vivono pure a grandi altezze sui monti. Una parte-dei coleotteri fa vita acquatica, ma non ha respirazione branchiale. Qualche specie vive nelle acque del mare presso alle coste. I coleotteri presentano molto spiccati i caratteri sessuali secondari e i fatti di mimismo (cap. III e IV).

I coleotteri hanno metamorfosi compiute. Le larve hanno, come gli insetti perfetti, l' apparato boccale foggiato per masticare; ora esse sono apode, ora invece hanno zampe più o meno ben sviluppate. Si osservano non raramente traccie di zampe sugli ultimi segmenti addominali. Le larve hanno ocelli. Qualche specie presenta il fatto della ipermetamorfosi di cui abbiamo già detto nel capitolo XIII.

I coleotteri, dirò in ultimo, sono ad un tempo utili e dannosi all' uomo. Riescono utili, ad esempio, i coleotteri carnivori, i quali distruggono una grande quantità di larve di altri insetti nocevoli, ma sono invece spesso dannosissimi i coleotteri fitofagi, i quali guastano molte delle piante dall'uomo coltivate. 
Tavola di classificazione dei Coleotteri.

Criptotetrameri SCocinellidi, Coccinella, Chilocorus. - En(Pseudotrimeri) domichidi, Lycoperdina.

Crisomelidi, Cassida, Hispa, Galeruca, Haltica, Chrysomela, Cryptocephalus, Timarea, Donacia. - Cerambicidi, Leptura, Saperda,

Criptopentameri (Pseudotetrameri)

Eteromeri . . . Clytus, Callidium, Aromia, Cerambyx, Prionus. - Bostricidi, Bostrychus, Hylesinus. - Curculionidi, Calandra, Balaninus, Otiorhynchus, Apion, Rhynchites, Entimus. - Bruchidi, Bruchus.

Edemeridi, Oedemera. - Meloidi, Meloë, Lytta, Sitaris. - Ripiforidi, Rhipiphorus. - Mordellidi, Mordella. - Pirocroidi, Pyrochroa, Anticus. - Melandridi, Melandrya. - Cistelidi, Cistela. - Tenebrionidi, Tenebrio. - Elopidi, Helops. - Pimelidi, Opatrum, Blaps, Pimelia.

Silofagidi, Cis, Anobium, Ptinus. - Cleridi, Clerus, Trichodes. - Malacodermidi, Drilus, Cantharis, Lampyris. - Atopidi, $D a$ scillus. - Elateridi, Lacon, Elater. - Buprestidi, Buprestis, Dicerca. - Lamellicornidi, Lucanus, Ateuchus, Copris, Onthophagus, Aphodius, Melolontha, Dynastes, Cetonia. - Birridi, Nosodendron, Byrrhus. - Troxidi, Trox. Dermestidi, Dermestes, Attagenus, Anthrenus. - Criptofagidi, Mycetophagus. Cucujidi, Cucujus. - Colididi, Colydium. - Nitidulidi, Nitidula. - Isteridi, Hister. Pentameri ... - Tricopterigidi, Trichopteryx. - Sferidi, Sphaerius. - Silfidi, Silpha, Necrophorus. Anisotomidi, Anisotoma. - Scidmenidi, Scydmenus. - Pselafidi, Pselaphus. Clavigeridi, Claviger. - Pausidi, Paussus. - Stafilinidi, Aleochara, Myrmedonia, Staphylinus, Stenus. - Omalinidi, Omalium. - Idrofilidi, Hydrophilus, Hydrous, Cercyon. - Ditiscidi, Hydroporus, Agabus, Dytiscus, Cybister, Acilius. - Girinidi, Gyrinus, Orectochilus, Gyretes. - Carabidi, Bembidium, Trechus, Harpalus, Feronia, Pterosticus, Anchomenus, Chlaenius, Clivina, Brachinus, Scarites, Calosoma, Nebria, Cychris, Carabus, Omophron, Mormolyce, Cicindela, Megacephala. 
$A$. Tarsi di quattro articoli, di cui uno è rudimentale (Criptotetrameri).

a. Torace senza solcature: corpo convesso, quasi emisferico.

$a^{\prime}$. Torace con tre solcature alla base: capo allungato.

Coccinellidi.

Endomichidi.

$A^{\prime}$. Tarsi di cinque articoli di cui uno è rudimentale e nascosto (Criptopentameri).

a. Corpo corto, arrotondato, convesso: antenne allungate, filiformi : addome con cinque lamine ventrali. Crisomelidi.

$a^{\prime}$. Corpo allungato: antenne relativamente molto lunghe: più lunghe nei maschi che non nelle femmine. Cerambicidi.

$a^{\prime \prime}$. Corpo di piccola mole e cilindrico: capo nascosto nel protorace: antenne corte e pettinate, alquanto ingrossate al loro apice . . . . . . Bostricidi.

$a^{\prime \prime \prime}$. Corpo ora allungato e cilindrico: ora tozzo e quasi sferico: capo prolungato anteriormente a mo' di proboscide: antenne per lo più piegate ad angolo. . . Curculionidi. $a^{\text {Iv }}$. Corpo compresso e corto: occhi grandi e sporgenti : capo prolungato anteriormente a mo' di becco. Bruchidi.

$A^{\prime \prime}$. Tarsi delle zampe anteriori e mediane di cinque articoli: tarsi delle zampe posteriori di quattro articoli . Eteromeri.

a. Corpo stretto ed allungato: antenne filiformi : elitre che non ricoprono lateralmente tutto l'addome . Edemeridi.

$a^{\prime}$. Capo largo, ristretto posteriormente a mo' di collo: elitre larghe, divaricate e che non ricoprono tutto il corpo.

Meloidi.

$a^{\prime \prime}$. Antenne dentellate nelle femmine, pettinate nei maschi: elitre della lunghezza del corpo . . . Ripiforidi.

$a^{\prime \prime \prime}$. Corpo di piccola mole, allungato e cuneiforme: antenne filiformi . . . . . . Mordellidi.

$a^{\text {Iv }}$. Capo piegato e più largo che non l'orlo anteriore del protorace, e ristretto posteriormente a mo' di collo. Pirocroidi.

$a^{\vee}$. Capo triangolare e più o meno incassato nel protorace: antenne corte di dieci od undici articoli. Melandridi.

$a^{\mathrm{v}}$. Capo non ristretto posteriormente a mo' di collo: uncini delle zampe pettinati . . . . Cistelidi.

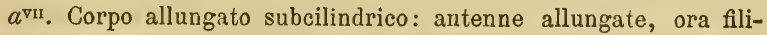
formi, ora ingrossate all'apice . . Tenebrionidi. $a^{\text {vir. }}$. Corpo convesso globoso: le ali membranose mancano quasi sempre: le elitre sono per lo più saldate insieme. Pimelidi. $A^{\prime \prime \prime}$. Tarsi di cinque articoli (Pentameri). 
a. Corpo lungo, per lo più cilindrico: antenne filiformi nella femmina, pettinate nel maschio ed inserite davanti agli occhi.

Silofagidi.

$a^{\prime}$. Corpo coperto da peli: colori vivaci : elitre cilindriche.

Cleridi.

$a^{\prime \prime}$. Corpo molle: antenne dentate o pettinate: talvolta i tarsi anteriori dei maschi hanno quattro articoli. Malacodermidi. $a^{\prime \prime \prime}$. Corpo allungato: gli angoli posteriori del protorace sono prolungati in punta. L'insetto quando è coricato sul dorso può pel modo speciale di articolazione del protorace e del mesotorace, mediante un salto ritornare nella posizione normale.

Elateridi.

$a^{\mathrm{Iv}}$. Corpo molto allungato e terminato posteriormente in punta: capo piccolo e molto incassato nel protorace: colori eleganti e metallici, antenne dentate o pettinate . Buprestidi.

$a^{v}$. Corpo grosso, tozzo più o meno cilindrico: antenne di sette od undici articoli, di cui il primo ingrossato e gli ultimi appiattiti e disposti come le bacchette di un ventaglio.

Lamellicornidi.

$a^{\mathrm{vr}}$. Corpo quasi sferico: le coscie presentano una solcatura nella quale vengono a collocarsi ripiegandosi le tibie. Birridi.

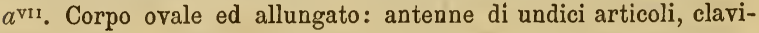
formi

Dermestidi. $a^{\text {vir. }}$ Corpo allungato: antenne claviformi: tarsi di tre o sei articoli

Criptofagidi. $a^{\mathrm{IX}}$. Corpo appiattito ed allungato: antenne per lo più filiformi.

Cucujidi.

$a^{\mathrm{x}}$. Corpo molto allungato: antenne ora alquanto ingrossate all'apice, ora fusiformi

Colididi. $a^{\mathrm{xI}}$. Corpo di piccola mole e arrotondato: antenne claviformi.

Nitidulidi.

$a^{\mathrm{xil}}$. Corpo allargato: ovale e mediocremente convesso. Il protorace è profondamente incavato anteriormente: le elitre sono corte e non ricoprono tutto l'addome: vivono nelle materie animali in decomposizione. . . . Isteridi. $a^{\mathrm{x} I 11}$. Corpo di piccola mole, ora liscio, ora ricoperto di peluzzi: antenne di undici articoli: gli ultimi sono alquanto ingrossati.

Tricopterigidi. $a^{\mathrm{xIv}}$. Corpo ora appiattito, ora più o meno cilindrico: antenne claviformi : zampe posteriori spesso robustissime e atte a scavare

Silfidi. $a^{\mathrm{xv}}$. Corpo di piccolissime dimensioni: antenne relativamente 
lunghe, claviformi : elitre corte e che non ricoprono che i primi segmenti addominali. . . . . Pselafidi. $a^{\mathrm{xvI}}$. Corpo molto allungato e più o meno appiattito: elitre molto corte: addome quasi intieramente ricoperto Stafilinidi. $a^{\mathrm{xvII}}$. Corpo ovale, convesso superiormente: più o meno carenato

inferiormente: antenne corte e claviformi : vivono nell'acqua in cui si muovono lentamente o nelle materie animali in decomposizione . . . . . Idrofilidi. $a^{\mathrm{x} \text { uI. }}$. Corpo ovale ed appiattito: antenne filiformi: zampe posteriori foggiate a remi: vivono nell'acqua ed hanno nuoto rapidissimo

Ditiscidi. $a^{\mathrm{xrx}}$. Corpo ovale, per lo più molto convesso: occhi divisi in due: nuotano descrivendo numerosi giri sulla superficie dell'acqua

Girinidi.

$a^{\mathrm{xx}}$. Corpo di forma varia: zampe e mandibole ben sviluppate e robuste: ali membranose spesso mancanti: elitre talvolta saldate assieme: antenne filiformi di undici articoli : sono carnivori e predatori

Carabidi.

I coccinellidi comprendono piccoli insetti dal corpo molto convesso e dai colori vivaci. Il loro studio è molto difficile. Sono comuni fra noi molte specie dei generi Coccinella, Adalia, Thea, ecc., le quali sono utilissime alla agricoltura distruggendo un gran numero di Aphis e di Trips. C. septempunctata Linn., di color rosso con macchie rotonde nere. T. vigintiduo-pun-

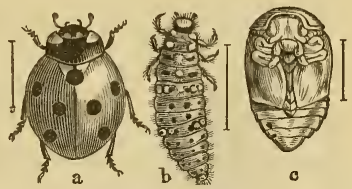

Fig. 114. Coccinella septempunctata. $a$ insetto perfetto - $b$ larva $c$ crisalide. ctata Linn., più piccola della precedente e di color giallo-chiaro con molti punticini neri.

Oltre a diecimila sono le specie che costituiscono la famiglia dei Crisomelidi. Gli insetti di questa famiglia sono fitofagi e spesso riescono dannosissimi alle piante coltivate dall' uomo. Basterà che io menzioni la Doriphora decemlineata, e l'Haltica oleracea.

Il genere Cassida ha il corpo appiattito a mo' di scudo e comprende specie ornate ora di eleganti colori madreperlacei, ora di colori simili a quelli delle piante sopra cui vivono. $C$. equestris Linn. C. margaritacea Schall.

Il genere Hispa è notevole pel corpo irto di spine. L' $H$. 
atra Linn., piccolo coleottero di color nero è specie comune fra noi.

Il genere Haltica, Ill., che comprende molte specie di pic-

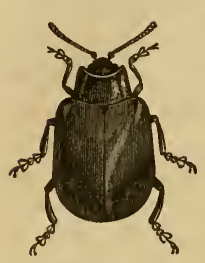

Fig. 115. Chrysomela populi. cola mole che vivono sopra un grande numero di piante, è notevole per le zampe posteriori ingrossate ed atte al salto (1). $H$. oleracea Fabr., ecc.

Le specie del genere Chrysomela hanno per lo più colori assai eleganti e splendidi riflessi metallici. C. menthastri Suff. Affini al genere Chrysomela sono pure i generi: nostre Alpi ornate di bellissimi colori, Lamprosoma Kirb., ecc. Menzionerò finalmente il genere Donacia

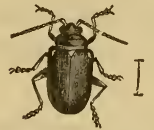

Fig. 116.
Haltica oleracea. che ha vita acquatica nel periodo larvale, e che per la forma del corpo fa il passaggio fra i Cerambicidi ed i Crisomelidi. Le specie di questo genere sono molte ed hanno colori metallici. D. crassipes Fabr.

Numerosissimi sono i generi e le specie di coleotteri com-
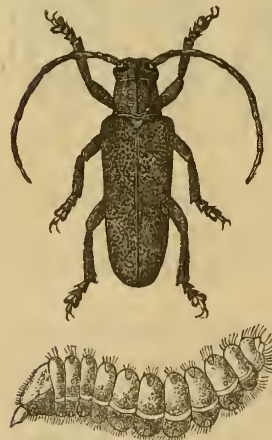

Fig. 117 Saperda carcharias colla sua larva. presi nella famiglia dei Cerambicidi, famiglia che corrisponde essenzialmente ai Longicorni di molti autori. La mole è variabilissima: vi hanno Cerambicidi di pochi millimetri di lunghezza, e altri di oltre un decimetro. I colori sono per lo più assai vivaci ed eleganti. Le specie di questa famiglia sono sparse per tutta la terra; vivono per lo piú allo stato di larva nei tronchi e nei rami degli alberi in cui essi scavano numerose gallerie. Molte specie sono, sviluppandosi in grande numero, dannose alle foreste. Il genere Leptura Linn. comprende specie di piccola mole, in generale di color

(1) Si consulti a questo proposito P. BargagLI a La flora delle Altiche in Europa ». Bull. della Soc. Ent. Ital., anno X, 1878. 
rosso con macchie nere più o meno spiccate, che vivono sui fiori, L. rubra Linn.

Il genere Saperda Fabr., e Lamia Fabr., hanno specie in generale di mole mediocre e di colori poco vivaci. S. carcharias Linn., di color bigio o rossiccio. L. textor Linn., di color nero cupo. Ricorderò ancora l'Acrocinus longimanus pel grande sviluppo delle zampe anteriori del maschio (vedi cap. IV e V).

Clytus Fabr., Callidium Fabr. Le specie di questi generi hanno mole mediocre, colori vivaci ed eleganti macchiettature e vivono sui fiori. Sono specie comuni fra noi il $\mathrm{Cl}$. arcuatus Linn., il Call. sanguineum Linn. ed il Call. violaceum Linn.

I generi Aromia, Cerambyx, Prionus, ecc., comprendono specie di mole relativamente

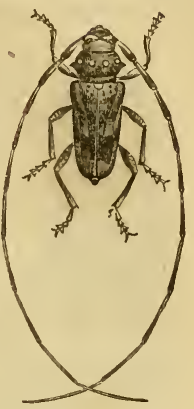

Fig. 118. Lamia aedilis. grande e ornate ora di colori bruni (Cerambyx), ora invece

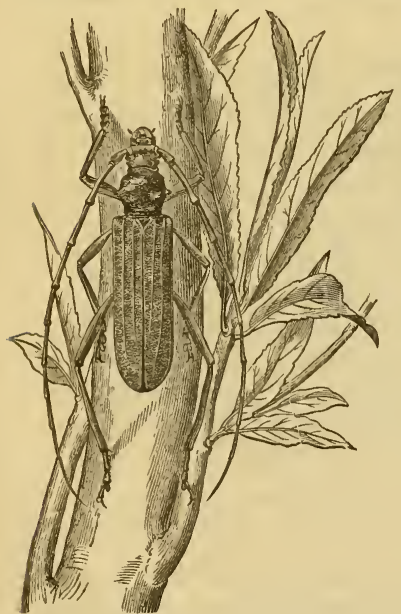

Fig. 119. Aromia moschata.

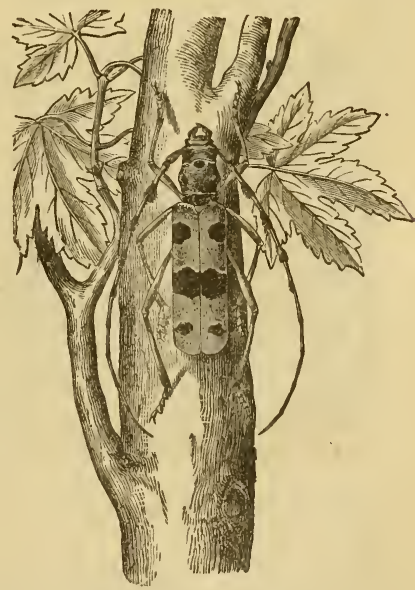

Fig. 120. Rosalia alpina.

di colori vivaci ed eleganti (Aromia, Callichroma, ecc.). Noti a tutti sono l'A. moschata Linn. pel suo gradevole odore, e 
il Cerambyx heros. Sulle Alpi vive la bellissima Rosalia alpina Linn.

I Bostricidi sono piccoli coleotteri dal corpo cilindrico che scavano allo stato di larva numerosissime gallerie nei tronchi

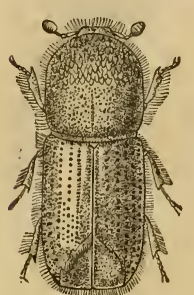

Fig. 121. Bostrychus typogra phus (ingrandito). degli alberi, soprattutto delle conifere. Sono dannosissimi: Hylesinus fraxini Fabr., e varie specie di Bostrychus. Il B. typographus Linn. è nocevolissimo alle foreste di pini.

Fra i Curculiondi ricorderò i generi seguenti :

Calandra, che comprende specie dannose a varie sorta di piante. C. granaria Linn., piccolo coleottero di color bruno che vive nel grano. Il genere Rhynchophorus Herbst è affine al precedente; la specie più nota di questo genere è il $R$. palmarum, di color nero vellutato, che vive nelle regioni equatoriali.

Il genere Balaninus Germ. comprende specie che vivono allo stato di larva in vari frutti. $B$. nucum.

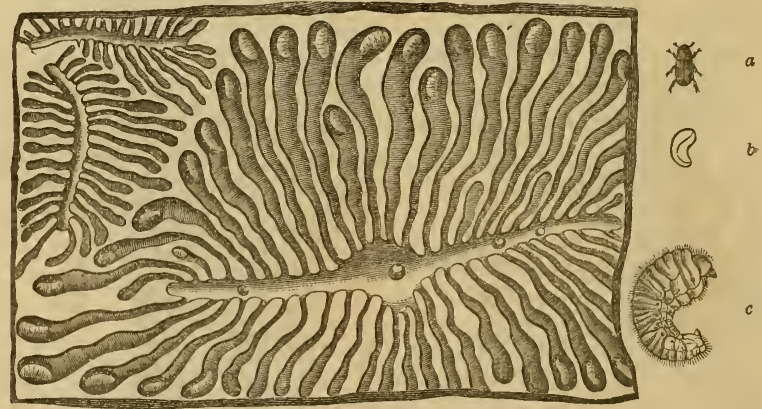

Fig. 122. Gallerie del Bostrychus typographus.

$a$ insetto perfetto di grandezza naturale $-b$ crisalide $-c$ larva (ingrandita).

Un grandissimo numero di specie dall' addome ora sferico ora più o meno appiattito e dall' integumento molto duro costituisce il genere Otiorhynchus. Qualche specie di questo genere, $O$. picipes Fabr., riesce dannosa in alcune località alle viti. 
Nocevolissime soprattutto alle foreste di conifere sono le specie del genere Hylobius di cui la larva rode il legno dei tronchi, e l'insetto perfetto principalmente le gemme. $H$. pini.

Sono degni di nota per gli elegantissimi riflessi metallici del

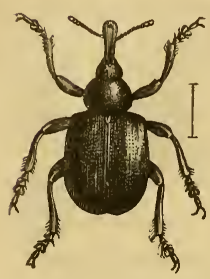

Fig. 123.

Hylobius pini.

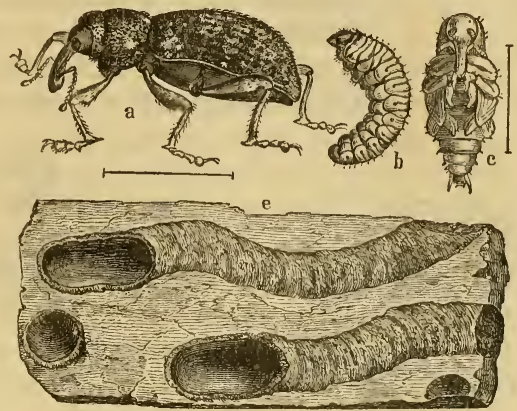

Fig. 124. Rhynchiles beluleti - $a$ insetto perfetto $b$ larva $-c$ crisalide $-e$ gallerie scavate nel legno.

loro corpo ricoperto di piccole squame simili a quelle delle ali dei Lepidotteri i generi Polydrosus, Cyphus, Platyomus, Entimus, ecc.

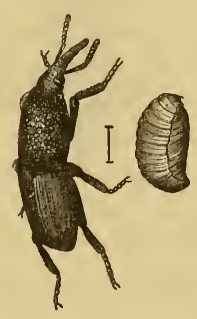

Fig. 125.

Calandra granaria in vari stadi di sviluppo.
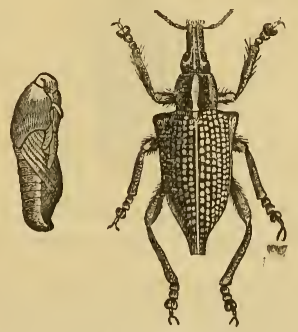

Fig. 126.

Entimus imperialis.

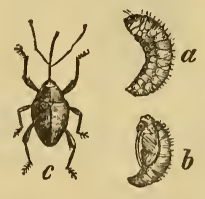

Fig. 127. Balaninus nucum - a larva $b$ ninfa $-c$ insetto perfetto.

Il genere Apion comprende un grandissimo numero di specie di piccolissima mole, dal corpo piriforme, che vivono sopra molte specie di vegetali. $A$. pisi, ecc.

Le specie del genere Rhynchites sono notevoli per i bei 
colori che esse, in generale, ci presentano, e per i danni che qualcuna di esse porta a varie sorta di piante. Il $R$. betulet $i$ Fabr. intacca i gambi della vite e depone le uova nelle foglie che accartoccia e fa arvizzire.

Il genere Brenthus Fabr. è caratterizzato dal corpo allungatissimo e molto stretto. Le specie che lo costituiscono abitano le regioni calde dell'Asia e dell'America.

Bruchus Linn. Questo genere è affine ai Carculionidi per alcuni caratteri e pei costumi. Il B. pisi Linn. è dannosissimo ai piselli entro $i$ quali vive allo stato di larva.

Il genere Oedemera Oliv. ha il corpo allungato, le antenne lunghe e filiformi e ricorda i Cerambicidi. Oe. virescens Linn.

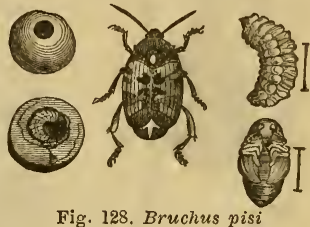

Fig. 128, Bruchus pisi

Molto importanti pei costumi (di cui ho già detto nel capitolo XIII) e per le applicazioni medicinali sono le specie della famiglia dei Meloidi. Il genere Meloë ha specie di mole me-

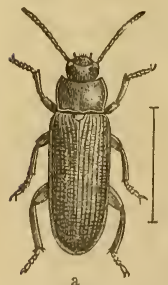

Fig. 129. Tenebrio molitor. $a$ insetto perfetto - $b$ larva.

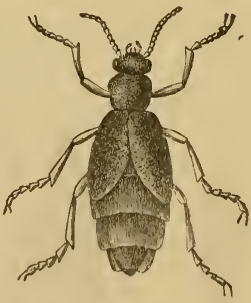

Fig. 130. Meloë proscarabaeus.

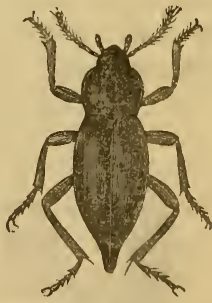

Fig. 131.

Blaps mortisaga.

diocre, di colore azzurognolo cupo più o meno spiccato, raramente con riflessi metallici. È specie comune fra noi la $M$. proscarabaeus Linn. Eleganti invece ed appariscenti sono i colori del genere Lytta Fabr. che comprende la comune cantaride. $L$. vescicatoria Linn., dal corpo di un bel color verde lucente (I).

(1) Intorno agli usi medici delle cantaridi, si consultino i trattati di zoologia medica, e fra gli altri quello già citato del Van Beneden. 
Il genere Sitaris Lat. ha il protorace quadrangolare, le elitre corte, strette e divaricate posteriormente, in modo che le ali rimangono allo scoperto. Questo genere presenta molto spiccato il fatto della ipermetamorfosi. S. humeralis Fabr.

Le specie del genere Mylabris Fabr. non sono rare fra noi e presentano il corpo ornato di eleganti macchiettature.

Del gruppo degli Eteromeri ricorderò ancora il genere Rhipiphorus Fabr. che ha le antenne del maschio con lunghe espansioni laterali disposte come le barbe di una penna. $R$. praeustus Gebl., non raro in Europa; il genere Mordella Linn., ricco di specie, dai colori neri, talvolta con riflessi argentei, e viventi sui fiori, $M$. fasciata Fabr., comune in Europa; il genere $A n$ thicus Payk., tipo della sotto famiglia degli Anticini, che comprende molte specie di coleotteri di piccola mole sparsi per tutta la terra; non è raro fra noi l'A. affinis Fabr.; il genere Tenebrio Linn., dal corpo allungato, subcilindrico, dai colori bruno neri e dalle larve allungate e vermiformi; la specie più nota è il $T$. molitor Linn. che vive nei nostri granai; il genere Blaps Fabr. che comprende coleotteri di mole relativamente grande, di color nero e che vivono nei luoghi oscuri ed umidi anche delle

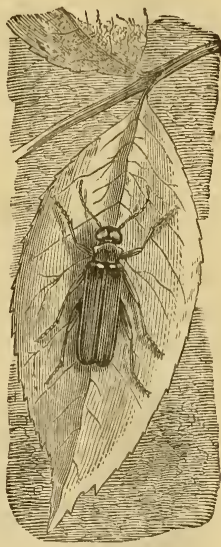

Fiz. 132.

Lytta vescicatoria. nostre case, $B$. mortisaga Linn., e finalmente il genere $P i$ melia Fabr. che presenta le elitre con rialzamenti cariformi più o meno spiccati, e talvolta ricoperte di tubercoli e di spine. Le specio di questo genere vivono per lo più nei luoghi sabbiosi ed aridi presso al mare. Non è rara la $P$. bipunctata Fabr.

Oltre ogni dire numerosi sono i generi e le specie appartenenti al gruppo dei Pentameri: io non accennerò qui, per le ragioni ripetutamente addotte, che qualche genere fra i più importanti.

Fra i Silofagi il genere Cis Latr. comprende specie di piccolissima mole e dal corpo cilindrico che vivono in varie specie di funghi. C. boleti Fabr. 
Anobium Fabr. Il corpo è cilindrico e di colore rossiccio, o bruno-nero. Le larve vivono nel legno. Una specie assai comune anche nelle nostre case, l'A. pertinax Linn., è notevole pel suo costume di simulare la morte quando viene molestato, e di percuotere, per richiamo sessuale, col capo le pareti delle gallerie che ha scavate nel legno in cui vive. I generi Ptinus e Ptilinus hanno costumi analoghi al precedente e comprendono specie che spesso sono dannosissime ai legnami da lavoro.

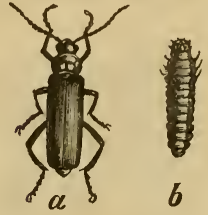

Fig. 133. Telephorus fuscus. $a$ insetto perfetto $-b$ larva.
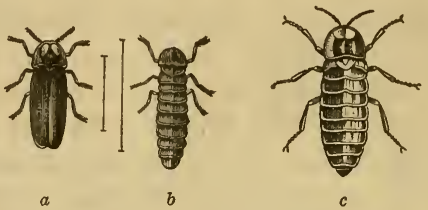

Fig. 134. Lampyris noctiluca. $a$ maschio $-b$ larva $-c$ femmina.

La famiglia dei Cleridi ha specie ornate per lo più di colori vivaci e di eleganti macchiettature, e che sono carnivore. Le larve del genere Trichodes Herbst, rappresentato da noi da due

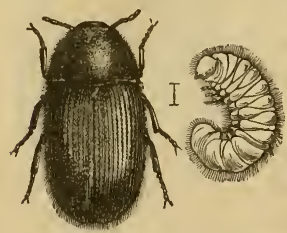

Fig. 135. Anobium paniceum. specie, $T$. apiarius Linn. e $T$. alvearius Fabr., vivono parassite negli alveari.

Fra i Malacodermidi ricorderò il genere Drilus Oliv., il quale presenta differenze sessuali spiccatissime; il maschio è alato ed ha antenne pettinate; la femmina invece è vermiforne ed attera e vive alle spese della Helix nemoralis. È specie comune fra noi il $D$. flavescens Rossi; il genere Telephorus Schäff, di cui una specie, il T. fuscus Linn., è comunissima in primavera sui fiori dei nostri prati ; il genere Lampyris Geoff. che ci presenta differenze sessuali analoghe a quelle del genere Drilus; il maschio cioè è alato, la femmina invece è vermiforme ed attera. Le specie di questo genere, come è noto a tutti, sono, generalmente, fosforescenti. Gli organi della fosforescenza sono collocati nell'addome e sono sviluppati principalmente nelle femmine (vedi cap. IV e V) (1),

(1) Le uova della L. noctituca sono, come venne osservato da qualche natura- 
e finalmente il genere Luciola Cast. in cui i due sessi sono alati e fosforescenti. È specie comune fra noi la $L$. italica Linn.

Gli Elateridi comprendono molte specie dannose a varie piante coltivate dall' nomo e specialmente il grano. Agriotes Eschsch. Le larve delle specie di questo genere sono dannossissime al grano (si consulti E. PERris « Sur les moeurs, ecc. des larves des Élatérides» Ann. soc. ent. de France, 1854). A. segetis Bjerk. Il genere Corymbites ha specie che vivono per lo più sui fiori e presentano colori metallici. C. cupreus Fabr.

Il genere Elater Linn., come viene inteso oggi, comprende specie ornate per lo più di colori assai vivaci ed eleganti che vivono nei vecchi tronchi degli alberi. Qualche specie fa vita

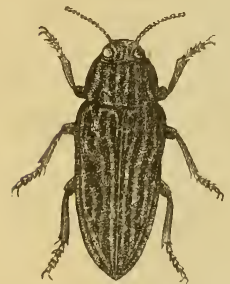

Fig. 136.

Buprestis mariana.

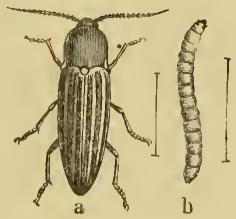

Fig. 137. Elater segetum. $a$ insetto perfetto $-b$ larva.

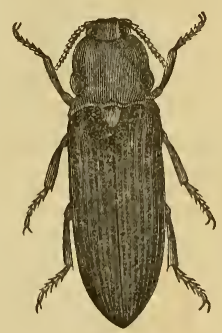

Fig. 138 .

Phyrophorus noctilucus.

notturna. Non è raro fra noi il bellissimo $E$. sanguineus Linn. dal protorace nero brillante e dalle elitre di color rosso cinabro vivacissimo. È pure degno di nota il genere Pyrophorus Illig. le di cui specie proprie della America hanno delle porzioni speciali del protorace che sono vescicolose e fosforescenti. I costumi di questi insetti non sono ancora ben noti. $P$. noctilucus Linn. (1).

Le specie costituenti la famiglia dei Buprestidi sono caratterizzate da un corpo allungato, largo anteriormente, molto

lista e come io stesso potei verificare, pure fosforescenti per un certo tratto di tempo dopo che sono stati emessi.

(1) Questa specie ed altre affini vengono nella Guadalupa chiamate Cucujos e si adoperano dalle creole come oggetto di ornamento. 
stretto posteriormente e dall'avere colori splendidissimi. Fanno parte di questa famiglia i più belli forse di tutti i coleotteri. Le forme più belle sono proprie delle regioni calde. Menzionerò il genere Trachys Fabr. dal capo piccolissimo e triangolare e che vive sulle foglie di piante. T. minuta Linn.; il genere Buprestis Linn. rappresentato fra noi dalla $B$. mariana Linn. di color bruno con riflessi di bronzo e velato leggermente di bianco che vive nei boschi di pini, ed il genere Dicerca Eschsch. di cui la D. aenea Linn. e la D. berolinensis Herbst. dal corpo color di rame e con bellissimi riflessi metallici e dall'integumento molto duro, non sono rari fra noi.

Fra i generi esotici che comprendono le specie più notevoli e per la mole e per la splendidezza dei colori menzionerò i seguenti : Catoxantha Sol. (Isole della Sonda, Molucche, Filippine), Euchroma Sol. (America meridionale e centrale), Polybothris Spinola (Madagascar), ecc.

I Lamellicornidi, che comprendono un grandissimo numero di forme, presentano differenze sessuali secondarie spiccatissime, forme strane e spesso colori assai eleganti.

I Lucanidi di cui è tipico il genere Lucanus Linn. sono rappresentati fra noi da pochi generi e da poche specie. È conosciuto da tutti il $L$. cervus Linn. o cervo volante.

Fra i generi esotici meritano speciale menzione i generi Chiasognathus Steph. dell' America meridionale, e Passalus Fabr. delle regioni equatoriali dei due continenti.

Le zampe anteriori foggiate a scavare caratterizzano i Copridi di cui molte specie hanno mole molto grande e colori eleganti. I Copridi vivono nei paesi caldi e depongono le uova in una pallottolina di escremento che essi stessi si fanno e che nascondono poi nella terra. Le forme più grosse appartengono all'Africa ed all'America.

Ricorderò l'Ateuchus sacer Linn. dell'Europa meridionale e dell'Africa settentrionale; il genere Sysiphus dalle zampe posteriori allungate; i numerosissimi Ontophagus che vivono negli escrementi dei ruminanti e dell'uomo stesso, e il Copris lunaris Linn. di color nero lucente, non raro fra noi.

Le specie del genere Aphodius, A. fossor Linn., e del genere Geotrupes, G. stercorarius Linn. hanno costumi simili a quelli dei Copridi. 
Il genere Hoplia è notevole per gli splendidi riflessi del suo corpo rivestito di squame come le ali delle farfalle. $H$. argentea Pz., ecc.

La Melolontha vulgaris Fabr., il comune maggiolino, specie tipica del genere Melolontha, è conosciuta da tutti. Questa

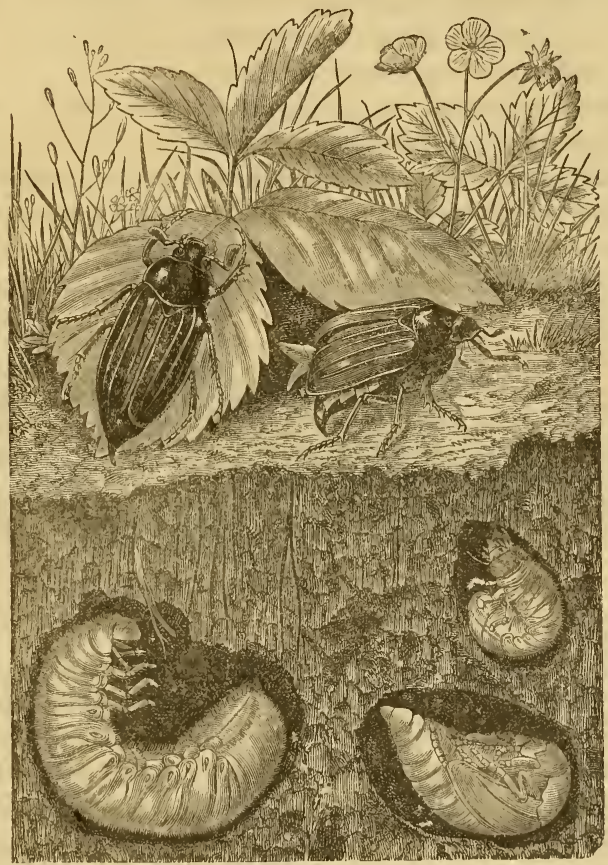

Fig. 139. Melolontha vulgaris. Superiormente due insetti perfetti, maschio e femmina: inferiormente a destra in basso una crisalide: le altre due figure rappresentano larve in vario grado di sviluppo.

specie è, sviluppandosi talvolta in grande numero, dannosissima a varie piante. Le larve, che nei primordi del loro sviluppo vivono in società, sono dannosissime rodendo le radici; gli insetti perfetti rosicano le foglie. Il ciclo di sviluppo pare sia fra noi, come in Francia, di tre anni. La M. hippocastani, simile alla precedente, è pure assai comune fra noi. 
Affini ai precedenti sono i generi Rhyzotrogus Latr. e Polyphylla Harr. Il primo comprende specie di piccola mole e simili ai maggiolini, il secondo specie di mole relativamente grande. Fra noi si trova, ma non è molto comune, la $P$. fullo Linn.

Anomala Sam. Una specie di questo genere, l' $A$. vitis (Fabr.) di un bel colore verde metallico, è nota pei danni, spesso assai gravi che arreca alle viti.

Affine alle Anomale ed alle Melolonte è il genere Geniates Kirb. dell'America meridionale.

I generi Dynastes, Oryctes, Megasoma, ecc., comprendono le forme più grosse di tutti i Lamellicorni e presentano differenze molto spiccate fra $i$ sessi. I maschi hanno quasi sempre corna più o meno spiccate.

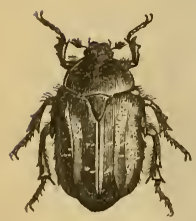

Fig. 140. Celonia aurata.

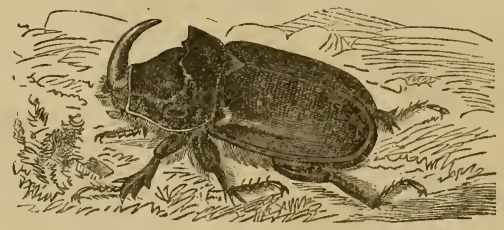

Fig. 141. Oryctes nasicornis maschio.

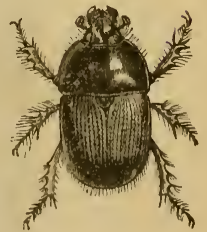

Fig. 142.

Geolrupes slercorarius.

È specie comune fra noi l'O. nasicornis Linn. Notissimo pure è il dinaste ercole (fig. 10) dell'America meridionale.

I colori eleganti, i riflessi metallici e l'integumento molto duro sono proprii dei Cetonidi che abbondano stranrdinariamente di specie e vivono principalmente nelle regioni calde. Sono pure note a tutti la C. aurata Linn., la C. (Oxythyrea Muls) stictica Linn., ecc. che vivono sui fiori.

Ricorderò di passaggio i Birridi dal corpo quasi sferico, Byrrhus gigas Fabr., i Dermestidi, di cui le specie dei generi Dermestes ed Anthrenus riescono spesso dannosissime alle raccolte di storia naturale, Dermestes lardarius Linn., Anthrenus museorum Linn.; i Criptofagidi che vivono per lo più nei funghi, Micetophagus quadripustulatus Linn.; i Nitidulidi dalla mole spesso piccolissima e che vivono nel legno marcio, sui funghi, sui cadaveri degli animali e sui fiori ; hanno colori 
giallastri o verdastri o neri, Nitidula bipustulata Linn., $N$. aenea, ecc.

La famiglia degli Isteridi comprende un grande numero di specie dal corpo ovaliforme ed appiattito e di color nero lucente con macchie di color rosso spiccato, che vivono nelle materie animali in decomposizione; qualche specie vive pure nei formicai. Hister cadaverinus Hoffm., H. stercorarius Hoffm.

Costumi simili a quelli della famiglia ora menzionata hanno i Silfidi, fra cui menzionerò il genere Silpha Fabr. che comprende numerose specie dal corpo appiattito e di colore nero opaco, qualche volta macchiettato di chiaro. S. obscura Linn., S. quadripunctata Linn. ed il genere Necrophorus Fabr. notevole pei suoi costumi. Le specie di questo genere, che hanno

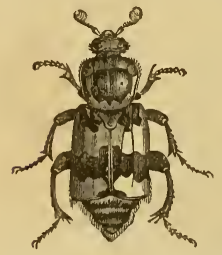

Fig. 143. Necrophorus vespillo.

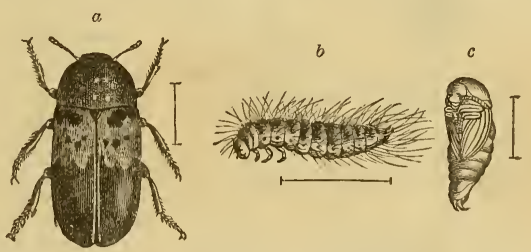

Fig. 144. Dermestes lardarius. $a$ insetto perfetto $-b$ larva $-c$ ninfa.

il corpo subcilindrico e tozzo; le zampe posteriori allungate, arcate ed atte a scavare, sono di color nero e giallo rossiccio; sotterrano i cadaveri dei piccoli mammiferi, nei quali depongono le uova. Le larve che si sviluppano si nutrono della carne che in questo modo le circonda. Sono specie non rare fra noi il $N$. humator Fabr. e il $N$. vespillo Linn.

Costituiscono la famiglia dei Pselafidi numerose piccole specie di coleotteri che hanno le elitre molto corte, le antenne claviformi e che vivono nei luoghi umidi, sotto la corteccia degli alberi, sotto le pietre e spesso assieme alle formiche. Pselaphus Heirei Herbst, Machaerites spelaeus Iacq. d. Val. Questa specie ed altre affini sono notevoli per la loro vita in fondo alle caverne e per avere occhi rudimentali o per non averne affatto secondo la profonditá e il grado di luce in cui vivono. Oltre ogni dire numerose sono le specie della famiglia degli 
Stafilinidi, la quale ha corpo allungato ed elitre cortissime, tantochè l'addome rimane quasi intieramente scoperto. La mole di questi insetti è per lo più piccolissima. Essi vivono nelle materie animali o vegetali in putrefazione. Molte specie sono parassite o meglio commensali delle formiche. Io mi limito a menzionare, per dare una idea della forma generale dei coleotteri di questa famiglia, lo Staphylinus erythropterus (fig. 145).

Gli Idrofilidi hanno antenne corte e claviformi; corpo con-

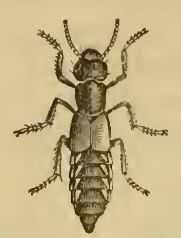

Fig. 145. Staphylinus erythropterus. vesso, ora di grandi, ora di piccolissime dimensioni. 亡 specie tipica l'Hydrophilus piceus Linn., comunissimo fra noi. Per cio che è del dimorfismo che si osserva nelle - femmine di questa specie vedi cap. V.

Il genere Dytiscus Linn. dal corpo allungato, ovaliforme ed appiattito è il tipo della famiglia dei Ditiscidi, famiglia la quale comprende insetti che vivono nelle acque dolci e che sono carnivori e predatori.

Qualche specie (Dytiscus latissimus, D. lapponicus) che

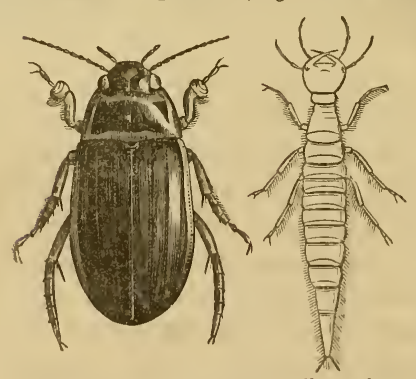

Fig. 146. Dytiscus marginalis colla sua larva sono proprie delle regioni settentrionali d'Europa si trovano pure, non molto frequentemente tuttavia, nei laghi delle alte regioni montagnose della Francia e del Piemonte. Altre specie, appartenenti soprattutto al genere Hydroporus, vivono pure nelle acque calde delle sorgenti termali.

Sono frequenti fra noi il D. marginalis Linn. di color verde bruno superiormente marginato di giallo, il Cybister roeselii Fabr. e l'Acilius sulcatus Linn. di color giallo bruno-chiaro e più piccolo dei precedenti.

La famiglia dei Girinidi racchiude poche specie che hanno corpo convesso, ovaliforme e che corrono, descrivendo tortuose curve, alla superficie delle acque. È noto a tutti il Gyrinus 
natator Linn. che è uno dei primi insetti che brillano al sole nelle pozze della nostra città al principio della primavera.

La numerosa ed elegante famiglia dei carabici chiude per noi l'ordine dei coleotteri. Questa famiglia comprende insetti dal corpo allungato e snello, dalle zampe e dalle mandibole molto sviluppate. I loro colori sono spesso assai vivaci ed eleganti. I Carabici sono sparsi per tutta la terra e sono, insieme coi Ditteri, gli insetti che si spingono più in alto sui monti. Ricorderò i generi seguenti.

Bembidium, tipo di un gruppo di piccoli carabici, alcuni dei quali sono cavernicoli e ciechi.

Anophtalmus Sturm, che è cieco e vive nelle caverne.

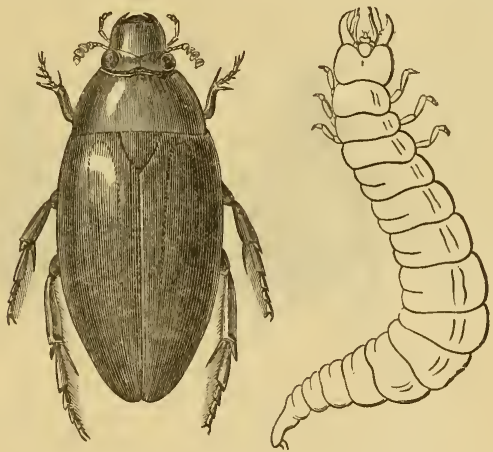

Fig. 147. Hydrophilus piceus colla sua larva.

Harpalus Latr. e Feronia Latr. generi ricchissimi di specie. H. ruficornis Fabr., F. metallica Fabr.

Scarites Fabr. che comprende specie dal capo grande e dalle mandibole robuste che abitano le regioni calde e sabbiose. S. Gygas Fabr.

Brachinus Weber. Questo genere è notevole per le esplosioni che molte specie possono produrre emettendo dall' ano dei gaz che hanno proprietà analoghe ai vapori dell'acido nitrico. B. crepitans Linn.

Carabus Linn. Gli insetti di questo genere, numerosissimi, abitano principalmente la regione paleartica. Sono scarsissimi o mancano al tutto nelle altre regioni. I Carabi hanno spesso 
elegantissimi colori (C. auronitens, C. rutilans, C. olimpiae, ecc.) ed amano principalmente i luoghi umidi delle regioni alpine. Sono voracissimi e carnivori, e possono perciò essere, entro certi limiti, di qualche utilità alla agricoltura. L'Italia ha molte specie di Carabi, alcune delle quali le sono proprie.

Cychrus Fabr. Questo genere è proprio quasi esclusivamente delle alte regioni alpine. C. italicus Bon.

Calosoma Weber. Questo genere ha costumi analogi a quelli del genere Carabus. Le ali membranose sono ben sviluppate. Le Calosome sono utili all' agricoltura in quantochè distruggono molti bruchi soprattutto del Bombyx processionea

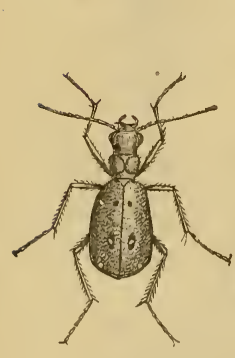

Fig. 148.

Cicindela campestris.

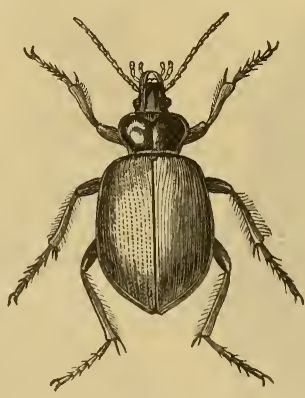

Fig. 149.

Calosoma sycophanta.

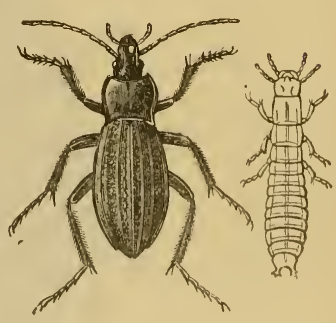

Fig. 150.

Carabus cancellatus colla sua larva.

Linn. È comune fra noi la $C$. sycophanta Linn. dal protorace nero e dalle ali di un bel verde metallico con riflessi porporini.

Omophron Lat., Il corpo è ovaliforme, appiattito e ricorda nell'aspetto gli Idrocantaridi. L'O. limbatum Fabr. vive nelle sabbie lungo i fiumi.

Mormolyce Hagenb. Questo genere curiosissimo, che ha lo ali allargate in modo da dare all'animale l'aspetto di una foglia, comprende al giorno d'oggi tre specie che vivono nelle Indie orientali. La più anticamente nota è il $M$. phyllodes Hag. di Giava.

Cicindela Linn. Le cicindele sono i più belli di tutti i carabici e per la snellezza delle loro forme e per la vivacità dei 
loro movimenti. Vivono nei luoghi caldi e sabbiosi e volano e corrono nelle giornate in cui più è caldo e splendido il sole (vedi intorno ai costumi della larva della C. Campestris Linn., cap. IV). È comune fra noi in primavera anche nell' interno delle città la $C$. campestris Linn. Non sono rare pure la $C$. hybrida, la C. germanica, e sui monti la $C$. chloris.

Manticora Fabr. Questo genere proprio dell' Africa meridionale comprende le specie più bellicose di tutti i carabici. M. maxillosa Fabr. (1).

(1) La caccia delle Manticore non è molto facile e la manticora molte volte si rivolta e cerca di opporre resistenza colle robuste ed aguzze mandibole. Riferirò a questo proposito le parole seguenti del Castelnau: "Une autre fois je vis une Manticore courir vers moi avec la rapidité ordinaire des mouvements de ces animaux; mais, en m'apercevant, elle s'arrêta tout-à-coup et dirigea sa marche de manière a former un angle droit avec la direction qu'elle suivait précédemment; tout-à-coup, ayant rencontré un monticule de sable, elle s'adossa contre et se mit sur la défensire. J'allais presque la saisir, lorsque j'aperçus, a 2 ou 3 mètres de moi, un cobracapel, le plus dangereux reptile de l'Afrique australe, qui évidemment lui donnait la chasse; me trouvant sans armes, je me retirai rapidement et laissai ma brave Manticore se débattre contre son terrible adversaire ". 


\section{Bibliografia.}

DejEan. - Iconographie et histoire naturelle des Coléoptères d'Europe. Paris, 1829-36.

- Catalogue des Coléoptères. Paris, 1837.

- Species général des Coléoptères. 1825-1838.

F. Stein e J. Weise. - Catalogi coleopterorum Europae. Berlino, 1877.

L. Fairmaire e A.LaboulbÈne. - Faune entomologique française - Coléoptères. Paris, 1854.

A. Marseur. - Catalogue des Coléoptères d'Europe, ecc. Paris, 2a ed., 1863.

Gemminger e Harold. - Catalogus Coleopterorum. Monaco, 1868-77.

Erichson, Schadi, Kiesenwetter e Kraatz. - Naturgeschichte der Insecten.

Deutschlands, 1848-68.

Redtenbacher. - Fauna austriaca. Vienna, 1873.

LAcokdaire. - Genera des Coléoptères. Parigi, 1854-75.

- Monographie des Coléoptères subpentamères de la famille des Phytophages, 1845.

E. Mulsant. - Histoire naturelle des Coléoptères de France, 1839-78.

A. Fadvel. - Species de la faune gallorhenane. Cołéoptères. Caen, 1868-75.

C. J. Sснӧмнеr. - Genera et species curculionidum. Parigi, 1833-44.

A. Gerstaecker. - Rhipiphoridum Coleopterorum familiae dispositio systematica. Berolini, 1855.

E. CANDEZE. - Monographie des Elatérides. Liège, 1857.

Erichson. - Genera et species Staphylinorum. Berolini, 1840.

Gory et Percheron. - Monographie des Cétoines. Paris, 1833.

Bonelli. - Observations entomologiques. Mem. dell' Accad. delle Scienze di Torino, 1809.

Thompson. - Monographie des Cicindélides. Paris, 1857.

0. Pirazzoul. - Cicindele italiane. Bull. della Soc. Ent. Ital., vol. IV, 1872.

- I Carabj italiani, ibid., vol. III, 1871.

I. SturM. - Deutschlands Fauna. Nürnberg, 1805-57. 


\section{INENOTTERI}

Insetti con apparato boceale foggiato per masticare e per lambire: con quattro ali membranose; con metamorfosi compiute.

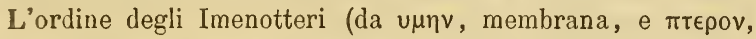
ala), creato da Linneo, comprende i più elevati fra tutti gli insetti. Gli Imenotteri presentano una vita di relazione molto estesa ed è impossibile non riconoscere in essi, studiando i loro interessantissimi costumi, qualche cosa più che non il semplice istinto. Sarebbero necessarie molte e molte pagine per dare anche un solo profilo un po' esatto dei costumi delle principali specie di Imenotteri. Io non posso naturalmente nei limiti del presente libro estendermi molto intorno a questo argomento, il quale del resto venne trattato largamente da molti autori.

Il capo è sempre ben sviluppato e per lo più separato dal torace da uno stretto collo. In qualche caso (Cryptocerus atratus Latr., ed altri Formicidi) il capo porta delle spine e dei prolungamenti speciali. Le antenne sono in generale allungate, ora filiformi, ora ingrossate a clava verso l'apice, ora dentate, ora, raramente, pettinate. L'apparato boccale è foggiato, come abbiamo già visto (Cap. XI), ora per masticare, ora per lambire. Il torace per lo più è 0 arrotondato e convesso o alquanto allungato: in qualche caso (Thoracantha latreillei Guer.) si sviluppa moltissimo e si prolunga posteriormente sull'addome. Nei generi Cryptocerus ed Atta, ecc., il torace presenta dei prolungamenti laterali spiniformi corrispondenti a ciascun segmento. Il mesotorace porta, superiormente alla base delle ali, due piccole squame mobili (squamulae, tegulae).

Le ali sono quattro ed in generale ben sviluppate, talvolta nelle specie sociali mancano in uno dei sessi e negli operai. 
Le ali anteriori sono più sviluppate delle posteriori; sia le une, sia le altre hanno poche nervature. Notevole per la forma delle sue ali è il piccolissimo Mymar pulchellus Walk. in cui le ali anteriori sono fatte a spatola molto allungata e sono orlate di lunghi peli e le posteriori sono quasi intieramente atrofizzate e ridotte a due esili filamenti.

Le ali degli Imenotteri presentano spesso dei colori iridati elegantissimi dovuti a fenomeni di interferenza luminosa.

Le zampe sono ben sviluppate, talvolta (genere Chalcis) le posteriori sono molto ingrossate. Ora sono prive di peli, ora invece sono pelose e in qualche caso le posteriori portano delle spazzolette di peli molto sviluppate (generi Centris, Acanthopus, ecc.). I tarsi hanno ordinariamente cinque articoli, il primo dei quali è lungo e grosso.

Oltre ogni dire variabile è la forma dell'addome il quale per lo più è unito al torace per mezzo di un peduncolo. I casi in cui l'addome è sessile sono relativamente rari (generi Cimbex, Cladius, Xyela, Sirex, ecc.). Il peduncolo può essere molto lungo e l'addome al contrario molto corto e piccolo come nei generi Evania, Faenus, Ammophilus, ovvero relativamente corto, mentre l'addome è invece molto grande come nei generi Xylocopa, Megachile, ecc. L'addome poi ora è grande ed ovaliforme (generi Cimbex, Schizocera), ora è cilindrico, sottile e più o meno allungato (generi Ophion, Pelecinus). Nel $P$. polycerator Latr. l'addome ha una lunghezza eguale a tre o quattro volte quella del corpo dell'insetto (Stephanus, ecc.). L'addome può pure essere, ora piriforme (Synagris), ora goccioliforme (Ammophilus, ecc.). Non raramente l'ultimo segmento dell'addome porta due o più filamenti spesso Iunghissimi (Stephanus, Vipio). Generalmente gli Imenotteri hanno un ovopositore (terebra) e spesso anche un pungiglione velenifero.

Il sistema nervoso negli Imenotteri è ben sviluppato e presenta un grado relativamente grande di centralizzazione. I gangli toracici sono due; sono cinque o sei gli addominali. Gli Imenotteri, di cui vennero descritte molte migliaia di specie, non giungono mai a grande mole: molti sono minutissimi e vivono parassiti di un grande numero di altre specie appartenenti ai vari ordini d'insetti. Per questo rispetto gli Ime- 
notteri tornano utili all'uomo (Cap. II). Le larve vivono o nel corpo di altri animali, o nei tessuti vegetali. In vari casi si ha una pseudoninfa, stadio che precede immediatamente quello di ninfa. Moltissime larve di Imenotteri tessono un bozzolo, che non raggiunge tuttavia quasi mai la finezza di quelli dei Lepidotteri, in cui compiono il loro sviluppo. Non poche specie, come tutti sanno, costruiscono nidi in cui vivono allo stato sociale un certo numero di individui. Dei costumi io darò qualche cenno parlando delle specie di Imenotteri più importanti. Gli Imenotteri hanno spesso colori assai belli (Chrysis), sono sparsi per tutta la terra, abbondando naturalmente nelle regioni equatoriali: vivono ad altezze relativamente grandi sui monti.

\section{Tavola di classificazione degli Imenotteri.}

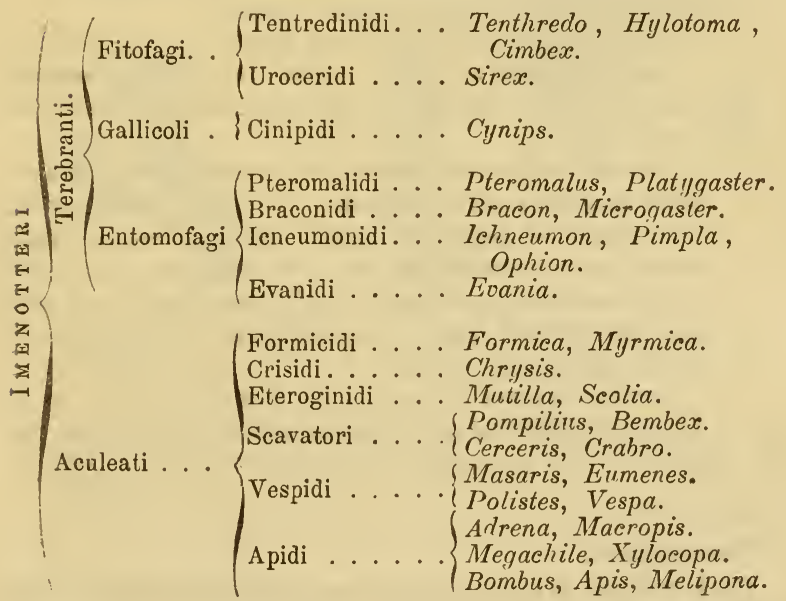

A. Le femmine hanno un ovopositore ma non portano pungiglione velenifero Terebranti. a. Addome sessile . . . . . Fitofagi.

$\alpha$. Larve simili ai bruchi, provviste di ano.

a. Ovopositore delle femmine in generale corto: antenne ingrossate verso l'apice . . . Tentredinidi. 
$a^{\prime}$. Ovopositore delle femmine in generale lungo: antenne filiformi

Uroceridi.

$\alpha^{\prime}$. Larve apode, sprovviste di ano . . Gallicoli.

a. Antenne lunghe filiformi : le femmine pungono le piante e, versando un liquido irritante nella ferita, producono delle escrescenze particolari dette galle. Cinipidi. $a^{\prime}$. Addome peduncolato

Entomofagi.

a. Antenne piegate ad angolo: ali anteriori con una sola nervatura marginale anteriore e senza nervature ricorrenti.

Pteromalidi.

$a^{\prime}$. Antenne allungate: ali anteriori con una sola nervatura ricorrente

Braconidi.

$a^{\prime \prime}$. Antenne allungate: ali anteriori con due nervature ricorrenti

Ichneumonidi.

$a^{\prime \prime \prime}$. Antenne allungate: addome piccolo e lungamente peduncolato: ali posteriori quasi al tutto prive di nervature.

Evanidi.

$A^{\prime}$. Le femmine hanno un pungiglione velenifero.

a. Antenne piegate ad angolo: vita sociale. Formicidi.

$a^{\prime}$. Antenne piegate ad angolo: colori vivaci, di un bel rosso azzurro o verde metallico: palpi mascellari con cinque articoli

Crisidi.

$a^{\prime \prime}$. Antenne lunghe nel maschio, corte invece nella femmina: palpi mascellari con sei articoli . . Eteroginidi.

$a^{\prime \prime \prime}$. Antenne non piegate ad angolo, allungate: zampe lunghe e con spine e pungiglioni : fanno vita solitaria. Scavatori. $a^{1 \mathrm{v}}$. Antenne piegate ad angolo: palpi mascellari di sei articoli: ali anteriori piegate longitudinalmente: fanno vita sociale e fabbricano un nido . . . Vespidi.

$a^{v}$. Antenne piegate ad angolo: ali anteriori che non si ripiegano longitudinalmente: primo articolo dei tarsi con una spazzoletta di peli : fanno vita ora solitaria ora sociale.

Apidi.

\section{Tentredinidi.}

Gli Imenotteri di questa famiglia sono caratterizzati dall'avere un corpo grosso e tozzo, antenne non piegate ad angolo e di un numero di articoli variabile da nove a quattordici. Molti sono i generi che formano questa famiglia e si distinguono fra loro principalmente per la nervatura delle ali. Le larve si nutrono delle foglie di varie specie di piante, vivono in societå per un certo tratto di tempo, e si fabbricano un bozzolo nel 
quale passano lo stadio di ninfa. Qualche specie riesce dannosa a varie piante.

Tenthredo Linn. Le specie di questo genere hanno per lo più le antenne di nove articoli e le mandibole dentate internamente. Le numerose specie di questo genere vivono sopra varie sorta di piante; cosi la $T$. scrophulariae vive sulla Scrofularia, la T. scalaris Klug. vive sul salice, ecc.

Le specie del genere Gladius Ill. hanno antenne di nove articoli, spesso pettinate nei maschi. Il $G$. difformis Latr. di color nero e colle zampe chiare, vive nei rosai. In qualche specie

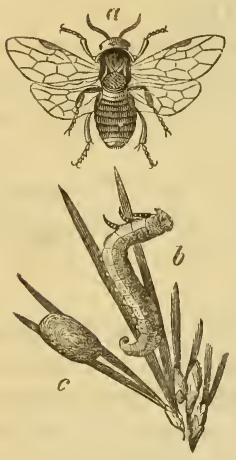

Fig. 151. Tenthredo (Lophyrus) pini. $a$ insetto perfetto $-b$ larva $c$ crisalide.

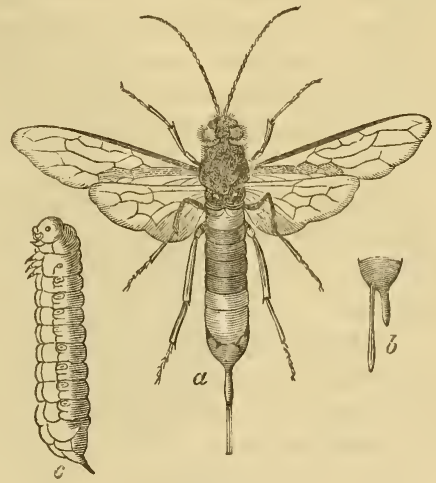

Fig. 152. Sirex gigas.

$a$ insetto perfetto - $b$ estremità dell'addome vista di profilo - c larva.

di questo genere vennero osservati casi di partenogenesi. $H y$ lotoma Fabr. Le antenne nelle specie di questo genere sono alquanto ingrossate verso la loro estremità superiore. La specie più nota è l'Hylotoma rosae Fabr., di cui la larva si nutre delle foglie delle rose.

Il genere Cimbex Oliv. è ricco di specie dal corpo grosso e robusto e dalle antenne corte e ingrossate alla loro estremità superiore. Le larve vivono sopra varie sorta di piante, e passano lo stadio di ninfa in un bozzolo che esse intessono fra i rami. C. femorata Linn.

Il genere Perga Leach. è affine al genere precedente, com- 
prende specie di mole relativamente grande ed è proprio della Nuova Olanda.

Nella famiglia degli Uroceridi ricordiamo il genere Cephus Fabr. dal corpo allungato e sottile, dalle antenne lunghe e filiformi. Le specie di questo genere vivono nell'interno dei fusti di varie sorta di piante. Il C. pygmaeus vive nel fusto del grano e spesso lo danneggia in modo assai notevole. Un'altra specie il C. compressus abita i giovani rami dei peri.

Degno di menzione è pure il genere Sirex che comprende specie di mole relativamente grande e che hanno l'addome allungato e cilindrico. La femmina ha un ovopositore diritto, robusto ed atto a forare i legni più duri (1).

Fra le numerose specie di questo genere ricorderò la S. gigas Linn. che si trova principalmente nei boschi di conifere, e la S. juvencus Linn. che ha costumi analoghi alla specie precedente.

Il genere Cynips Linn., tipo del gruppo degli Imenotteri gallicoli, comprende specie che si sviluppano nelle escrescenze o galle che le femmine producono colla loro puntura sopra varie parti dei vegetali.

I caratteri del genere Cynips sono: antenne diritte filiformi, di quattordici articoli (nelle femmine spesso sono più corte e più grosse che non dei maschi): torace bitorzoluto e peloso: ali anteriori con poche nervature: ovopositore finissimo ed avvolto a spira nell'addome. Il $C$. quercus-folii Fabr., il $C$. gallae-tinctoriae Oliv. che produce la galla conosciuta in commercio col nome di galla di levante, ecc., sono le specie più note e più importanti. Sono generi affini al precedente $\mathrm{i}$ seguenti: Rhodites Hrtg. (Rh. rosae Linn.), Figites Latr. ( $F$. scutellaris Latr. parassita delle larve di Sarcophaga), Ibalia (I. cultellator Latr.).

(1) Il maresciallo Waillant mostrò nel 1857 all'Accademia di Francia vari pacchi di cartuccie di cui le palle erano state forate da parte a parte durante il soggiorno delle truppe francesi in Oriente per la guerra di Crimea. Il Dumeril riconobbe che i fori erano stati fatti dal Sirex juvencus Linn. e credette fossero le femmine che avessero fatto ciò coi loro ovopositori. Pare tuttavia più probabile l'ipotesi di G. Méneville, il quale crede siano invece autrici dei fori sopradetti le larve della Sirex citata, le quali hanno mandibole robustissime. Quest'ultima ipotesi è stata confermata posteriormente da altri fatti consimili. 
Pteromalus Swed. Il capo ed il torace sono ricoperti più o meno fittamente di peli: le antenne hanno tredici articoli $e$ sono filiformi nei maschi, ingrossate invece, per lo più, a clava nelle femmine.

Le larve delle specie di questo genere riescono utilissime all'uomo inquantochè esse vivono alle spese di un grande numero di bruchi (vedi capitolo II). Sono specie note e frequenti il $P$. puparum Fabr. e il P. larvarum Fabr. I generi Pla-

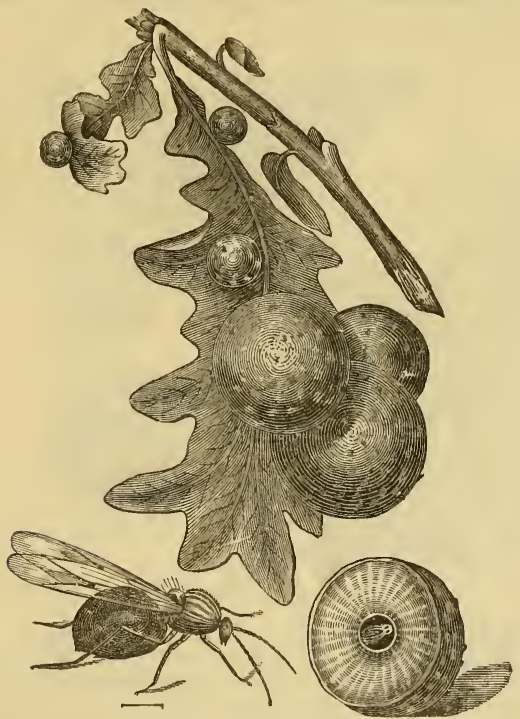

Fig. 153. Cynips quercus-folii - Superiormente una foglia con varie galle, inferiormente una galla sezionata con una ninfa nel mezzo.

tygaster Latr., Teleas Latr., Eurytoma Ill., ecc. sono affini al precedente.

La famiglia dei Braconidi, di cui sono tipici i generi $\mathbf{M i}$ crogaster Latr. e Bracon Fabr., comprende specie che hanno costumi non molto diversi da quelli dei Pteromalidi. Nel genere Microgaster le antenne sono allungate e di diciotto articoli. Il $M$. glomeratus è parassito dei bruchi di molte specie di Lepidotteri. 
La famiglia degli Ichneumonidi comprende un grande numero di generi e di specie, spesso di piccola mole, il cui studio è assai difficile e i di cui costumi sono simili a quelli dei Pteromalidi. Gli Ichneumonidi sono parassiti di un grande numero di insetti e soprattutto dei bruchi dei Lepidotteri. Il genere Ichneumon Grav. ha l'addome peduncolato e allungato; l'ovopositore è nascosto. I. incubitor Linn. Il genere Pimpla Fabr. comprende specie dal corpo allungato, dalle antenne sottili e lunghe e dall'ovopositore pure assai lungo. $P$. manifestator

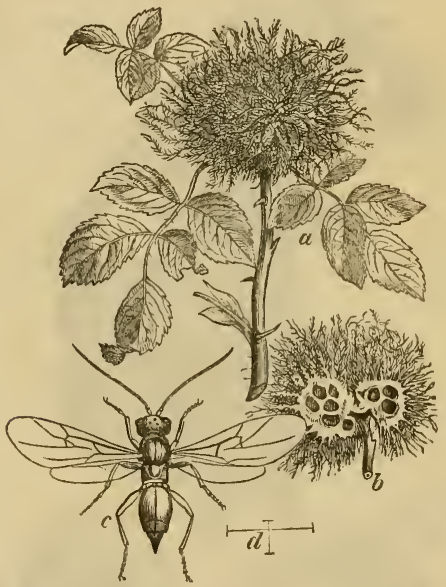

Fig. 154. Rhodites rosae $-a$ galla $-b$ galla sezionata $-c$ insetto $d$ linee indicante la grandezza naturale dell'insetto.

Linn. parassita allo stato di larva di varie sorta di bruchi. Il genere Ophion Fabr. merita di essere menzionato per le sue antenne che hanno più di sessanta articoli. O. luteus Linn.; le uova sono peduncolate. Le specie di questo genere sono come quelle del genere precedente parassite di vari bruchi.

Facilmente riconoscibile, per l'addome piccolo, lungamente peduncolato ed articolato coll'orlo anteriore del metatorace, è il genere Evania Latr. Una specie l'E. appendigaster Latr. si trova non raramente nelle case stesse dell'uomo ed è parassita, a quanto pare, delle blatte. 


\section{Aculeati.}

La presenza di un pungiglione velenifero nella femmina e la vita sociale di molte specie caratterizzano questo gruppo di Imenotteri. Io non posso qui descrivere minutamente i costumi interessantissimi delle specie di questo gruppo, ciò sarebbe troppo lungo. Io non faccio che ricordare le cose più spiccate.

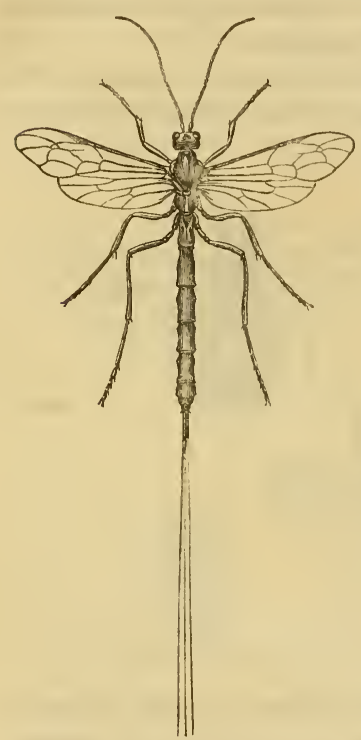

Fig. 155. Pimpla manifestator.

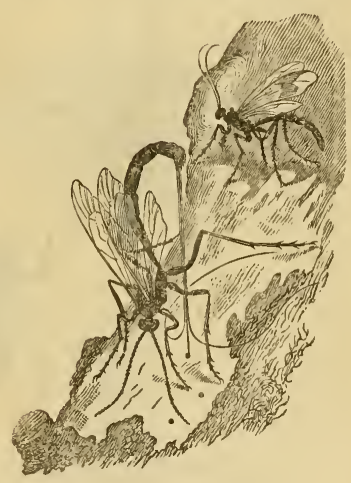

Fig. 156. Pimpla manifestator in atto di deporre le nova: superiormente il maschio.

Chi desidera più minuti ragguagli ricorra alle opere citate nella bibliografia posta in fondo al capitolo.

I Formicidi hanno le antenne piegate ad angolo: le mandibole robuste e gli individui vivono in società composte di varie forme ben spiccate, vale a dire: maschi, femmine alate, operaie e soldati privi di ali. Le operaie non sono altro che femmine abortite. I formicidi scavano lunghe e spesso molto complicate gallerie o nel terreno o nei tronchi degli alberi. Giunta la buona stagione i maschi e le femmine escono a volo dal formicaio e si accoppiano. Il maschio muore, la 
femmina invece perde le ali e ridiscende nel formicaio a deporvi le uova. Le formiche non ammassano nei nostri paesi provvigioni per la stagione invernale, passando esse in letargo questo periodo dell'anno. I Formicidi sono principalmente numerosi nelle regioni equatoriali. Nel Brasile ve ne hanno molte specie, alcune delle quali di mole relativamente grande, compiono, riunite in grande numero, lunghi viaggi distruggendo tutto quanto incontrano sul loro passaggio.

Accenneró qui di sfuggita il fatto non ancora ben conosciuto di molte specie di altri insetti che vivono probabilmente come

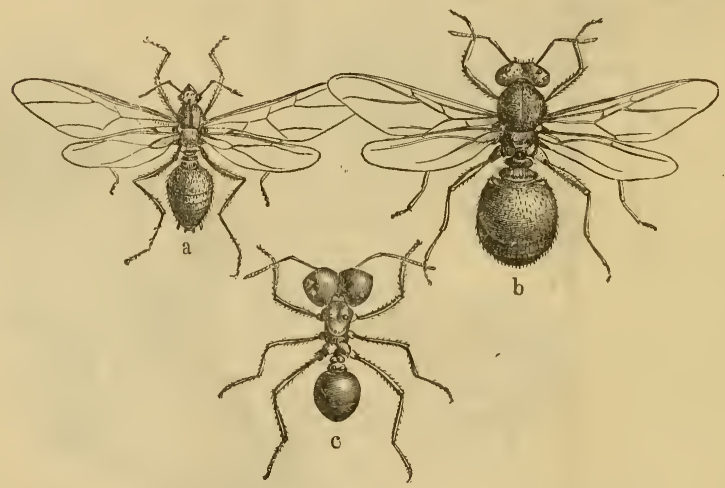

Fig. 157. Alta cephalotes - $a$ maschio $-b$ femmina $-c$ operaia

commensali nei formicai in buona armonia colle formiche stesse. I Formicidi sono voracissimi e si nutrono di sostanze animali e vegetali; sono ghiottissimi dei sughi zuccherini. Molte specie mordono e pungono dolorosissimamente. Molte specie pure preparano un liquido acre conosciuto col nome di acido formico. Ricorderò i generi seguenti: Formica Linn. privo di pungiglione, F. rufa Linn.; Myrmica Latr. provvisto di pungiglione, $M$. graminicola Latr., M. acervorum Fabr.; Atta Fabr., A. cephalotes Fabr. dell'America meridionale, una delle specie che compiono i viaggi sopradetti; Cryptocerus Latr. che abita in gallerie scavate nei rami degli alberi.

I splendidi colori, rosso, azzurro e i riflessi metallici fanno riconoscere a primo colpo d'occhio gli Imenotteri appartenenti 
alla famiglia delle Crisidi. In questa famiglia le antenne sono piegate ad angolo, e l'addome è peduncolato. I costumi delle Crisidi sono molto notevoli e non ancora ben noti. Molte specie depongono le uova nei nidi di varie sorta di altri Imenotteri (Apis, Crabro, Cerceris, ecc.), e adoperano in questa operazione molte astuzie; talvolta impegnano anche aspri combattimenti coi legittimi proprietari dei nidi. È specie non rara la $C$. ignita Linn. che ha il torace di color verde-azzurro e l'addome rossofuoco vivissimo. È pure non raro fra noi lo Stilbum calens.

Le specie della famiglia degli Eteroginidi sono caratterizzate principalmente da numerose e spiccate differenze sessuali.

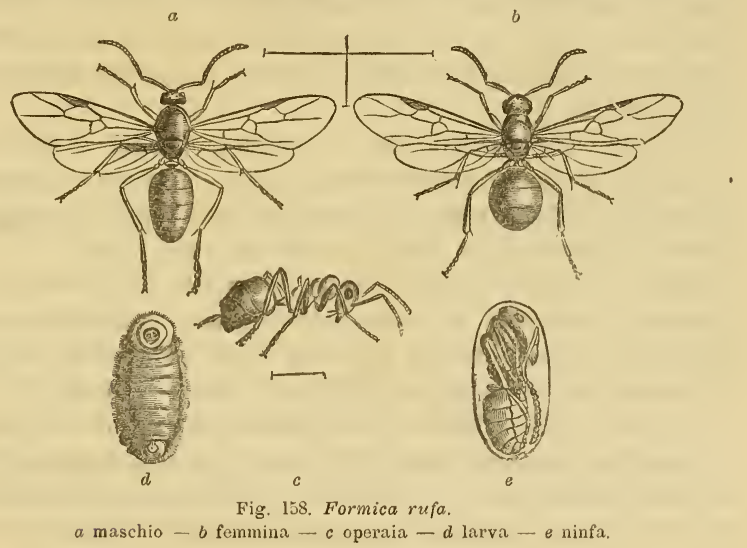

Le femmine sono spesso prive di ali, ed hanno le antenne molto più corte che non i maschi. Le specie di questa famiglia, come le Crisidi, depongono le uova nei nidi di altri Imenotteri. Ricorderò i generi; Mutilla Linn. in cui le femmine sono attere ed hanno i segmenti toracici saldati e l'addome ovale ed allungato, $M$. calva Fabr., il maschio in questa specie è intieramente nero, la femmina invece ha la sommità del capo e il torace rosso e i margini dell'addome grigiastri; Scolia Fabr. questo genere comprende un numero grandissimo di specie in cui la femmina è alata; la $S$. hortorum Fabr. non è rara fra noi e vive nello stato di larva 
parassita della larva dell'Oryctes nasicornis, la $S$. bicincta Fabr. fa dei fori profondi nella sabbia e presenta costumi simili a quelli delle Cerceris, raduna cioè nei nidi varie specie di Ortotteri affinchè servano di cibo alle larve che sbuccieranno dalle uova.

Gli Scavatori sono caratterizzati dallo scavare che fanno le femmine lunghe gallerie nella sabbia, nella terra, od anche nel legno secco. La femmina depone in queste gallerie le uova ed ha cura di ammucchiare intorno a queste una notevole quantità di nutrimento destinato alle larve appena saranno uscite dall'uovo. Gli insetti che sono portati nel nido non vengono uccisi, ma soltanto hanno paralizzati tutti i movimenti in seguito alla puntura che vien fatta loro dall'Imenottero nella catena gangliare ventrale. Si consulti intorno a ciò gli scritti del Fabre (1).

Il genere Pompilius Latr. ha antenne lunghe e filiformi. Le specie di questo genere sono agilissime e vivono nei tronchi secchi degli alberi: nutrono le loro larve con ragni. Sono specie note il $P$. variegatus Linn. e il $P$. viaticus Linn.

Bembex Fabr., antenne piegate ad angolo: mascelle e labro inferiore prolungati in una specie di beeco: mandibole acute e falcate. La $B$. rostrata Linn. fa il suo nido nella sabbia e vi ammucchia varie specie di Ditteri (Sirfidi e Muscidi).

Il genere Cerceris Latr. ha le antenne claviformi e le zampe spinose. Le numerose specie di questo genere si scavano i nidi nei luoghi sabbiosi e vi radunano varie specie di Coleotteri. La C. bupresticida predilige essenzialmente i Coleotteri della famiglia dei Buprestidi.

Crabro Fabr. Le antenne sono piegate ad angolo, fusiformi nei maschi, filiformi invece e un po' ingrossate al loro apice nelle femmine. Le specie di questo genere abitano principalmente le regioni calde e spesso fanno il nido nel legno tarlato e vi raccolgono Ditteri, larve di Lepidotteri (Tortrix), ecc. Le ultime due famiglie degli Imenotteri, Vespidi e Apidi comprendono le forme più perfette, morfologicamente e per

(1) Observations sur les moeurs des Cerceris. Ann. des Scienc. Nat., $4^{a}$ serie, vol.IV. Recherches sur quelques points de l'histoire des Cerceris de Bembex, etc. Ibid., 1856. 
l'estesa vita di relazione che presentano, di tutti gli Imenotteri e forse anche di tutti gli insetti.

Io non do qui che qualche cenno intorno ai costumi di questi insetti, imperocchè sarebbe troppo lungo l'esporli minutamente. $\mathrm{Si}$ consulti perciò qualcuno degli estesi trattati di entomologia da me ripetutamente citati e i trattati speciali d'apicoltura.

Il genere Masaris Fabr. (M. vespiformis Fabr.) ha costumi analoghi a quelli delle Cerceris.

Le specie che appartengono al genere Eumenes Fabr. hanno l'addome fatto a goccia e lungamente peduncolato. Qualche specie nutre le larve con miele, qualche altra invece con bruchi.

Molto notevoli pei costumi sono le specie del genere Polistes Fabr. In questo genere troviamo già il fatto del polimorfismo dipendente dalla vita sociale. Il Siebold crede che questa specie non partorisca dapprima che femmine, le quali poi non fecondate dànno origine partenogeneticamente a maschi. La $P$. gallica Linn. di color nero con macchie gialle è specie comune in Europa e costruisce un nido in cui le celle sono prive di involucro comune. Ricorderò ancora la Chartegus nitidulans Fabr. che vive nella Caienna e che fa il nido con una sostanza simile per l'aspetto e per la consistenza al cartone e la C. brasiliensis Latr. che, come ha constatato A. Saint-Hilaire, produce un miele che ha proprietà venefiche e.che in qualche caso può produrre la morte dell'uomo.

Del genere Vespa Linn. menzionerò il comune calabrone (Vespa crabro Linn.) noto per le sue dolorosissime punture, e che ha addome ad un dipresso cilindrico e corpo di color rosso ferruginoso con macchie chiare e scure.

La famiglia degli Apidi comprende un grandissimo numero di specie che, o vivono solitarie, o allo stato sociale, che sono sparse per tutta la terra $\theta$ che presentano i seguenti caratteri generali : antenne dei maschi più grosse e più corte che non quelle delle femmine: tibie e tarsi soprattutto delle zampe posteriori allargate e rivestite in parte da spazzolette di peli: corpo coperto di peli: varie specie presentano il fenomeno della partenogenesi: molte specie si costruiscono un nido, altre depongono le uova in quelli delle altre Api; si nutrono di polline e di miele.

Megachile Latr. Le zampe posteriori sono semplici e non 
atte a raccogliere il polline: addome con peli destinati a ritenere il polline. Le specie di questo genere si fabbricano nidi
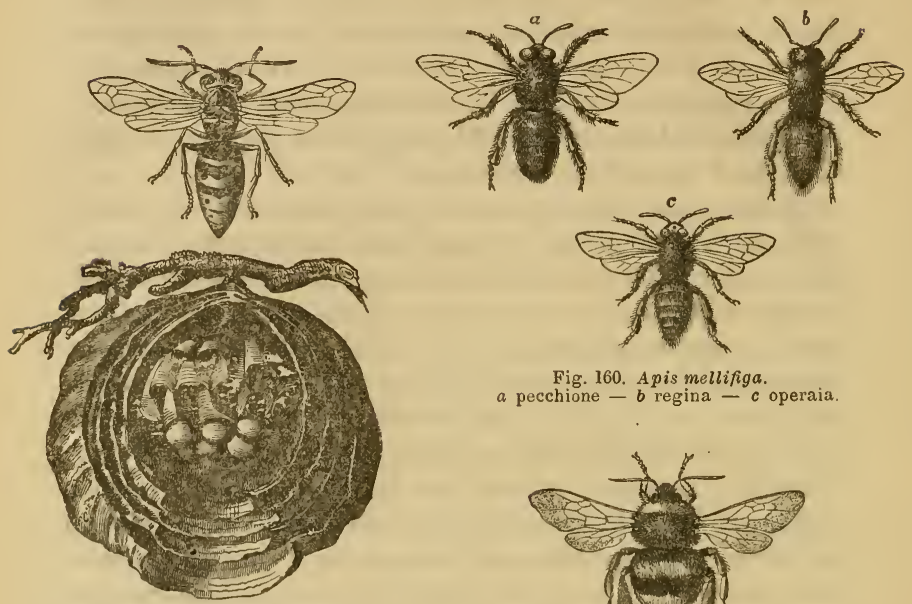

Fig. 159. Vespa vulgaris col suo nido.

Fig. 160. Apis mellifiga.

$a$ pecchione $-b$ regina $-c$ operaia.

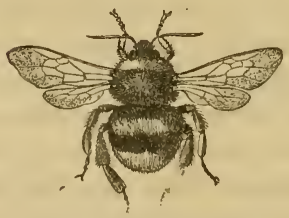

Fig. 161. Bombus terrestris.

in cui le cellule hanno la forma ad un dipresso di un ditale, ora le cellule sono fatte di terra, ora di foglie, ora di petali,
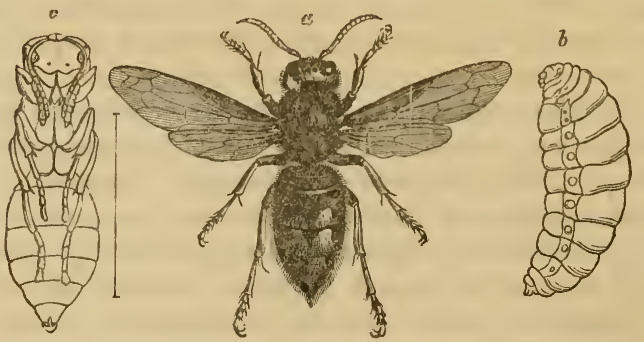

Fig. 162. Vespa crabro $-a$ insetto perfetto $-b$ larva $-c$ crisalide.

tagliati dalla larva in un modo speciale. M. muraria Fabr., M. (Authocopa) papaveris, ecc. 
Il genere Xylocopa Fabr. comprende molte specie in generale di grande mole che abbondano principalmente nell'America meridionale, nell'Africa e nelle Indie orientali. Le specie di questo genere scavano il loro nido nel legno tarlato. In Europa si trova non rara la $X$. violacea Fabr.

Bombus Latr. Le specie di questo genere sono facilmente riconoscibili pel loro corpo rivestito da lunghi e folti peli e pel loro grosso e largo addome. I nidi sono collocati per lo

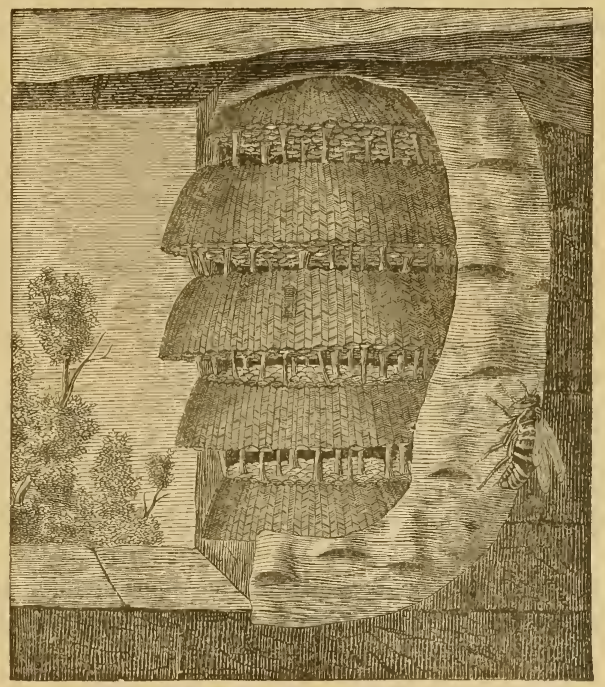

Fig. 163. Nido di vespa Crabro.

più in buche nella terra e sono abitati da 100 o al più 500 operaie e da una femmina fecondata. Specie comune assai è il $B$. terrestris Ill. di color nero con due fascie, una sul torace, l'altra sull'addome, di color giallo e coll'estremità inferiore dell'addome bianca.

Apis Linn. Le specie di questo genere virono in società e costruiscono nidi assai belli ed ingegnosi. Ciascuna specie comprende tre sorta di individui, vale a dire: maschi o pecchioni, femmine o regine e neutri od operaie. 
La Fig. 160 dà un'idea chiara di queste tre forme.

Le Api costruiscono il nido culla cera (sostanza elaborata dall'Ape stessa). Il nido è formato da una serie di celle esagonali, adossate pel fondo le une alle altre e costituenti un favo. La grandezza delle celle è varia: le più piccole servono a ricevere le larve delle operaie e contengono miele (sostanza zuccherina prodotta dai sughi raccolti dalle api sui fiori e poi elaborati nell'interno dell'organismo) e polline: le altre sono abitate dai maschi e sono piene di solo miele. Qualche cella finalmente è più grande delle altre, ha forma irregolare e serve di aloggio alla larva destinata a divenire regina. Oltre al miele ed alla cera, le Api usano nella costruzione dei favi anche una sostanza resinosa che vanno a raccogliere nelle gemme di varie piante e che vien chiamata propoli. L'accoppiamento si fa volando nell'aria, ed ha luogo una sola volta nella vita della femmina (quattro o cinque anni). Dopo l'accoppiamento la femmina puó a sua voglia produrre maschi o femmine secondo che lascia venire a contatto o no delle uova gli spermatozoi riuniti nella borsa seminale che si trova in comunicazione colla vagina.

Le operaie possono produrre uova da cui si sviluppano maschi. Numerosi sono gli insetti parassiti delle Api. Fra tutti il più dannoso è la Braula coeca (1).

Il genere Melipona Ill. comprende specie affini alle Api, ma più piccole, con zampe più lunghe, con corpo più peloso, che vivono principalmente nell'America meridionale. Il miele da esse prodotto dà luogo ad un importante commercio. $M$. favosa Latr.

(1) Connalia « Della Braula coeca", Giornale l'Apicoltore. Milano, 1870, vol. III. 


\section{Bibliografia.}

Lepeletier de Saint-Fargeav. - Histoire naturelle des Insectes hyménoptères. Paris, 1836-46.

DАнцвом. - Hymenoptera europaea. Lund., 1845.

Gavis. - Ueber die Embryonalhülle der Hymenopteren im Lepidopteren Embryonen. Mém. Acad. de St-Pétersb., vol. XIV, 7ª serie, 1869. - Beiträge zur Kenntniss der Entwickl. bei den Insecten. Zeit.f. Wiss. Zool., 1869.

F. Huber. - Nouvelles observations sur les Abeilles, 2 vol. Paris, 1814.

MATR. - Die Mitteleuropäischen Eichengallen, 1870-71-72, Wienn. - Die europäischen Torymiden biol. und system. bearb. zool. bot. Gesell. Wien, 1874 .

Gravenhorst. - Ichneumologia europaea. Vratislaviae, 1829.

P. Huber. - Recherches sur les moeurs des Fourmis indigènes. Ginevra, 1810. KIRChNER. - Catalogus Hymenopterorum Europae. 1867.

A. Forel. - Les fourmis de la Suisse. Zurigo, 1874.

H. De Saussure. - Études sur la famille des Vespides. Paris, 1852-57.

Sirbold. - Beit. z. Parthenogenesis bei den Artropodon. Leipzig, 1871.

Gerstaecker. - Ueber die geograph. Verbreitung und die Abänderungen der Honigbiene. Postdam, 1862.

A. Dovrs. - Catalogue synonymique des Hyménoptères de France. Amiens, 1874.

S. C. Sxellen vai Wollenhoven. - Pinacographia. Illustrat. of more than 1000 species of North. West. European ichneumonidae sensu linnaeano, 1878.

C. Emerx e A. Forel. - Catalogue des Formicides d'Europe. Mitt. der schweizerischen entomol. Gesell. 1879, vol. V.

C. Emery. - Studi mirmecologici. Bull. Soc. Ent. Ital., vol. II, 1870.-Saggio di un ordinamento naturale dei Mirmicidei. Bull. Soc. Ent. Ital. Anno IX, 1877. 


\section{XXII.}

\section{DISTRIBUZIONE GEOGRAFICA}

Lo studio della distribuzinne gengrafica degli animali, vale a dire lo studio della diffusione delle specie nelle varie località, ha acquistato dopo l'introduzione nella scienza delle teorie Darviniane una grande importanza. Questo studio, tuttavia, specialmente per ciò che è degli insetti, è ben lungi ancora dall'essere compiuto, e le cognizioni che oggi si hanno intorno alla distribuzione geografica di questi animali si riferiscono quasi esclusivamente ai Coleotteri ed ai Lepidotteri, poco o nulla conoscendosi di sicuro intorno agli altri ordini.

La distribuzione geografica degli insetti può, per maggior chiarezza, venir divisa in due, vale a dire:

Distribuzione geografica orizzontale e distribuzione geografica verticale.

La prima studia la distribuzione delle specie nelle varie regioni della terra.

La seconda invece studia la distribuzione delle specie sui. rialzamenti che sorgono alla superficie della terra stessa.

DisTRIBUZIONE GEOGRAFICA ORIZZONTALE.

Gli insetti considerati nel loro complesso obbediscono in generale, rispetto alla distribuzione gengrafica, alle stesse leggi che agiscono sugli altri animali; quindi si possono adottare anche per essi le regole e le leggi stabilite per gli altri.

La terra viene generalmente divisa nelle sei grandi regioni zoologiche (le quali alla lor volta vengono poi suddivise in altre secondarie) seguenti:

$1^{\circ}$ Regione paleartica che comprende tutta l'Europa, incluse le isole Azore, di Madera, le Canarie, del Capor Verde e l'Islanda: la parte settentrionale dell'Af́rica fino al 
tropico del Cancro: l'Asia Minore e quella parte dell'Arabia che è al disopra del tropico del Cancro: la Persia, l'Afganistan ed il Belucistan : e finalmente tutta l'Asia settentrionale e centrale al disopra della catena dell'Imalaia, compresa la penisola di Corea e le isole Giapponesi.

$2^{\circ}$ Regione etiopica che comprende tutta l'Africa centrale e meridionale a partire dal tropico del Cancro: la parte più meridionale dell'Arabia: le isole dell'Ascensione, di S. Elena, di Madagascar, di Borbone, ecc.

$3^{\circ}$ Regione orientale che comprende la maggior parte dell'Asia meridionale, e una parte dell'Asia orientale, e l'arcipelago Malese, vale a dire: la penisola Indostanica e l'isola di Ceylan, l'Indocina colla penisola di Malacca, le isole di Sumatra, di Giava, di Borneo e l'arcipelago delle Filippine.

$4^{\circ}$ Regione australiana che comprende l'Australia propriamente detta, la Tasmania, la Nuova Guinea colle isole di Celebes, Gilolo, Ceram, Salomone, ecc., la Nuova Zelanda e le isole Auckland.

$5^{\circ}$ Regione neartica che comprende l'America settentrionale, gli Stati Uniti: la regione dei Laghi, la Nuova Scozia, la Nuova Olanda, la Groenlandia e le isole Aleutine.

$6^{\circ}$ Regione neotropicale che comprende l'America centrale e l'America meridionale, vale a dire, il Messico, Nicaragua, Costarica, Cuba, Giamaica, Haiti e le Antille; la Colombia, le Venezuela, la Guiana, il Brasile, la Plata, la Patagonia, ecc.; comprende pure le isole Falkland, Giorgio I e Galapagos.

La prima regione o paleartica, può a sua volta, venire divisa nelle seguenti sottodivisioni:

$I^{\circ}$ La prima sottodivisione è formata dall'Europa settentrionale (Islanda, Svezia, Norvegia, Russia), e dall'Europa centrale ed occidentale: ha per confini al Sud i Pirenei, il Mediterraneo, le Alpi, i Balcani, il Mar Nero ed il Caucaso, ad Oriente il Mar Caspio ed il fiume Obi.

$2^{\circ}$ La seconda suddivisione prende il nome di regione Mediterranea e comprende il mezzodi dell'Europa (Spagna, Italia, Grecia), il Nord dell'Africa fino al tropico del Cancro la parte occidentale e meridionale dell'Asia, Asia Minore, Persia, ecc. fino all'Indo.

$3^{\circ}$ La terza sottodivisione è costituita dall'Asia settentrionale 
(Siberia, ecc.), e dall'Asia centrale (Turchestan, Tibet, ecc.).

$4^{\circ} \mathrm{La}$ quarta suddivisione comprende la Cina e le isole del Giappone.

La regione paleartica è relativamente meno ricca d'insettí che non le altre aventi clima più caldo, ed i suoi insetti sono. in generale più piccoli e meno splendidamente coloriti di quelli delle regioni equatoriali. Il clima, tuttavia, assai vario, la svariata natura del suolo delle diverse sottoregioni sopra nominate, fanno si che la fauna della regione paleartica, soprattutto perciò che è degli insetti, sia molto svariata, e porti l'impronta delle faune appartenenti alle regioni limitrofe piư calde. Ciò si osserva soprattutto negli insetti del Nord dell'Africa, i quali ci indicano la vicinanza della fauna africana, e negli insetti della Siberia meridionale e della Cina e del Giappone che offrono parecchie forme schiettamente Indiane.

In quanto ai Lepidotteri osserverò che il numero delle specie di diurni è relativamente alle altre regioni scarso, e non supera molto probabilmente i cinquecento. Molto più numerosi sono i notturni: ma questi sono anche meno conosciuti e molte località della regione paleartica sono per questo rispetto al tutto inesplorate. Dei cinquanta generi circa in cui sono compresi i Lepidotteri diurni della regione Paleartica, quindici circa le sono proprii. Le famiglie dei Lepidotteri diurni che hanno maggior numero di rappresentanti sono le seguenti: Satiridi, Ninfalidi, Licenidi, Pieridi. Fatto caratteristico della fauna della regione paleartica si è la scarsità delle specie di Papilionidi cosi numerosi e cosi splendidi nella regione Indiana e nella regione australiana. E tuttavia si noti, caratteristico. della regione paleartica, l'elegantissimo Parnassius Apollo.

Molto numerose inoltre sono le specie di Zigenidi e di Sfingidi.

La regione paleartica, per ciò che è dei Coleotteri, è caratterizzata dal grande numero di specie di Carabici che essa presenta, e soprattutto per la grande diffusione e pel grande sviluppo del genere Carabus, il quale offre le sue specie piư splendide nella Russia meridionale e nella Siberia.

Poco numerosi sono invece i Coleotteri lamellicorni, rappresentati da circa venticinque generi. Fra questi, ad esempio, i 
generi Trichius, Gnorimus ed Osmoderma sono esclusivi delle parti temperate della regione paleartica.

I Buprestidi hanno il loro massimo sviluppo nella Cina e nel Giappone, ed offrono varie forme analoghe a quelle della regione indiana.

Duecento circa sono i generi di Longicorni riconosciuti esistenti fino ad ora nella regione Paleartica, e di questi una cinquantina circa le sono proprii. Si noti soprattutto fra questi generi il Dorcadion che conta oltre a centosessanta specie, ed il genere Phytoecia che ne comprende una novantina.

Numerosissimi pure sono gli Stafilini, dei quali molti sono i generi speciali alla regione paleartica. Intorno agli insetti di questa numerosissima famiglia non si può tuttavia, fino ad ora, dire nulla di sicuro, essendo a questo riguardo pochissimo note le altre regioni zoologiche.

Fra le altre famiglie di Coleotteri si ricordino i Pimelidi, proprii delle regioni circummediterranee e i Copridi proprii delle regioni meridionali dell'Europa.

Nessun dato sicuro si ha, come ho già avvertito, intorno alla distribuzione geografica degli insetti appartenenti agli altri ordini.

Per ciò che è delle sottoregioni della grande regione paleartica, io non noterò che qua e lá le cose più spiccate, mancando la scienza ancora dei dati necessari per stabilire con esattezza la distribuzione geografica degli insetti dell'Europa stessa, la quale tuttavia è la regione, entomologicamente parlando, la meglio conosciuta. Di qui la necessità delle faune locali coscienziose e diligenti.

L'Islanda, un tempo ricca di foreste e con un clima temperato, ora desolata dal freddo e portante poche Graminacee, che è compresa nella prima sottoregione Paleartica, contiene specie essenzialmente Europee alcune poche solamente appartengono al Labrador ed alla Groenlandia.

La fauna delle isole Inglesi è essenzialmente quella dell' $\mathrm{Eu}-$ ropa centrale e settentrionale; molte specie tuttavia (fatto come vedremo che si verifica spesso nelle isole) sono alquanto modificate (l).

(1) Si consulti, ad esempio, per gli insetti delle isole Shetland, BRown, Trans. of Northern Entomol. Soc., 1862. 
L'Europa centrale, molto ben delimitata al Sud dalle Alpi e dai Balcani, ha un buon numero di specie proprie e la sua fauna per molti caratteri si diversifica da quella dell'Italia e della Spagna. La Francia ha nella sua parte settentrionale e centrale la fauna dell'Europa centrale e nella sua parte meridionale quella delle regioni circummediterranee. Dal punto di vista entomologico, per la sua posizione geografica, per la natura del suolo, ora piano, ora montuoso, ora secco, arido, caldo, ora invece boscoso ed umido, è forse la regione più ricca di tutta Europa. Certamente poi è la regione meglio conosciuta.

Il bacino del Mediterraneo è caratterizzato da una sorta di uniformità molto spiccata delle produzioni vegetali ed animali, uniformità che è in rapporto colla temperatura e col clima che regna eguale in quasi tutti i paesi di questa sotto regione paleartica. La Spagna, l'Italia, la Grecia e la costa dell'Africa offrono, per ciò che è degli insetti, molti punti di contatto.

Nell'Italia noterò che la parte superiore può essere divisa in tre parti: $1^{\circ}$ Piemonte, $2^{\circ}$ Lombardia e Veneto, $3^{\circ}$ Liguria.

Il Piemonte presenta negli insetti, come anche in altri animali, molti caratteri della fauna francese e quindi dovrebbe piuttosto far parte della prima sottoregione paleartica. Un altro argomento in favore di questa riunione stà nell'affinità assai spiccata che vi è fra la fauna entomologica piemontese e la fauna dell'Ungheria. La Lombardia e il Veneto hanno negli insetti un'impronta più meridionale, ed in molti casi fanno il passaggio fra la fauna dell'Europa centrale e quella della regione Mediterranea. La Liguria invece appartiene esclusivamente alla regione mediterranea.

La Sardegna e la Corsica ci offrono campo di verificare molti dei fatti riguardanti le modificazioni che subiscono le specie confinate nelle isole, fatti che vennero principalmente studiati dal Wollaston (1). Molte delle specie di insetti che si trovano sul continente, si trovano pure in Sardegna (la Sardegna è per le ricerche del Genẻ e del Ghiliani relativamente

(1) Wollastow * On the variations of species». London, 1856 . Catalogue on the Coleopterous Insects of Madeira . London, 1857. 
meglio conosciuta della Corsica), ma vi sono in generale alquanto più piccole e leggermente modificate. La Sardegna poi ha molte specie di insetti che si trovano in Sicilia, ed ha anche qualche specie comune colla Spagna e coll'Africa settentrionale. Vari generi le sono proprii.

La Sicilia ha una fauna entomologica che ha molta affinità con quella dell'Italia continentale, della Sardegna e dell'Africa settentrionale.

L'Africa settentrionale ha una fauna che ricorda quella delle coste della Spagna e dell'Italia meridionale, di più ha qualche specie Africana che si spinge fino al Mediterraneo nello stesso modo, come qualche specie mediterranea va fin nel cuore della regione Etiopica.

La terza sotto regione paleartica è poco nota. La Siberia, per quanto se ne sa oggi, è assai ricca di carabici, soprattutto di specie del genere Carabus. I suoi Lepidotteri hanno, in massima parte, il carattere europeo, fatta eccezione tuttavia per quelli del fiume Amur, i quali secondo le ultime ricerche avrebbero giá l'impronta della fauna indiana.

Poco conosciute sono le regioni del Tibet e del Turchestan.

La quarta sotto regione finalmente offre accanto a forme europee forme schiettamente indiane e costituisce un passaggio alla regione orientale.

Riassumendo quindi noi possiamo dire che la regione Paleartica si attacca alla regione neartica per mezzo dell'Islanda, alla regione etiopica per mezzo dell'Africa settentrionale ed alla regione orientale per mezzo della Cina e del Giappone.

La seconda regione, od etiopica, viene a sua volta divisa nelle quattro sottoregioni seguenti :

$1^{\circ}$ La prima sottoregione comprende, a partire dal tropico del cancro, quasi tutta l'Africa, toltone le regioni costituenti l'Africa occidentale (Guinea, ecc.), l'Africa posta al disotto del tropico del capricorno e l'isola di Madagascar. Terreni essenzialmente aridi e sabbiosi (deserti del Sahara e dell'Arabia Meridionale) ed estesi pascoli (pianure di Timbuctu, Kordofan, ecc.) entrano a costituire la prima sotto regione Etiopica. Relativamente scarse sono, per quanto se ne sa oggidi, le foreste in questa estesissima regione. Sono regioni boscose l'Abissinia, le regioni circostanti a Gondohoro e una lunga stri- 
scia di terreno che attraversa longitudinalmente il paese dei Galla e segue la costa orientale dell'Africa fino al fiume Zambesi.

La seconda sottoregione (Africa occidentale) è meno estesa della precedente e comprende le regioni che si estendono dal fiume Gambia fino al fiume Congo e circondano il golfo di Guinea estendendosi nell'interno dell'Africa fin verso il trentesimo grado di longitudine (Greenwich). Questa sottoregione è costituita da terreni coperti da immense foreste e forma la regione più boscosa di tutta l'Africa.

La terza sotto regione comprende l'Africa meridionale dal tropico del capricorno in giù. Fa pure parte di questa regione la costa del Monzambico e del Sofala.

La quarta regione finalmente è costituita dall'isola di Madagascar e dalle isole Borbone, Mauritius, Rodrigues, Seychelles, ecc.

L'Africa, entomologicamente parlando, è poco conosciuta e quindi le nostre cognizioni attuali intorno alla distribuzione gengrafica degli insetti in questa regione non sono molto sicure.

Ottocento circa solamente sono le specie di Lepidotteri fino ad ora state rinvenute in Africa, il che ê molto poco tenendo conto dell'immensità del continente africano. Questo numero sarà tuttavia molto aumentato quando verranno esplorate entomolngicamente le immense foreste dell'Africa occidentale.

Nessuna famiglia di Lepidotteri è esclusiva all'Africa. Vi troviamo tuttavia alcune forme assai caratteristiche soprattutto negli Acreidi, nei Ninfalidi, Licenidi e Papilionidi.

Le forme più strane e caratteristiche sono, come quasi sempre avviene, confinate nelle isole. A tutti è nota la splendidissima Urania rhiphaeus dalle ali dentellate e dai colori di porpora, di smeraldo e d'oro, propria dell'isola di Madagascar.

Fra i Coleotteri abbondano soprattutto i carabici e specialmente la famiglia dei Cicindelidi. Tredici sono i generi di questa famiglia che vi sono rappresentati e di questi undici sono esclusivi della regione etiopica. Basterà ricordare le gigantesche Manticore, le Platichile e le Dromiche ed il genere Pogonostoma proprio dell'isola di Marlagascar. Fra i Carabici citerò i generi caratteristici Anthia, Graphipterus, ecc. 
Una trentina circa sono i generi di Buprestidi Africani, di questi sei sono esclusivi della regione Etiopica. Il più importante fra tutti e il più caratteristico è il genere Polybotris (Madagascar) dal corpo appiattito e dalle elitre foggiate a mo' di paracadute. Si ricordino pure le eleganti specie dei generi Sternocera, Chrysochroa e Julodis. Questi ultimi si incontrano anche nella regione mediterranea.

Fra i Lamellicorni osserviamo pochi Lucanidi e invece molti ed elegantissimi Cetonidi, dei quali sopra settantasei generi sessantaquattro sono caratteristici alla regione etiopica. Menzionerò fra questi soltanto le bellissime e gigantesche specie del genere Goliathus.

L'Africa è meno ricca di Longicorni che non le altre regioni tropicali; duecento sessantadue sono i generi stativi fino ad ora trovati. Questo numero tuttavia aumenterà certamente colle successive esplorazioni del continente Africano.

Considerando ora la regione Etiopica nel suo complesso vediamo che una qualche specie di insetti della regione Mediterranea si avanza anche nel cuore della prima sottoregione (Abissinia): che molte specie sono comuni alla costa occidentale ed alla costa orientale (probabilmente attraversano tutta l'Africa). Che fra la terza sottoregione (Africa meridionale) e l'isola di Madagascar vi sono molte specie comuni (i Lepidotteri di Madagascar hanno pure molta affinità con quelli del Senegal e della Serra Leona) (1), e che finalmente la fauna entomologica dell'isola di Madagascar e delle isole Mauritius e Borbone ha molti punti di contatto con quella delle Indie orientali, dell'Australia ed anche con quella dell'America meridionale.

La terza regione, od orientale, comprende anch'essa quattro sotto regioni. $1^{\circ} \mathrm{La}$ prima sottoregione che vien distinta col nome d'indiana è costituita dalla parte settentrionale e centrale della penisola Indostanica. La parte più meridinnale, l'isola di Ceylan formano la seconda sotto regione (Ceylanica).

La terza sotto regione (Indo Cinese) comprende l'Indocina,

(1) Borspuval " Faune entomologique de Madagascar, Bourbon et Maurice. Lépidot. Parigi, 1833. 
meno la penisola di Malacca, e si estende al nord seguendo le coste del mare fin presso al trentesimo grado di latitudine.

L'isola Formosa fa eziandio parte di questa sotto regione.

La quarta sotto regione (Indo-Malese) finalmente risulta formata dalla penisola di Malacca e dalle isole di Sumatra, di Giava, di Borneo e dall'Arcipelago delle Filippine.

Molto ricca e splendida per bellezza e per stranezza di forme è la fauna entomologica della regione Orientale e molto vi sarebbe da dire intorno alla distribuzione geografica degli insetti in questa regione, la quale contiene molte isole aventi tutte delle speciali produzioni. Io tuttavia non ricorderò che i fatti jiù spiccati e che meglio caratterizzano la regione, rimandando il lettore vago di maggiori particolari alle opere del Wallace (1).

La regione orientale è rispetto ai Lepidotteri caratterizzata dal grande numero e dalla bellezza delle specie del genere Papilio e dalle gigantesche e splendide Ornitoptere. Abbondanti sono pure le specie dei generi Pieris, Terias, Idea, Euplaea, ecc.

Fra i Coleotteri noterò, siccome caratteristici di questa regione, fra le Cicindele i generi Collyris, Dromicidia, Tricondyla e varie specie di Therates. Fra i Carabici poi basterà ricordare gli strani Mormolice e le specie dei generi Thyreopterus, Orthogonius, Catascopus, ecc. Molto numerosi ed eleganti sono i Lamellicorni, soprattutto le specie dei generi Rhomborhina, Macronota, Plectrone, Bombodes, ecc.

Fra i Buprestidi menzionerò le splendide Chrysochroa e le gigantesche Catoxanta. I generi Batocera, Agelasta, Tetraommatus, Rhaphidopodus, ecc., fra i Longicorni caratterizzano pure la regione Orientale. Non si devono dimenticare siccome pure caratteristici di questa regione i generi Chalcosoma, Camposternus, Pachyrhynchus, Diurus, ecc.

La regione orientale si lega per qualche forma alla regione Paleartica, e come abbiamo già detto anche alla regione Etiopica, ma soprattutto poi alla regione Australiana e più precisamente alla sotto regione Austro-Malese.

Quattro sono le sottoregioni che costituiscono la quarta regione od australiana.

(1) Vedi Biblingrafia in fine al capitulo. 
La prima comprende le isole di Celebes, Gilolo, Ceram, Flores, Timor, Nuova Guinea, Salomone, ecc. Tutte queste isole sono coperte in generale di una rigogliosa vegetazione e da folti boschi.

L'Australia propriamente detta, o Nuova Olanda, e la Tasmania formano la seconda sottoregione Australiana. Il paese è poco montuoso ed in generale secco e relativamente povero di foreste e di vegetazione.

La terza sottoregione è costituita da tutte le isole della Polinesia.

La quarta dal gruppo delle isole della Nuova Zelanda.

Quasi tutte le isole che costituiscono la regione Australiana presentano parecchie forme proprie, caratterizzanti la fauna entomologica di ciascuna delle isole stesse.

Considerando la regione complessivamente, possiamo dire che essa non è molto ricca di Lepidotteri, fra i quali spiccano le specie dei generi Papilio, Ornithoptera, Cocytia, ecc.

Molte forme di Lepidotteri, noterò qui di passaggio, sono identiche ad alcune dell'America Meridionale e soprattutto del Brasile.

I Coleotteri sono relativamente più numerosi dei Lepidotteri. Abbiamo parecchie specie di Trycondila, Therates, ecc. Esclusivo della Nuova Olanda è il genere Pamborus che vi tien luogo dei nostri Carabus e delle nostre Calosome.

Molto numerosi invece sono i Lamellicorni ed i Buprestidi. Ricorderó, ad esempio, fra i primi, i generi Schizorhina, Anacamptorhina, Sternoplus e Lomaptera; fra i secondi i generi Stigmodera, Sambus, ecc.

Numerosissimi eziandio sono i Longicorni, di cui la regione Australiana possiede oltre a trecentosessanta generi, dei quali duecento e sessanta circa le sono proprii.

Anche nei Coleotteri come nei Lepidotteri vi ha una spiccata affinità colla regione Neotropicale.

Prima di lasciare la regione australiana dirò che nelle isole Auckland, collocate al sud della Nuova Zelanda, ricompaiono in parte nei Carabici le forme del Nord d'Europa, e vediamo ad esempio ai nostri antipodi ricomparire i generi, Calathus ed Argutor.

La quinta regione, o neartica, contiene le seguenti sotto- 
regioni : $1^{\circ}$ La prima sottoregione comprende una stretta striscia di paese sulla costa occidentale dell'America settentrionale, dal trentesimo al quarantesimo grado di latitudine. La seconda sottoregione è costituita essenzialmente dalle Montagne rocciose, dalla penisola Californica e da una parte del Messico settentrionale e giunge fin verso il $100^{\circ}$ di longitudine (Greenwich). La rimanente parte degli Stati Uniti, fino verso il cinquantesimo grado di latitudine, forma la terza sottoregione. La quarta finalmente è costituita dalle parti più settentrionali dell'America a partire dal $50^{\circ}$ di latitudine.

La fauna entomologica della regione neartica ci presenta una grandissima affinità con quella della regione paleartica ; molti generi sono comuni alle due regioni, e varie specie anzi sono al tutto identiche. Nello stesso modo tuttavia in cui la regione paleartica sente l'influenza della regione orientale ed un po' anche della regione etiopica, la regione neartica mostra in molte forme l'impronta della fauna neotropicale.

La regione neartica è molto ricca di Lepidotteri e soprattutto di Sphinx e di Papilio.

Fra i Coleotteri poi noterò il genere Scaphinotus e Sphaerotus che vi rappresentano i nostri Cychrus, i generi Pasimachus, Dicaelus, Phanaeus, ecc.

Neotropicale ha nome la sesta regione, la quale, come le altre, può a sua volta venir divisa in quattro sottoregioni. La prima sottoregione comprende nell'America meridionale la costa occidentale fin verso l'equatore, la Patagonia, la Plata, la Bolivia ed il Perù. Questa regione è in parte montagnosa, in parte al tutto piana, non è molto ricca di foreste, ma ha invece immense praterie o Pampas.

Il Brasile propriamente detto, le Guiane, la Venezuela e la Colombia costituiscono la seconda sottu regione. La terza comprende l'America centrale, il Messico, l'Honduras e Costa Ricca. La quarta finalmente è formata dalle isole delle Antille.

La regione Neotropicale è la più ricca di tutte in insetti ed è anche quella che racchiude le forme più belle e più splendide. Gli insetti non sono tuttavia sparsi egualmente in tutta la regione. Abbondano principalmente nella seconda sottoregione, ricca di acque e di rigogliosa vegetazione e soprattutto nella valle delle Amazzoni, che è la località del mondo più ricca 
di tutte di insetti. Scarseggiano a misura che si discende verso la prima sottoregione.

Fra i Lepidotteri menzionerò le fragili e trasparenti Itomie rappresentate da oltre 160 specie; i bellissimi Satyrus, i giganteschi Morpho dai colori metallici iridescenti e darli splendori madreperlacei, le elegantissime e variopinte specie dei generi Catagramma e Callithea; le numerosissime specie di Erycinidae e di Hesperidae e le grandi e bellissime Urania.

Oltre ogni dire abbondanti sono poi i Coleotteri e numerosissimi pure sono i generi proprii esclusivamente di questa regione.

Fra i Cicindelidi noto i generi Oxychila, Hiresia, Ctenostoma ed Odontochila (quest'ultimo genere conta oltre a cinquanta specie ed il genere Tetracha che noi abbiamo già incontrato nelle regioni Paleartica, Australiana e Neartica. Fra i Carabici sono da ricordarsi soprattutto i generi seguenti caratteristici della regione Neotropicale: Agra, Ardistonus, Schizogenius, Antarctia, Tropidopterus, ecc.

Molte pure sono le specie di Lucanidi dell'America meridionale e deve essere notato soprattutto lo stranissimo genere Chiasognathus proprio del Chili.

Mediocremente numerosi sono invece i Cetonidi ed i Buprestidi. Fra i primi trovasi da notare il genere Inca: fra i secondi molti generi sono comuni all'America settentrionale, all'Australia ed all'isola di Madagascar.

Numerosissimi invece e molto belli sono i Longicorni; vi si incontrano 520 generi circa e di questi 490 circa sono propri esclusivamente della regione Neotropicale. Abbondano soprattutto i Prionidi coi bellissimi generi Psalidognathus, Pyrodes e Macrodontia. Non meno numerosi sono pure i Cerambicidi cogli eleganti e variopinti Ibidion e Chrysoprasis ed i Lamidi cogli strani Macropus, cogli Hemilophus, ecc.

Si è pure nella regione Neotropicale che troviamo fra i Corculionidi i bellissimi Entymus, Cyphus, Diapiris, ecc.

I Crisomelini poi prendono nell'America meridionale un enorme sviluppo e ci si presentano sotto forme gigantesche, elegantissime e strane. Ricorderò le Dorifore, le Calligrafe e soprattutto i gibbosi Erotili quasi al tutto esclusivi all'America meridionale. 


\section{DISTRIBUZIONE GEOGRAFICA VERTICALE.}

Dato cosi uno sguardo generale e rapidissimo alla distribuzione orizzontale degli insetti alla superficie della terra, vediamo brevemente come siano distribuiti gli insetti sulle protuberanze della terra stessa.

Studiando la fauna delle montagne noi vediamo subito che esse non sono favorevoli allo estendersi dei viventi e che quanto più sono elevate tanto minore è il numero degli animali che esse racchiudono e finalmente che il numero delle specie e degli individui diminuisce a misura che noi dalla base ci portiamo verso la cima.

Le montagne, per ciò che riguarda la fauna, vennero divise in varie zone. La prima va fino ai 1500 metri e contiene ancora molte delle forme del piano. La seconda dai 1500 sale fino ai 2500 e comprende essenzialmente la regione delle conifere ed ha un grande numero di forme esclusive delle montagne. L'ultima finalmente parte dai 2500 metri e va fino alle sommità più elevate. Quest'ultima zona è priva, in generale, di vegetazione arborea ed è povera di animali. Le poche forme tuttavia che essa racchiude sono caratteristiche di questa regione e sono interessantissime.

La fauna entomologica varia a misura che ci innalziamo sul livello del mare nello stesso modo in cui varia la fauna entomologica del piano a misura che dall'equatore ci portiamo verso il polo.

Di tutti gli insetti i Ditteri (Chironomus) sono quelli che si spingono più in alto sui monti, e sono pure quelli che si trovano a latitudini più elevate. Ai Ditteri tengono dietro i Coleotteri, poi vengono gli Imenotteri, i Lepidotteri, gli Ortotteri, gli Emitteri ed i Nevrotteri (1).

(1) Varie specie di insetti, soprattutto Lepidotteri e Coleotteri, presentano a grandi altezze i loro colori più o meno modificati; per lo più le tinte si inscuriscono. Non si sa ancora dare una spiegazione soddisfacente di questo fatto. Lo stesso fenomeno del resto si osserva pure nelle specie che si spingono molto al Nord dell'Europa. Un'altra modificazione che si incontra assai frequentemente nelle specie di Coleotteri che vivono a grandi altezze si è l'atrofizzarsi delle ali membranose. Questo ridursi delle ali nei Coleotteri delle alte regioni delle montagne mi pare si possa spiegare nello stesso modo in cui il Wollaston spiegò l'atrofizzarsi delle ali nei Coleotteri dell'isola di Madera. 
Andando dalla base delle montagne verso la sommità, noi vediamo diminuire il numero delle specie fitofaghe e crescere invece relativamente quello delle specie carnivore e ciò come ognuno facilmente intende è in rapporto col modificarsi della vegetazione. I Carabici fra i Coleotteri sono probabilmente quelli che salgono più in alto. Alle leggi ora enunciate si incontrano tuttavia molto frequentemente eccezioni più o meno spiccate. Così ad esempio non è cosa rara il trovare nelle Alpi delle regioni ristrette od oasi aventi una flora od una fauna affatto diversa dalla flora e dalla fauna circostante e che ha molta analogia invece con regioni lontane. Per non citare che località a noi vicine, menzionerò la valle di Susa, la quale ha qua e là delle regioni che presentano piante, insetti e molluschi proprii della Liguria e delle spiaggie circummediterranee. Qualche volta finalmente certe regioni del piano (come è il caso per qualche tratto della collina di Torino) presentano, sebbene in un grado non molto spiccato, l'impronta della fauna alpina.

Esaminata cosi per sommi capi la distribuzione geografica degli insetti, vediamo quali siano le leggi che, per quanto ne sappiamo oggi, pare presiedano ad essa. Menzionerò le priıcipali :

$1^{\circ}$ Le variazioni della fauna sono piu spiccate in generale seguendo i gradi di longitudine che non seguendo i gradi di latitudine.

$2^{\circ} \mathrm{A}$ misura che dall'equatore si va ai poli le faune delle varie regioni zoologiche assumono un'impronta comune (1).

$3^{\circ}$ La maggiore divergenza delle faune si osserva nelle latitudini prossime all'equatore.

$4^{\circ}$ Hanno grandissima importanza nella distribuzione geografica degli insetti le linee isotermiche.

$5^{\circ}$ La fauna delle alte regioni ha in quasi tutte le regioni zoologiche un'impronta comune. Questa legge è in stretto rapporto colla seconda.

$6^{\circ} \mathrm{Gli}$ insetti acquaiuoli sono fra tutti quelli che variano meno nelle diverse regioni.

(1) Abbiamo visto, ad esempio, che la Nuova Zelanda e le isole Auckland presentano molte forme affinissime a quelle dell'Europa settentrionale. 
$7^{\circ}$ La natura del suolo, il grado di umidità, la vegetazione, la temperatura, ecc. sono gli agenti che più efficacemente modificano la fauna di una data località.

$8^{\circ}$ Fra la fauna entomologica di due località appartenenti a due regioni zoologiche diverse, ma aventi eguale clima ed eguali condizioni di temperatura e di suolo, esiste quasi sempre molta affinità.

$9^{\circ}$ Le isole, anche vicine le une alle altre, offrono quasi sempre una grande diversità nelle loro faune.

10. Nello studio della distribuzione geografica degli insetti ed in generale di tutti gli animali è cosa molto importante il tener conto dei dati che la geologia ci porge intorno al modo di formazione delle varie regioni.

11. Non si debbono pure dimenticare tutte quelle cause spesso accidentali che vengono a modificare la distribuzione degli insetti di una data località come i venti, i fiumi (1), le montagne, l'azione dell'uomo, ecc.

12. Dobbiamo finalmente tener conto delle migrazioni, le quali avvengono negli insetti piu frequentemente forse di quello che non si creda ordinariamente (2).

(1) I fiumi quando ingrossano trasportano coi detriti vegetali un grande numero di insetti che poscia depongono a mano mano sulle rive. L'uomo, per mezzo dei bastimenti, ha favorito la diffusione per tutta la terra di molte specie di insetti, ad esempio le Blatte fra gli Ortotteri, i Dermesti e gli Antreni fra i Coleotteri.

(2) Oltre alle emigrazioni, note a tutti, delle Cavallette e di altri Ortotteri, sono stati osservati altri casi di insetti appartenenti ad altri ordini. Per non uscire dal Piemonte menzionerò l'emigrazione osservatavi nel 1867 e negli anni consecutivi fino al 1873 di un grandissimo numero di Anax mediterraneus $D e$ Selys (specie che come fece notare il Ghiliani « Acclimazione spontanea », Bull. Entomol. Ital., anno I, finì per acclimarsi in Piemonte). Nel 1851 come riferisce lo stesso Ghiliani (1): «Videsi un passaggio straordinario di Vanessa cardui Linn. diretto dal sud-sud-est al nord-nord-ovest, e, caso singolare, viaggiante quasi a contro vento». Lo stesso fatto si verificò pure in questo stesso anno (1879). Vedi Cameraxo, Rendiconti della Soc. Ent. Ital., 1879.

(1) Migrazione d'insetti, Gazzelta di Torino, 1867, $\mathrm{n}^{\circ}$ 272-277-250. 


\section{Bibliografia.}

A. R. Wallace. - The Geographical distribution of Animals. Two. vol. London 1876.

- The Malay Archipelago. London, 1869.

F. Tscnudr. - Die Alpenwelt, tradotto in francese « Les Alpes ».

G. Косн. - Die indo-australiche Lepidopterenfauna in ihrem Zusammenhauge mit den drei Hauptfaunen der Erde. Berlin, 1873.

Azambre. - Note sur la géographie entomologique de quelques Lépidoptères. Ann. Soc. Ent. Franc., 3 a serie, 1856, vol. 4.

Blanchard. - De la distribution géographique des animaux articulés. Revue zool., 1841.

A. Costa. - Rapporti di geografia entomologica tra il Regno di Napoli e la Sardegna. Rend. Acad. d. Sc. di Napoli, 1853.

Ghiliani. - Note sur certain Lépidoptères de l'Andalousie trouvès en Piemont. Ann. Soc. Ent. Franc., 1851.

- Mémoire sur la station de quelque Coléoptères dans les différentes régions du Piemont. Ann. Soc. Ent. Franc., 1847.

L. Schmarda. - Die geographische Verbreitung der Thiere. Wien, 1853.

ERIchson. - Beitrag zur Fauna von Vandiemensland, ecc. Wiegmann Archiv., 1842.

- Beitrag zur Insecten-Fauna von Angola in besonderer Beziehung zur geographischen Verbreitung der Insecten in Afrika. Wiegmann Archiv., 1843. 


\section{INDICE DEI GENERI}

\section{A}

Acalles 68

Acanthia 50, 52, 236, 237, 219.

Acanthopus 51

Acherontia $68,82,27 \%$

Acheta 149.

Achias 117

Achorutes 172.

Acidalia 267

Acilius 302

Acrididi, 174.

Acridium 171, 186, 187, 189.

Acrocinus 51, 90.

Aeschna 139, 171 .

A fide 41 .

Agathidium 58.

A yrion 171.

A yrotis 66, 263, 269.

A kis 53 .

Aleurodes 137, 227.

Alucita 263.

Ameles 182

A metamorphota 161 .

Andrena 124.

Aneloptera 164

Anfibiotici 195.

Anisoxelis 238

Anisomorpha 53,181

A nobium 68, 296.

Anonconotus 192.

A nophtalmus 94, 303.

Anoplura 164

Anostostoma 50, 169, 192.

Anthia 50 .

Anthicus 295.

Anthocharis 68 .

Anthrenus 300.

Apachya 176

A phaniptera 164.

A phis 54, 129, 227, 228.

A phodius 298

A phrophora 232.

A pion 293.

A pis $4,5,23,16,41,47,49$, 320 .

Aptera 164

Aptinus 52

Architteri 41

Argynnis 280

Arumia 53, 291.

Ascalaphus 214

Asida 53

Asilus 252.

Aspediotus 137.

Ateuchus 298 .

Atta 316 .

Atteri 225.

Autliata 164

\section{B}

Bacillus 45, 51, 135, 170, 171 184.

Bacteria 171, 181

Balaninus 293.

Batrachomya 249

Belostoma 23.1.

Bembidium 303

Berosus 101

Bibio 254

Bittacus 207, 211, 209

Blabera 171, 180.

Blaps 52, 29.1, 29.5.

Blatta 171, 177, 179, 191

Blepharis 51, 183 .

Bombus 50, 56, 78, 321.

Bombylius 252

Bombyx 76, 81, 108, 272.

Boreus 207, 209, 211.

Bostrychus 29:.

Botys 266.

Brachicerus 53

Brachinus 52, 131, 303.

Bracon 29, 313.

Brachylabis 176

Braula 94, 218.

Brenthus 294.

Broschus 76, 80 .

Bruchus 294.

Buprestis 297.

\section{C}

Calandra 15, 293

Callidium 291.

Callimorpha 270.

Callineinus 192.

Calliphora 37

Caloptenus 187, 189

Calopteryx 171, 203.

Calotermes 171.

Calosoma 50,52, 304.

Campodea 87, 171.

Campoplex 29.

Capnodis 53 .

Carabus 50, 52, 148, 303.

Carpocapsa 265.

Cassida 15, 1.1, 51, 68, 289

Catocala 82, 208. 269.

Cebrio 17

Cecidomyia 256.

Centrotus 232

Cephalocteus 94

Cephalomia ovis 250 .

Cephus 312.

Cerambyx 291

Cerapholitha 265

Ceratocampa 51.
Cerceris 318

Cetonia 53, 80, 300.

Chalcosoma 50

( haudiodes 210.

Chelidura 174, 177.

Chiasognathus 50, 298

Chironomus 137,255 .

Chlaenius 52, 96 .

Chorista 211

Chrysis 54, 317.

Chrysochroa 14.

Chrysomela 290.

Chrysopa $53,212$.

Cicada 67, 233.

Cicindela 50, 51, 51, 79, 124 , 301.

Cimbex 121, 311

Cimex 221.

Cis 295 .

Clambus 58

Cleonus 53

Clivina 54

Clytus 291.

- Cnethocampa 53, 271, 272.

Coccinella 52, 289.

Coccus 83, 126, 226

Cochlophanes 274 .

Cochylis 265 .

Colaspis 15 .

Collyris 57.

Colly rodes $5 i$

Coniopteryx 213.

Conocephalus 189

Conops 219

Copris 53, 65, 67, 29s.

Coptoteryx 181.

Cordulia 171, 204

Corethra 255

Coreus 238.

Corixa 231

Corydia 179

Corydalis 50, 206, 209, 210 .

Cossus 16, 991, 275, 116.

Crabro 318

Cryptocercus 180

Cryptus 29.

Culex 50, 255.

Cybi-ter' 302 .

Cychrus 67, 94, 301 .

Cylindrogaster 174, 176.

Cynips 312 .

Cyphocrana 181

Cyphon 80.

\section{D}

Decticus 171, 190, 191.

Degeeria 171, 173.

Dermestes 300 . 
Dicerca 53, 58, 96, 298 .

Dinastes 46.

Diopsis 117, 248.

Dolichus 80 .

Doliops 57.

Dorcus 50, 64.

Doria 29.

Doriphora 289

Dorthesia 227.

Drilus 63, 296.

Dytiscus 52, 64, 71, 30\%.

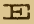

Echinomyia 149.

Echinosoma 176

Ectobia 49, 177, 178

Edessa 238

Ephemera 139, 171, 199, 200.

Ephippigera 171, 192.

Elater 127, 297.

Embia 171.

Emesa 236.

Empis 251.

Empusa 51, 171, 183

Emus 56.

Entimus 15, 80, 293.

Epacromia 188.

Epomis 49.

Erebia 279.

Eremiaphila 18\%.

Euprepia 270, 271.

Euryacantha 181

Evania 314.

Exorista 29.

\section{E}

Fischeria 183 .

Forficula $36,112,171,175$.

Formica 4, 16, 41, 194. 316.

Fulgora, 66, 232.

\section{$G$}

Gastropacha 273, 271.

Gastrus 250.

Geniates 61, 79, 80.

Geocoris 237.

Geotrupes 298

Gerris 83, 101

Glossinia 50 .

Gomphocercus 188

Gomphus 171, 203.

Gryllotalpa 56, 171, 193

Gryllus 171, 193, 195

Gynandromorphus 49

Gyrinus 52, 79, 117, 303

\section{EI}

Haematobia 50.

Halobates 101, 235.

Haltica 290.

Haplogenius 214.

Harpajus 51, 5t, 76, 303.

Hedychrum 58

Helicopsiche 208, 215

Heliothrips 195.

Helophorins 101.

Hemerobius 212.

Hemesodromia 251.

Henops 252.
Hesperia 278.

Heterogamia 171, 177

Heterorynis 30,63 .

Heterotuma 96.

Hibernia 267.

Hippobosca 137, 248.

Hispa 289

Hister 95, 301

Homoeoyamia 180.

Hoplia 14, 80, 299.

Hybos 252

liydrobia $6 \pi$.

Hydrobius 145 .

Hydrocampa 267 .

liydrometra 7.1, 235.

Hydrophilus $38,64,67,69$, $79,156,302$.

Hydrophorus 302.

Hylesious 292.

Hylobius 293.

Hylotoma 311 .

Hypocephalus 56 .

Hypoderma 250.

Hypocyptus 58 .

\section{丁}

Jaera 35

Japyx $38,86$.

Jassus 83,231 .

I

Kallima 55

Labia 176

Labidura 171, 176.

Lachnus $2: 8$.

Lamia 291.

Lamprima 50.

Lampyris 66, 296.

Lasia 252.

Lecanium 83, 137, 226.

Ledra 231.

Lepisma 81, 171, 173.

Leptalis 57 .

Leptis 253.

Leptura 290

Libellula $83,171,20-1$

Limnophilus 216.

Liparis 84, 270, 271, 272.

lithosia 53, 270.

Lipura 171.

Locusta 15, 41, 124, 171, 191

Lucanus 15, 45, 50, 64, 298.

Lucilia 249.

Lycaena 278.

Lygaeus 237.

Lystra 83, 233.

Lytta 52, 294.

\section{M}

Machilis 171, 173

Macrodontia 15, 50

Macroglossa 276.

Mamestra 66, 269.

Manticora 305.

Mantis 51, 168, 170, 171, 174,182

Mantispa 51, 207, 209, 211.
Masicera 29

Mecitogaster 203.

Meconema 171, 190.

Megacephala 50.

Meraceras 65.

Megralopus 52.

Meyasoma 65.

Melanothrips 195

Melipona 322

Meloè 160, 294.

Melolontha 80, 146, 299.

Melophagus 218.

Mesochorus 29

Metalleutica 182.

Metamorphota 164

Metrocaupa 68.

Miastor 151, 256.

Microlepidotteri 263.

Miris 137.

Mogoplistes 193

Molorchus 56.

Monophlebus 226.

Mordella 116.

Mormolyce 301 .

Morpho 15.

Musca 105, 111, 158, 249

Mylabris 52, $160,295$.

Myrınecophila 171, 193 .

Myrmeleo 213.

Myrmica 50.

Myschoris 219

Myxzoxylus 83 .

\section{IV}

Naucoris 149

Necrophorus $53,67,301$.

Neides 238.

Nemoceri 254.

Nemoptera 104, 207, 213

Neinura 171, 200.

Nepa 51, 54, 137, 234, 235.

Neurotteri 6, 41 .

Neusonia 216.

Nicoletia 171, 173.

Nitidula 301.

Notonecta 4i, 50, 83, 234.

Nottuiformi 255 .

Nottuini 268

Nycteribia 94, 137, 218.

Nymphula 267.

Ocypus 96.

Odonata 164

Odontura 192.

Oecanthus 194

Dedemera 291.

Oedipoda 171, 8\%, 189.

Oestrus 95.

Omophron 54, 394.

Onconotus 192.

Onthophagus 53, 118, 298.

Opatruin 53.

Ophion 53, 314.

Opbonus 49.

Orryia 272.

Ornithomyia 137, 218.

Orvithoptera 282.

Oryctes $15,65,127,130,300$

Osmylis 212.

Otiorynchus 53, 58, 292.

Oxaena 52

Oxycheila $6 \%$. 


\section{P}

Pachycoris 238.

Pachygaster 253

Pachyrhynchus $15,57$.

Pachytylus 187, 189.

Palinyenia 171.

Panagaeus 52.

Panorpa 209, 211.

Papilio 68, 281.

Parassita 164.

Passandra 50.

Paussus 52, 118.

Pediculus 225.

Pentatoma 54, 150, 238, 239.

Periplaneta 49, 53, 171, 178, 179.

Perla 171, 200.

Perilitus 29.

Pezomachus 29.

Phanaeus 53.

Phaneroptera 190.

Phasma 44, 135, 170, 171, 184.

Pholidotus 50.

Phora 248.

Phoraspis 177.

Phosphaenus 65.

Photophorus 65 .

Phryganea 216.

Phthirius 225.

Phyllium 44, 51, 171, 183, 185.

Phyllodromia 177.

Phyllomorpha 238.

Phylloptera 190.

Phylloxera 153, 228.

Phyrophorus 297.

Pieris $41,281$.

Piezata 164

Pimelia 53, 295.

Pimpla 29, 315.

Piophila 248.

Platicerus 50.

Platycnemis 201.

Platypeza 251.

Plea 234.

Plusia 268

Pneumora 174, 185, 187.

Podura 31, 171, 172, 173

Polynema 101.

Polyommatus 278 .

Polyphaga 179

Polyphylla 80,300 .

Porphyrophora 227

Polysosteria 177, 179.

Praepodes 15.

Prionus 15

Prionotus 5\%.

Prisopus 171, 184

Procrustes 121

Proscopia 171, 189.

Psalicerus 50.

Pselaphus 116, 301.

Psocus 171, 196.

Psylla 231.

Pterocroza 190.

Pteronarcys 140, 198, 200.

Pteromalus 29, 313.
Pterophorus 263.

Pterostichus 53.

Pulex 147, 256.

Pycnogaster 192.

Pyralis 266.

Pyrrhocoris 237.

I

Ranatra 51, 54, 137, 235.

Raphidia 210.

Rauhidophora 192.

Raphigaster 52.

Reduvius 50, 236.

$\mathrm{R}$ haphiderus 184 .

Rhipiphorus 295.

Rhisotrogus 36,300 .

Rhipiptera 164.

Rhynchites 293.

Ringia 251.

Rogas 29.

Rosalia 291.

S

Saga 171,192

Saperda $290,291$.

Sarcophaga 137, 219.

Sarcopsylla 256.

Sargus 253.

Satirus 147, 279.

Saturnia 273, 275 .

Scarabaeus $3,15,50,53$

Scarites 303 .

Scatophaga 248.

S hizocephala 171 .

Sciara 255.

Scolia 317.

Scotodipnus 167

Scutellera 239.

Sericaria 31.

Sericostoma 215.

Sesia 56, 81, 276.

Sphaeromorphus 58 .

Sphinx 53, 54, 65, 66, 277.

Sphodrus 89,90 .

Sialis $140,209,210$

Silpha 301 .

Simulia 254 .

Simulium 50 .

Sirex 311.

Sisyra 212.

Sitaris $160,295$.

Sitotroga 53 .

Sinerinthus 278.

Smicra 92

Smynthurus 171, 173

Staphylinus 124,302 .

Stauronotus 187 .

Stenobothrus 188.

Stetheophyma 188.

Stilbum 54, 58.

Stratioin ys $75,253$.

Strepsitteri 217.

Stomis 76.

Stomoxys 50

Stylops 95, 217.
Suctoria 164

Synamostes 58 .

Synistata 164.

Syntomis 275 .

Syrphus 251 .

Syrtis 237.

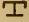

Tabanus 253

Tachina 29, 124, 249.

Teleas 29

Telephorus 296.

Tenebrio 294, 295.

Tenthredo 311

Termes 16, 171, 197, 198.

Tesseratoma 219.

Tétraops 117.

Tetraptera 164.

Tettigonia 16, 124, 231 .

Tettix 171, 185, 187.

Tetyra 238 .

Thecla 278 .

Theoclytes 183 .

Thereva 252.

Thrips 171, 195.

Thyreophora 66 .

Timarcha 52.

Tinea 264.

Tinyis 237 .

Tipula 137, 256.

Tortrix 26.

Trachys 298.

Trichodectes 225 .

Trichodes 296.

Tridactylus 193.

Troctes 171, 196.

Trox 67.

Truxalis 168, 171, 189.

Trypeta 248.

Typhlopona 94 .

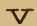

Vanessa 68, 82, 138, 279, 280.

Vespa 50, 319 .

Volucella 56,251 .

\section{X}

$\mathrm{Xenos} 95,116,217$.

Xilophagus 253.

Xiphidium 190 .

$\mathrm{X}$ ylocopa 321 .

\section{Y}

Yponomeuta 263.

Zerene 268.

Zoolea 183.

Zygaena 5\%, 275, 276. 


\section{INDICE DEI CAPITOLI}

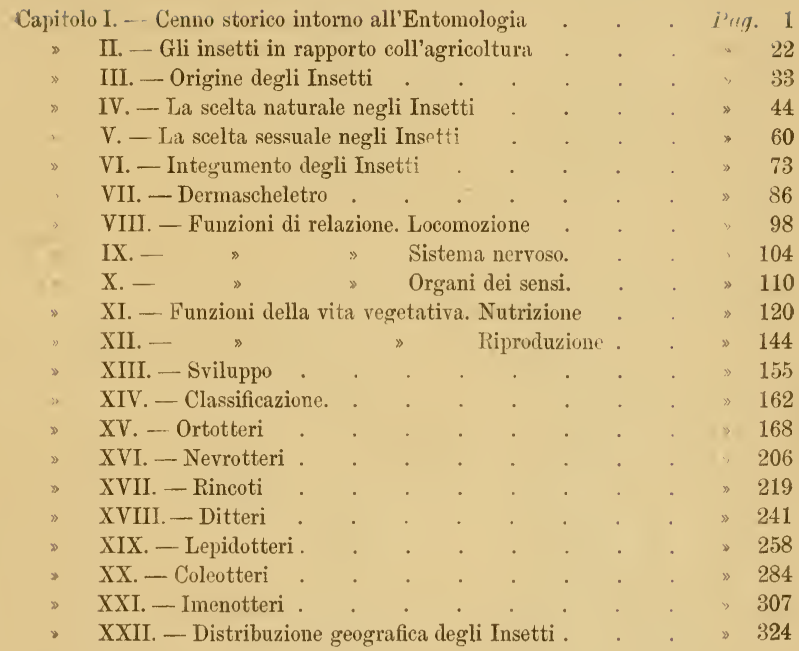




\section{ERRATA-CORRIGE.}

Invece di

Pag. 5, linea 2 Leuwenhoeck

\section{Leggasi}

Leeuwenhoeck (Questa correzione deve farsi pure in vari altri luoghi).

Déjean (Questa correzione deve farsi pure in vari altri luoghi).

" 11 \$ 36 larves . . . . . . larvae

„18, 19 Audonin. . . . . Audouin

$\gg 32 \gg 1$ insettivi . . . . . insettivori

„ 79 » 25 millimetri . . . millimetro

Fig. 22 . . . $g$. . . . $y$

๖. . . a . . . $x$

》. . . . $x$. . . . . $a$

Pag. 123 linea 33 Ditischi . . . . Ditisci

* 128 * 26 e la maggior dei Coleotteri . . e la maggiorparte dei Coleotteri

* 140 » 40 Pteronacris . . . Pleronarcys

* 150 » 32 Leyolig . . . . Leydig

" 154 > 18 parte . . . ponte

> 156 * 25 nella maggior parte di nella maggior parte dei casi di

» 182 » 14 colore terreno . . colore del terreno

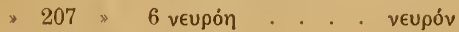

, 250 » 13 Hercolani. . . Ercolani

* 254 » 24 Brachieri. . . Brachiceri

Fig. 123 Hylobius pini . . . . . Rhynchites betuleti

» 124 Rhynchites betuleti . . . Hylotrus pini

Alcuni altri errori incorsi nella stampa di quest'opera possono essere agevulmente corretti dal lettore stesso. 



I 
